Violent Extremist OrganizationsAuthor(s): Brigit Davis

Source: Journal of Strategic Security, Vol. 12, No. 3 (2019), pp. 37-156

Published by: University of South Florida Board of Trustees

Stable URL: https://www.jstor.org/stable/10.2307/26775814

\title{
REFERENCES
}

Linked references are avalable on TSTOR for this article:

https://ww jstor org/stable/10.2397/26775814?seq=1\&cid=pdf-

reference\#references_tab_contents

You may need to $\log$ in to J'STOR to access the linked references.

JSTOR is a not-for-profit service that helps scholars, researchers, and students discover, use, and build upon a wide range of content in a trusted digital archive. We use information technology and tools to increase productivity and facilitate new forms of scholarship. For more information about JSTOR, please contact support@jstor.org.

Your use of the JSTOR archive indicates your acceptance of the Terms \& Conditions of Use, available at https://about.jstor.org/terms 


\section{Violent Extremist Organizations: Past Trends and Short-Term Forecast}

Brigit Davis

Henley Putnam School of Strategic Security, brigitdavis3@gmail.com

Follow this and additional works at: https://scholarcommons.usf.edu/jss pp. 37-156

Recommended Citation

Davis, Brigit. "Violent Extremist Organizations: Past Trends and Short-Term Forecast." Journal of Strategic Security 12, no. 3 (2019): : 37-156.

DOI: https://doi.org/10.5038/1944-0472.12.3.1737

Available at: https://scholarcommons.usf.edu/jss/vol12/iss3/3

This Article is brought to you for free and open access by the Journals at Scholar Commons. It has been accepted for inclusion in Journal of Strategic Security by an authorized editor of Scholar Commons. For more information, please contact scholarcommons@usf.edu. 


\title{
Violent Extremist Organizations: Past Trends and Short-Term Forecast
}

\section{Author Biography}

Brigit Davis is a consultant for a global technology and consulting company with 17 years of experience in the Intelligence field. She served in the United States Air Force from 2001-2009 and has a Bachelor of Arts degree in Intelligence Studies and a Master of Arts degree in Intelligence Operations from American Military University. She is currently a doctoral student at the Henley Putnam School of Strategic Security, National American University.

\begin{abstract}
Violent extremist organizations (VEOs) are the focus of an extensive amount of research by academic and government entities, some of whom have devised complex forecasting methodologies. This study takes a step back from these methodologies and provides one that uses simple mathematical computations (that is, percentages and modes) while still providing slightly more granularity than currently available forecasts. By using data from the University of Maryland Global Terrorism Database and the Terrorism Research and Analysis Consortium, this study identifies trends related to the number of emerging VEOs, ideology types, locations of emergence/operation, attack types, target types, and disbandment. These trends have remained relatively consistent for 25 years and are the basis for a forecast that looks at what the global VEO threat is likely to look like in the next 5 to 10 years. Of fundamental interest are 18 countries that have a higher probability of multiple VEOs emerging within a single year: Afghanistan, Bangladesh, the Central African Republic, the Democratic Republic of the Congo, Egypt, India, Iraq, Israel, Mali, Niger, Nigeria, Pakistan, Palestine, Philippines, Russia, Somalia, Sudan, and Syria. This study also engages in a qualitative discussion on what these trends may mean for the United States, and tangentially the international community, as VEOs continue to pose a significant threat locally, regionally, and nationally.
\end{abstract}

\section{Acknowledgements}

I want to thank Robert Rehbein for his support and encouragement throughout this process. Without his help, this study would not be what it is today.

This article is available in Journal of Strategic Security: https://scholarcommons.usf.edu/jss/ vol12/iss $3 / 3$ 


\section{Introduction}

Violent extremist organizations (VEOs) are a continuing threat to the United States and international community and have, therefore, become the focus of an extensive amount of research by academic and government entities. Research has typically focused on historical and current VEOs in the hopes of generating an understanding of why VEOs emerge; where they operate; the attack methods they use; the targets they attack; and why they may disband. Even though continuing this type of research is imperative in understanding VEOs at a tactical, operational, and strategic level, an equally comprehensive research effort is necessary to forecast the VEO threat at a strategic level. Unfortunately, available forecasting research appears to be either preoccupied at the tactical level or more concerned with discussing how or whether we should be engaging in such endeavors than on providing actual forecasts.

In the studies reviewed, all researchers agree that existing methodologies to forecast the VEO threat are mostly unsuccessful. ${ }^{i}$ Despite this train of thought, studies continue to employ variations on the same method of using qualitative trend analysis (in other words, societal or behavioral trends) in combination with available technologies to obtain a quantitative result.ii The complexities involved in quantifying qualitative data results inevitably in unreliable forecasting that makes it challenging for researchers to inspire confidence in the results by government entities. This lack of confidence then transfers over to available forecasts in the form of generic statements such as: "disaffected migrants without better integration, education and economic opportunity could become an ideal recruiting pool for VEOs," one of many such generic statements in a study published by the Office of the Director of National Intelligence. iii Although accurate, this generic forecast statement applies to any country that contains migrants and therefore does not provide any useful insight for posturing and planning against the future VEO threat at a strategic level.

This study seeks to take a different approach in forecasting by looking at fact-based data that is easily quantifiable as the most straightforward approach is often the best one. The premise is that by going back to fundamental statistical analysis, this study can provide a strategic level forecast that is specific yet broad enough to aid in developing focused and practical strategies to identify and mitigate the VEO threat in the nearterm. Based on the statistical analysis of 25-years of VEO data points, focusing on six key areas, clear trends emerge that can aid in forecasting the future VEO threat. Barring any significant and unexpected changes in societies, governments, and environments in the next 5 to 10 years, the statistical analysis shows that: 
- Afghanistan, Greece, India, Iraq, and Pakistan have a 75 to 100 percent chance of producing one or more VEOs;

- VEOs have a 77 percent chance of following either a nationalist or religious ideology;

- Armed assaults and bombings are the top two preferred attack methods; and,

- Private citizens and government entities are the top two preferred targets.

This study is broken down into two main sections. The first section looks at and engages in statistical analysis on: the number of VEOs for each of the four main ideology types (nationalist, religious, left-wing, and rightwing), identifying the countries of VEO emergence, determining which and how many attack methods each VEO uses, finding out what subpopulations VEOs are targeting, and how often VEOs are disbanding. In the second section, the results from the statistical analysis in the first section serve as the basis for a VEO forecast in these same six areas for the next 5 to 10 years. This second section also includes a discussion on what the results of the statistical analysis on the VEO threat may mean for the United States. Before delving into the first of the two sections, the remainder of this introduction provides background on how this study defines VEOs and its methodological approach.

\section{Defining Violent Extremism}

A commonly accepted definition for VEOs does not yet exist within the United States and the international community; however, one was necessary to help frame the study before research began. iv This study found four definitions describing "violent extremism." All say in some form or fashion that violence is used to obtain effects in one or more of the following domains: Political, religious, social, and economic. ${ }^{\mathrm{v}}$ Two additional definitions were found by breaking down the term into its parts with "violent" defined as "the use of physical force [by an individual or group]" and "extremism" as "beliefs or ideas [of an individual or a group] that are outside the scope of what the majority believes is reasonable."vi Taking into consideration the six definitions reviewed a VEO is a group of people that uses physical force to cause harm, damage, or kill/eradicate someone/something to achieve one or more political, religious, social, or economic objectives that the majority subjectively considers to be outside the scope of what is reasonable and acceptable behavior and norms.

This study also recognizes that state and non-state-sponsored entities are both capable of engaging in violent extremism activity; therefore, this study seeks to provide sub-definitions to delineate the difference between the two types of VEOs. The Political Terror Scale (PTS) project from the University of North Carolina defines state-sponsored VEOs as, "Agents of the state [that] perpetuate, sanction, or order the violation of basic human 
rights to the physical integrity of a person. Examples of an agent of the state are law enforcement, military, private military contractors, intelligence agents as well as executives and members of executive agencies and bureaucracies."vii Research also shows that state-sponsored VEOs are known to receive from one or more countries funding and a safe base of operations in which to conduct activities. In direct contrast, research shows that non-state sponsored VEOs are groups that at times use a decentralized leadership structure. They are known to have significant political influence but are not allied to states (that is, countries) and receive no state support.viii As demonstrated by these definitions, the primary difference between state-sponsored and non-state-sponsored VEOs is the presence or lack thereof of government support of their activities.

\section{Methodology}

The methodology employed for this study is a mixed-method approach that primarily focuses on the quantitative aspect in the form of statistical analysis, with qualitative data providing contextual support to the results. This study uses line graphs, column charts, maps, and tables to convey the results of the statistical analysis conducted in six areas: (1) years of VEO emergence; (2) ideology type; (3) location of VEO emergence; (4) attack types; (5) target types; and, (6) year of disbandment. The data for these six areas is derived solely from two entities that have a long-running history of tracking VEO information and activity: The University of Maryland Global Terrorism Database (UMD GTD) and the Terrorism Research and Analysis Consortium (TRAC).

The first order of business for this study was compiling a working dataset on which to base the statistical analysis from the 3,000-plus entities found within UMD GTD and TRAC from 1970 (est.) to 2017. There are five criteria used to compile a working dataset of 1,393 VEOs believed to be representative of the VEO threat. The first criterion focuses on determining the length of time necessary to identify apparent trends; this study assessed 25 years was enough time. The most recent and complete data from the UMD GTD and TRAC appears to be in the year 2016, so entities from these databases had to have an estimated emergence date starting sometime in 1992 up to and including 2016. ix The second criterion requires the entity to be easily identifiable as having one of the four main ideology types (nationalist, religious, left-wing, or right-wing) as other identified ideologies such as narcoterrorism and criminal are typically not under the domain of U.S. national security-focused organizations (that is, foreign intelligence and defense agencies). Based on available descriptions, subjective assessments were required to select a primary ideology type when more than one ideology type was present. The third criterion requires the entities to have emerged outside the United States; however, there are notations for foreign-based VEOs that were also emerging within 
the United States. ${ }^{\mathrm{x}}$ This exclusion is because this study's focus is on providing a forecast that possesses the potential to support U.S. national security decision-makers. A study focusing on U.S.-based VEOs in support of a different audience (that is, law enforcement) may be a possibility in the future. The fourth criterion excludes any entities that appear to be lone wolves or small cells of a larger entity; for example, the Bahrun Naim cell is a smaller cell within the Islamic State in Indonesia. Since group size is not available within UMD GTD and TRAC, this determination was also a subjective one. The fifth and final criterion requires data in the previous four areas to be available for consideration; automatic exclusions occurred for any entities in which the data was not available.

This study also elected to compile data points from the Failed State Index (FSI), as it is a reputable data source amongst U.S. national intelligence and defense agencies on assessing the stability of a country and ultimately the potential for VEO emergence. A less well-known source, the PTS, has the potential to serve the same purpose of ascertaining from where VEOs are likely to emerge. Compilation of this data also takes place to provide yet another point of reference. For those unfamiliar with the PTS methodology, it focuses on assessing the level of political terror within a given country by soliciting ratings from the U.S. State Department, Amnesty International, and the Human Rights Watch based on specific criteria. ${ }^{x i}$ As the PTS ratings come from three organizations, the numbers were averaged out to provide one data point instead of three for easy comparison against the single FSI rating given for each year. The compilation of the dataset occurs in a way that allows for easy comparison of the FSI and PTS ratings with each other and the associated number of emerging VEOs for a country in a given year. One important caveat: the dataset contains PTS data starting in 1992 up to and including 2016, while FSI data is only available starting in 2006 up to and including 2016.

Compilation of the data from each of these datasets occurred either within Microsoft Access or Excel to allow for more natural filtering of the information. For example, filtering the data for each of the years surveyed in the VEO dataset gave the results for the number of VEOs emerging in a given year. This study applies the same technique to each of the six areas, with a focus on separating the analysis by ideology type, and these results are then subject to statistical analysis. In the case of the FSI and PTS dataset, filtering occurred by rating, with data from 2006 to 2016 only, to see if each is providing lower or higher ratings for a country in a specific year, and then identify which ratings may be prone to VEO emergence. The statistical analysis in each of the six areas as well as the FSI and PTS resulted in clear trends that were then used to create a forecast of the VEO threat for the next 5 to 10 years.

This study presents the results and forecasts of the six different areas in the same numbered order in which they are listed earlier in this section. 
The idea is to start with a broad overview and then provide more specific results either by U.S. combatant command (CCMD) or ideology type. This study uses geographic U.S. CCMDs to discuss locations of VEO emergence as it allows for grouping of countries to identify broad trends common to a region. Differentiating, according to U.S. CCMD, also has the added benefit of highlighting specific trends to those U.S. military commanders responsible for managing or responding to a threat in a particular region. The segregation of results also occurs by each of the four common ideology types to show the preferred attack and target preferences, and disbandment frequency as specific nuances exist between the four types. Finally, the forecast section also includes a qualitative discussion that explores what the statistical analysis may mean for the United States concerning the VEO threat.

\section{Trend Analysis of Violent Extremist Organizations}

This section of the study focuses on conducting statistical analysis on the number of VEOs emerging each year (that is, timeline analysis); ideology types (nationalist, religious, left-wing, or right-wing); locations of VEO emergence; attack methods; targets; and disbandment. The focus is on identifying trends that evolved over 25 years in six areas to provide insight into the future VEO threat. In addition to the data derived from the VEO dataset, this section also incorporates the FSI and PTS datasets to see if these ratings, in isolation or combination, can provide indications of where VEOs are likely to emerge.

\section{Timeline and Ideology Analysis}

Based on the 1,393 VEOs included in the compiled dataset, the majority of the 25-year timespan indicates VEOs tend to emerge at a rate between 40 and 80 VEOs per year with an average of 56 VEOs per year (Figure 1). There were four years below this rate of emergence $(1993,2010,2015$, and 2016), which could be attributable to either an anomaly or the result of incomplete data for those specific years within the compiled dataset.xii Conversely, there was a slight increase above this rate of emergence in 1992 and 2014. The war in Bosnia and Herzegovina in 1992 as well as the Iraqi Civil War and the war in Donbas in 2014, may account for this slightly higher rate of emergence. ${ }^{\text {xii }}$ 
Figure 1. Timeline of Emerging VEOs over 25-Year Period

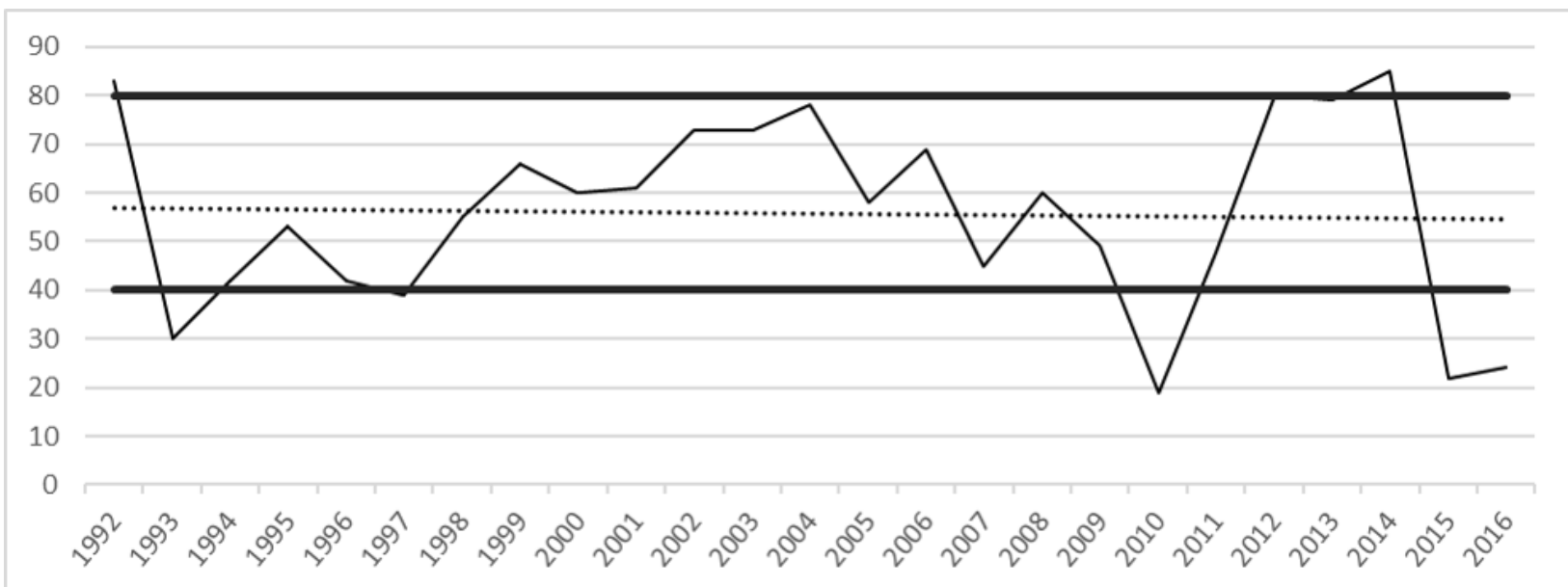

Notes. The researcher used the dataset created for this study to develop the data points for this chart. Source: Author.

Calculated percentages of the types of VEOs over the 25-year timespan reveal a higher probability exists of a VEO following a nationalist (41 percent) or religious (36 percent) ideology (Figure 2). This probability means a significant gap exists between nationalist and religious VEOs and those predominately associated with left-wing (18 percent) or right-wing (5 percent) ideologies. Although there are no calculations on the exact percentages, VEOs identified with multiple ideologies tended to be a combination of nationalist and religious ideologies instead of the other potential ideological combinations (that is, left-wing and religious, leftwing and nationalist, and so forth). The significant disparity within the VEO types is likely attributable to the perceived impact that the values of nationalist and religious ideologies have on the quality of life (both physical and spiritual) on participants. 
Figure 2. Ideological and Timeline Analysis Charts

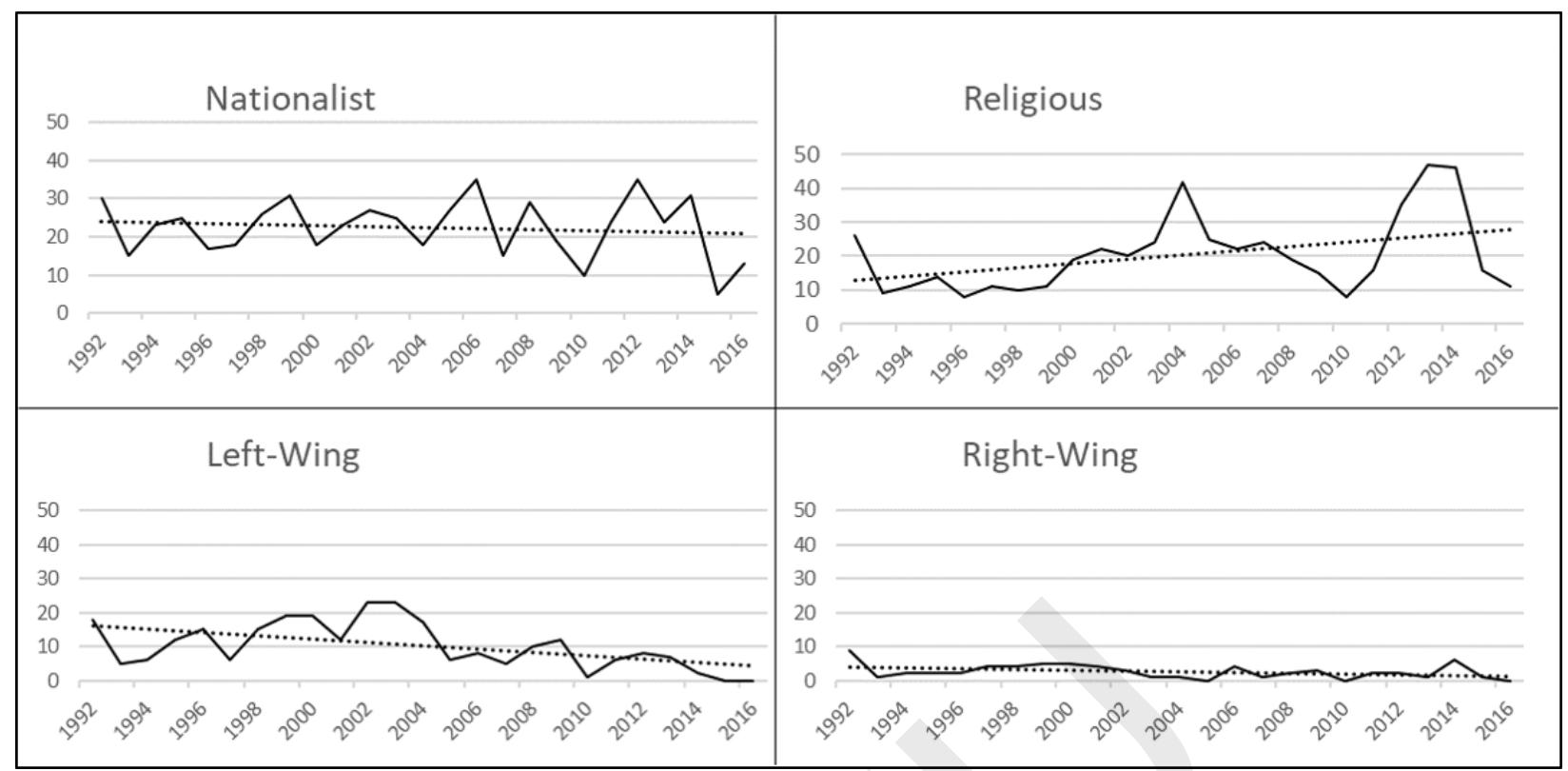

Notes. The researcher used the dataset created for this study to develop the data points for this graphic. Source: Author.

The calculations of percentages by year provide additional in-depth analysis of the type of emerging VEOs, revealing the following: Nationalist VEOs were dominant from 1992-1999, 2001-2003, 2005-2006, 20082011, and 2016; nationalist and religious VEOs were the same in 2012; and, religious VEOs were dominant in 2000, 2004, 2007, and 2013-2015 (Figure 2). However, it appears that the higher percentage of emerging religious VEOs in 2000 and 2007 is the direct result of a decrease in the number of nationalist VEOs as the number of emerging religious VEOs is consistent with previous years (that is, 1992-1999 and 2005-2006). In 2004 and 2012-2015, there was a significant rise in the number of emerging religious VEOs that reflects the higher percentages, which is likely attributable to the U.S. invasion of Iraq in 2003 as well as the subsequent partial withdrawal of U.S. troops from Iraq in 2011 and Afghanistan in 2014. ${ }^{\text {xiv }}$

\section{Location Analysis}

Trend analysis on the locations of VEOs does not provide a distinction between areas of VEO emergence and areas of operation, as this information was not consistently available within the UMD GTD and TRAC databases. Based on the VEOs surveyed in the working dataset, the majority of VEOs (75 percent) studied were operating in only one country, with the remaining 25 percent operating in two or more countries. Of those operating in two or more countries, religiously motivated VEOs 
accounted for half, followed by nationalist VEOs at 43 percent, left-wing at 4 percent, and right-wing at 3 percent. Additional insight is provided by engaging in analysis using the ratings provided by the FSI and PTS, by specific U.S. CCMD areas of responsibility (AOR), and countries of emergence/operation.

For a quick review, the FSI seeks to assess the stability of a country and ultimately the potential for its collapse using four bins that contain numerical ranges. The FSI scores reveal that in 8 percent of the instances reviewed, the country was considered sustainable (o-29), 20 percent stable (30-59), 51 percent warning (60-89), and 20 percent alerting (90-120) for the 10-year timespan reviewed. Of interest is that the analysis of FSI data shows that well over half of the countries in the warning and alerting categories are not seeing VEOs emerge (Table 1). The lack of VEOs emerging in these two categories was highly unexpected as it is a common belief among the U.S. national intelligence and defense agencies that higher levels of government instability portend higher levels of VEO emergence. However, despite these unexpected results, the countries in the warning and alerting categories still possess a higher likelihood of five or more VEOs emerging within the corresponding year than the stable and sustainable categories.

Table 1. Failed State Index Rating and Number of VEOs Emerging

\begin{tabular}{cccccccc}
\hline FSI & O VEOs & 1 VEO & 2 VEOs & 3 VEOs & 4 VEOs & 5 VEOs & $\begin{array}{c}>5 \\
\text { VEOs }\end{array}$ \\
\hline Sustainable & $86 \%$ & $11 \%$ & $3 \%$ & - & - & - & - \\
\hline Stable & $89 \%$ & $7 \%$ & $2 \%$ & $1 \%$ & $1 \%$ & - & - \\
\hline Warning & $85 \%$ & $8 \%$ & $2 \%$ & $2 \%$ & $1 \%$ & $1 \%$ & $1 \%$ \\
\hline Alerting & $65 \%$ & $18 \%$ & $8 \%$ & $2 \%$ & $2 \%$ & $1 \%$ & $4 \%$
\end{tabular}

Notes. The FSI uses four groups of indicators (cohesion, economic, political, and social) with the calculated results falling into one of four categories: Sustainable 0-29.9, Stable 3059.9, Warning 60-89.9, and Alerting 90-120.0. The basis for the percentages in this table is from the FSI scores and information on the emerging VEOs from the compiled dataset. Source: Author.

As stated previously, the PTS seeks to assess the level of terror perpetrated by a government against its population using a numbered rating system with a one indicating there is no political terror present and a five representing the highest level of political terror available. The PTS ratings reveal that in 26 percent of the instances, countries received a rating of one, 32 percent a rating of two, 26 percent a rating of three, 12 percent a rating of four, and 4 percent a rating of five for the 10-year timespan reviewed. The results show that in the higher two PTS rating categories, the percentages reflect a marked decrease from the lower three rating categories (Table 2). The results also show that countries receiving one of 
the higher two PTS ratings possessed a higher probability of two or more VEOs emerging than those receiving a rating of three and below (Table 2). Although unexpected, the results from the PTS ratings are reasonably accurate in identifying which countries possessed a higher probability of VEO emergence.

Table 2. PTS Rating and Number of VEOs Emerging

\begin{tabular}{cccccccc}
\hline PTS & O VEOS & $\mathbf{1}$ VEO & $\mathbf{2}$ VEOS & $\mathbf{3}$ VEOS & $\mathbf{4}$ VEOS & $\mathbf{5}$ & $\begin{array}{c}>\mathbf{5} \\
\text { VEOs }\end{array}$ \\
\hline $\mathbf{1}$ & $92 \%$ & $5 \%$ & $2 \%$ & $1 \%$ & - & - & - \\
\hline $\mathbf{2}$ & $90 \%$ & $9 \%$ & $1 \%$ & - & - & - & - \\
\hline $\mathbf{3}$ & $81 \%$ & $11 \%$ & $4 \%$ & $2 \%$ & $1 \%$ & $1 \%$ & - \\
\hline $\mathbf{4}$ & $55 \%$ & $20 \%$ & $10 \%$ & $7 \%$ & $2 \%$ & $1 \%$ & $5 \%$ \\
\hline $\mathbf{5}$ & $39 \%$ & $20 \%$ & $10 \%$ & $7 \%$ & $6 \%$ & $1 \%$ & $17 \%$
\end{tabular}

Notes. The PTS assesses the level of terror perpetrated by a government by five rating areas. One: Countries are under a secure rule of law, there is no imprisonment for people sharing their views, torture is rare or exceptional, and political murders are sporadic. Two: There is a limited amount of incarceration for nonviolent political activity; however, few persons are affected, torture and beatings are exceptional, and political murder is rare. Three: There is extensive political imprisonment (or a recent history of such imprisonment), execution or other political murders, the brutality may be standard, and unlimited detention (with or without a trial) for political views is accepted. Four: Civil and political rights violations have expanded to more significant numbers of the population; murders, disappearances, and torture are a standard part of life and affect those who interest themselves in politics or ideas. Five: Terror has expanded to the whole population, and the leaders of these societies place no limits on the means or thoroughness with which they pursue personal or ideological goals. The basis of the percentages in this table is from the PTS ratings and information on the emerging VEOs from the compiled dataset. Source: Author.

Of note, there was a marked difference in the predictive analysis between the FSI and PTS ratings. There seems to be no direct one-for-one correlation between an FSI rating of "sustainable" and a PTS rating of one. If this were the case, then the percentages would be more in-line with each other even though one has four rating categories and the other has five.

Although there were instances where the FSI and PTS ratings were both on the higher or lower end, it seems as though a good deal more were on the opposite ends of the spectrum. A prime example to demonstrate this discrepancy are the five countries that received FSI ratings in the "warning" and "alerting" categories as well as PTS ratings of either a one or a two to describe the same point in time. Grenada, Macedonia, the Maldives, Serbia, and Sierra Leone all were assessed to be unstable and yet rarely did a VEO emerge in any of these countries. The competing results between the FSI and PTS datasets may be surprising, but the statistical analysis does show that the PTS is a much more reliable indicator of VEO emergence. Nonetheless, the use of other data points in combination with the PTS ratings should increase the reliability and accuracy of any VEO forecast. 
This study also looks at VEO emergence by grouping countries by geographic U.S. CCMD to provide a broader examination of the previous analysis on FSI and PTS ratings based on locations of emergence. By grouping the VEO threat by U.S. CCMD, supportive qualitative information on ongoing regional issues (such as conflicts that are assessed to be a contributing factor to the emergence of VEOs) is easier to compile and incorporate for discussion. Each of the U.S. CCMD sections occurs in successive order from the highest to the lowest level of VEO emergence activity: Central Command (CENTCOM), European Command (EUCOM), Africa Command (AFRICOM), Indo-Pacific Command (INDOPACOM), Southern Command (SOUTHCOM), and Northern Command (NORTHCOM). Figure 3 serves as a visual representation of the number of emerging VEOs from 1992-2016 for each country, which can also serve as a reference point for the following U.S. CCMD discussion.

Figure 3. Locations of VEO Emergence from 1992-2016

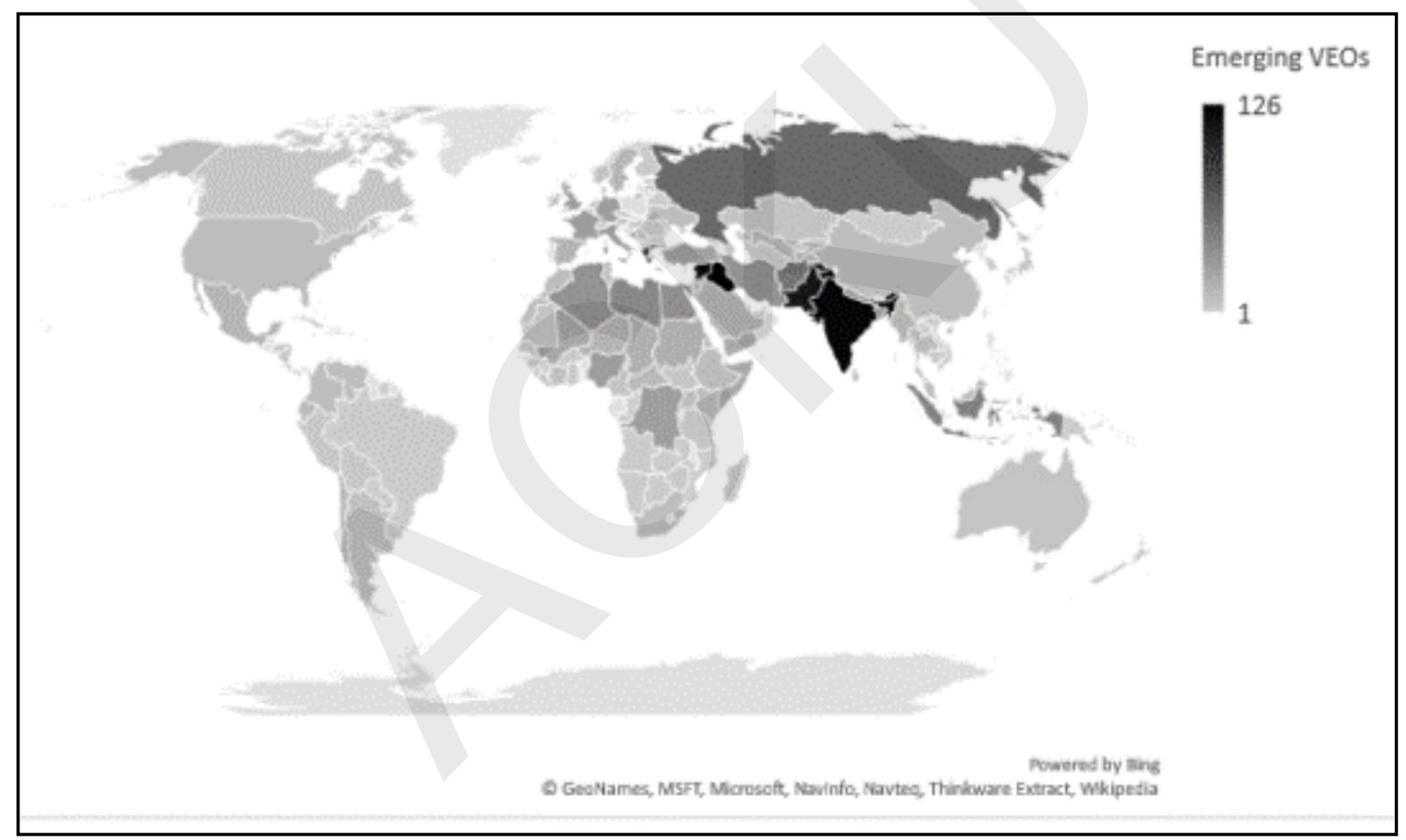

Notes. The researcher used the dataset created for this study to develop the data points for this graphic. This graphic only represents the number of emerging VEOs and not the total number of active VEOs. Source: Author.

Central Command: CENTCOM comprises the highest number of emerging VEOs in the last 25 years at 32 percent overall. Pakistan, Syria, and Iraq are home to 59 percent of the sub-total (over 100 VEOs each). Of the CENTCOM VEOs, 67 percent are religious-based, 32 percent nationalist, and the remaining 1 percent a combination of left-wing and right-wing groups. Iraq and Syria had the highest number of emerging VEOs in 2004 
and 2013 at 30 and 31 respectively, which is likely attributable to the U.S. invasion of Iraq in 2003, the subsequent partial U.S. withdrawal from Iraq in 2011, and the ramp-up to the start of the Iraqi Civil War in 2014. ${ }^{\mathrm{xv}}$ The relatively high number of emerging Pakistan VEOs is likely attributable to the ongoing conflict with India in the Kashmir region since 1947; however, Pakistan experienced a peak in 2007 of 13 emerging VEOs, but a specific contributing event is unknown.

European Command: EUCOM comprises the second-highest number of emerging VEOs at 25 percent overall. Germany, Turkey, France, Italy, Israel, Russia, and Greece are home to 78 percent of the sub-total (29-98 VEOs each). Of the EUCOM VEOs, 41 percent are nationalist based, 30 percent religious, 18 percent left-wing, and 11 percent right-wing. Greece has the highest overall number of emerging VEOs in the EUCOM AOR, the majority having left-wing ideologies (95 percent). Greece has a long, documented history of domestic political extremism, with periods of heightened violent outbursts by left-wing or right-wing VEOs. ${ }^{\text {xvi }}$ Russia also has a significant number of emerging VEOs over the 25-year timespan (62 VEOs), which is likely attributable to its two wars against Chechen separatists as well as the newly established presence of religious VEOs such as al-Qaeda and the Islamic State within the country..xvii

Africa Command: AFRICOM comprises the third-highest number of emerging VEOs at 20 percent overall. Somalia, Mali, the Democratic Republic of the Congo, Nigeria, Algeria, and Libya are home to 49 percent of the sub-total (23-42 VEOs each). Of the AFRICOM VEOs, 54 percent are nationalist-based, 41 percent religious, and the remaining 5 percent a mix of left-wing and right-wing groups. Libya peaked in 2014 at 10 emerging VEOs, with the remaining top countries contributing an average of 4 to 7 new VEOs each year. Libya has the highest number of emerging VEOs in the AFRICOM AOR, the majority of which emerged after the fall of Muammar Gadhafi in 2011. xvii Algeria is the second-highest source of emerging VEOs in the AFRICOM AOR. Emerging Algerian VEOs are primarily religious and have emerged consistently since the outbreak of the Algerian Civil War in 1992. ${ }^{\text {ix }}$

Indo-Pacific Command: INDOPACOM comprises the fourth-highest number of emerging VEOs at 18 percent overall. Nepal, Indonesia, and India are home to 58 percent of the sub-total. Of the INDOPACOM VEOs, 50 percent are nationalist-based, 35 percent religious, 14 percent leftwing, and 1 percent right-wing. India was the only country in the INDOPACOM region with over 100 new VEOs emerging in the 25-year timespan, which is likely attributable to border disputes with Pakistan over the Kashmir territory as well as domestic political extremism noted since gaining independence from British colonial rule in 1947.xx Nepal and Indonesia produced significantly fewer VEOs (less than 50 each). Although contributing information was not identifiable for Nepal-based 
VEOs, Indonesia's level of VEO emergence is likely attributable to the fall of the dictatorship of President Suharto in 1998.xxi

Southern Command: SOUTHCOM comprises the fifth-highest number of emerging VEOs, at 4 percent overall. Colombia, Ecuador, and Chile are home to 44 percent of the sub-total. Of the SOUTHCOM VEOs, 60 percent are left-wing based, 20 percent nationalist, 13 percent right-wing, and the remaining 7 percent religious. The top countries for the SOUTHCOM region produced 10-12 VEOs for the 25-year timespan. The numbers are relatively low, and there are no known studies which discuss the contributing factors for VEO emergence in these countries.

Northern Command: NORTHCOM comprises the lowest number of emerging VEOs, at 1 percent. Admittedly, this study did not include the number of domestic VEOs emerging within the United States, incorporation of which would likely still keep this AOR as the lowest emergence of VEOs. Taking into consideration the foreign-based VEOs that also emerged in the United States, 54 percent were left-wing based, 21 percent nationalist, and 25 percent religious. It is important to note here that VEOs do indeed emerge within the United States, and a relatively significant amount emerged within the 25-year timespan used for this study; for example, the right-wing VEOs such as the Alabama Free Militia and Imperial Clans of America.

\section{Attack Methods Analysis}

Research into the types of attacks executed by VEOs indicates they generally fall into one of seven categories: Armed assault, assassination, bombing/explosion, facility/infrastructure attack, hijacking, hostagetaking (kidnapping \& barricading), and unarmed assault. Of the 1,393 VEOs surveyed for this study, the preferred methods for all four ideology types are armed assault and bombing/explosion with a combined average of around 60 percent for each ideology type (Figure 4). VEOs rarely used assassination, hijacking, facility/infrastructure attacks, and unarmed assault within the 25-year timespan. Of the VEOs with known attack types, 54 percent used just one of the two preferred attack types; less than 1 percent (al-Shabaab, Communist Party of India-Maoist, People's Militia of the Donetsk People's Republic, Popular Resistance Committees, and the Taliban) used all seven attack types. Although VEOs can leverage a variety of attack types, the low cost and high impact of armed assaults and bombing/explosions are a winning combination for VEOs. 
Figure 4. Methods of Attack by VEO Type

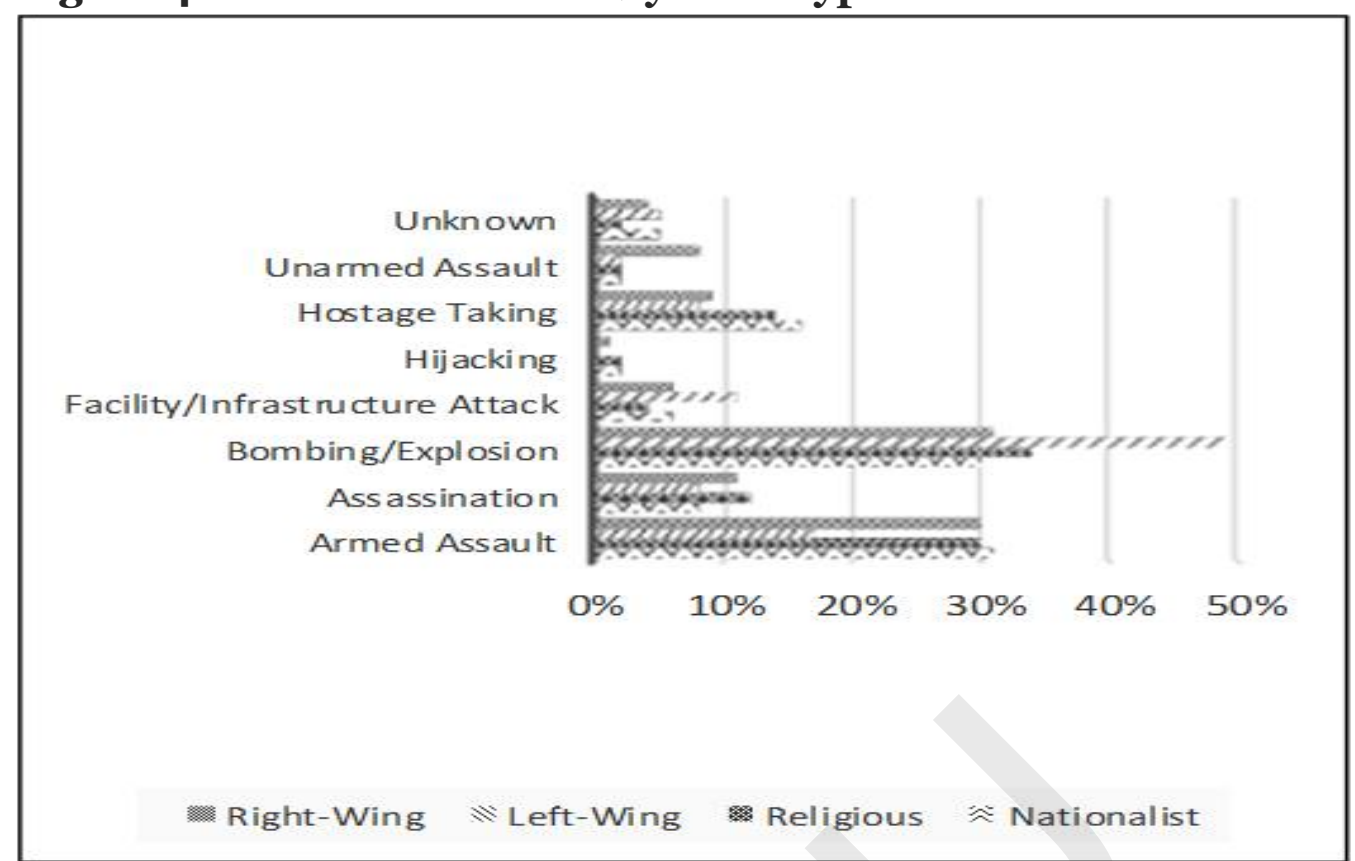

Notes. This bar chart represents the number of VEOs using a specific attack type within each of the VEO types (nationalist, religious, left-wing, and right-wing). Source: Author.

\section{Target Analysis}

Research into the types of VEO targets indicate they tend to fall into one of 20 categories: Airports and aircraft, businesses, educational institutes, food or water supply, government (diplomatic), government (general), journalists and media, maritime, military, non-governmental organizations (NGOs), police, private citizens/property, religious figures/institutions, telecommunications, terrorists/non-state militia, tourists, transportation, utilities, and violent political parties. Of the 1,393 VEOs surveyed for this study, the preferred targets for the four ideology types appear to be private citizens/property and government (general) (Figure 5). Religious and nationalist VEOs preferred to target the military in relatively high numbers. In addition, religious VEOs appear to have a higher probability of attacking other terrorists/non-state militias, which could be attributable to competition for scarce resources. Of the VEOs with known target types, 45 percent were attacking only one target type, with none using all 20 target types. However, 2 percent of the VEOs attacked 10-18 different target types, with al-Shabaab, Taliban, and Tehreek-eTaliban Islami Pakistan being on the higher end of that scale. 
Figure 5. Targets by VEO Type

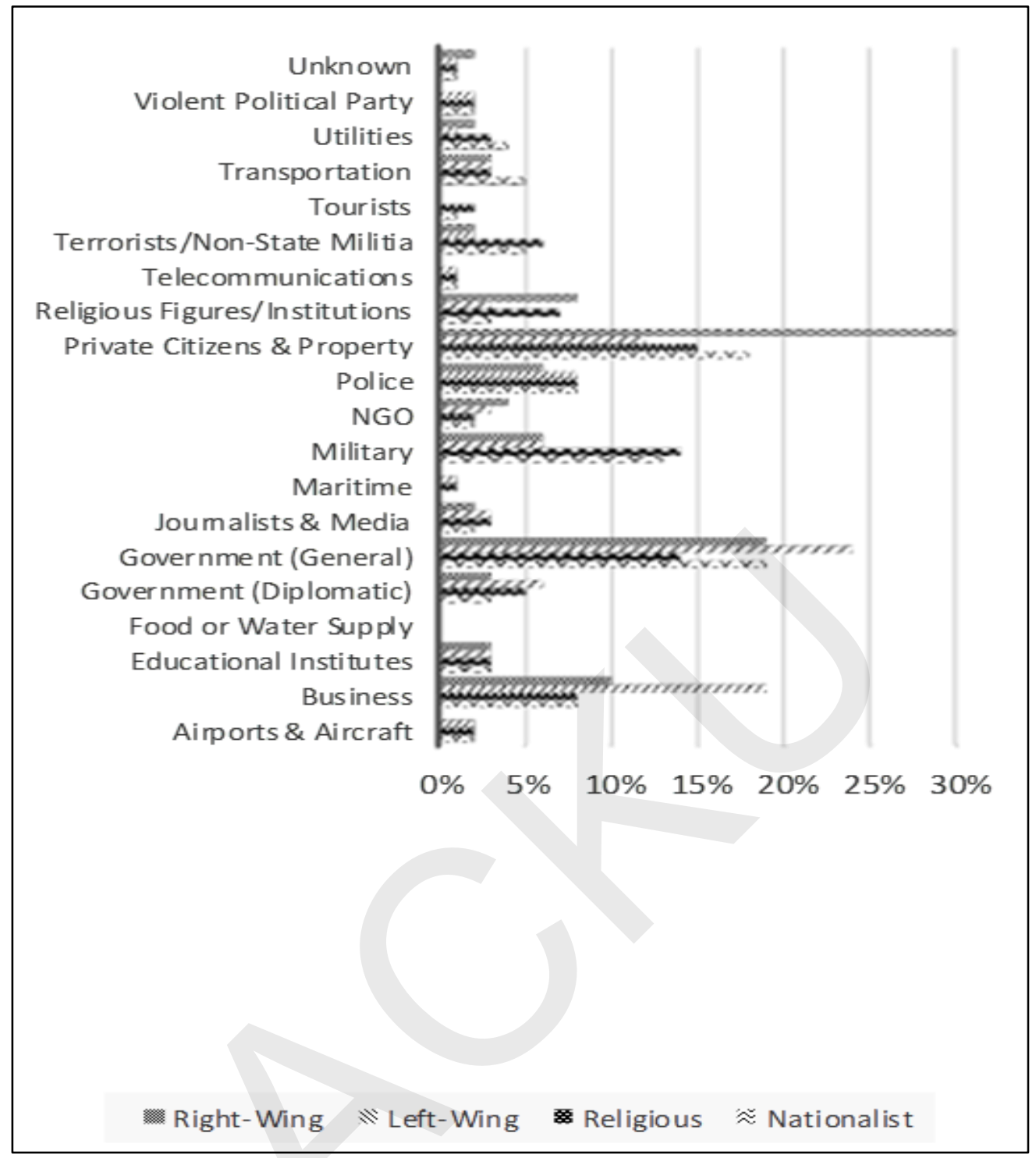

Notes. This bar chart represents the number of VEOs attacking a specific target type within each of the VEO types (nationalist, religious, left-wing, and right-wing). Source: Author.

Violent extremist organization targets may also be internal or external to their country or region of emergence/operation as evidenced by the attack against the U.S. World Trade Center and numerous attacks against U.S. entities within the CENTCOM AOR. Of the VEOs surveyed, the majority (75 percent) contained their attacks within one country with some attacking symbols of a foreign power within their area of interest. By their very definition, transnational VEOs are more likely than others to plan an attack against a target outside of their operating areas, but such attacks require significant planning and are quite costly. Although attacks such as 
the one occurring on 9/11 are possible, writ large VEOs focus on targets where they maintain a significant presence.

\section{Disbanding, Merging, and Reforming Trends}

For this iteration, this study does not conduct a comprehensive review to determine which of the 1,393 VEOs included in the working dataset may have disbanded, merged, or reformed since their initial emergence. That said, little to no information is readily available on disbandment dates and there is no ready broadly based information on VEOs merging or reemerging under new names. However, of the 1,393 VEOs in the dataset, 34 percent within the UMD GTD or TRAC appear to be inactive and given the classification of "disbanded." Left-wing VEOs had the highest rate of disbandment at 61 percent, followed by right-wing VEOs at 44 percent, nationalist at 31 percent, and religious at 24 percent (Figure 6). Of those, 73 percent of left-wing VEOs ended within the first year of formation, followed by religious at 70 percent, nationalist at 56 percent, and rightwing at 50 percent. The relatively high percentages of VEOs disbanding within the first year across all four ideology types is likely attributable to the lack of available resources to maintain and support the VEOs' objectives.

\section{Figure 6. Percentage of Duration of VEOs w/Estimated Disbandment Dates}

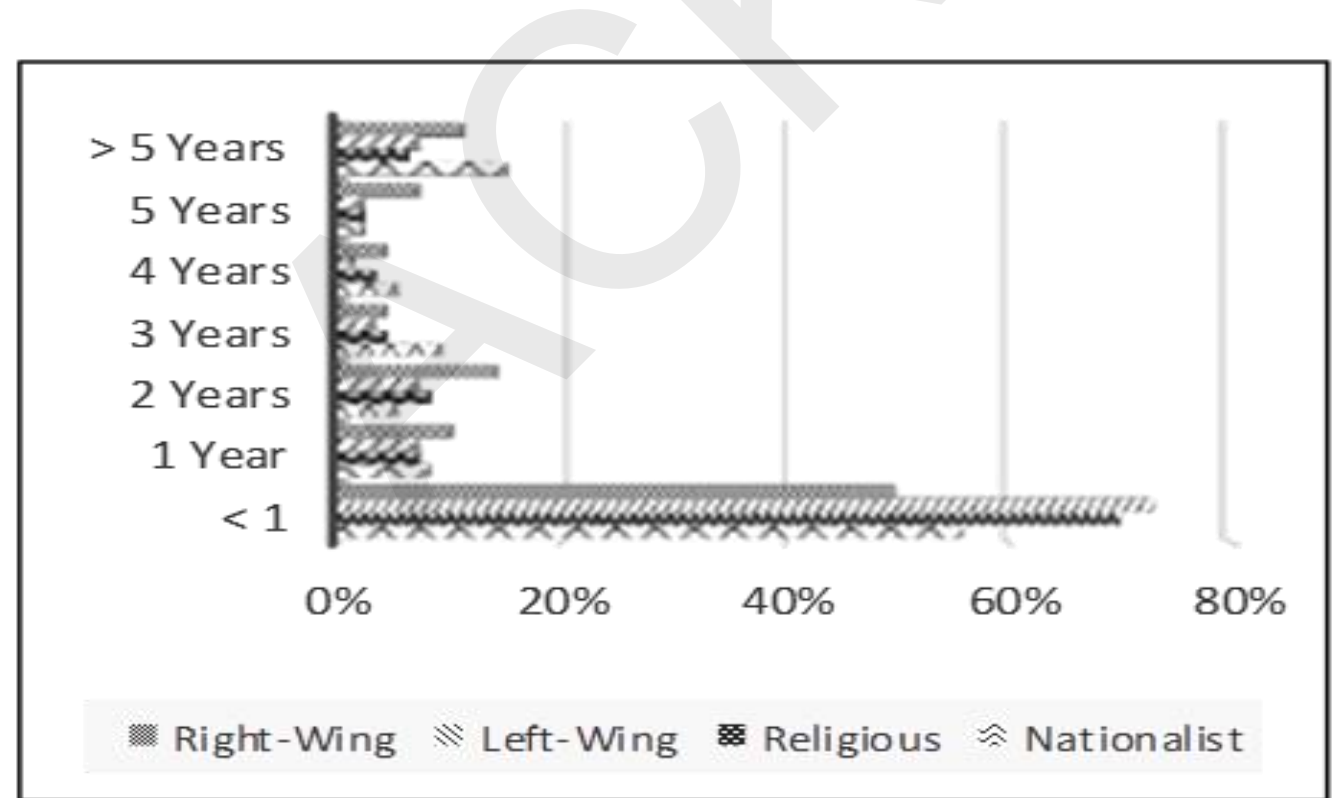

Notes. This bar chart represents the estimated duration of VEOs within the four different VEO types. The basis for the disbandment estimates is the lack of documented activity for the past 10 years in the UMD GTD or receiving the classification of inactive by the TRAC. The year of last known activity serves as the estimated disbandment date. Source: Author. 


\section{Future of Violent Extremist Organizations}

This section of the study focuses solely on providing an estimate on the number of VEOs that may emerge in the next 5 to 10 years, including how many are likely to be nationalist, religious, left-wing, or right-wing. This section also includes a forecast on the locations of emergence based on the PTS and U.S. CCMD AORs. The consistent trends in these areas for 25years, such as the predominance of religious VEOs emerging from the CENTCOM AOR using armed assault against private citizens/property, indicate they are likely to remain so for at least the next 5 to 10 years.

\section{Timeline and Ideology Forecast}

VEOs are likely going to continue emerging at a rate between 40 and 80 per year with an average rate of $56 \mathrm{VEOs} \mathrm{per} \mathrm{year.} \mathrm{This} \mathrm{rate} \mathrm{means} \mathrm{in} \mathrm{the}$ next 5 years, anywhere from 200-400 VEOs may emerge (280 represents the average rate), and in the next 10 years, anywhere from $400-800$ (560 represents the average rate). Significant events such as a conflict, war, or withdrawal of military troops are likely to result in deviations above the stated range in any given year. Deviations below the stated range are also likely should regions become more stable politically, socially, or economically. It is important to note that these ranges are not representative of the potential number of active VEOs, as just because they emerge does not mean they will remain active.

Delving into the four ideology types (nationalist, religious, left-wing, and right-wing), the majority of emerging VEOs are going to continue being either nationalist or religiously focused. An increasing number of VEOs are likely to combine nationalist and religious ideologies to appeal to a broader audience in the hopes of becoming more sustainable and prosperous in the near- and long-term. In comparison, left-wing and rightwing VEOs will either maintain their relatively low numbers or decline in the next 5 to 10 years even as they continue to emerge in higher numbers in certain countries such as Greece or the United Kingdom. These percentages (nationalist 40 percent, religious 36 percent, left-wing 18 percent, and right-wing 5 percent) are the basis for a calculated forecast for the number of VEOs emerging by ideology type in the next 5 to 10 years (Table 3). In the next 5 to 10 years, based on these percentages, there is also a possibility that more emerging VEOs will be focused on religious issues and at some point surpass nationalist VEOs as the dominant ideology type. 
Table 3. Forecast of Estimated Number of Emerging VEOs by Type

\begin{tabular}{lcc}
\hline & Over 5 Years & Over 10 Years \\
\hline Nationalist & $82-164$ (112 Average) & $164-328$ (224 Average) \\
\hline Religious & $72-144$ (100 Average) & $144-288$ (200 Average) \\
\hline Left-Wing & $36-72$ (50 Average) & $72-144$ (100 Average) \\
\hline Right-Wing & $10-20$ (14 Average) & 20-40 (28 Average)
\end{tabular}

Notes. The researcher used the estimated emerging rate of VEOs and the trend analysis percentages of nationalist, religious, left-wing, and right-wing VEOs to determine how many VEOs from each type may emerge in the next 5 to 10 years. Source: Author.

\section{Location Forecast}

The majority of VEOs are likely going to continue to be constrained to a single country, even though they might desire to conduct operations in two or more countries. Although some VEOs have "franchises," these franchises typically do not operate outside their local area of emergence (for example, ISIS-Philippines). VEOs primarily operating in just one country is likely going to remain a relatively steady trend for the next 5 to 10 years. However, the potential exists for VEOs to increasingly operate in two or more countries with the help of international platforms in the cyber domain (for example, Facebook, Twitter, and Telegram) to launch recruitment drives and establish cells within other locales. Finally, VEOs operating in two or more countries are going to continue to mostly follow either nationalist or religious ideologies due to the inherent desire to either protect and expand territorial boundaries or spread religious values.

Looking at the locations of VEO emergence by U.S. CCMD AOR, CENTCOM has historically produced the highest number of emerging VEOs. This trend is likely to continue in the next 5 to 10 years, followed by VEOs emerging in the EUCOM, AFRICOM, INDOPACOM, SOUTHCOM, and NORTHCOM AORs. The dominant types of VEOs for the CENTCOM (religious), EUCOM (nationalist), INDOPACOM (nationalist), SOUTHCOM (left-wing), and NORTHCOM (left-wing) AORs are likely to remain consistent in the next 5 to 10 years as well. In the AFRICOM AOR, however, the historical percentages indicate there is a possibility that religiously motivated VEOs may become the dominant ideology type in the next 5 to 10 years due to the importance of religious identification and perceived aggrieved status in the region.

Ascertaining the specific countries in each U.S. CCMD that are likely to produce one or more VEOs in the next 5 to 10 years is more challenging. As demonstrated in an earlier section, receiving a PTS rating of four or five does not necessarily guarantee a country is going to produce a VEO. Further illustrating the inability of the PTS to serve as a standalone forecasting tool is the example of Greece which had a high level of VEOs 
emerge from 2006-2016 with a PTS rating of either a three or below for those years. However, a PTS rating of four or five still possesses a somewhat higher reliability than the FSI in providing indications of which countries are going to see the emergence of three or more VEOs within a year.

Looking at the PTS ratings for each of the countries from 2006 to 2016, they appear to be relatively consistent and are likely to remain so barring any significant changes in societies, governments, and environments. ${ }^{x x i i}$ Based on the PTS ratings, 38 countries are likely to receive a PTS rating of either four or five in the next 5 to 10 years (Table 4). A rating of four or five means these countries are either expanding the violations of their civil and political rights within the targeted population or are exporting terror to its entire population, which likely makes them highly susceptible to VEO emergence.

Table 4. Forecasted Countries with PTS Rating of 4 or 5

\begin{tabular}{c|llll}
\hline $\begin{array}{c}\text { PTS } \\
\text { Rating }\end{array}$ & \multicolumn{3}{|c}{ Countries } \\
\hline $\mathbf{5}$ & Afghanistan & $\begin{array}{l}\text { Congo, Democratic } \\
\text { Republic }\end{array}$ & Iraq & Korea, Democratic \\
& Republic & & Sri Lanka \\
& Myanmar (Burma) & Somalia & South Sudan & \\
\hline $\mathbf{4}$ & Sudan & Syria & & Central African Republic \\
& Bangladesh & Brazil & Burundi & Eritrea \\
& China & Colombia & Egypt & Israel \\
& Ethiopia & India & Iran & Nepal \\
& Kenya & Mali & Mexico & Palestine \\
& Niger & Nigeria & Pakistan & Thailand \\
& Philippines & Russia & Saudi Arabia & Yemen \\
& Turkey & Uganda & Venezuela & \\
& Zimbabwe & & & Sem \\
\hline
\end{tabular}

Notes. The researcher used the dataset created for this study to calculate the PTS mode for each country to determine which countries received a PTS rating of four or five the most often from 2006 to 2016. Source: Author.

Because using the PTS cannot be done in isolation, this study also completes a year-by-year analysis of each country to determine how often VEOs are emerging. This year-by-year analysis looks at how many years from 2006-2016 a country experiences VEOs emerging in order to assess the probability of VEOs doing so in the future. This process identified 51 countries with a 25 to 91 percent chance for VEO emergence (Figure 7). In comparing these results with the forecasted PTS ratings, 18 countries have a higher probability of multiple VEOs emerging within a year:

Afghanistan, Bangladesh, the Central African Republic, the Democratic 
Republic of the Congo, Egypt, India, Iraq, Israel, Mali, Niger, Nigeria, Pakistan, Palestine, Philippines, Russia, Somalia, Sudan, and Syria.xxiii

\section{Figure 7. Countries Forecasted to Produce VEOs}

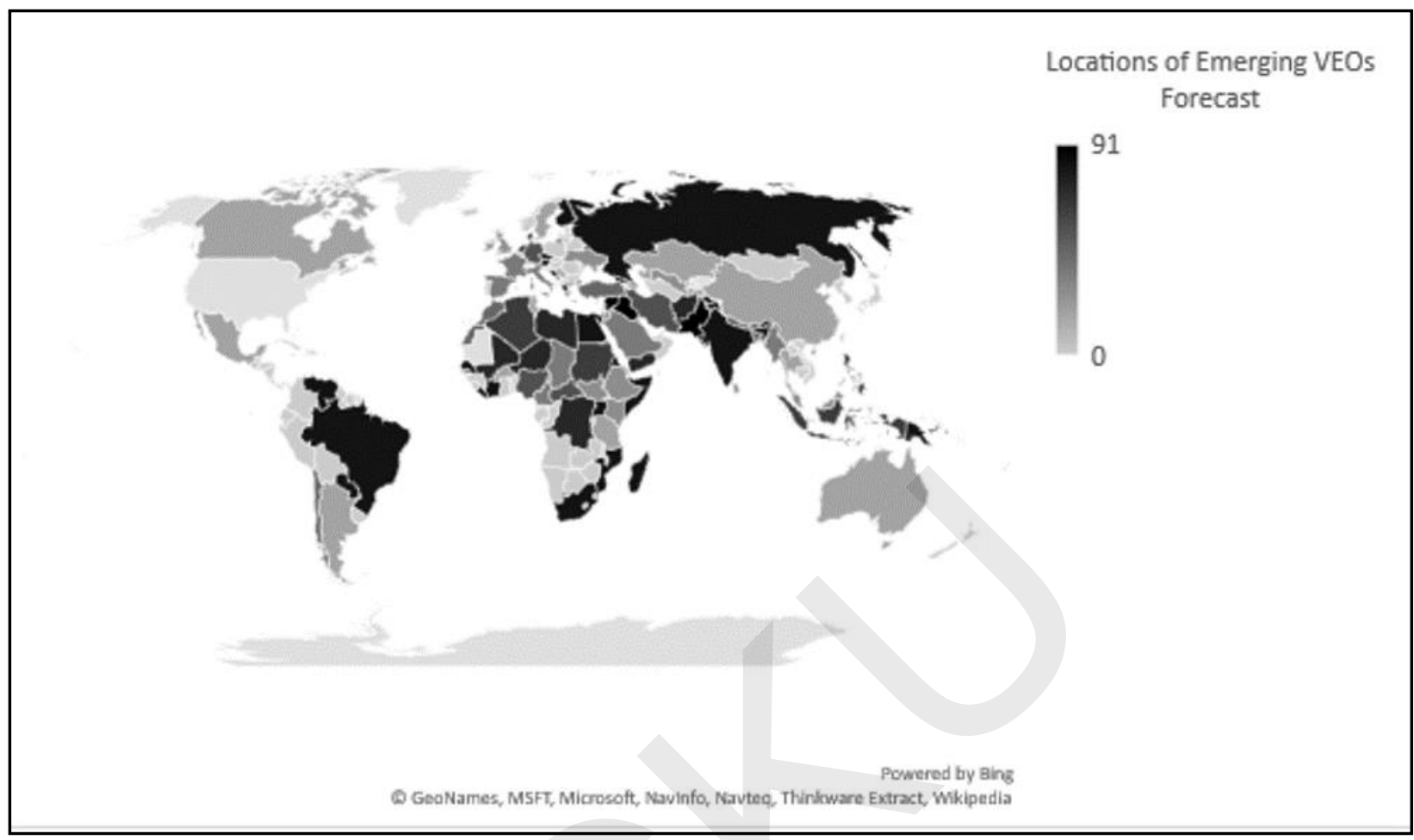

Notes. The researcher used the dataset created for this study to identify how often VEOs emerged in a specific country from 2006-2016. The percentages are the result of dividing the number of years a VEO emerged in a country by the number 11 (the number of years reviewed). For example, a country that has one or more VEOs emerging for 10 out of the 11 years would have a 91 percent chance of continuing this trend for the next 5 to 10 years. Source: Author.

\section{Attack Methods Forecast}

VEOs will likely continue to maintain focus on executing violent attacks that provide a medium to high level of collective psychological terror to assist them in achieving their stated objectives. Of crucial importance is the ability of VEOs to maximize available resources while still achieving the desired level of terror. VEOs are likely to prefer using armed assaults and bombing/explosion as their primary methods of conducting an attack. It is essential, however, to note that this does not exclude other attack methodologies (known and unknown) as VEOs can be creative in identifying new means of attack (for example, planes as bombs such as in the 9/11 attacks) and in capitalizing on presented opportunities. Although this study does not conduct extensive research in this area, there exists a possibility that the more attack types a VEO can leverage, the higher the 
likelihood that the size of the VEO is significant and likely to last more than a year.

\section{Target Forecast}

VEOs historically prefer targets they assess are going to provide the highest collective psychological impact and bring them closer to achieving their objectives. High levels of collective psychological terror are typically associated with attacks against private citizens/property as well as the government. As a result, these are likely going to continue to be the targets of choice for all four ideological types of VEOs. Additionally, military targets will likely be pursued more often by religious and nationalist VEOs, terrorists/non-state militias by religious VEOs, and businesses by left-wing VEOs. As previously discussed, VEOs may also focus on targets that are external to the country or region; however, VEOs desiring to attack foreign entities are likely to do so within a VEO's operating area (in other words foreign businesses, military bases, and government buildings located in that VEO's home of origin). Finally, those VEOs with a significant number of personnel at their disposal with placement and access in a variety of locations are more likely to be the ones engaging in attacks against more than one target type.

\section{Disbanding, Merging, and Reforming Forecast}

Despite the limited amount of information on VEOs disbanding, merging, and reforming within this study, left-wing and right-wing VEOs are more likely to disband in a shorter amount of time than nationalist and religious VEOs.xxiv Some of the emerging VEOs are likely to disband within the first year due to competition for scarce resources (in other words, people, money, and safehouses). However, calculating an exact percentage is difficult due to the limited amount of data in this area. Finally, it is difficult to forecast how many VEOs are going to disband, merge, or reform as many VEOs that have disbanded are now reemerging years later under the same name or adopting a new name while retaining their original objectives, targets, and methods of attack as before.

\section{What Does This Mean for the United States?}

This study provides what many may consider a relatively dire forecast, as it estimates that anywhere from 200-800 VEOs will emerge in the next 5 to 10 years. However, VEOs have consistently appeared on the international landscape at this rate, so the fact that they are likely to continue to do so should not be overly alarming. As history shows, not all VEOs that emerge pose a direct or indirect threat to the United States. For example, India has experienced a high number of VEOs (over 100) in the past 25 years, the majority of which are assessed to be associated with 
nationalist issues relating to its government or border disputes with Pakistan, but none of which have posed a significant threat to the United States to date. There must also be consideration given to the number of emerging VEOs that disband within the first year as a result of competition for scarce resources or due to successful local government and law enforcement intervention. Even though 200-800 VEOs may emerge, over the next 5 to 10 years, an equally high number is likely to disband, resulting in a relatively consistent number of active VEOs each year as opposed to active VEOs rising at a considerable rate.

In assessing the direct and indirect threat to the United States, there must be consideration given to the level of U.S. involvement (actual and perceived) in a foreign country's domestic, social and national security affairs. As demonstrated by the level of VEOs in India, the actual or perceived level of U.S. involvement is a critical factor in determining whether the U.S. is going to be a target. In the current environment, U.S. intelligence agencies assess VEOs with nationalist or religious ideologies pose the biggest threat to the United States as these ideologies have the highest probability of being more critical of perceived foreign intervention and influence. Research shows nationalist VEOs have historically targeted the United States because of military or diplomatic involvement in their country's affairs, whereas religious VEOs do so to stave off the potentially negative repercussions of external cultural norms. In either case, the perceived historical or current involvement of the United States in shaping the domestic affairs of a foreign country in ways the majority considers to be undesirable is likely to render it a target. For example, the United States' perceived involvement in shaping the Middle East in the areas of governmental and cultural norms has made it a target for most if not all VEOs emerging in the CENTCOM AOR.

In addition, the number of VEO operating locations is going to play an essential role in whether they pose a threat to the United States. The majority of VEOs are contained solely to one country and tend to direct activities at their respective governments. Unless those countries host U.S. military installations or commercial enterprises, the threat to the United States is likely minimal to non-existent. As a further limitation of their potential threat to the United States, the majority of these VEOs are unlikely to possess the resources to launch large-scale attacks outside their respective countries. However, transnational VEOs have a higher probability of possessing such resources and are therefore likely to pose anywhere from a minimal to a significant threat to the United States. In the next, 5 to 10 years, the most significant threat to the United States is likely going to appear from transnational VEOs operating in the CENTCOM, EUCOM, and AFRICOM regions due to the significant U.S. 
military presence and activities within them, and the negative perceptions some of these countries' populations may hold against the United States.

In expanding on the concept of transnational VEOs, research shows that VEOs falling under this descriptor are still primarily (but not exclusively) constrained to one country. These VEOs can portray the perception of operating in more than one country by becoming the franchisee of a VEO with a prominent brand name. This practice is evident with al-Qaida (for example, al-Qaida in the Arabian Peninsula, al-Qaida in Afghanistan, and al-Qaida in the Indian Subcontinent) as well as the Islamic State (for example, Islamic State in Algeria, Islamic State in Greater Sahara, and Islamic State in Libya). The franchisee VEO can capitalize on the brand name to help promote its group's objectives while still maintaining its independent leadership structure. In some cases, newly emerging and well-established VEOs are petitioning to use a particular VEO's brand name to capitalize on its popularity to acquire more resources within its operating location; for example, Salafist Group for Preaching and Fighting changed its name to al-Qaida in the Lands of the Islamic Maghreb. There are also numerous benefits for the VEO franchisor as the more VEO franchises it possesses, the more its notoriety and popularity rises as a result of a perceived expanded reach and attacks conducted under its brand name. Based on the compiled dataset for this study, $29 \mathrm{VEO}$ franchises were using the al-Qaida brand name and 24 VEO franchises were using the Islamic State brand name. However, it is currently unknown, without further in-depth research, how many were newly emerging VEOs versus how many were rebranding their organization. The al-Qaida and Islamic State franchisors are known to possess an inherent desire to target the United States, which means all the franchises will likely possess that same desire and are going to seek avenues to target the United States, limited only by the breadth and depth of their resources and skills.

Despite the existence of transnational VEOs and VEOs operating under a brand name, VEOs desiring and possessing the capability to target the United States are still relatively limited. The ability to attack the United States is dependent on available resources and where the VEOs can plan and execute attacks. As a result, VEOs rely heavily on the radicalization of "lone wolves" that possess the placement and access to execute attacks within the United States and countries that host U.S. interests (for example, military bases, large international corporations, a large population of American expatriates).

Research on VEOs indicate they tend to spend an inordinate amount of time on developing and distributing propaganda, in a multitude of 
languages, within highly popular social networking platforms such as Facebook, Twitter, and YouTube, to radicalize individuals in countries where they may not have a significant presence (for example, the United States). Although lone wolves do not possess the same level of resources as VEOs, they tend to have the advantage as most escape the notice of law enforcement agencies making it easier for them to acquire weapons and execute attacks. To increase the level of collective psychological terror as well as to inflate their placement and access globally, VEOs are known to claim responsibility for lone-wolf attacks, even though they are not directly involved in the planning and execution of the attacks.

\section{Conclusion}

This study presents a forecast that does not rely on overcomplicated methods while still providing slightly more detail than currently available forecasts. Researchers often overlook the most straightforward approach to assess a sophisticated threat, and yet as this study has proven, the most straightforward approach is still able to provide a great deal of insight. In comparing data that is objectively measurable; that is, (the number of VEOs emerging, ideology, locations of emergence and operation, attack methods, targets, and disbandment) and applying simple mathematical computations, trends and observations are easily identifiable. This study found that:

- Violent extremist organizations have a higher probability of emerging in countries whose governments are known to perpetrate terror against their populations (or subsets thereof), thereby creating a level of economic, political, and social instability.

- Violent extremist organizations have a higher probability of emerging in the CENTCOM region as most of the countries have experienced long-term instability. However, other countries such as India produce VEOs on the same level as Iraq, Pakistan, and Syria for the same reason.

- The ideology, attack methods, and targets of emerging VEOs appear to be relatively consistent within the same country or a U.S. CCMD's AOR over an extended period and are likely to remain so.

- Unless significant changes occur in the global environment, VEOs possess a higher probability of maintaining their current efforts and capabilities as well as scope, attack methods, and targets.

This study provides a solid and repeatable objective baseline forecast, and also opens up questions related to the PTS ratings and its potential correlation to how active or dangerous VEOs are in countries with a four 
or five rating. In essence, this study hopefully generated the interest of other researchers in the counterterrorism field to engage in research using simple, straightforward techniques in the development of forecasts that can be useful and impactful in generating plans to mitigate the threat in the near-term. Follow-on research to identify trends in a CCMD's AOR or a specific country would be useful. Additional efforts with a narrower focus would allow for increased scrutiny of the VEOs contained in the dataset as well as examine regional or country-specific factors that are contributing to VEO emergence.

\section{Endnotes}

i Edwin Bakker, "Forecasting Terrorism: The Need for a More Systematic Approach," Journal of Strategic Security 5, no. 4 (2012): 69-84, http://dx.doi.org/10.5038/19440472.5.4.5; Brynjar Lia, Globalisation and the Future of Terrorism: Patterns and Predictions (New York: Routledge, 2005); Gary Jackson, Predicting Malicious Behavior: Tools and Techniques for Ensuring Global Security (Indiana: John Wiley \& Sons, Inc 2012); Ibrahim Toure, "Real Time Big Data Analytics for Predicting Terrorist Incidents," PhD diss., (University of Maryland, 2016), http://dx.doi.org/10.1109/THS.2016.7568906.

ii Bakker, "Forecasting Terrorism: The Need for a More Systematic Approach"; Lia, "Globalisation and the Future of Terrorism: Patterns and Predictions"; Jackson, "Predicting Malicious Behavior: Tools and Techniques for Ensuring Global Security"; Toure, "Real Time Big Data Analytics for Predicting Terrorist Incidents".

iii Martin Gonzalez, "Terrorism," Office of the Director of National Intelligence, 2012, https://www.dni.gov/index.php/key-global-trends/terrorism.

iv Searches for VEO definitions only generated results for violent extremism definitions. Those identified were from the Federal Bureau of Investigation, as well as several others from a foreign government entity and independent research organizations. Other U.S. agencies such as the National Counterterrorism Center, Department of State, Department of Defense, or Department of Homeland Security did not have any definitions for VEOs. v "What is Violent Extremism," Federal Bureau of Investigation, Accessed July 2018, https://cve.fbi.gov/whatis/; "Evidence: Security of Women \& Security of States," May 2017, www.futureswithoutviolence.org; Andrew Glazzard and Martine Zeuthen, "Violent Extremism," GSDRC, February 2016, http://gsdrc.org/professional-dev/violentextremism/; "What is Violent Extremism?” Living Safe Together. Helping Communities Counter Violent Extremism, accessed July 2018, https://www.livingsafetogether.gov.au/aboutus/pages/what-is-violent-extremism.aspx. vi Merriam-Webster, s.v. "violent (n)," accessed July 2018, https://www.merriamwebster.com/dictionary/violent; Oxford Dictionary, violent (n)," accessed July 2018, https://en.oxforddictinaries.com/definition/violent.; Merriam-Webster, s.v. "extremism (n)," accessed July 2018, https://www.merriam-webster.com/dictionary/extremism.; Cambridge Dictionary, "extremism (n)," accessed July 2018, https://dictionary.cambridge.org/dictionary/english/extremism. This study focuses on 
identifying VEOs rather than individuals ("lone-wolves") who execute violent extremist acts based on ideologies similar to those of a VEO, without officially belonging to a VEOs. vii Peter Haschke, “The Political Terror Scale (PTS) Codebook" October 10, 2017, http://www.politicalterrorscale.org. viii "Non-State Actor," Oxford Dictionary, Accessed July 2018, https://en.oxforddictionaries.com/definition/non-state_actor/. ix This study does not differentiate from new or re-emerging VEOs. If an entity had a different name then it was treated as a new VEO as additional research did not occur outside of the UMD GTD and TRAC.

$x$ This study identifies VEOs with links to multiple countries. In these instances, the VEO is considered to have emerged in each country as circumstances allow them to operate within more than one country.

xi Haschke, "The Political Terror Scale (PTS) Codebook.

xii A portion of the VEOs identified in the UMD GTD and TRAC database did not have an estimated year of emergence, or one of the four primary ideologies could not be attributable to the VEO in question. The lack of this information may have contributed to the lower numbers for 1993, 2010, 2015, and 2016.

xiii John R Lampe, “Bosnian Conflict: European History [1992-1995]," Britannica Encyclopedia, Accessed August 30, 2018, https://www.britannica.com/event/bosnianconflict; "Iraqi Civil War - 2014," Global Security, Accessed August 28, 2018, www.globalsecurity.org/military/world/war/iraq-civil-war-2014.html; "The War in Donbass - 10 Facts," Borgen Project, Accessed August 28, 2018, https://borgenproject.org/the-war-in-donbass-10-facts/.

xiv Amy Belasco, The Cost of Iraq, Afghanistan, and Other Global War on Terror Operations Since 9/11, December 2014, Congressional Research Service, https://fas.org/sgp/crs/natsec/RL33110.pdf.

xv "Iraqi Civil War - 2014," Global Security; Belasco, The Cost of Iraq, Afghanistan, and Other Global War on Terror Operations Since 9/11.

xvi Othon Anastasakis, "The Far Right in Greece and the Theory of the Two Extremes," Open Democracy, May 2013, accessed September 2018,

https://www.opendemocracy.net/othon-anastasakis/far-right-in-greece-and-theory-oftwo-extremes-o; Theodora Ralli, "Violent Extremism in Greece," Master's Thesis (Masaryk University, 2014), https://globalcampus.eiuc.org/bitstream/handle/20.500.11825/447/ralli.pdf. xvii Press Release - September 2018, Press Release - August 2018, and Press Release July 2018, "Russia: Extremism \& Counter-Extremism," Counter Extremism Project, June 2018, https://www.counterextremism.com/countries/russia.

xviii “Libya, Extremism, \& the Consequences of Collapse," January 2016, http://soufangroup.com/wp-content/uploads/2016/o1/TSG_Libya-Extremism-and-theConsequences-of-Collapse-Jan2016.pdf.

xix Press Release - September 2018, Press Release - August 2018, and Press Release July 2018, "Algeria: Extremism \& Counter-Extremism," Counter Extremism Project, December 2017, https://www.counterextremism.com/countries/algeria.

xx Press Release - September 2018, Press Release - August 2018, and Press Release July 2018, "India: Extremism \& Counter-Extremism," Counter Extremism Project, June 2018, https://www.counterextremism.com/countries/india. 
xxi Press Release - September 2018, Press Release - August 2018, and Press Release July 3018, "Indonesia: Extremism \& Counter-Extremism," Counter Extremism Project, August 2018, https://www.counterextremism.com/countries/indonesia.

xxii Other significant changes that would impact this forecast include, but are not limited to: Economic conditions, ecological disaster, social/religious upheaval, the overthrow of government leaders, institution of a new government type, invasion and/or occupation by a hostile power, major sustained depression with significant unemployment, drought/flooding/earthquake which causes major casualties and strains government's ability to provide basic services, or fundamentalist religious revolution.

xxii These countries have a higher probability of receiving a PTS rating of four or five as well as those countries noted with VEOs emerging more frequently. The countries identified in both lists likely have the highest probability of VEOs emerging. xxiv Seth Jones and Martin Libicki, "How Terrorist Groups End: Lessons for Countering al Qa'ida," 2008, RAND Corporation. The information identified in this study provides confirmation of the conclusions drawn in the study conducted by Jones and Libicki regarding the probability of nationalist and religious VEOs having a longer duration than left-wing and right-wing VEOs. 
Row 1: Average PTS Ratings

Appendix A

Row 2: FSI Ratings

Row 3: Number of Emerging VEOs

\begin{tabular}{|c|c|c|c|c|c|c|c|c|c|c|c|c|c|c|c|c|c|c|c|c|c|c|c|c|c|}
\hline & 1992 & 1993 & 1994 & 1995 & 1996 & 1997 & 1998 & 1999 & $\begin{array}{c}200 \\
\mathbf{0}\end{array}$ & $\begin{array}{c}200 \\
1\end{array}$ & $\begin{array}{c}200 \\
2\end{array}$ & $\begin{array}{c}200 \\
3\end{array}$ & $\begin{array}{c}200 \\
4\end{array}$ & $\begin{array}{c}200 \\
5\end{array}$ & $\begin{array}{c}200 \\
6\end{array}$ & $\begin{array}{c}200 \\
7\end{array}$ & $\begin{array}{c}200 \\
8\end{array}$ & $\begin{array}{c}200 \\
9\end{array}$ & $\begin{array}{c}201 \\
\mathbf{0}\end{array}$ & 2011 & 2012 & 2013 & 2014 & 2015 & 2016 \\
\hline \multirow[t]{3}{*}{ Afghanistan } & 4.5 & 4.5 & 5 & 5 & 4.5 & 5 & 5 & 5 & 5 & 5 & 4.5 & 4 & 4.5 & 5 & 5 & 5 & 5 & 5 & 4.5 & 5 & 4 & 5 & 4.5 & 4.5 & 4.5 \\
\hline & - & - & - & - & - & - & - & - & - & - & - & - & - & - & 99.8 & 102.3 & 105.4 & 108.2 & 109.3 & 107.5 & 106 & 106.7 & 106.5 & 107.9 & 107.9 \\
\hline & 6 & o & 1 & 1 & 2 & 1 & 2 & 4 & 3 & 4 & 1 & 3 & 2 & o & o & 6 & 4 & 3 & 1 & 2 & 2 & 3 & 3 & o & o \\
\hline \multirow[t]{3}{*}{ Albania } & 2 & 2 & 2.5 & 2.5 & 2.5 & 4 & 2.5 & 3 & 2.5 & 2.5 & 3 & 2 & 2.5 & 2.5 & 2.5 & 2.5 & 2 & 2 & 2 & 2 & 2 & 2 & 2 & 2.5 & 2 \\
\hline & - & - & - & - & - & - & - & - & - & - & - & - & - & - & 68.6 & 70.5 & 69.7 & 70 & 67.1 & 66.1 & 66.1 & 65.2 & 63.6 & 61.9 & 61.2 \\
\hline & o & o & o & o & o & o & o & 1 & o & o & o & o & 0 & o & o & o & o & o & o & o & o & o & o & o & o \\
\hline \multirow[t]{3}{*}{ Algeria } & 4 & 4.5 & 5 & 5 & 5 & 5 & 5 & 5 & 4.5 & 4.5 & 4 & 4.5 & 4 & 4 & 4 & 3.5 & 3 & 2.5 & 3 & 3 & 2 & 2.5 & 2.5 & 2.5 & 2.5 \\
\hline & - & - & - & - & - & - & - & - & - & - & - & - & - & - & 77.8 & 75.9 & 77.8 & 80.6 & 81.3 & 78 & 78.1 & 78.7 & 78.8 & 79.6 & 78.3 \\
\hline & 6 & 4 & 1 & 1 & o & 3 & 1 & 1 & 2 & o & 1 & 1 & 1 & o & 3 & 2 & 1 & o & o & 2 & 1 & 1 & 4 & o & o \\
\hline \multirow[t]{3}{*}{ Angola } & 4.5 & 5 & 5 & 4.5 & 4.5 & 4 & 5 & 5 & 4.5 & 4.5 & 4.5 & 4 & 4 & 3.5 & 3 & 3.5 & 3 & 3.5 & 3 & 3 & 3 & 3.5 & 3.5 & 3 & 2.5 \\
\hline & - & - & - & - & - & - & - & - & - & - & - & - & - & - & 88.3 & 84.9 & 83.8 & 85 & 83.7 & 84.6 & 85.1 & 87.1 & 87.4 & 87.9 & 90.5 \\
\hline & 0 & 0 & 0 & 0 & o & 0 & 0 & 0 & 0 & 0 & 0 & 1 & 0 & o & 0 & 0 & 0 & 0 & 0 & 0 & 0 & o & 0 & o & o \\
\hline \multirow[t]{3}{*}{ Argentina } & 2 & 2 & 2 & 2 & 2.5 & 2 & 2.5 & 2.5 & 2 & 3 & 2.5 & 2 & 2.5 & 2 & 2 & 2 & 2 & 2.5 & 2 & 2.5 & 1.5 & 2 & 2.5 & 2 & 2 \\
\hline & - & - & - & - & - & - & - & - & - & - & - & - & - & - & 40.8 & 41.4 & 41.4 & 44.7 & 45.8 & 46.8 & 46.5 & 46.1 & $47 \cdot 3$ & 47.6 & 48.4 \\
\hline & 1 & 1 & o & o & o & o & o & o & o & o & o & o & 1 & 2 & o & o & o & o & o & 1 & 1 & o & o & o & o \\
\hline \multirow[t]{3}{*}{ Armenia } & 2 & 2 & 2 & 2 & 2 & 2.5 & 2.5 & 2.5 & 2 & 2 & 3 & 2 & 2.5 & 2 & 2 & 2.5 & 3 & 2.5 & 2.5 & 3 & 2.5 & 2.5 & 3 & 2.5 & 3 \\
\hline & - & - & - & - & - & - & - & - & - & - & - & - & - & - & 71.5 & 70.3 & 70.7 & $74 \cdot 3$ & 74.1 & 72.3 & 72.2 & 71.3 & 71.3 & 69.7 & 69.6 \\
\hline & 2 & 1 & o & 1 & o & o & o & o & o & o & o & o & o & o & o & o & o & o & o & o & o & o & o & o & o \\
\hline \multirow[t]{3}{*}{ Australia } & 1 & 1 & 1 & 1 & 1 & 1 & 1 & 1 & 1 & 1 & 1.5 & 2 & 1.5 & 1.5 & 1 & 1 & 1 & 1 & 1.5 & 1 & 1 & 1 & 1.5 & 1.5 & 1.5 \\
\hline & - & - & - & - & - & - & - & - & - & - & - & - & - & - & 22 & 23.2 & 24.6 & $25 \cdot 9$ & 27.3 & 28.1 & 29.2 & 25.4 & 26.3 & $24 \cdot 3$ & 22.5 \\
\hline & 1 & o & o & 1 & o & o & o & o & o & o & o & o & 1 & o & 1 & o & o & 1 & o & o & o & o & o & o & o \\
\hline \multirow[t]{3}{*}{ Austria } & 1 & 1 & 1 & 1 & 1 & 1 & 1 & 1 & 1 & 1 & 2 & 2 & 2 & 1.5 & 2 & 2 & 1.5 & 2 & 1.5 & 1 & 1 & 1 & 1 & 1.5 & 1 \\
\hline & - & - & - & - & - & - & - & - & - & - & - & - & - & - & 26.1 & 26 & 25.9 & 27.6 & 27.2 & 27.3 & 27.5 & 26.9 & 28.5 & 25.8 & 27.5 \\
\hline & o & o & o & 3 & o & 1 & 1 & o & o & o & o & o & o & o & o & o & o & 1 & o & o & o & o & o & o & o \\
\hline \multirow[t]{3}{*}{ Azerbaijan } & 4 & 4 & 3.5 & 3 & 2.5 & 2.5 & 2 & 2 & 2.5 & 2 & 2.5 & 2.5 & 2.5 & 3 & 2 & 3 & 2.5 & 2.5 & 3 & 3 & 3 & 3 & 3 & 3 & 3 \\
\hline & - & - & - & - & - & - & - & - & - & - & - & - & - & - & 81.9 & 81.2 & 81 & 84.6 & 84.4 & 81.9 & 79.8 & 78.2 & 77.8 & $77 \cdot 3$ & 76.3 \\
\hline & 1 & 1 & 1 & 0 & o & 0 & 1 & 1 & o & 0 & 1 & o & o & o & o & 1 & 1 & 1 & o & o & o & o & o & o & 0 \\
\hline
\end{tabular}




\begin{tabular}{|c|c|c|c|c|c|c|c|c|c|c|c|c|c|c|c|c|c|c|c|c|c|c|c|c|c|}
\hline & 1992 & 1993 & 1994 & 1995 & 1996 & 1997 & 1998 & 1999 & $\begin{array}{c}200 \\
\mathbf{0}\end{array}$ & $\begin{array}{c}200 \\
1\end{array}$ & $\begin{array}{c}200 \\
2\end{array}$ & $\begin{array}{c}\mathbf{2 0 0} \\
\mathbf{3}\end{array}$ & $\begin{array}{c}200 \\
4\end{array}$ & $\begin{array}{c}200 \\
5\end{array}$ & $\begin{array}{c}200 \\
6\end{array}$ & $\begin{array}{c}200 \\
7\end{array}$ & $\begin{array}{c}200 \\
8\end{array}$ & $\begin{array}{c}200 \\
9\end{array}$ & $\begin{array}{c}\mathbf{2 0 1} \\
\mathbf{0}\end{array}$ & 2011 & 2012 & 2013 & 2014 & 2015 & 2016 \\
\hline \multirow[t]{3}{*}{ Bahamas } & 1 & 1 & 1 & 1 & 1 & 1 & $\overline{1}$ & 1 & 1 & 1 & 2 & 2 & 1.5 & 1.5 & 1.5 & 2.5 & 2 & 2 & 1.5 & 2 & 2 & 2 & 2 & 2.5 & 2 \\
\hline & - & - & - & - & - & - & - & - & - & - & - & - & - & - & - & 60.1 & 60.5 & 60.9 & 58.9 & 56.5 & 55.1 & $54 \cdot 7$ & 54.1 & 51.5 & 51.6 \\
\hline & o & o & o & o & o & o & o & o & o & o & o & o & o & o & o & o & o & o & o & o & o & o & o & o & o \\
\hline \multirow[t]{3}{*}{ Bahrain } & 2 & 2 & 2.5 & 3.5 & 3 & 2.5 & 2.5 & 2.5 & 2.5 & 1 & 2 & 1 & 1 & 1.5 & 1.5 & 2 & 2 & 1.5 & 2.5 & 3 & 3 & 3 & 3 & 3 & 3 \\
\hline & - & - & - & - & - & - & - & - & - & - & - & - & - & - & - & 57 & 56.8 & 59 & 58.8 & 59 & 62.2 & 62.9 & 64.7 & 64.4 & 63.4 \\
\hline & o & o & o & o & o & o & o & o & o & o & o & o & o & o & o & o & o & o & o & 1 & 1 & 3 & 3 & o & o \\
\hline \multirow[t]{3}{*}{ Bangladesh } & 4 & 3 & 3 & 3 & 3.5 & 3.5 & 3 & 3 & 3 & 3.5 & 3.5 & 3.5 & 4 & 4 & 4 & 4 & 4 & 4 & 4 & 3.5 & 3.5 & 4 & 4 & 4 & 4 \\
\hline & - & - & - & - & - & - & - & - & - & - & - & - & - & - & 96.3 & $95 \cdot 9$ & 100. & 98.1 & 96.1 & 94.4 & 92.2 & 92.5 & 92.8 & 91.8 & 90.7 \\
\hline & 5 & o & 3 & 5 & 3 & o & 3 & o & 1 & 2 & 3 & o & 2 & o & 1 & 3 & & 1 & o & o & 2 & o & 1 & o & o \\
\hline \multirow{3}{*}{ Barbados } & 1 & 1 & 1 & 1 & 1 & 1 & 1 & 1 & 1 & 1 & 1 & 1 & 2 & 2 & 2 & 2 & 2 & 2 & 2 & 2 & 1 & 1 & 1 & 2 & 2 \\
\hline & - & - & - & - & - & - & - & - & - & - & - & - & - & - & - & 59.9 & 58.5 & 57.2 & $55 \cdot 4$ & 52.8 & 52 & 50.8 & 51.4 & 49.3 & 49 \\
\hline & o & o & o & o & o & o & o & o & o & o & o & o & o & o & o & o & o & o & o & o & o & o & o & o & o \\
\hline \multirow{3}{*}{ Belarus } & 2 & 2 & 1.5 & 2 & 2 & 3 & 2.5 & 2.5 & 3 & 2.5 & 2.5 & 2.5 & 3 & 2.5 & 3 & 2.5 & 3 & 2.5 & 2.5 & 2.5 & 2.5 & 3 & 2.5 & 2.5 & 2.5 \\
\hline & - & - & - & - & - & - & - & - & - & . & - & - & - & - & 84.5 & 85.2 & 84.4 & 82.3 & 78.7 & 77.6 & 76.6 & 76.7 & 75 & 75.6 & 73.9 \\
\hline & o & o & o & o & o & 1 & o & o & o & o & o & o & o & o & o & o & o & o & o & o & o & o & o & o & o \\
\hline \multirow{3}{*}{ Belgium } & 1 & 1 & 1 & 1 & 1 & 1 & 1 & 1 & 1 & 1 & 1.5 & 1.5 & 1.5 & 1.5 & 1.5 & 1.5 & 1.5 & 1.5 & 1.5 & 1 & 1 & 1 & 1 & 1 & 1 \\
\hline & - & - & - & - & - & - & - & - & - & - & - & - & - & - & 24 & 25.5 & 29 & 33.5 & 32 & 34.1 & 33.5 & 30.9 & 32 & 30.5 & 29 \\
\hline & o & o & o & 1 & o & o & 3 & 0 & o & o & 0 & o & o & 2 & 1 & o & o & o & o & 1 & o & o & o & o & o \\
\hline \multirow[t]{3}{*}{ Belize } & 1 & 1 & 1 & 1 & 1 & 1 & 1 & 1 & 1 & 1 & 2.5 & 2 & 2 & 2.5 & 2 & 2 & 2 & 3 & 2 & 2 & 2 & 2 & 2 & 2 & 2 \\
\hline & - & - & - & - & - & - & - & - & - & - & - & - & - & - & - & 69.8 & 70.2 & 69.5 & 68.7 & 67.7 & 67.2 & 67.2 & 67 & $65 \cdot 3$ & 66 \\
\hline & o & o & o & o & o & o & o & o & o & o & o & o & o & o & o & o & o & o & o & o & o & o & o & o & o \\
\hline \multirow[t]{3}{*}{ Benin } & 1.5 & 1.5 & 1.5 & 2 & 1 & 1 & 2 & 2 & 2 & 2 & 2 & 2 & 2 & 3 & 3 & 2 & 2.5 & 2 & 2 & 2 & 1.5 & 2 & 2 & 2 & 2 \\
\hline & - & - & - & - & - & - & - & - & - & - & - & - & - & - & 70.9 & 72 & 72.8 & 75.5 & 76.8 & 80 & 78.6 & 77.9 & 78.2 & 78.8 & 78.9 \\
\hline & o & o & o & o & o & o & o & o & o & o & o & o & o & o & o & o & o & o & o & o & o & o & o & o & o \\
\hline \multirow[t]{3}{*}{ Bhutan } & 3.5 & 3.5 & 3.5 & 2 & 1.5 & 2.5 & 1.5 & 2 & 1.5 & 1.5 & 1.5 & 1 & 2 & 1 & 1 & 1 & 2 & 3 & 2 & 2 & 2 & 2 & 1 & 2 & 1 \\
\hline & - & - & - & - & - & - & - & - & - & - & - & - & - & - & 87.9 & 86.4 & 85.4 & 87.3 & 87.3 & 85 & 82.4 & 81.8 & 80.9 & 78.7 & 77.6 \\
\hline & o & o & o & 1 & 1 & o & o & o & o & o & o & 1 & o & o & o & o & 1 & o & o & o & o & o & 0 & o & o \\
\hline
\end{tabular}




\begin{tabular}{|c|c|c|c|c|c|c|c|c|c|c|c|c|c|c|c|c|c|c|c|c|c|c|c|c|c|}
\hline & 1992 & 1993 & 1994 & 1995 & 1996 & 1997 & 1998 & 1999 & $\begin{array}{c}200 \\
\mathbf{0}\end{array}$ & $\begin{array}{c}200 \\
1\end{array}$ & $\begin{array}{c}200 \\
2\end{array}$ & $\begin{array}{c}200 \\
3\end{array}$ & $\begin{array}{c}200 \\
4\end{array}$ & $\begin{array}{c}200 \\
5\end{array}$ & $\underset{6}{200}$ & $\begin{array}{c}200 \\
7\end{array}$ & $\begin{array}{c}\mathbf{2 0 0} \\
8\end{array}$ & $\begin{array}{c}200 \\
9\end{array}$ & $\begin{array}{c}201 \\
\mathbf{0}\end{array}$ & 2011 & 2012 & 2013 & 2014 & 2015 & 2016 \\
\hline \multirow[t]{3}{*}{ Bolivia } & 2.5 & 2.5 & 2 & 2 & 2 & 2 & 2 & 2.5 & 2.5 & 2 & 2.5 & 3 & 2.5 & 2.5 & 2.5 & 2.5 & 2.5 & 1.5 & 2 & 2 & 2 & 2 & 2 & 1.5 & 2 \\
\hline & - & - & - & - & - & - & - & - & - & - & - & - & - & - & 82.9 & 82 & 84.2 & 86.3 & 84.9 & 82.9 & 82.1 & 80.8 & 78.9 & 78.1 & 78.5 \\
\hline & 1 & o & o & o & o & o & o & o & o & o & o & 1 & o & 1 & o & o & o & o & o & o & o & o & o & o & o \\
\hline \multirow{3}{*}{$\begin{array}{l}\text { Bosnia and } \\
\text { Herzegovina }\end{array}$} & 5 & 5 & 5 & 5 & 3 & 3.5 & 3 & 3 & 3 & 2.5 & 2 & 2 & 2 & 2 & 2 & 2 & 2 & 2 & 2 & 1.5 & 1.5 & 2 & 2 & 1.5 & 2 \\
\hline & - & - & - & - & - & - & - & - & - & - & - & - & - & - & 88.5 & 84.5 & 84.3 & 83.3 & 83.5 & 80.9 & 77.9 & 76.5 & 75.9 & 77.4 & 74.6 \\
\hline & 6 & 2 & o & o & 1 & o & 1 & o & o & o & o & o & o & o & o & o & o & o & 2 & o & o & o & o & o & o \\
\hline \multirow[t]{3}{*}{ Botswana } & 1.5 & 1 & 1 & 1 & 1 & 1 & 1 & 1 & 1 & 1 & 2 & 2 & 2 & 2 & 2 & 2 & 2 & 2 & 1 & 1 & 2 & 1 & 1 & 1 & 2 \\
\hline & - & - & - & - & - & - & - & - & - & - & - & - & - & - & 66.9 & 66.4 & 65.9 & 68.8 & 68.6 & 67.9 & 66.5 & 64 & 64.5 & 62.8 & 63.5 \\
\hline & o & o & o & o & o & o & 1 & o & o & o & o & o & o & o & o & o & o & o & o & o & o & o & o & o & o \\
\hline \multirow[t]{3}{*}{ Brazil } & 4.5 & 4 & 4 & 4 & 4 & 3.5 & 3 & 4 & 3.5 & 4 & 4 & 4 & 4 & 4 & 4 & 4 & 4 & 4 & 4 & 3.5 & 4 & 4 & 4 & 4 & 3.5 \\
\hline & - & - & - & - & - & - & - & - & - & - & - & - & - & - & 63.1 & 66.9 & 67.6 & 69.1 & 67.4 & 65.1 & 64.1 & 62.1 & 61.4 & 62.7 & 65.3 \\
\hline & o & o & o & o & o & o & o & o & o & o & o & o & o & o & o & o & o & 1 & o & o & o & o & o & o & o \\
\hline \multirow[t]{3}{*}{ Brunei } & 1 & 1 & 2 & 2 & 1 & 1 & 1 & 2 & 1 & 1 & 2 & 2 & 1.5 & 1 & 1 & 1 & 1 & 1 & 1 & 1 & 1 & 1 & 1 & 1 & 1.5 \\
\hline & - & - & - & - & - & - & - & - & - & - & - & - & - & - & - & 71.2 & 68.5 & 68.1 & 67.6 & 65.8 & 64.1 & 63.2 & 63.6 & 63 & 62 \\
\hline & o & o & o & o & o & o & o & o & o & o & o & o & o & o & o & o & o & o & o & o & o & o & o & o & o \\
\hline \multirow[t]{3}{*}{ Bulgaria } & 2 & 2 & 2.5 & 2.5 & 2.5 & 2.5 & 2 & 3 & 2.5 & 2.5 & 2 & 2 & 2 & 2.5 & 2.5 & 2.5 & 2 & 2 & 2 & 2 & 1 & 2 & 2 & 2 & 2 \\
\hline & - & - & - & - & - & - & - & - & - & - & - & - & - & - & 62.1 & 60.3 & 58.5 & 61.5 & 61.2 & 59 & 56.3 & 55 & 54.4 & 55.4 & 53.7 \\
\hline & o & o & o & o & o & o & o & 1 & o & 1 & o & 0 & o & o & o & o & o & o & o & 0 & o & o & o & o & 0 \\
\hline \multirow[t]{3}{*}{ Burkina Faso } & 1.5 & 2 & 3 & 3 & 2 & 2 & 2 & 2.5 & 2 & 2 & 3 & 2 & 1.5 & 2 & 3 & 3 & 3 & 2 & 2 & 3 & 1.5 & 3 & 3 & 3 & 2.5 \\
\hline & - & - & - & - & - & - & - & - & - & - & - & - & - & - & 89.7 & 89.7 & 89.9 & 91.3 & 90.7 & 88.6 & 87.4 & 90.2 & 89 & 89.2 & 89.4 \\
\hline & o & o & o & o & o & o & 1 & o & o & o & o & o & o & o & o & o & o & o & o & o & o & o & o & 1 & 1 \\
\hline \multirow[t]{3}{*}{ Burundi } & 4 & 5 & 5 & 5 & 5 & 5 & 5 & 5 & 4.5 & 5 & 5 & 4 & 4.5 & 4.5 & 4 & 4 & 4 & 3 & 3.5 & 3.5 & 3.5 & 4 & 3 & 4 & 4 \\
\hline & - & - & - & - & - & - & - & - & - & - & - & - & - & - & 96.7 & 95.2 & 94.1 & 95.7 & 96.7 & 98.6 & 97.5 & 97.6 & 97.1 & 98 & 100.7 \\
\hline & 1 & 1 & o & 2 & o & o & o & o & o & o & o & o & o & o & o & o & 1 & o & 1 & o & o & o & o & o & o \\
\hline \multirow[t]{3}{*}{ Cambodia } & 3 & 4 & 4 & 3.5 & 3.5 & 3.5 & 3.5 & 3 & 2.5 & 2.5 & 3 & 3 & 3 & 3 & 3 & 3.5 & 3 & 3 & 3 & 3 & 3 & 3.5 & 3 & 2.5 & 2.5 \\
\hline & - & - & - & - & - & - & - & - & - & - & - & - & - & - & 85 & 85.7 & 85.8 & 87.3 & 88.7 & 88.5 & 88.7 & 88 & 88.5 & 87.9 & 87.4 \\
\hline & o & o & o & o & o & 1 & 1 & o & o & o & o & o & o & o & o & o & o & o & o & o & o & o & o & o & o \\
\hline
\end{tabular}




\begin{tabular}{|c|c|c|c|c|c|c|c|c|c|c|c|c|c|c|c|c|c|c|c|c|c|c|c|c|c|}
\hline & 1992 & 1993 & 1994 & 1995 & 1996 & 1997 & 1998 & 1999 & $\begin{array}{c}200 \\
0\end{array}$ & $\begin{array}{c}200 \\
1\end{array}$ & $\begin{array}{c}200 \\
2\end{array}$ & $\begin{array}{c}200 \\
3\end{array}$ & $\begin{array}{c}200 \\
4\end{array}$ & $\begin{array}{c}200 \\
5\end{array}$ & $\begin{array}{c}200 \\
6\end{array}$ & $\begin{array}{c}200 \\
7\end{array}$ & $\begin{array}{c}200 \\
8\end{array}$ & $\begin{array}{c}200 \\
9\end{array}$ & $\begin{array}{c}201 \\
\mathbf{0}\end{array}$ & 2011 & 2012 & 2013 & 2014 & 2015 & 2016 \\
\hline \multirow[t]{3}{*}{ Cameroon } & 3.5 & 3 & 4 & 3 & 3 & 3 & 3.5 & 4 & 4 & 3 & 3 & 4 & 3.5 & 3.5 & 3.5 & 3 & 4 & 2.5 & 2.5 & 2.5 & 2.5 & 3 & 3.5 & 3 & $3 \cdot 5$ \\
\hline & - & - & - & - & - & - & - & - & - & - & - & - & - & - & 88.4 & 89.4 & 91.2 & $95 \cdot 3$ & 95.4 & 94.6 & 93.1 & 93.5 & 93.1 & 94.3 & 97.8 \\
\hline & o & o & o & o & o & o & o & o & o & o & o & o & o & o & o & o & o & 1 & o & o & 3 & o & 1 & o & 2 \\
\hline \multirow[t]{3}{*}{ Canada } & 1 & 1 & 1 & 1 & 1 & 1 & 1 & 1 & 1 & 1 & 1.5 & 1 & 1 & 1 & 1 & 1.5 & 1.5 & 1 & 1 & 1 & 1 & 1 & 1 & 1 & 1 \\
\hline & - & - & - & - & - & - & - & - & - & - & - & - & - & - & 23.1 & 25.1 & 26.3 & 27.7 & 27.9 & 27.7 & 26.8 & 26 & 27.4 & 25.6 & 23.8 \\
\hline & 1 & o & o & o & o & o & o & o & 1 & o & o & o & o & o & o & o & o & 1 & o & o & 1 & o & o & o & o \\
\hline \multirow{3}{*}{$\begin{array}{l}\text { Cape } \\
\text { Verde/Cabo } \\
\text { Verde }\end{array}$} & 1 & 1 & 1 & 1 & 1 & 1 & 1 & 1 & 1 & 1 & 2 & 1 & 1 & 2 & 1 & 2 & 2 & 2 & 2 & 2 & 1 & 2 & - & 1 & 1 \\
\hline & - & - & - & - & - & - & - & - & - & - & - & - & - & - & - & 81.1 & 80.7 & 78.5 & 77.2 & 75.8 & 74.7 & 73.7 & 74.1 & 73.5 & 71.5 \\
\hline & o & o & o & o & o & o & o & o & o & o & o & o & o & o & o & o & o & o & o & o & o & o & o & o & o \\
\hline \multirow{3}{*}{$\begin{array}{l}\text { Central African } \\
\text { Republic }\end{array}$} & 2.5 & 2 & 2 & 2 & 2 & 3 & 2 & 3 & 2 & 4 & 3 & 4 & 3 & 3.5 & 4.5 & 4.5 & 4 & 4 & 4.5 & 4 & 4 & 5 & 5 & 4 & 3.5 \\
\hline & - & - & - & - & - & - & - & - & - & - & - & - & - & - & 97.5 & 101.0 & 103.7 & 105.4 & 106.4 & 105 & 103.8 & $105 \cdot 3$ & 110.6 & 111.9 & 112.1 \\
\hline & o & o & o & o & o & o & o & o & o & 1 & o & 2 & o & o & 1 & 1 & 2 & 2 & o & o & o & 2 & 1 & o & 0 \\
\hline \multirow[t]{3}{*}{ Chad } & 4.5 & 4.5 & 4 & 4 & 3 & 4 & 4 & 4 & 3.5 & 4 & 3 & 3.5 & 3.5 & 3 & 4 & 4 & 5 & 4.5 & 4 & 3.5 & 3 & 3 & 3 & 3 & 3.5 \\
\hline & - & - & - & - & - & - & - & - & - & - & - & - & - & - & 105.9 & 108 & 110.9 & 112.2 & 113.3 & 110.3 & 107.6 & 109 & 108.7 & 108. & 110.1 \\
\hline & 1 & o & 1 & o & o & o & 2 & o & o & 1 & o & 2 & o & o & 2 & o & 4 & o & o & o & 1 & o & o & & 1 \\
\hline \multirow[t]{3}{*}{ Chile } & 3 & 2.5 & 2 & 2 & 2 & 2 & 2 & 2 & 2 & 2 & 2 & 2 & 1 & 1 & 1.5 & 1.5 & 2 & 2 & 1.5 & 2 & 2 & 2 & 2 & 1.5 & 2 \\
\hline & - & - & - & - & - & - & - & - & - & - & - & - & - & - & 32 & 33.8 & 36.3 & 37.5 & 38 & 40.7 & 43.5 & 42.3 & 42 & 41.5 & 41.9 \\
\hline & 3 & o & o & o & o & o & 1 & 0 & o & o & 1 & 1 & o & o & 2 & o & 1 & 1 & o & o & o & 1 & o & o & 1 \\
\hline \multirow[t]{3}{*}{ China } & 3.5 & 3 & 3.5 & 3 & 3.5 & 4 & 4.5 & 4 & 4.5 & 4 & 4 & 4 & 4 & 4 & 4 & 3.5 & 4 & 4 & 4 & 4 & 4 & 4 & 4 & 4 & 4 \\
\hline & - & - & - & - & - & - & - & - & - & - & - & - & - & - & 82.5 & 81.2 & 80.3 & 84.6 & 83 & 80.1 & 78.3 & 80.9 & 79 & 76.5 & 74.9 \\
\hline & o & o & o & 1 & 2 & 2 & 1 & o & 1 & 1 & o & o & o & o & o & o & 1 & o & o & o & 1 & o & o & o & o \\
\hline \multirow[t]{3}{*}{ Colombia } & 4.5 & 5 & 5 & 4.5 & 5 & 5 & 5 & 5 & 5 & 5 & 5 & 5 & 5 & 4.5 & 4.5 & 4 & 4.5 & 4.5 & 4.5 & 4 & 3.5 & 4 & 3.5 & 3.5 & 3.5 \\
\hline & - & - & - & - & - & - & - & - & - & - & - & - & - & - & 91.8 & 89.7 & 89 & 89.2 & 88.2 & 87 & 84.4 & 83.8 & 83.1 & 82.5 & 80.2 \\
\hline & 1 & 1 & 1 & 2 & o & 1 & 1 & 1 & 0 & 1 & o & o & 1 & o & o & o & o & o & o & o & o & o & o & o & o \\
\hline \multirow[t]{3}{*}{ Comoros } & 2.5 & 2 & 2 & 2 & 1 & 2.5 & 1 & 3 & 2 & 2 & 1 & 2 & 1 & 1 & 1 & 3 & 3 & 1 & 1 & 2 & 2 & 1 & 1 & 1 & 1 \\
\hline & - & - & - & - & - & - & - & - & - & - & - & - & - & - & - & 77.8 & 79.6 & 86.3 & 85.1 & 83.8 & 83 & 84 & 85.1 & 83.3 & 83.8 \\
\hline & o & o & 0 & o & o & o & o & o & o & o & o & o & o & o & o & o & o & o & o & o & o & o & o & o & o \\
\hline
\end{tabular}




\begin{tabular}{|c|c|c|c|c|c|c|c|c|c|c|c|c|c|c|c|c|c|c|c|c|c|c|c|c|c|}
\hline & 1992 & 1993 & 1994 & 1995 & 1996 & 1997 & 1998 & 1999 & $\begin{array}{l}200 \\
\text { o }\end{array}$ & $\begin{array}{c}200 \\
1\end{array}$ & $\begin{array}{c}200 \\
2\end{array}$ & $\begin{array}{c}200 \\
3\end{array}$ & $\begin{array}{c}200 \\
4\end{array}$ & $\begin{array}{c}200 \\
5\end{array}$ & $\begin{array}{c}200 \\
6\end{array}$ & $\begin{array}{c}200 \\
7\end{array}$ & $\begin{array}{c}200 \\
8\end{array}$ & $\begin{array}{c}200 \\
9\end{array}$ & $\begin{array}{c}201 \\
\mathbf{0}\end{array}$ & 2011 & 2012 & 2013 & 2014 & 2015 & 2016 \\
\hline \multirow[t]{3}{*}{ Congo, Republic } & 2 & 3.5 & 4 & 3 & 3 & 5 & 4.5 & 5 & 4 & 3 & 4 & 4 & 2.5 & 2.5 & 2.5 & 2.5 & 3 & 2.5 & 2 & 2 & 2.5 & 3 & $3 \cdot 5$ & 4 & 4 \\
\hline & - & - & - & - & - & - & - & - & - & - & - & - & - & - & - & 93 & 93.4 & 93.1 & 92.5 & 91.4 & 90.1 & 90 & 89.6 & 90.8 & 92.2 \\
\hline & o & o & 1 & o & o & o & o & o & o & o & o & o & o & o & o & o & o & o & o & o & o & o & o & o & o \\
\hline \multirow{3}{*}{$\begin{array}{l}\text { Congo, the } \\
\text { Democratic } \\
\text { Republic of the }\end{array}$} & 4 & 5 & 4.5 & 4 & 4.5 & 5 & 5 & 5 & 5 & 4.5 & 5 & 5 & 5 & 4.5 & 4.5 & 5 & 5 & 5 & 5 & 4.5 & 5 & 5 & 4 & 4.5 & 4.5 \\
\hline & - & - & - & - & - & - & - & - & - & - & - & - & - & - & 110.1 & 105.5 & 106.7 & 108.7 & 109.9 & 108.2 & 111.2 & 111.9 & 110.2 & 109.7 & 110 \\
\hline & o & 3 & o & 2 & 1 & 1 & 4 & o & o & 2 & 1 & 2 & o & 1 & 2 & 2 & 1 & o & o & 1 & 2 & o & 1 & 1 & 1 \\
\hline \multirow[t]{3}{*}{ Costa Rica } & 1.5 & 1 & 1 & 1.5 & 1.5 & 1 & 1 & 1 & 1 & 1.5 & 1 & 1 & 4 & 1 & 1 & 1 & 2 & 2 & 1 & 2 & 2 & 2 & 2 & 1 & 2 \\
\hline & - & - & - & - & - & - & - & - & - & - & - & - & - & - & 49.6 & 50.5 & 50.9 & 52.5 & 52 & 50.6 & 49.7 & 48.7 & 48.5 & 46.8 & 45.1 \\
\hline & o & o & o & o & 1 & o & o & o & o & o & o & o & o & o & o & o & o & o & o & o & o & o & o & o & o \\
\hline \multirow{3}{*}{$\begin{array}{l}\text { Cote d'Ivoire } \\
\text { (Ivory Coast) }\end{array}$} & 3 & 2 & 2.5 & 2.5 & 3 & 2.5 & 2.5 & 2.5 & 3.5 & 2.5 & 4 & 4 & 4.5 & 4 & 4 & 4 & 3.5 & 3.5 & 3.5 & 4.5 & 4 & 3 & 3 & 3 & 3 \\
\hline & - & - & - & - & - & - & - & - & - & - & - & - & - & - & 109.2 & 107.3 & 104.6 & 102.5 & 101.2 & 102.8 & 103.6 & 103.5 & 101.7 & 100.1 & 97.9 \\
\hline & 1 & 1 & o & o & o & o & o & o & o & o & 3 & o & o & o & 2 & o & o & o & o & o & o & o & o & o & o \\
\hline \multirow[t]{3}{*}{ Croatia } & 4.5 & 4 & 3.5 & 5 & 3 & 3 & 3 & 3 & 1 & 2 & 2 & 2 & 2 & 2 & 2 & 1 & 1 & 1 & 1 & 1 & 1 & 1 & 1 & 1 & 1 \\
\hline & - & - & - & - & - & - & - & - & - & - & - & - & - & - & 61.9 & 60.5 & 59.4 & 60.1 & 59 & $57 \cdot 3$ & 56.3 & 54.1 & 52.9 & 51 & 52.4 \\
\hline & o & o & o & o & o & o & 1 & o & o & 1 & o & o & o & o & o & o & o & o & o & o & o & o & o & o & o \\
\hline \multirow[t]{3}{*}{ Cuba } & 3 & 3 & 3 & 3.5 & 3 & 3 & 3 & 3 & 3 & 3 & 2.5 & 2.5 & 2.5 & 2.5 & 3 & 3 & 3 & 3 & 3 & 3 & 3 & 3 & 3 & 3 & 2.5 \\
\hline & - & - & - & - & - & - & - & - & - & - & - & - & - & - & 81.9 & 78.6 & 78.6 & 80.6 & 79.4 & 76.6 & 73.1 & 72.8 & 70.8 & 67.4 & 66.3 \\
\hline & o & 1 & 1 & o & o & o & o & o & o & o & o & o & o & o & o & o & o & o & o & o & o & o & o & o & o \\
\hline \multirow[t]{3}{*}{ Cyprus } & 2 & 2 & 1 & 2 & 1.5 & 2 & 1.5 & 1.5 & 1 & 2 & 2 & 2 & 2 & 2.5 & 2 & 2 & 1.5 & 1.5 & 1.5 & 1.5 & 2 & 1 & 2 & 2 & 1 \\
\hline & - & - & - & - & - & - & - & - & - & - & - & - & - & - & 70.5 & 70.2 & 69.7 & 68.9 & 68 & 67.6 & 66.8 & 67 & 67.9 & 66.2 & 64 \\
\hline & o & o & o & o & o & o & o & o & o & o & o & o & 1 & o & o & o & o & o & o & o & o & o & o & o & o \\
\hline \multirow[t]{3}{*}{ Czech Republic } & 1.5 & 1 & 1 & 1.5 & 1 & 1 & 1.5 & 1.5 & 1.5 & 2 & 1.5 & 1.5 & 2 & 2 & 1.5 & 1.5 & 1 & 1 & 1 & 1 & 1 & 1 & 1 & 1 & 1 \\
\hline & - & - & - & - & - & - & - & - & - & - & - & - & - & - & 41.8 & 42.1 & 42.1 & 42.6 & 41.5 & 42.4 & 39.5 & 39.9 & 39.4 & 37.4 & 40.8 \\
\hline & o & o & o & o & o & o & o & o & o & o & o & o & o & o & 1 & o & o & o & o & o & o & o & o & o & o \\
\hline \multirow[t]{3}{*}{ Denmark } & 1 & 1.5 & 1 & 1 & 1 & 1 & 1 & 1 & 1 & 1 & 1.5 & 1 & 1 & 1 & 1 & 1 & 1.5 & 1.5 & 1 & 1 & 1 & 1 & 1 & 1 & 1 \\
\hline & - & - & - & - & - & - & - & - & - & - & - & - & - & - & 24.8 & 22.2 & 21.5 & 23.2 & 22.9 & 23.8 & 23 & 21.9 & 22.8 & 21.4 & 21.5 \\
\hline & o & o & o & o & o & o & o & 1 & o & o & o & o & o & 1 & o & o & o & 1 & o & o & o & o & o & o & o \\
\hline
\end{tabular}




\begin{tabular}{|c|c|c|c|c|c|c|c|c|c|c|c|c|c|c|c|c|c|c|c|c|c|c|c|c|c|}
\hline & 1992 & 1993 & 1994 & 1995 & 1996 & 1997 & 1998 & 1999 & $\begin{array}{c}200 \\
0\end{array}$ & $\begin{array}{c}200 \\
1\end{array}$ & $\begin{array}{c}200 \\
2\end{array}$ & $\begin{array}{c}200 \\
3\end{array}$ & $\begin{array}{c}200 \\
4\end{array}$ & $\begin{array}{c}200 \\
5\end{array}$ & $\begin{array}{c}200 \\
6\end{array}$ & $\begin{array}{c}200 \\
7\end{array}$ & $\begin{array}{c}200 \\
8\end{array}$ & $\begin{array}{c}200 \\
9\end{array}$ & $\begin{array}{c}201 \\
\mathbf{0}\end{array}$ & 2011 & 2012 & 2013 & 2014 & 2015 & 2016 \\
\hline \multirow[t]{3}{*}{ Djibouti } & 3 & 4 & $3 \cdot 5$ & 2 & 2 & 2 & 3.5 & 3 & 2 & 3 & 2 & 2 & 3 & 3 & 2 & - & 2 & 1.5 & 2 & 3 & 3 & 3 & 3 & 3 & 3 \\
\hline & - & - & - & - & - & - & - & - & - & - & - & - & - & - & - & 80.3 & 80 & 80.6 & 81.9 & 82.6 & 83.8 & 85.5 & 87.1 & 88 & 89.7 \\
\hline & o & o & o & o & o & o & o & o & 1 & o & o & o & 0 & o & o & o & 0 & 0 & o & 0 & o & o & 0 & o & o \\
\hline \multirow{3}{*}{$\begin{array}{l}\text { Dominican } \\
\text { Republic }\end{array}$} & 2 & 2 & 2 & 2 & 3 & 3 & 3 & 3 & 2.5 & 3 & 2.5 & 3 & 2.5 & 3 & 3 & 3 & 4 & 4 & 3.5 & 3.5 & 3 & 3 & 3 & 2.5 & 3 \\
\hline & - & - & - & - & - & - & - & - & - & - & - & - & - & - & 85 & 80.6 & 78.4 & 77.7 & 76.8 & 76.9 & 74.1 & 73.2 & 73.4 & 71.2 & 70.8 \\
\hline & o & o & o & o & o & o & o & o & o & o & o & o & o & o & o & o & o & o & o & o & o & o & o & o & o \\
\hline \multirow[t]{3}{*}{ Ecuador } & 3 & 2.5 & 2.5 & 2.5 & 2 & 2.5 & 3 & 3 & 3 & 3 & 3 & 3 & 3 & 3 & 3 & 3 & 2.5 & 2.5 & 2 & 2.5 & 2.5 & 2 & 2 & 2.5 & 2.5 \\
\hline & - & - & - & - & - & - & - & - & - & - & - & - & - & - & 81.2 & 79.9 & 80.3 & 81.2 & 81.7 & 82.2 & 80.1 & 78.6 & $77 \cdot 3$ & 75.9 & 75.6 \\
\hline & 1 & o & 2 & o & o & o & 1 & o & 2 & 3 & 1 & o & 1 & o & o & o & o & o & o & o & o & o & o & o & o \\
\hline \multirow[t]{3}{*}{ Egypt } & 3 & 4 & 4 & 4 & 3.5 & 4 & 3 & 3 & 3 & 3.5 & 3.5 & 3.5 & 3.5 & 3.5 & 4 & 3.5 & 3.5 & 3.5 & 4 & 4 & 3 & 4 & 4 & 4 & 4 \\
\hline & - & - & - & - & - & - & - & - & - & - & - & - & - & - & 89.5 & 89.2 & 88.7 & 89 & 87.6 & 86.8 & 90.4 & 90.6 & 91 & 89.9 & 90.2 \\
\hline & o & 1 & o & 2 & o & 1 & o & o & 1 & o & 1 & o & 5 & 3 & 1 & 1 & 1 & 1 & o & 3 & 5 & 6 & 5 & o & 1 \\
\hline \multirow[t]{3}{*}{ El Salvador } & 4 & 3.5 & 3 & 2.5 & 2.5 & 2.5 & 2 & 3 & 3 & 2 & 1.5 & 3 & 2.5 & 2.5 & 3 & 3 & 2.5 & 2.5 & 2.5 & 2.5 & 2 & 3 & 2 & 2 & 2.5 \\
\hline & - & - & - & - & - & - & - & - & - & - & - & - & - & - & 76.1 & 74.9 & 75.5 & 77.2 & 78.1 & 76 & 74.4 & 73.2 & 72 & 71.4 & 72.5 \\
\hline & o & o & o & o & o & o & o & o & o & o & o & o & 1 & o & o & o & o & o & o & o & o & o & o & o & o \\
\hline \multirow{3}{*}{$\begin{array}{l}\text { Equatorial } \\
\text { Guinea }\end{array}$} & 3 & 3 & 3.5 & 3.5 & 3 & 3 & 3 & 3 & 2.5 & 2.5 & 3 & 2.5 & 3 & 3 & 3 & 3 & 3 & 3 & 3.5 & 2.5 & 3 & 3 & 2.5 & 3 & 2.5 \\
\hline & - & - & - & - & - & - & - & - & - & - & - & - & - & - & 84 & 88.2 & 88 & 88.3 & 88.5 & 88.1 & 86.3 & 86.1 & 85.3 & 84.8 & 85.2 \\
\hline & o & o & o & 0 & o & o & 0 & o & o & o & o & o & o & o & o & 0 & o & o & o & o & o & o & o & o & o \\
\hline \multirow[t]{3}{*}{ Eritrea } & - & 1.5 & 1.5 & 1.5 & 1.5 & 2.5 & 4.5 & 3.5 & 3 & 3 & 2.5 & 3 & 3.5 & 3 & 4 & 4 & 3.5 & 4 & 4 & 4 & 4.5 & 4.5 & 4.5 & 4.5 & 4.5 \\
\hline & - & - & - & - & - & - & - & - & - & - & - & - & - & - & 83.9 & 85.5 & 87.4 & 90.3 & $93 \cdot 3$ & 93.6 & 94.5 & 95 & 95.5 & 96.9 & 98.6 \\
\hline & o & 1 & o & o & o & o & 1 & 1 & 1 & o & o & 1 & 1 & 1 & o & 1 & o & o & o & o & o & o & o & o & o \\
\hline \multirow[t]{3}{*}{ Estonia } & 2 & 2 & 1.5 & 1 & 1.5 & 2 & 1 & 1 & 1 & 1 & 2 & 1 & 1.5 & 1.5 & 1 & 2 & 1.5 & 1 & 1 & 1 & 1 & 1 & 1 & 1 & 1 \\
\hline & - & - & - & - & - & - & - & - & - & - & - & - & - & - & 51 & 50.5 & 51 & 51.2 & 50.7 & 49.3 & 47.5 & $45 \cdot 3$ & 45.2 & 43.7 & 43.4 \\
\hline & o & 1 & o & 0 & o & o & 1 & o & 1 & o & o & o & o & o & o & o & o & o & o & o & o & o & o & o & o \\
\hline \multirow[t]{3}{*}{ Ethiopia } & 3.5 & 3.5 & 4 & 3.5 & 3 & 3.5 & 4.5 & 4.5 & 3.5 & 3.5 & 4 & 4 & 4 & 4 & 3.5 & 4 & 3.5 & 3.5 & 3.5 & 3.5 & 3.5 & 3 & 3.5 & 3.5 & 4.5 \\
\hline & - & - & - & - & - & - & - & - & - & - & - & - & - & - & 91.9 & $95 \cdot 3$ & 96.1 & 98.9 & 98.8 & 98.2 & 97.9 & 98.9 & 97.9 & 97.5 & 97.2 \\
\hline & 1 & o & o & o & o & o & 1 & 1 & 1 & 1 & o & o & 1 & o & 2 & o & 2 & o & o & 1 & o & o & o & o & o \\
\hline
\end{tabular}




\begin{tabular}{|c|c|c|c|c|c|c|c|c|c|c|c|c|c|c|c|c|c|c|c|c|c|c|c|c|c|}
\hline & 1992 & 1993 & 1994 & 1995 & 1996 & 1997 & 1998 & 1999 & $\begin{array}{c}200 \\
\mathbf{0}\end{array}$ & $\begin{array}{c}200 \\
1\end{array}$ & $\begin{array}{c}200 \\
2\end{array}$ & $\begin{array}{c}\mathbf{2 0 0} \\
3\end{array}$ & $\begin{array}{c}200 \\
4\end{array}$ & $\begin{array}{c}200 \\
5\end{array}$ & $\begin{array}{c}200 \\
6\end{array}$ & $\begin{array}{c}\mathbf{2 0 0} \\
7\end{array}$ & $\begin{array}{c}200 \\
8\end{array}$ & $\begin{array}{c}200 \\
9\end{array}$ & $\begin{array}{c}201 \\
\mathbf{0}\end{array}$ & 2011 & 2012 & 2013 & 2014 & 2015 & 2016 \\
\hline \multirow[t]{3}{*}{ Fiji } & 2 & 1 & 1 & 1 & 1 & 1 & 1 & 1 & 4 & 2.5 & 2 & 2 & 2 & 1.5 & 2 & 2.5 & 1.5 & 2 & 2 & 2 & 2 & 2 & 1.5 & 1.5 & 1 \\
\hline & - & - & - & - & - & - & - & - & - & - & - & - & - & - & - & 75.7 & 76.6 & 78.8 & 80.5 & 81.1 & 80.5 & 80.8 & 79 & 76.7 & 76.2 \\
\hline & o & o & o & o & o & o & o & o & o & o & o & o & o & o & o & o & o & o & o & o & o & o & o & o & o \\
\hline \multirow[t]{3}{*}{ Finland } & 1 & 1 & 1 & 1 & 1 & 1 & 1 & 1 & 1 & 1 & 1 & 1 & 1 & 1 & 1 & 1 & 1 & 1 & 1 & 1 & 1 & 1 & 1 & 1 & 1 \\
\hline & - & - & - & - & - & - & - & - & - & - & - & - & - & - & 18.2 & 18.5 & 18.4 & 19.2 & 19.3 & 19.7 & 20 & 18 & 18.7 & 17.7 & 18.8 \\
\hline & o & o & o & o & o & o & o & o & o & o & o & o & o & o & o & o & o & 2 & o & o & o & o & o & o & o \\
\hline \multirow[t]{3}{*}{ France } & 1 & 1 & 1.5 & 2 & 2 & 1 & 1.5 & 2 & 1.5 & 2 & 2 & 2 & 2 & 2 & 2 & 1.5 & 1.5 & 1.5 & 1.5 & 1.5 & 1.5 & 1 & 1.5 & 1.5 & $\begin{array}{l}1.5 \\
\end{array}$ \\
\hline & - & - & - & - & - & - & - & - & - & - & - & - & - & - & $34 \cdot 3$ & 34.1 & 34.8 & 35.3 & 34.9 & 34 & 33.6 & 32.6 & 34.8 & 33.7 & 34.5 \\
\hline & 4 & 1 & 1 & 1 & o & o & 4 & 5 & 1 & 1 & 4 & o & 2 & 1 & 2 & o & 1 & 1 & o & o & 1 & o & o & o & o \\
\hline \multirow[t]{3}{*}{ Gabon } & 2 & 2 & - & 2 & 2 & 2 & 2 & 2 & 2 & 2 & 2 & 2 & 2 & 3 & 3 & 3 & 3 & 3 & 2 & 2 & 2 & 2 & 2 & 2 & 3 \\
\hline & - & - & - & - & - & - & - & - & - & - & - & - & - & - & 73.6 & 73.3 & 75 & 74.4 & 75.3 & 75.3 & 74.6 & 72.9 & 72.2 & 71.2 & 72 \\
\hline & o & o & o & o & o & o & o & o & o & o & o & o & o & o & o & o & o & o & o & o & o & o & o & o & o \\
\hline \multirow[t]{3}{*}{ Gambia } & 1 & 1 & 2 & $\begin{array}{l}2.5 \\
\end{array}$ & 2 & 2 & 2 & 2 & 3 & $\begin{array}{ll}2.5 \\
\end{array}$ & 2 & 2 & 2 & 2 & 3 & 3 & 2.5 & 3 & 3 & 3 & 3 & 3 & 3 & $\begin{array}{ll}3.5 \\
\end{array}$ & 2.5 \\
\hline & - & - & - & - & - & - & - & - & - & - & - & - & - & - & 74 & 76 & 76.9 & 79 & 80.2 & 80.9 & 80.6 & 81.8 & 83.1 & 85.4 & 86.8 \\
\hline & o & o & o & o & o & o & o & o & o & o & o & o & o & o & o & o & o & o & o & o & o & o & o & o & o \\
\hline \multirow[t]{3}{*}{ Georgia } & 3 & 4.5 & 3 & 3 & 2.5 & 2.5 & 3.5 & 2.5 & 2.5 & 3 & 3 & 3 & 3 & 3 & 3 & 3 & 3.5 & 2.5 & 2.5 & 2.5 & 2.5 & 1.5 & 2 & 2 & 2 \\
\hline & - & - & - & - & - & - & - & - & - & - & - & - & - & - & 82.2 & 82.3 & 83.8 & 91.8 & 90.4 & 86.4 & 84.8 & 84.2 & 82.7 & 79.2 & 78.9 \\
\hline & o & o & o & o & 1 & 1 & 2 & o & o & o & o & o & o & o & o & o & o & o & o & 0 & o & o & 1 & o & 0 \\
\hline \multirow[t]{3}{*}{ Germany } & 1.5 & 1 & 2 & 2 & 1 & 1 & 1 & 1.5 & 1 & 2 & 1.5 & 2 & 2 & 1.5 & 1 & 1 & 1 & 1 & 1 & 1 & 1 & 1 & 1 & 1 & 1 \\
\hline & - & - & - & - & - & - & - & - & - & - & - & - & - & - & 39.7 & 38.4 & 37.3 & 36.2 & 35.4 & 33.9 & 31.7 & 29.7 & 30.6 & 28.1 & 28.6 \\
\hline & 1 & o & 1 & 3 & 2 & o & 2 & 2 & o & 3 & 1 & 2 & o & o & 1 & o & o & 4 & o & 2 & o & 2 & 2 & 1 & o \\
\hline \multirow[t]{3}{*}{ Ghana } & 2 & 1.5 & 3 & 2 & 1.5 & 2 & 2 & 2.5 & 2 & 2.5 & 3 & 2.5 & 2.5 & 2 & 2.5 & 2.5 & 2.5 & 2.5 & 3 & 3 & 2.5 & 2 & 2 & 2.5 & 2.5 \\
\hline & - & - & - & - & - & - & - & - & - & - & - & - & - & - & 60.5 & 61.9 & 64.6 & 66.2 & 67.1 & 67.7 & 67.5 & 69.1 & 70.7 & 71.8 & 71.2 \\
\hline & 2 & o & o & o & o & o & o & o & o & o & o & o & o & o & o & o & o & o & o & o & o & o & o & o & o \\
\hline \multirow[t]{3}{*}{ Greece } & 2 & 2 & 2.5 & 2 & 2 & 2 & 2 & 2 & 1.5 & 2 & 2 & 2 & 2 & 2 & 2 & 2.5 & 2.5 & 2.5 & 2 & 2.5 & 2 & 1 & 2.5 & 2.5 & 2.5 \\
\hline & - & - & - & - & - & - & - & - & - & - & - & - & - & - & 41.1 & 43.5 & 45.4 & 46.1 & 45.9 & 47.4 & 50.4 & 50.6 & 52.1 & 52.6 & 55.9 \\
\hline & 2 & 1 & 1 & 2 & 7 & 2 & 9 & 8 & 7 & 2 & 7 & 15 & 7 & 2 & 5 & 1 & 4 & 7 & o & o & 5 & 3 & 1 & o & o \\
\hline
\end{tabular}




\begin{tabular}{|c|c|c|c|c|c|c|c|c|c|c|c|c|c|c|c|c|c|c|c|c|c|c|c|c|c|}
\hline & 1992 & 1993 & 1994 & 1995 & 1996 & 1997 & 1998 & 1999 & $\begin{array}{c}200 \\
\mathbf{0}\end{array}$ & $\begin{array}{c}200 \\
1\end{array}$ & $\begin{array}{c}200 \\
2\end{array}$ & $\begin{array}{c}200 \\
3\end{array}$ & $\begin{array}{c}200 \\
4\end{array}$ & $\begin{array}{c}200 \\
5\end{array}$ & $\underset{6}{200}$ & $\begin{array}{c}200 \\
7\end{array}$ & $\begin{array}{c}200 \\
8\end{array}$ & $\begin{array}{c}200 \\
9\end{array}$ & $\begin{array}{c}201 \\
\mathbf{0}\end{array}$ & 2011 & 2012 & 2013 & 2014 & 2015 & 2016 \\
\hline \multirow[t]{3}{*}{ Grenada } & 1 & 1 & 1 & 1 & 1 & 1 & $\overline{1}$ & 1 & $\overline{1}$ & $\overline{1}$ & 1 & 1 & 1 & $\overline{1}$ & $\overline{1}$ & 1 & 1 & 1 & $\overline{1}$ & 2 & 1 & 1 & 1 & 1 & 1 \\
\hline & - & - & - & - & - & - & - & - & - & - & - & - & - & - & - & 71.6 & 69 & 67.9 & 67 & 66.4 & 65 & 64.6 & 65.2 & 63.5 & 63 \\
\hline & o & o & o & o & o & o & o & o & o & o & o & o & o & o & o & o & o & o & o & o & o & o & o & o & o \\
\hline \multirow[t]{3}{*}{ Guatemala } & 4 & 4 & 4.5 & 4.5 & 4 & 3 & 3.5 & 3 & 3 & 3 & 3 & 3 & $\begin{array}{l}2.5 \\
\end{array}$ & $\begin{array}{ll}3.5 \\
\end{array}$ & 4 & 3.5 & 2.5 & 3 & 3 & 2.5 & 2.5 & 2 & $\begin{array}{l}2.5 \\
\end{array}$ & $\begin{array}{l}2.5 \\
\end{array}$ & 2 \\
\hline & - & - & - & - & - & - & - & - & - & - & - & - & - & - & 84.3 & 81.4 & 80.6 & 80.6 & 81.2 & 80.1 & 79.4 & 80.7 & 80.3 & 80.4 & 83.2 \\
\hline & o & o & o & o & o & o & o & o & o & o & o & o & o & o & o & o & o & o & o & o & o & o & o & o & o \\
\hline \multirow[t]{3}{*}{ Guinea } & 3 & 3 & 3 & 3 & 3 & 2.5 & 3 & 3 & 3.5 & 4 & 2.5 & 2 & 2 & 2.5 & 3 & 4 & 3.5 & 5 & 3 & 3 & 3 & 3 & 3 & $\begin{array}{l}2.5 \\
\end{array}$ & 2.5 \\
\hline & - & - & - & - & - & - & - & - & - & - & - & - & - & - & 99 & 101.3 & 101.8 & 104.6 & 105 & 102.5 & 101.9 & 101.3 & 102.7 & 104.9 & 103.8 \\
\hline & o & o & o & o & o & o & o & o & 1 & o & o & o & o & o & o & o & o & o & o & o & o & o & o & o & o \\
\hline \multirow[t]{3}{*}{ Guinea-Bissau } & 2 & 2.5 & 2 & 2 & 2.5 & 3 & 4.5 & 4 & 2 & 2 & 2 & 2 & 2 & 2.5 & 2.5 & 1.5 & 1.5 & 3.5 & 2.5 & 2 & 3 & 2 & 2 & 2.5 & 2 \\
\hline & - & - & - & - & - & - & - & - & - & - & - & - & - & - & 85.4 & 88.8 & 91.3 & 94.8 & 97.2 & 98.3 & 99.2 & 101.1 & 100. & 99.9 & 99.8 \\
\hline & o & o & o & o & o & o & o & o & o & o & o & o & o & o & o & o & o & o & o & o & o & o & & o & o \\
\hline \multirow[t]{3}{*}{ Guyana } & 2 & 2 & 2 & 1.5 & 2 & 2 & 2 & 2 & 2.5 & 2.5 & 2 & 3 & 3 & 2.5 & 2.5 & 2 & 2 & 3 & 1.5 & 2 & 2 & 2 & 2 & 2 & 2 \\
\hline & - & - & - & - & - & - & - & - & - & - & - & - & - & - & - & 73.3 & 72.2 & 73 & 73 & 72.6 & 71.4 & 70.8 & 71 & 70.5 & 70.9 \\
\hline & o & o & o & o & o & o & o & o & o & o & o & o & o & o & o & o & o & o & o & o & o & o & o & o & o \\
\hline \multirow[t]{3}{*}{ Haiti } & 4 & 4 & 4.5 & 2.5 & 3 & 2.5 & 3 & 3 & 3.5 & 3 & 3 & 3 & 4.5 & 4 & $\begin{array}{ll}3.5 \\
\end{array}$ & $\begin{array}{l}3.5 \\
\end{array}$ & 2.5 & 2.5 & 2.5 & 2 & 3 & 2.5 & 2.5 & 2 & 2.5 \\
\hline & - & - & - & - & - & - & - & - & - & - & - & - & - & - & 104.6 & 100. & 99.3 & 101.8 & 101.6 & 108 & 104.9 & 105.8 & 104.3 & 104.5 & 105.1 \\
\hline & o & o & o & 1 & o & o & o & 0 & o & o & 2 & 1 & o & 0 & o & 9 & o & o & o & o & o & o & o & o & o \\
\hline \multirow[t]{3}{*}{ Honduras } & 3 & 3 & 2.5 & 2.5 & 2.5 & 2 & 2.5 & 3 & 3 & 2.5 & 3 & 3.5 & 3 & 3 & 3 & 3 & 2.5 & 4 & 3 & 2.5 & 2.5 & 3 & 3 & 2.5 & 3.5 \\
\hline & - & - & - & - & - & - & - & - & - & - & - & - & - & - & 76.7 & 74.8 & 74.9 & 77.2 & 80 & 78.3 & 78.5 & 78.3 & 77.9 & 78.1 & 79.8 \\
\hline & o & 1 & o & 1 & o & 1 & o & o & o & o & o & o & o & o & o & o & o & o & o & o & o & 0 & 0 & 0 & 0 \\
\hline \multirow[t]{3}{*}{ Hungary } & 1.5 & 1.5 & 1 & 1.5 & 1 & 1 & 2 & 2 & 2 & 2 & 2 & 2 & 2 & 2 & 2 & 2 & 1.5 & 1.5 & 1.5 & 1.5 & 1.5 & 1.5 & 1.5 & 1.5 & 2 \\
\hline & - & - & - & - & - & - & - & - & - & - & - & - & - & - & 46.7 & 51.2 & 50.9 & 50.7 & 50.1 & 48.7 & 48.3 & 47.6 & 48.3 & 49.1 & 52.7 \\
\hline & 1 & o & o & o & o & o & o & o & o & o & o & o & o & o & o & o & o & o & o & o & o & o & o & o & o \\
\hline \multirow[t]{3}{*}{ Iceland } & 1 & 1 & 1 & 1 & 1 & 1 & 1 & 1 & 1 & 1 & 1 & 1 & 1 & 1 & 1 & 1 & 1 & 1 & 1 & 1 & 1 & 1 & 1 & 1 & 1 \\
\hline & - & - & - & - & - & - & - & - & - & - & - & - & - & - & - & 21.1 & 20.9 & 29 & 29.8 & 30.1 & 29.1 & 24.7 & 25.9 & 23.4 & 22.8 \\
\hline & o & o & o & o & o & o & o & o & o & o & o & o & o & o & o & o & o & o & o & o & o & o & o & o & o \\
\hline
\end{tabular}




\begin{tabular}{|c|c|c|c|c|c|c|c|c|c|c|c|c|c|c|c|c|c|c|c|c|c|c|c|c|c|}
\hline & 1992 & 1993 & 1994 & 1995 & 1996 & 1997 & 1998 & 1999 & $\begin{array}{c}200 \\
\text { o }\end{array}$ & $\begin{array}{c}200 \\
1\end{array}$ & $\begin{array}{c}200 \\
2\end{array}$ & $\begin{array}{c}200 \\
3\end{array}$ & $\begin{array}{c}200 \\
4\end{array}$ & $\begin{array}{c}200 \\
5\end{array}$ & $\begin{array}{c}200 \\
6\end{array}$ & $\begin{array}{c}200 \\
7\end{array}$ & $\stackrel{200}{8}$ & $\begin{array}{c}200 \\
9\end{array}$ & $\begin{array}{c}201 \\
\mathbf{0}\end{array}$ & 2011 & 2012 & 2013 & 2014 & 2015 & 2016 \\
\hline \multirow[t]{3}{*}{ India } & 4.5 & 5 & 5 & 4.5 & 4.5 & 4 & 4 & 3.5 & 4 & 4 & 4 & 4 & 3.5 & 3.5 & 3.5 & 4 & 4 & 4 & 4 & 4 & 4 & 4 & 3.5 & 3.5 & 3.5 \\
\hline & - & - & - & - & - & - & - & - & - & - & - & - & - & - & 70.4 & 70.8 & 72.9 & 77.8 & 79.2 & 79.3 & 78 & 77.5 & 76.9 & 79.3 & 79.6 \\
\hline & 6 & 3 & 8 & 9 & 12 & 7 & 1 & 7 & 5 & 4 & 8 & 7 & 5 & 5 & 4 & 3 & 6 & 7 & 1 & 5 & o & 2 & 5 & 2 & o \\
\hline \multirow[t]{3}{*}{ Indonesia } & 4 & 4 & 4 & 4 & 3.5 & 3.5 & 4 & 4 & 4 & 4 & 4 & 3.5 & 4 & 3.5 & 3 & 3 & 3 & 3.5 & 3 & 3 & 3 & 3 & 3 & 3 & 3 \\
\hline & - & - & - & - & - & - & - & - & - & - & - & - & - & - & 89.2 & 84.4 & 83.3 & 84.1 & 83.1 & 81.6 & 80.6 & 78.2 & 76.8 & 75 & 74.9 \\
\hline & o & 2 & o & 2 & o & 2 & 4 & 3 & 10 & 1 & o & 1 & o & 2 & o & 1 & 1 & 5 & 3 & o & o & 3 & 3 & 2 & o \\
\hline \multirow[t]{3}{*}{$\begin{array}{l}\text { Iran } \\
\end{array}$} & 4 & 3.5 & 4 & 4.5 & 3.5 & 4 & 3.5 & 4 & 3.5 & 3 & 3.5 & 3 & 3.5 & 3.5 & 4 & 4 & 4 & 4 & 4 & 4 & 4 & 3.5 & 3.5 & 3.5 & 4 \\
\hline & - & - & - & - & - & - & - & - & - & - & - & - & - & - & 84 & 82.8 & 85.7 & 90 & 92.2 & 90.2 & 89.6 & 89.7 & 87.2 & 87.1 & 86.9 \\
\hline & 1 & o & 1 & 2 & o & o & 1 & 3 & 1 & 1 & o & 3 & 3 & 4 & o & 1 & 1 & 1 & o & o & 3 & 5 & 5 & o & o \\
\hline \multirow[t]{3}{*}{ Iraq } & 5 & 5 & 5 & 5 & 5 & 5 & 5 & 5 & 5 & 4.5 & 4.5 & 5 & 5 & 5 & 5 & 5 & 5 & 4.5 & 4.5 & 4 & 4 & 5 & 5 & 5 & 5 \\
\hline & - & - & - & - & - & - & - & - & - & - & - & - & - & - & 109 & 111.4 & 110.6 & 108 & 107.3 & 104. & 104.3 & 103.9 & 102.2 & 104.4 & 104.7 \\
\hline & o & o & 3 & 2 & o & o & 2 & 2 & 1 & 4 & o & 13 & 30 & 20 & 9 & 6 & 2 & 6 & 2 & & 3 & 9 & 12 & o & 1 \\
\hline \multirow[t]{3}{*}{ Ireland } & 1 & 1 & 1 & 1 & 1 & 1 & 1 & 1 & 1 & 1 & 1 & 1 & 1.5 & 1 & 1 & 1.5 & 1 & 1 & 1 & 1 & 1 & 1 & 1 & 1 & 1 \\
\hline & - & - & - & - & - & - & - & - & - & - & - & - & - & - & 18.6 & 19.5 & 19.9 & 21.6 & 22.4 & 25.3 & 26.5 & 24.8 & 26.1 & 24.6 & 22.5 \\
\hline & o & o & o & o & o & 1 & 4 & o & o & 1 & o & o & o & o & o & 1 & o & 2 & o & 1 & o & o & o & o & o \\
\hline \multirow{3}{*}{$\begin{array}{l}\text { Israel and West } \\
\text { Bank }\end{array}$} & 3.5 & 3.5 & 3.5 & 3.5 & 4 & 3.5 & 3 & 3 & 4 & 4.5 & 5 & 4.5 & 4.5 & 4 & 4 & 4 & 5 & 5 & 4 & 4 & 4 & 4 & 4 & 3.5 & 3.5 \\
\hline & - & - & - & - & - & - & - & - & - & - & - & - & - & - & 79.4 & 79.6 & 83.6 & 84.6 & 84.6 & 84.4 & 82.2 & 80.8 & 79.5 & 79.4 & 79.7 \\
\hline & 2 & 3 & o & o & o & o & 1 & 1 & 4 & 2 & 2 & 3 & o & 1 & 4 & o & 3 & 1 & o & 1 & 2 & 1 & 2 & 1 & 1 \\
\hline \multirow[t]{3}{*}{$\begin{array}{l}\text { Italy } \\
\end{array}$} & 1.5 & 2 & 2 & 2 & 1.5 & 1 & 1.5 & 1.5 & 1.5 & 2 & 2 & 1.5 & 1.5 & 2 & 1 & 2 & 2 & 1.5 & 2 & 2 & 1.5 & 2 & 2 & 1.5 & 2 \\
\hline & - & - & - & - & - & - & - & - & - & - & - & - & - & - & 35.1 & 37.1 & 39.9 & 43.9 & 45.7 & 45.8 & 45.8 & 44.6 & 43.4 & 43.2 & 43.1 \\
\hline & 2 & 1 & o & 2 & 1 & o & 2 & 3 & 3 & 3 & 3 & 4 & 1 & 1 & o & 1 & o & 2 & o & o & 1 & o & 2 & o & o \\
\hline \multirow[t]{3}{*}{ Jamaica } & 3 & 2.5 & 2 & 3 & 1.5 & 2 & 3 & 3 & 3 & 3.5 & 3 & 3.5 & 3 & 3 & 3 & 3 & 3.5 & 3.5 & 4 & 3.5 & 3 & 3 & 3 & 3 & 3 \\
\hline & - & - & - & - & - & - & - & - & - & - & - & - & - & - & 66.8 & 65.1 & 65.7 & 68.6 & 67.4 & 67.1 & 65.8 & 65.6 & 64.9 & 64.6 & 65 \\
\hline & o & o & o & o & o & o & o & o & o & o & o & o & o & o & o & o & o & o & o & o & o & o & o & o & o \\
\hline \multirow[t]{3}{*}{ Japan } & 1 & 1 & 1.5 & 1.5 & 2 & 1 & 1 & 1.5 & 1 & 1.5 & 2 & 2 & 1.5 & 1 & 1 & 1 & 1 & 1 & 1.5 & 1 & 1.5 & 1 & 1.5 & 1 & 1 \\
\hline & - & - & - & - & - & - & - & - & - & - & - & - & - & - & 28 & 28.5 & 29.7 & 31.2 & 31.3 & 31 & 43.5 & 36.1 & 36.3 & 36 & 35.1 \\
\hline & 1 & o & o & o & o & o & o & o & 1 & o & 2 & o & o & o & o & o & o & o & o & o & o & o & o & o & o \\
\hline
\end{tabular}




\begin{tabular}{|c|c|c|c|c|c|c|c|c|c|c|c|c|c|c|c|c|c|c|c|c|c|c|c|c|c|}
\hline & 1992 & 1993 & 1994 & 1995 & 1996 & 1997 & 1998 & 1999 & $\begin{array}{c}200 \\
\mathbf{0}\end{array}$ & $\begin{array}{c}200 \\
1\end{array}$ & $\begin{array}{c}200 \\
2\end{array}$ & $\begin{array}{c}200 \\
3\end{array}$ & $\begin{array}{c}200 \\
4\end{array}$ & $\begin{array}{c}200 \\
5\end{array}$ & $\begin{array}{c}200 \\
6\end{array}$ & $\begin{array}{c}\mathbf{2 0 0} \\
7\end{array}$ & $\begin{array}{c}200 \\
8\end{array}$ & $\begin{array}{c}200 \\
9\end{array}$ & $\begin{array}{c}\mathbf{2 0 1} \\
\mathbf{0}\end{array}$ & 2011 & 2012 & 2013 & 2014 & 2015 & 2016 \\
\hline \multirow[t]{3}{*}{ Jordan } & 2 & 2 & 2.5 & 2.5 & 2 & 2 & 2.5 & 2.5 & 2.5 & 2.5 & 2.5 & 2 & 2 & 2.5 & 3 & 3 & 3.5 & 3 & 3.5 & 3 & 3 & 3 & 2.5 & 3 & 2.5 \\
\hline & - & - & - & - & - & - & - & - & - & - & - & - & - & - & 77 & 76.6 & 77.3 & 77.9 & 77 & 74.5 & 74.8 & 75.7 & 76.7 & 76.9 & 78 \\
\hline & 1 & o & o & 1 & o & 1 & o & 1 & o & 1 & o & 1 & 2 & 2 & o & o & o & 1 & o & o & 2 & o & o & o & 1 \\
\hline \multirow[t]{3}{*}{ Kazakhstan } & 1 & 1 & 2 & 1.5 & 2.5 & 2 & 3 & 2.5 & 3 & 3 & 3 & 2 & 2.5 & 2.5 & 2.5 & 2.5 & 2.5 & 2.5 & 3 & 3 & 3 & 3 & 3 & 2.5 & 3 \\
\hline & - & - & - & - & - & - & - & - & - & - & - & - & - & - & 71.9 & 72.3 & 72.4 & 72.5 & 72.7 & 70.2 & 70.9 & 69.8 & 68.5 & 68.3 & 66.5 \\
\hline & o & o & o & 1 & o & o & o & o & 1 & o & 1 & o & o & o & o & o & o & 1 & o & 2 & o & o & o & o & o \\
\hline \multirow[t]{3}{*}{ Kenya } & 4 & 3.5 & 4 & 3.5 & 3.5 & 4 & 3 & 3 & 3 & 3 & 4 & 3.5 & 3 & 3.5 & 3.5 & 4 & 4 & 3.5 & 3.5 & 2.5 & 4 & 3.5 & 3.5 & 3.5 & 3.5 \\
\hline & - & - & - & - & - & - & - & - & - & - & - & - & - & - & 88.6 & 91.3 & 93.4 & 101.4 & 100.7 & 98.7 & 98.4 & 99.6 & 99 & 97.3 & 98.3 \\
\hline & 2 & o & o & 2 & o & 2 & o & 1 & o & o & o & o & 1 & o & o & 1 & o & 1 & o & o & o & o & o & o & 1 \\
\hline \multirow[t]{3}{*}{ Korea, ROK } & 3 & 2 & 2.5 & 2.5 & 2.5 & 2 & 2 & 2 & 2 & 2 & 3 & 2 & 2 & 1.5 & 1.5 & 2 & 2 & 2.5 & 1 & 1 & 1.5 & 1 & 2 & 1.5 & 2 \\
\hline & - & - & - & - & - & - & - & - & - & - & - & - & - & - & 39.9 & 39.7 & 40.6 & 41.6 & 41.3 & 38.8 & 37.6 & 35.4 & 36.4 & 36.3 & 36.1 \\
\hline & o & o & o & o & 1 & o & o & o & o & o & o & o & o & o & o & o & o & o & o & o & o & o & o & o & o \\
\hline \multirow[t]{3}{*}{ Korea, DPRK } & - & - & - & - & - & 5 & 5 & 5 & 5 & 5 & 3.5 & 4 & 4 & 4 & 4 & 4 & 5 & 5 & 5 & 5 & 5 & 5 & 5 & 5 & 5 \\
\hline & - & - & - & - & - & - & - & - & - & - & - & - & - & - & 97.3 & 97.7 & 97.7 & 98.3 & 97.8 & 95.6 & 95.5 & 95.1 & 94 & 93.8 & 93.9 \\
\hline & o & o & o & o & o & o & o & o & o & o & o & o & o & o & o & o & o & o & o & o & o & o & o & o & o \\
\hline \multirow[t]{3}{*}{ Kosovo } & - & - & - & - & - & - & - & - & - & - & - & - & - & - & - & - & 1 & 2 & 1 & 2 & 1 & 1.5 & 2 & 1 & 1.5 \\
\hline & - & - & - & - & - & - & - & - & - & - & - & - & - & - & - & - & - & - & - & - & - & - & - & - & - \\
\hline & 1 & o & o & o & o & o & o & 1 & o & 1 & o & o & o & o & o & o & o & o & o & o & o & o & o & o & o \\
\hline \multirow[t]{3}{*}{ Kuwait } & 3 & 3 & 2.5 & 2.5 & 2 & 2 & 2 & 1.5 & 1.5 & 1.5 & 2 & 2 & 2 & 2 & 2 & 2 & 1.5 & 2 & 2 & 2 & 2 & 2 & 2 & 2.5 & 2 \\
\hline & - & - & - & - & - & - & - & - & - & - & - & - & - & - & 60.8 & 62.1 & 62 & 63.4 & 61.5 & 59.5 & 58.8 & 59.6 & 59 & 57.5 & 58.5 \\
\hline & o & o & o & o & o & o & o & o & o & o & o & o & o & o & o & o & o & o & o & o & o & 1 & o & o & o \\
\hline \multirow[t]{3}{*}{ Kyrgyzstan } & 1 & 1 & 1.5 & 1.5 & 1.5 & 2 & 2 & 3 & 2.5 & 2.5 & 2.5 & 2.5 & 2.5 & 2.5 & 3 & 3 & 1.5 & 2.5 & 4 & 3 & 3 & 3 & 3 & 3 & 3 \\
\hline & - & - & - & - & - & - & - & - & - & - & - & - & - & - & 90.3 & 88.2 & 88.8 & 89.1 & 88.4 & 91.8 & 87.4 & 85.7 & 83.9 & 82.2 & 81.1 \\
\hline & o & 1 & o & 1 & 1 & 2 & o & o & 2 & o & 1 & o & o & o & o & o & o & o & o & 1 & o & o & o & o & o \\
\hline \multirow[t]{3}{*}{ Laos } & 2.5 & 2.5 & 2 & 1.5 & 1 & 2 & 2.5 & 2 & 3 & 2.5 & 2.5 & 3 & 3 & 3 & 3 & 3 & 1.5 & 2 & 2 & 2 & 2 & 2 & 2 & 2 & 2 \\
\hline & - & - & - & - & - & - & - & - & - & - & - & - & - & - & 87.9 & 87.2 & 88.7 & 89 & 88.7 & 86.7 & 85.5 & 83.7 & 84.3 & 84.4 & 84.4 \\
\hline & o & o & o & o & o & o & o & o & 1 & o & o & o & o & o & o & o & o & o & o & o & o & o & o & o & o \\
\hline
\end{tabular}




\begin{tabular}{|c|c|c|c|c|c|c|c|c|c|c|c|c|c|c|c|c|c|c|c|c|c|c|c|c|c|}
\hline & 1992 & 1993 & 1994 & 1995 & 1996 & 1997 & 1998 & 1999 & $\begin{array}{c}200 \\
\mathbf{0}\end{array}$ & $\begin{array}{c}200 \\
1\end{array}$ & $\begin{array}{c}200 \\
2\end{array}$ & $\begin{array}{c}\mathbf{2 0 0} \\
3\end{array}$ & $\begin{array}{c}200 \\
4\end{array}$ & $\begin{array}{c}200 \\
5\end{array}$ & $\begin{array}{c}200 \\
6\end{array}$ & $\begin{array}{c}\mathbf{2 0 0} \\
7\end{array}$ & $\begin{array}{c}200 \\
8\end{array}$ & $\begin{array}{c}200 \\
9\end{array}$ & $\begin{array}{c}201 \\
\mathbf{0}\end{array}$ & 2011 & 2012 & 2013 & 2014 & 2015 & 2016 \\
\hline \multirow[t]{3}{*}{ Latvia } & 1 & 1.5 & 1.5 & 2 & 1.5 & 2 & 2 & 1.5 & 1 & 1.5 & 1 & 1 & 1 & 2 & 1.5 & 1.5 & 2 & 2 & 2 & 2 & 1.5 & 1 & 1.5 & 1.5 & 1 \\
\hline & - & - & - & - & - & - & - & - & - & - & - & - & - & - & 56.2 & 56.7 & 54.5 & 54.6 & 55.4 & 54.2 & 51.9 & 47.9 & 48 & 48.5 & 47.4 \\
\hline & o & 1 & o & o & o & o & 1 & o & 1 & o & o & o & o & o & o & o & o & o & o & o & o & o & o & o & o \\
\hline \multirow[t]{3}{*}{$\begin{array}{l}\text { Lebanon } \\
\end{array}$} & 3.5 & 3.5 & 4 & 4 & 3 & 3.5 & 3 & 3 & 3 & 3 & 3 & 3 & 3 & 3 & 3 & 3.5 & 3 & 2.5 & 2.5 & 2.5 & 3 & 3 & 3 & 2.5 & 2.5 \\
\hline & - & - & - & - & - & - & - & - & - & - & - & - & - & - & 80.5 & 92.4 & 95.7 & 93.5 & 90.9 & 87.7 & 85.8 & 86.3 & 86.9 & 88.1 & 89.6 \\
\hline & o & 3 & 1 & 1 & o & o & o & 1 & o & o & o & o & 2 & 2 & 4 & o & o & 1 & o & 2 & 4 & 4 & 4 & o & o \\
\hline \multirow[t]{3}{*}{ Lesotho } & 3 & 2.5 & 3 & 2 & 1.5 & 2 & 2.5 & 2 & 1.5 & 2 & 2 & 2 & 2 & 2 & 2 & 3 & 2 & 2 & 2 & 2 & 2 & 2 & 2 & 3 & 3 \\
\hline & - & - & - & - & - & - & - & - & - & - & - & - & - & - & - & 81.2 & 81.7 & 81.8 & 82.2 & 80.4 & 79 & 79.4 & 78.6 & 79.9 & 80.9 \\
\hline & o & o & o & o & o & o & o & o & o & o & o & o & o & o & o & o & o & o & o & o & o & o & o & o & o \\
\hline \multirow[t]{3}{*}{ Liberia } & 4.5 & 5 & 5 & 5 & 5 & 3 & 3.5 & 4.5 & 4 & 5 & 4.5 & 4.5 & 3 & 3 & 3 & 3 & 2.5 & 2.5 & 2.5 & 2 & 2 & 2 & 2 & 3 & 2 \\
\hline & - & - & - & - & - & - & - & - & - & - & - & - & - & - & 99 & 92.9 & 91 & 91.8 & 91.7 & 94 & 93.3 & 95.1 & 94.3 & 97.3 & 95.5 \\
\hline & 1 & 1 & 2 & o & o & o & o & 1 & o & o & o & o & o & o & 1 & o & o & o & o & o & o & o & o & o & o \\
\hline \multirow[t]{3}{*}{ Libya } & 3 & 3 & 3 & 3 & 3 & 3.5 & 3.5 & 3.5 & 3 & 3 & 3 & 3 & 3 & 3 & 3 & 3 & 3 & 3 & 3 & 5 & 4 & 4 & 4.5 & 4 & 4.5 \\
\hline & - & - & - & - & - & - & - & - & - & - & - & - & - & - & 68.5 & 69.3 & 70 & 69.4 & 69.1 & 68.7 & 84.9 & 84.5 & 87.8 & 95.3 & 96.4 \\
\hline & o & o & o & 1 & 1 & o & 1 & o & 2 & o & o & o & 1 & o & o & 1 & 1 & o & o & 6 & 8 & 6 & 10 & 2 & 2 \\
\hline \multirow[t]{3}{*}{ Lithuania } & 1 & 1 & 1 & 1 & 1.5 & 1 & 1 & 1 & 1 & 1.5 & 1 & 1 & 1 & 1 & 1.5 & 1 & 1 & 1.5 & 1.5 & 1.5 & 1.5 & 1 & 1.5 & 1 & 1 \\
\hline & - & - & - & - & - & - & - & - & - & - & - & - & - & - & 49.7 & 49 & 48.7 & 48 & 47.8 & 45.3 & 44.2 & 43 & 43.2 & 43 & 42.4 \\
\hline & o & o & o & o & o & o & o & o & o & o & o & o & o & o & o & o & o & o & o & o & o & o & o & o & o \\
\hline \multirow[t]{3}{*}{ Luxembourg } & 1 & 1 & 1 & 1 & 1 & 1 & 1 & 1 & 1 & 1 & 1 & 1 & 1 & 1 & 1 & 1 & 1 & 1 & 1 & 1 & 1 & 1 & 1 & 1 & 1 \\
\hline & - & - & - & - & - & - & - & - & - & - & - & - & - & - & - & 28.1 & 27.9 & 27.6 & 27.3 & 26.1 & 25.5 & 23.3 & 24.6 & 22.1 & 24.1 \\
\hline & o & o & o & o & o & o & o & o & o & o & o & o & o & o & o & o & o & o & o & o & o & o & o & o & o \\
\hline \multirow[t]{3}{*}{ Macedonia } & 2 & 1.5 & 1.5 & 1 & 1 & 2.5 & 2.5 & 2 & 2 & 4 & 3 & 2.5 & 2 & 2 & 2 & 2.5 & 2 & 2 & 2 & 2 & 2 & 2 & 2 & 2.5 & 2 \\
\hline & - & - & - & - & - & - & - & - & - & - & - & - & - & - & 75.1 & 74.1 & 74.6 & 74.4 & 72.7 & 71 & 69.1 & 68 & 66.4 & 64.5 & 67 \\
\hline & 1 & o & o & o & o & o & o & 2 & o & 2 & o & o & o & o & o & o & o & o & o & o & o & o & o & o & o \\
\hline \multirow[t]{3}{*}{ Madagascar } & 3 & 3 & 2 & 2 & 2 & 2 & 2 & 2 & 2 & 2 & 3 & 2 & 2 & 2 & 2 & 2 & 2 & 4 & 2.5 & 3 & 3.5 & 3 & 3 & 3 & 3 \\
\hline & - & - & - & - & - & - & - & - & - & - & - & - & - & - & - & 76.5 & 76.7 & 81.6 & 82.6 & 83.2 & 82.5 & 82.7 & 83.1 & 83.6 & 84.2 \\
\hline & 1 & o & o & o & o & o & o & o & o & o & o & o & o & o & o & o & o & o & o & o & o & 1 & o & o & o \\
\hline
\end{tabular}




\begin{tabular}{|c|c|c|c|c|c|c|c|c|c|c|c|c|c|c|c|c|c|c|c|c|c|c|c|c|c|}
\hline & 1992 & 1993 & 1994 & 1995 & 1996 & 1997 & 1998 & 1999 & $\begin{array}{c}200 \\
\mathbf{0}\end{array}$ & $\begin{array}{c}200 \\
1\end{array}$ & $\begin{array}{c}200 \\
2\end{array}$ & $\begin{array}{c}200 \\
3\end{array}$ & $\begin{array}{c}200 \\
4\end{array}$ & $\begin{array}{c}200 \\
5\end{array}$ & $\begin{array}{c}200 \\
6\end{array}$ & $\begin{array}{c}200 \\
7\end{array}$ & $\begin{array}{c}200 \\
8\end{array}$ & $\begin{array}{c}200 \\
9\end{array}$ & $\begin{array}{c}201 \\
\mathbf{0}\end{array}$ & 2011 & 2012 & 2013 & 2014 & 2015 & 2016 \\
\hline \multirow[t]{3}{*}{ Malawi } & $\overline{3}$ & 2 & 2 & 2 & 2 & 2 & 2 & 2 & 2 & 2 & 3 & 2.5 & 2.5 & 3 & 3 & 2.5 & 2.5 & 2 & 2.5 & 3 & 2.5 & 2 & 2.5 & 2 & 2.5 \\
\hline & - & - & - & - & - & - & - & - & - & - & - & - & - & - & 89.8 & 92.2 & 92.9 & 93.8 & 93.6 & 91.2 & 88.8 & 89.2 & 89.1 & 86.9 & 87.6 \\
\hline & o & o & o & o & o & o & o & 0 & o & 0 & o & o & 0 & o & 0 & 0 & 0 & 0 & o & 0 & o & o & 0 & o & 0 \\
\hline \multirow[t]{3}{*}{ Malaysia } & 2 & 1.5 & 2 & 2.5 & 2 & 2 & 3 & 2 & 2 & 2 & 2.5 & 2.5 & 2.5 & 2.5 & 2.5 & 2.5 & 2 & 2 & 2 & 2.5 & 2.5 & 2.5 & 3 & 3 & 2.5 \\
\hline & - & - & - & - & - & - & - & - & - & - & - & - & - & 1 & 66.1 & 65.9 & 67.2 & 68.9 & 69.2 & 68.7 & 68.5 & 66.1 & 66.2 & 65.9 & 66.1 \\
\hline & o & o & o & 3 & o & o & o & o & 2 & 1 & o & o & o & o & o & o & o & 1 & o & o & o & 1 & 2 & o & o \\
\hline \multirow[t]{3}{*}{ Maldives } & 2 & 2 & 1.5 & 2 & 2 & 1.5 & 1.5 & 1.5 & 1.5 & 2 & 2 & 2 & 2 & 2 & 2.5 & 2 & 1.5 & 1 & 1.5 & 1.5 & 2.5 & 1 & 2 & 2.5 & 2.5 \\
\hline & - & - & - & - & - & - & - & - & - & - & - & - & - & - & - & 81.1 & 80.4 & 78.8 & 78.3 & 75.6 & 75.1 & 75.4 & 75.4 & 74.3 & 74 \\
\hline & o & o & o & o & o & o & o & o & o & o & o & o & o & o & o & o & o & o & o & 1 & o & o & 1 & o & o \\
\hline \multirow[t]{3}{*}{ Mali } & 3 & 2 & 3 & 2 & 1 & 2 & 2 & 2 & 1.5 & 2 & 2 & 2 & 2 & 2 & 2 & 2 & 2 & 1.5 & 1.5 & 1.5 & 3.5 & 4 & 3.5 & 3.5 & 3.5 \\
\hline & - & - & - & - & - & - & - & - & - & - & - & - & - & - & 74.6 & 75.5 & 75.6 & 78.7 & 79.3 & 79.3 & 77.9 & 89.3 & 89.8 & 92.9 & 95.2 \\
\hline & 1 & o & 2 & o & o & o & 1 & o & o & o & o & o & 1 & o & 1 & 1 & 1 & o & o & 3 & 5 & 4 & o & 2 & 1 \\
\hline \multirow[t]{3}{*}{ Malta } & 1 & 1 & 1 & 1 & 1 & 1 & 1 & 1 & 1 & 1 & 1 & 1 & 1.5 & 1.5 & 1.5 & 1.5 & 1.5 & 1 & 1 & 1 & 1 & 1 & 1 & 1 & 1 \\
\hline & - & - & - & - & - & - & - & - & - & - & - & - & - & - & - & 48.5 & 48.3 & 48.8 & 48.2 & $45 \cdot 4$ & 43.8 & 42.4 & 43 & 40.9 & 39.6 \\
\hline & o & o & o & o & o & o & o & o & 1 & o & o & o & o & o & o & o & o & o & o & o & o & o & o & o & o \\
\hline \multirow[t]{3}{*}{ Mauritania } & 3.5 & 2.5 & 2 & 2 & 1.5 & 2.5 & 2 & 2 & 2.5 & 2.5 & 3 & 2.5 & 3 & 3 & 3 & 2.5 & 3 & 3 & 2.5 & 2.5 & 2.5 & 2 & 2.5 & 2.5 & 3 \\
\hline & - & - & - & - & - & - & - & - & - & - & - & - & - & - & 87.8 & 86.7 & 86.1 & 88.7 & 89.1 & 88 & 87.6 & 91.7 & 93 & 94.9 & 95.4 \\
\hline & o & o & o & o & o & o & 1 & o & o & o & o & 0 & o & 1 & 0 & 0 & 0 & o & 0 & 1 & 2 & 1 & 0 & 0 & 0 \\
\hline \multirow[t]{3}{*}{ Mauritius } & 1 & 1 & 2 & 1 & 1 & 1 & 2 & 2 & 2 & 2 & 1 & 1 & 2 & 2 & 2 & 2 & 2 & 2 & 2 & 2 & 2 & 2 & 2 & 2 & 2 \\
\hline & - & - & - & - & - & - & - & - & - & - & - & - & - & - & 41.9 & 42.7 & 42.4 & 44.7 & 44.4 & 44.2 & 44.7 & 44.5 & 46.1 & 45.2 & 43.2 \\
\hline & o & o & o & o & o & o & o & o & o & o & o & o & o & o & o & o & o & o & o & o & o & o & o & o & o \\
\hline \multirow[t]{3}{*}{ Mexico } & 3 & 3.5 & 4 & 4 & 3.5 & 3.5 & 3 & 3 & 3 & 3 & 3 & 3 & 3 & 3 & 3.5 & 3 & 3.5 & 4 & 4 & 4 & 4 & 3.5 & 4 & 4 & 3 \\
\hline & - & - & - & - & - & - & - & - & - & - & - & - & - & - & 73.1 & 72.6 & 72.2 & 75.4 & 76.1 & 75.1 & 73.6 & 73.1 & 71.1 & 71.9 & 70.4 \\
\hline & 2 & o & o & 1 & 3 & o & o & o & 2 & 1 & o & 1 & 1 & o & o & 1 & o & o & o & 2 & o & o & o & o & o \\
\hline \multirow[t]{3}{*}{ Moldova } & 3.5 & 3 & 2 & 1.5 & 2 & 2 & 1.5 & 2.5 & 2 & 2 & 2 & 2.5 & 3 & 2.5 & 2.5 & 2.5 & 2.5 & 3 & 3 & 2.5 & 2.5 & 2 & 2.5 & 2 & 2 \\
\hline & - & - & - & - & - & - & - & - & - & - & - & - & - & - & 82.5 & 85.7 & 85.7 & 85.1 & 83.8 & 81.2 & 78.7 & 76.5 & 75.1 & 73 & 73.2 \\
\hline & o & 1 & o & o & o & o & o & o & o & o & o & o & o & o & o & o & o & o & o & o & o & o & o & o & o \\
\hline
\end{tabular}




\begin{tabular}{|c|c|c|c|c|c|c|c|c|c|c|c|c|c|c|c|c|c|c|c|c|c|c|c|c|c|}
\hline & 1992 & 1993 & 1994 & 1995 & 1996 & 1997 & 1998 & 1999 & $\begin{array}{c}200 \\
\mathbf{0}\end{array}$ & $\begin{array}{c}200 \\
1\end{array}$ & $\begin{array}{c}200 \\
2\end{array}$ & $\begin{array}{c}200 \\
3\end{array}$ & $\begin{array}{c}200 \\
4\end{array}$ & $\begin{array}{c}200 \\
5\end{array}$ & $\begin{array}{c}200 \\
6\end{array}$ & $\begin{array}{c}\mathbf{2 0 0} \\
7\end{array}$ & $\begin{array}{c}200 \\
8\end{array}$ & $\begin{array}{c}200 \\
9\end{array}$ & $\begin{array}{c}201 \\
\mathbf{0}\end{array}$ & 2011 & 2012 & 2013 & 2014 & 2015 & 2016 \\
\hline \multirow[t]{3}{*}{ Mongolia } & 1 & 1 & 1 & 1.5 & 1.5 & 1 & 2 & 1 & 1 & 1 & 2 & 2.5 & 2.5 & 2.5 & 2.5 & 2.5 & 3 & 2.5 & 2.5 & 2.5 & 1.5 & 2 & 2.5 & 2.5 & 2.5 \\
\hline & - & - & - & - & - & - & - & - & - & - & - & - & - & - & 58.4 & 58.4 & 57.8 & 61.9 & 60.1 & 59.6 & 58.7 & 57.8 & 58.1 & 57 & 56.6 \\
\hline & o & o & o & o & o & o & 1 & o & o & o & o & o & o & o & o & o & o & o & o & o & o & o & o & o & o \\
\hline \multirow[t]{3}{*}{ Montenegro } & - & - & - & - & - & - & - & - & - & - & - & - & - & - & - & - & 2 & 2 & 1.5 & 1.5 & 1.5 & 2 & 2 & 2 & 1 \\
\hline & - & - & - & - & - & - & - & - & - & - & - & - & - & - & - & 55.6 & 55.8 & 58 & 57.3 & 56.3 & 55.5 & 54.4 & 55.7 & 54.2 & 55.2 \\
\hline & o & o & o & o & o & o & o & o & o & o & o & o & o & o & o & o & 1 & o & o & o & o & o & o & o & o \\
\hline \multirow[t]{3}{*}{ Morocco } & 3 & 3 & 3 & 3 & 2.5 & 2.5 & 2 & 2 & 2.5 & 2 & 2 & 2.5 & 2.5 & 3 & 3 & 3 & 3 & 3 & 3 & 3 & 3 & 2.5 & 2.5 & 2.5 & 2.5 \\
\hline & - & - & - & - & - & - & - & - & - & - & - & - & - & - & 76.5 & 76 & 75.8 & 77.1 & 77 & 76.3 & 76.1 & 74.3 & 74.4 & 74.6 & 74.2 \\
\hline & o & o & 1 & o & 1 & o & 1 & o & o & o & o & 1 & 0 & 1 & 1 & 1 & o & o & o & o & 1 & o & 1 & 1 & o \\
\hline \multirow[t]{3}{*}{ Mozambique } & 4.5 & 3.5 & 3 & 2.5 & 3 & 2.5 & 3 & 2 & 3 & 3 & 2.5 & 2.5 & 3 & 3 & 3 & 3 & 3 & 3 & 3.5 & 3 & 3 & 3 & 3 & 2.5 & 3 \\
\hline & - & - & - & - & - & - & - & - & - & - & - & - & - & - & 74.8 & 76.9 & 76.8 & 80.7 & 81.7 & 83.6 & 82.4 & 82.8 & 85.9 & 86.9 & 87.8 \\
\hline & o & o & o & o & o & o & o & o & o & o & o & o & 0 & o & o & o & o & o & o & o & o & o & o & 1 & o \\
\hline \multirow[t]{3}{*}{ Myanmar } & 5 & 5 & 5 & 4.5 & 4 & 5 & 4.5 & 4 & 4.5 & 3.5 & 3.5 & 4 & 4 & 4 & 4.5 & 5 & 4.5 & 4.5 & 5 & 4.5 & 4 & 4 & 4 & 4 & 5 \\
\hline & - & - & - & - & - & - & - & - & - & - & - & - & - & - & 96.5 & 97 & 100. & 101.5 & 99.4 & 98.3 & 96.2 & 94.6 & 94.3 & 94.7 & 96.3 \\
\hline & 2 & 2 & o & 2 & o & 1 & o & 1 & o & 2 & o & 1 & o & o & o & o & & o & o & o & o & 1 & 1 & o & 1 \\
\hline \multirow[t]{3}{*}{ Namibia } & 2 & 2 & 2 & 2 & 2 & 2 & 3 & 3 & 2.5 & 3 & 3 & 2.5 & 2 & 2 & 2 & 2 & 1.5 & 1.5 & 2 & 2 & 2 & 2 & 2 & 1.5 & 1.5 \\
\hline & - & - & - & - & - & - & - & - & - & - & - & - & - & - & 70.7 & 71.3 & 72.9 & 75.6 & 74.5 & 71.7 & 71 & 70.4 & 71.5 & 70.8 & 71.1 \\
\hline & o & o & o & o & o & o & 1 & 0 & o & o & o & o & o & o & o & o & o & o & o & o & o & o & o & o & o \\
\hline \multirow[t]{3}{*}{ Nepal } & 3 & 3 & 3 & 2.5 & 3.5 & 3 & 3.5 & 3 & 4 & 4 & 4.5 & 4 & 4.5 & 5 & 4.5 & 3.5 & 4 & 4.5 & 3.5 & 3.5 & 3 & 2 & 2.5 & 2.5 & 2 \\
\hline & - & - & - & - & - & - & - & - & - & - & - & - & - & - & 95.4 & 93.6 & 94.2 & 95.4 & 95.4 & 93.7 & 93 & 91.8 & 91 & 90.5 & 91.2 \\
\hline & 2 & o & o & 1 & 3 & o & o & o & o & 2 & 1 & o & 1 & o & 4 & 4 & 10 & 1 & 3 & o & o & o & o & o & o \\
\hline \multirow[t]{3}{*}{ Netherlands } & 1 & 1 & 1 & 1 & 1 & 1 & 1 & 1 & 1 & 1 & 1 & 1 & 1 & 1 & 1 & 1 & 1 & 1 & 1 & 1 & 1 & 1 & 1 & 1 & 1 \\
\hline & - & - & - & - & - & - & - & - & - & - & - & - & - & - & 28.1 & 28.6 & 27.3 & 27 & 27.9 & 28.3 & 28.1 & 26.9 & 28.6 & 26.9 & 28.2 \\
\hline & 3 & o & o & 1 & o & o & 1 & o & 1 & o & 1 & 1 & o & o & o & o & o & o & o & o & o & 1 & o & o & o \\
\hline \multirow[t]{3}{*}{ New Zealand } & 1 & 1 & 1 & 1 & 1 & 1 & 1 & 1 & 1 & 1 & 1 & 1 & 1 & 1 & 1 & 1 & 1 & 1 & 1 & 1 & 1 & 1 & 1 & 1 & 1 \\
\hline & - & - & - & - & - & - & - & - & - & - & - & - & - & - & 19.4 & 20.5 & 21.4 & $23 \cdot 3$ & 23.9 & 24.8 & 25.6 & 22.7 & 24.1 & 22.6 & 21.3 \\
\hline & o & o & o & o & o & o & o & o & o & o & o & o & o & o & o & o & o & 1 & o & o & o & o & o & o & o \\
\hline
\end{tabular}




\begin{tabular}{|c|c|c|c|c|c|c|c|c|c|c|c|c|c|c|c|c|c|c|c|c|c|c|c|c|c|}
\hline & 1992 & 1993 & 1994 & 1995 & 1996 & 1997 & 1998 & 1999 & $\begin{array}{c}200 \\
0\end{array}$ & $\begin{array}{c}200 \\
1\end{array}$ & $\begin{array}{c}200 \\
2\end{array}$ & $\begin{array}{c}200 \\
3\end{array}$ & $\begin{array}{c}200 \\
4\end{array}$ & $\begin{array}{c}200 \\
5\end{array}$ & $\begin{array}{c}200 \\
6\end{array}$ & $\begin{array}{c}200 \\
7\end{array}$ & $\begin{array}{c}200 \\
8\end{array}$ & $\begin{array}{c}200 \\
9\end{array}$ & $\begin{array}{c}201 \\
\mathbf{0}\end{array}$ & 2011 & 2012 & 2013 & 2014 & 2015 & 2016 \\
\hline \multirow[t]{3}{*}{ Nicaragua } & $3 \cdot 5$ & 3.5 & 3.5 & 3 & 3 & 3 & 3 & 2.5 & 2 & 2 & 2.5 & 3 & 2 & 2.5 & 2 & 1.5 & 2 & 2.5 & 2.5 & 2.5 & 2.5 & 3 & 2.5 & 3 & 2.5 \\
\hline & - & - & - & - & - & - & - & - & - & - & - & - & - & - & 82.4 & 80 & 81.7 & 82.6 & 82.5 & 81.2 & 79.6 & 79.2 & 78.4 & 79 & 79 \\
\hline & 1 & 1 & 1 & o & 1 & o & o & o & o & o & o & o & o & o & 0 & 0 & 0 & 0 & o & 0 & o & o & 0 & o & o \\
\hline \multirow[t]{3}{*}{ Niger } & 3 & 2.5 & 3 & 3 & 2.5 & 2 & 3 & 3 & 2 & 2 & 2 & 2.5 & 2.5 & 2 & 2 & 3.5 & 3.5 & 3 & 3 & 2.5 & 2 & 3 & 2.5 & 3.5 & 3.5 \\
\hline & - & - & - & - & - & - & - & - & - & - & - & - & - & - & 87 & 91.2 & 94.5 & 96.5 & 97.8 & 99.1 & 96.9 & 99 & 97.9 & 97.8 & 98.4 \\
\hline & o & o & 1 & 1 & o & 1 & 1 & o & o & o & o & 2 & o & o & 1 & 1 & 1 & o & o & 2 & 1 & 1 & o & 1 & 1 \\
\hline \multirow[t]{3}{*}{ Nigeria } & 3 & 4 & 4.5 & 4 & 3.5 & 3.5 & 3 & 4 & 3 & 4 & 4 & 3.5 & 4 & 4 & 4 & 3.5 & 4 & 4 & 4.5 & 4 & 4 & 4 & 4.5 & 4 & 4 \\
\hline & - & - & - & - & - & - & - & - & - & - & - & - & - & - & 94.4 & 95.6 & $95 \cdot 7$ & 99.8 & 100. & 99.9 & 101.1 & 100.7 & 99.7 & 102.5 & 103.5 \\
\hline & 1 & o & o & 1 & 1 & o & 1 & o & o & 2 & o & 3 & 2 & 3 & 1 & o & 1 & 1 & & o & 3 & 2 & o & o & 7 \\
\hline \multirow[t]{3}{*}{ Norway } & 1 & 1 & 1 & 1 & 1 & 1 & 1 & 1 & 1 & 1 & 1 & 1 & 1 & 1 & 1 & 1 & 1 & 1 & 1 & 1 & 1 & 1 & 1 & 1 & 1 \\
\hline & - & - & - & - & - & - & - & - & - & - & - & - & - & - & 16.8 & 17.1 & 16.8 & 18.3 & 18.7 & 20.4 & 23.9 & 21.5 & 23 & 20.9 & 21.2 \\
\hline & o & o & o & o & o & o & 1 & o & o & 1 & o & 1 & 1 & 0 & o & o & o & 2 & 1 & o & o & o & o & o & o \\
\hline \multirow[t]{3}{*}{ Oman } & - & 1 & 2 & 2 & 2 & 2 & 2 & 2 & 1 & 1 & 2 & 1 & 2 & 1 & 1.5 & 1 & 1 & 1 & 1 & 1.5 & 2 & 3 & 2 & 1.5 & 2.5 \\
\hline & - & - & - & - & - & - & - & - & - & - & - & - & - & - & 43.8 & 45.5 & 47.4 & 47.2 & 48.7 & 49.3 & 51.7 & 52 & 53.1 & 52 & 51.6 \\
\hline & o & o & o & o & o & o & o & o & o & o & o & o & o & o & o & o & o & o & o & o & o & o & o & o & o \\
\hline \multirow[t]{3}{*}{ Pakistan } & 4 & 3.5 & 4 & 4.5 & 4 & 3.5 & 4 & 4.5 & 3.5 & 4 & 4 & 4 & 3.5 & 4 & 4 & 4 & 4 & 4.5 & 5 & 5 & 5 & 5 & 4.5 & 4 & 3.5 \\
\hline & - & - & - & - & - & - & - & - & - & - & - & - & - & - & 103.1 & 100.1 & 103.8 & 104.1 & 102.5 & 102.3 & 101.6 & 102.9 & 103 & 103 & 101.7 \\
\hline & 4 & 2 & 2 & 2 & 5 & 4 & 2 & 2 & 5 & 7 & 11 & 5 & 5 & 1 & 1 & 13 & 5 & 6 & 2 & 4 & 8 & 5 & 10 & 1 & o \\
\hline \multirow{3}{*}{$\begin{array}{l}\text { Palestine, State } \\
\text { of }\end{array}$} & - & - & 4 & 4 & 3.5 & 3.5 & 3.5 & 3.5 & 4 & 4 & 4 & 3.5 & 3.5 & 4 & 4 & 3.5 & 4 & 4 & 4 & 4 & 4 & 3 & 3 & 4 & 3 \\
\hline & - & - & - & - & - & - & - & - & - & - & - & - & - & - & - & - & - & - & - & - & - & - & - & - & - \\
\hline & 3 & 1 & 1 & o & o & o & o & 3 & 5 & 3 & o & 1 & o & 3 & 7 & 1 & 3 & 1 & 1 & 1 & 4 & 3 & 3 & o & 1 \\
\hline \multirow[t]{3}{*}{ Panama } & 2 & 2 & 1.5 & 2 & 2.5 & 2 & 2 & 2 & 2 & 2 & 1 & 2 & 2 & 1 & 1 & 1 & 2 & 1 & 2 & 1.5 & 2 & 2 & 1 & 1 & 1 \\
\hline & - & - & - & - & - & - & - & - & - & - & - & - & - & - & 59.6 & 59.4 & 58.6 & 59.7 & 59.3 & 57.8 & 56.1 & 55.8 & $55 \cdot 7$ & 54.6 & 53.2 \\
\hline & 1 & o & 1 & o & o & o & o & o & 0 & o & o & o & o & o & o & o & o & o & o & o & o & o & o & o & o \\
\hline \multirow{3}{*}{$\begin{array}{l}\text { Papua New } \\
\text { Guinea }\end{array}$} & 4 & 3.5 & 3.5 & 3 & 3 & 2.5 & 2 & 2.5 & 2 & 2 & 2.5 & 3 & 2.5 & 3 & 3 & 2.5 & 2.5 & 3 & 3 & 3 & 2 & 2.5 & 3 & 2 & 2 \\
\hline & - & - & - & - & - & - & - & - & - & - & - & - & - & - & 84.6 & 85.1 & 84.6 & 84.1 & 83.9 & 84.2 & 83.7 & 84.9 & 84.1 & 83.4 & 86.2 \\
\hline & o & o & o & o & o & o & o & o & o & o & o & o & o & o & 1 & o & o & o & o & o & o & o & o & o & o \\
\hline
\end{tabular}




\begin{tabular}{|c|c|c|c|c|c|c|c|c|c|c|c|c|c|c|c|c|c|c|c|c|c|c|c|c|c|}
\hline & 1992 & 1993 & 1994 & 1995 & 1996 & 1997 & 1998 & 1999 & $\begin{array}{c}200 \\
\mathbf{0}\end{array}$ & $\begin{array}{c}200 \\
1\end{array}$ & $\begin{array}{c}200 \\
2\end{array}$ & $\begin{array}{c}200 \\
3\end{array}$ & $\begin{array}{c}200 \\
4\end{array}$ & $\begin{array}{c}200 \\
5\end{array}$ & $\begin{array}{c}200 \\
6\end{array}$ & $\begin{array}{c}200 \\
7\end{array}$ & $\begin{array}{c}200 \\
8\end{array}$ & $\begin{array}{c}200 \\
9\end{array}$ & $\begin{array}{c}201 \\
\mathbf{0}\end{array}$ & 2011 & 2012 & 2013 & 2014 & 2015 & 2016 \\
\hline \multirow[t]{3}{*}{ Paraguay } & 2.5 & 2.5 & 2.5 & 2.5 & 3 & 2 & 3 & 3 & 3 & 2 & 2.5 & 2.5 & 2 & 2.5 & 3 & 3 & 3 & 2 & $\overline{3}$ & 2.5 & 2 & 3 & 2 & 1.5 & 1.5 \\
\hline & - & - & - & - & - & - & - & - & - & - & - & - & - & - & 72 & 72.4 & 72.3 & 72 & 72.1 & 72.4 & 70.9 & 71.8 & 71.6 & 71.3 & 72.6 \\
\hline & o & o & o & o & o & o & o & o & o & o & o & o & o & o & o & o & 1 & o & o & o & o & o & o & o & o \\
\hline \multirow[t]{3}{*}{ Peru } & 5 & 4 & 4.5 & 4 & 4 & 3.5 & 3.5 & 3.5 & 3 & 2 & 2.5 & 3 & 2.5 & 2.5 & 3 & 1.5 & 2 & 2.5 & 2.5 & 2.5 & 3 & 3 & 3 & 2 & 2 \\
\hline & - & - & - & - & - & - & - & - & - & - & - & - & - & - & 79.2 & 76.4 & 77.5 & 77.1 & 76.9 & 73.6 & 73.5 & 72.3 & 72.9 & 72 & 72 \\
\hline & 1 & o & o & o & o & o & o & o & 1 & o & o & 1 & o & o & o & o & o & o & o & o & o & o & o & o & o \\
\hline \multirow[t]{3}{*}{ Philippines } & 4 & 4 & 3.5 & 3.5 & 3 & 3 & 3 & 3.5 & 4 & 3.5 & 3 & 4 & 4 & 4 & 4 & 4 & 4 & 4 & 4 & 4 & 4 & 4 & 3.5 & 4 & 5 \\
\hline & - & - & - & - & - & - & - & - & - & - & - & - & - & - & 79.2 & 83.2 & 83.4 & 85.8 & 87.1 & 85 & 83.2 & 82.8 & 85.3 & 86.2 & 84.7 \\
\hline & o & 1 & o & 2 & o & 2 & 1 & 1 & o & 4 & 1 & o & 1 & o & 4 & o & 1 & o & 1 & o & 1 & 3 & 3 & 1 & o \\
\hline \multirow[t]{3}{*}{ Poland } & 1.5 & 1 & 1 & 1 & 1 & 1 & 2 & 2 & 1.5 & 1.5 & 2 & 1 & 2 & 1.5 & 1.5 & 2 & 1.5 & 1.5 & 1.5 & 1.5 & 1.5 & 1 & 1 & 1 & 1.5 \\
\hline & - & - & - & - & - & - & - & - & - & - & - & - & - & - & 47.9 & 47.6 & 47.6 & 49.6 & 49 & 46.8 & 44.3 & 40.9 & 42.1 & 39.8 & 40.7 \\
\hline & o & o & o & o & o & o & o & o & o & o & o & o & o & o & o & o & o & o & o & o & o & o & o & o & o \\
\hline \multirow[t]{3}{*}{ Portugal } & 1 & 1 & 1 & 1 & 1 & 1 & 1 & 1 & 1 & 1 & 2 & 2 & 2 & 2 & 2 & 2 & 2 & 2 & 1.5 & 2 & 1.5 & 2 & 1.5 & 1 & 1.5 \\
\hline & - & - & - & - & - & - & - & - & - & - & - & - & - & - & 32.7 & 32.4 & 31.8 & 32.7 & 33.1 & 32.3 & 34.2 & 32.6 & 33.1 & 29.7 & 29.2 \\
\hline & o & o & o & o & o & o & o & o & o & o & o & o & o & o & o & o & o & o & o & o & o & o & o & o & o \\
\hline \multirow[t]{3}{*}{ Qatar } & 1 & 1 & 1.5 & 1 & 1.5 & 1.5 & 1.5 & 1.5 & 1.5 & 1.5 & 1.5 & 1 & 1 & 1.5 & 1.5 & 2 & 1.5 & 1.5 & 1 & 1 & 1 & 2 & 1.5 & 1.5 & 2 \\
\hline & - & - & - & - & - & - & - & - & - & - & - & - & - & - & - & 53.6 & 52.7 & 51.9 & 51.8 & 49.5 & 48 & 47.1 & 48.9 & 46.3 & 45.1 \\
\hline & o & o & o & o & o & o & o & 1 & o & o & o & o & o & o & o & o & o & o & o & o & o & o & o & o & o \\
\hline \multirow[t]{3}{*}{ Romania } & 2 & 2 & 2.5 & 2 & 2 & 2.5 & 2.5 & 3 & 2.5 & 2.5 & 2.5 & 2 & 2.5 & 2.5 & 2.5 & 2.5 & 3 & 2.5 & 2 & 2 & 2 & 1.5 & 2 & 2 & 2 \\
\hline & - & - & - & - & - & - & - & - & - & - & - & - & - & - & 62.6 & 60.9 & 59.9 & 61.3 & 60.2 & 59.8 & 59.5 & 57.4 & 56.9 & 54.2 & 52.9 \\
\hline & o & o & o & 1 & o & o & o & o & o & o & o & o & o & o & o & o & o & o & o & o & o & o & o & o & o \\
\hline \multirow[t]{3}{*}{ Russia } & 2.5 & 2.5 & 4.5 & 4.5 & 4 & 3.5 & 4.5 & 4 & 4 & 4 & 4 & 4 & 4 & 4 & 4 & 4 & 4 & 4 & 4 & 4 & 4 & 3.5 & 4 & 3.5 & 4 \\
\hline & - & - & - & - & - & - & - & - & - & - & - & - & - & - & 87.1 & 81.2 & 79.7 & 80.8 & 79 & 77.7 & 77.1 & 77.1 & 76.5 & 80 & 81 \\
\hline & 1 & 2 & 1 & 1 & 3 & 1 & 5 & 4 & 1 & 3 & 7 & 3 & 1 & 2 & 3 & 1 & 3 & 4 & o & 2 & 3 & 6 & 3 & 1 & o \\
\hline \multirow[t]{3}{*}{ Rwanda } & 4 & 4 & 5 & 5 & 5 & 5 & 5 & 4.5 & 4 & 4.5 & 4 & 3.5 & 3.5 & 2.5 & 2.5 & 3 & 2 & 2.5 & 2.5 & 3 & 2.5 & 2.5 & 3.5 & 2.5 & 3.5 \\
\hline & - & - & - & - & - & - & - & - & - & - & - & - & - & - & 92.9 & 89.2 & 88 & 89 & 88.7 & 91 & 89.3 & 89.3 & 90.5 & 90.2 & 91.3 \\
\hline & 1 & o & o & o & o & o & 1 & o & o & o & o & o & o & o & 1 & o & o & o & o & o & 1 & o & o & o & o \\
\hline
\end{tabular}




\begin{tabular}{|c|c|c|c|c|c|c|c|c|c|c|c|c|c|c|c|c|c|c|c|c|c|c|c|c|c|}
\hline & 1992 & 1993 & 1994 & 1995 & 1996 & 1997 & 1998 & 1999 & $\begin{array}{c}200 \\
\text { o }\end{array}$ & $\begin{array}{c}200 \\
1\end{array}$ & $\begin{array}{c}200 \\
2\end{array}$ & $\begin{array}{c}200 \\
3\end{array}$ & $\begin{array}{c}200 \\
4\end{array}$ & $\begin{array}{c}200 \\
5\end{array}$ & $\begin{array}{c}200 \\
6\end{array}$ & $\begin{array}{c}200 \\
7\end{array}$ & $\stackrel{200}{8}$ & $\begin{array}{c}200 \\
9\end{array}$ & $\begin{array}{c}201 \\
\mathbf{0}\end{array}$ & 2011 & 2012 & 2013 & 2014 & 2015 & 2016 \\
\hline \multirow[t]{3}{*}{ Samoa } & 1 & $\overline{1}$ & $\overline{1}$ & $\overline{1}$ & $\overline{1}$ & 1 & $\overline{1}$ & 1 & 1 & $\overline{1}$ & 1 & 1 & $\overline{1}$ & $\overline{1}$ & $\overline{1}$ & $\overline{1}$ & 1 & $\overline{1}$ & 2 & 1 & $\overline{1}$ & $\overline{1}$ & 1 & 1 & 1 \\
\hline & - & - & - & - & - & - & - & - & - & - & - & - & - & - & - & 73.8 & 72.4 & 71.4 & 71.1 & 69.5 & 68.5 & 68.7 & 69.3 & 68.2 & 67.6 \\
\hline & o & o & o & o & o & o & o & o & o & o & o & o & o & o & o & o & o & o & o & o & o & o & o & o & o \\
\hline \multirow{3}{*}{$\begin{array}{l}\text { Sao Tome and } \\
\text { Principe }\end{array}$} & 1 & 1 & 1 & 1 & 1 & 1 & 1 & 1 & 1 & 1 & 2 & 2 & 1 & 1 & 1 & 1 & 1 & 1 & 1 & 1 & 1 & 1 & 1 & 1 & 1 \\
\hline & - & - & - & - & - & - & - & - & - & - & - & - & - & - & - & 78.6 & 78.3 & 76.7 & 75.8 & 74.5 & 73.9 & 74.6 & 75.8 & 73.7 & 72.9 \\
\hline & o & o & o & o & o & o & o & o & o & o & o & o & o & o & o & o & o & o & o & o & o & o & o & o & o \\
\hline \multirow[t]{3}{*}{ Saudi Arabia } & 2.5 & 3 & 2.5 & 3.5 & 2.5 & 3 & 3.5 & 3.5 & 3 & 3 & 3 & 3 & 3 & 3 & 3 & 3.5 & 3.5 & 4 & 3.5 & 3.5 & 3 & 3 & 3 & 3.5 & 3.5 \\
\hline & - & - & - & - & - & - & - & - & - & - & - & - & - & - & 77.2 & 76.5 & 76.9 & 77.5 & 77.5 & 75.2 & 73.4 & 72.7 & 73.1 & 71.6 & 72.2 \\
\hline & o & o & o & 1 & o & o & o & 1 & 1 & o & o & 3 & 1 & o & 1 & o & o & o & o & o & 1 & 2 & o & 2 & o \\
\hline \multirow[t]{3}{*}{ Senegal } & 3 & 3 & 2.5 & 3.5 & 2.5 & 3 & 3.5 & 3 & 2.5 & 2.5 & 2.5 & 2.5 & 1.5 & 2 & 3 & 3 & 2.5 & 3 & 2.5 & 2.5 & 3 & 2 & 2.5 & 2 & 2 \\
\hline & - & - & - & - & - & - & - & - & - & - & - & - & - & - & 66.1 & 66.9 & 70.9 & 74.2 & 74.6 & 76.8 & 79.3 & 81.4 & 82.8 & 82.9 & 83.6 \\
\hline & o & o & o & o & o & o & o & o & o & 1 & o & o & o & o & o & o & o & o & o & o & o & o & o & 1 & o \\
\hline \multirow[t]{3}{*}{ Serbia } & - & - & - & - & - & - & - & - & - & - & - & - & - & - & - & 2 & 2 & 2 & 2 & 2 & 2 & 1.5 & 1.5 & 1.5 & 1.5 \\
\hline & - & - & - & - & - & - & - & - & - & - & - & - & - & - & 83.8 & 81.1 & 80.1 & 77.8 & 74.4 & 75 & 74.4 & 72 & 73.8 & 72 & 79.2 \\
\hline & o & o & o & o & o & o & 1 & 0 & o & 1 & o & o & o & o & 1 & o & 1 & o & o & o & o & o & o & o & o \\
\hline \multirow[t]{3}{*}{$\begin{array}{l}\text { Seychelles } \\
\end{array}$} & 2 & 1 & 1 & 1 & 1 & 2 & 1 & 1 & 1 & 1 & 2 & 2 & 2 & 1 & 1 & 1 & 2 & 2 & 1 & 1 & 1 & 2 & 2 & 2 & 2 \\
\hline & - & - & - & - & - & - & - & - & - & - & - & - & - & - & - & 71.3 & 69.5 & 67.7 & 67.9 & 67 & 65.1 & 64 & 63.7 & 62.1 & 60.2 \\
\hline & o & o & o & o & o & o & o & o & o & o & o & o & o & o & o & o & o & o & o & o & o & o & o & o & o \\
\hline \multirow[t]{3}{*}{ Sierra Leone } & 4 & 4 & 5 & 4 & 5 & 4.5 & 5 & 5 & 4.5 & 4 & 2.5 & 3.5 & 2.5 & 2.5 & 2.5 & 2.5 & 2.5 & 2 & 2.5 & 2 & 2.5 & 3 & 2 & 2 & 2 \\
\hline & - & - & - & - & - & - & - & - & - & - & - & - & - & - & 96.6 & 93.4 & 92.3 & 92.1 & 93.6 & 92.1 & 90.4 & 91.2 & 89.9 & 91.9 & 91 \\
\hline & o & o & o & o & o & o & o & 2 & o & o & o & o & o & o & o & o & o & o & o & o & o & o & o & o & o \\
\hline \multirow[t]{3}{*}{ Singapore } & 1 & 1.5 & 1.5 & 1.5 & 1.5 & 1.5 & 2 & 2 & 1.5 & 2 & 2 & 2 & 2 & 2 & 1.5 & 1.5 & 1 & 1.5 & 1.5 & 1.5 & 1.5 & 1 & 1.5 & 1.5 & 1.5 \\
\hline & - & - & - & - & - & - & - & - & - & - & - & - & - & - & 30.8 & 33 & 33.3 & 33.8 & 34.8 & 35.1 & 35.6 & 34 & 35.9 & 34.4 & 32.9 \\
\hline & o & o & o & o & o & o & o & o & o & o & o & o & o & o & o & o & 1 & o & o & o & o & o & o & o & o \\
\hline \multirow[t]{3}{*}{ Slovak Republic } & 1 & 1 & 1 & 1 & 1 & 1 & 1 & 2 & 1.5 & 1.5 & 2 & 1 & 2 & 1 & 2 & 2 & 1.5 & 1.5 & 1.5 & 1.5 & 1 & 1 & 1 & 2 & 1.5 \\
\hline & - & - & - & - & - & - & - & - & - & - & - & - & - & - & 49.9 & $49 \cdot 3$ & 48.8 & 48.6 & 48.8 & 47.1 & 47.4 & $45 \cdot 3$ & $45 \cdot 3$ & 42.6 & 44.9 \\
\hline & o & o & o & o & o & o & o & o & o & o & o & o & o & o & o & o & o & o & o & o & o & o & o & o & o \\
\hline
\end{tabular}




\begin{tabular}{|c|c|c|c|c|c|c|c|c|c|c|c|c|c|c|c|c|c|c|c|c|c|c|c|c|c|}
\hline & 1992 & 1993 & 1994 & 1995 & 1996 & 1997 & 1998 & 1999 & $\begin{array}{c}200 \\
\mathbf{0}\end{array}$ & $\begin{array}{c}200 \\
1\end{array}$ & $\begin{array}{c}200 \\
2\end{array}$ & $\begin{array}{c}200 \\
3\end{array}$ & $\begin{array}{c}200 \\
4\end{array}$ & $\begin{array}{c}200 \\
5\end{array}$ & $\underset{6}{200}$ & $\begin{array}{c}200 \\
7\end{array}$ & $\stackrel{200}{8}$ & $\begin{array}{c}200 \\
9\end{array}$ & $\begin{array}{c}201 \\
0\end{array}$ & 2011 & 2012 & 2013 & 2014 & 2015 & 2016 \\
\hline \multirow[t]{3}{*}{ Slovenia } & - & - & - & - & - & 1 & 1 & 1 & 1.5 & $\overline{1}$ & 1 & 1.5 & $\overline{1}$ & $\overline{1}$ & $\overline{1}$ & 1.5 & $\overline{1}$ & $\overline{1}$ & $\overline{1}$ & $\overline{1}$ & $\overline{1}$ & $\overline{1}$ & 1 & 1 & $\overline{1}$ \\
\hline & - & - & - & - & - & - & - & - & - & - & - & - & - & - & 36.8 & 37.5 & 37.1 & 36.3 & 36 & 35.5 & 34 & 32.3 & 32.6 & 31.5 & 33.9 \\
\hline & o & o & o & o & o & o & o & o & o & o & o & o & o & o & o & o & o & o & o & o & o & o & o & o & o \\
\hline \multirow[t]{3}{*}{ Solomon Islands } & 1 & 1 & 1 & 1 & 1 & 1 & 1 & 2.5 & 3.5 & 3 & 3 & 3 & 2 & 1.5 & 1 & 1 & 1 & 1 & 1 & 1 & 1 & 1 & 1 & 1 & 1 \\
\hline & - & - & - & - & - & - & - & - & - & - & - & - & - & - & - & 92 & 92.4 & 89.6 & 88.6 & 85.9 & 85.6 & 85.2 & 86.4 & 85.9 & 85.3 \\
\hline & o & o & o & o & 1 & o & o & 2 & o & o & 1 & o & o & o & o & o & o & o & o & o & o & o & o & o & o \\
\hline \multirow[t]{3}{*}{ Somalia } & 5 & 4.5 & 5 & 4.5 & 4.5 & 3.5 & 3.5 & 4 & 4 & 4 & 4 & 4 & 4 & 4 & 4 & 5 & 5 & 4.5 & 4.5 & 5 & 4.5 & 4.5 & 5 & 4.5 & 4.5 \\
\hline & - & - & - & - & - & - & - & - & - & - & - & - & - & - & 105.9 & 111.1 & 114.2 & 114.7 & 114.3 & 113.4 & 114.9 & 113.9 & 112.6 & 114 & 114 \\
\hline & 3 & o & o & o & o & o & o & o & 1 & 1 & 1 & o & 2 & o & 2 & 1 & 3 & 2 & o & 2 & 1 & 1 & o & 1 & 1 \\
\hline \multirow[t]{3}{*}{ South Africa } & 4.5 & 5 & 4.5 & 4 & 4 & 3.5 & 4 & 3 & 3 & 3.5 & 3 & 3 & 3 & 3 & 3 & 3.5 & 3 & 3 & 3 & 3 & 3.5 & 3 & 3 & 3.5 & 3.5 \\
\hline & - & - & - & - & - & - & - & - & - & - & - & - & - & - & 55.7 & 57.4 & 62.7 & 67.4 & 67.9 & 67.6 & 66.8 & 67.6 & 66.6 & 67 & 69.9 \\
\hline & 2 & o & 1 & 1 & 1 & o & 1 & 1 & o & o & 1 & o & o & o & o & o & o & 1 & o & o & o & o & o & o & o \\
\hline \multirow[t]{3}{*}{ South Sudan } & - & - & - & - & - & - & - & - & - & - & - & - & - & - & - & - & - & - & - & - & 4.5 & 4 & 4.5 & 5 & 5 \\
\hline & - & - & - & - & - & - & - & - & - & - & - & - & - & - & - & - & - & - & - & - & 108. & 110.6 & 112.9 & 114.5 & 113.8 \\
\hline & o & o & o & o & o & o & o & 1 & o & o & o & o & o & o & o & o & o & 1 & 1 & o & 4 & o & 1 & o & o \\
\hline \multirow[t]{3}{*}{ Spain } & 1.5 & 1.5 & 2 & 2 & 2 & 2 & 2 & 1.5 & 1.5 & 2 & 3 & 2.5 & 2.5 & 2 & 2 & 2.5 & 2.5 & 2 & 1.5 & 1.5 & 1.5 & 1 & 1.5 & 1.5 & 2 \\
\hline & - & - & - & - & - & - & - & - & - & - & - & - & - & - & 37.4 & 39.2 & 41.6 & $43 \cdot 3$ & 43.5 & 43.1 & 42.8 & 44.4 & 43.1 & 40.9 & 39.8 \\
\hline & o & 1 & o & o & o & 0 & 1 & o & 1 & 2 & 1 & 2 & 1 & 2 & 1 & 1 & o & o & o & o & o & 1 & 1 & o & o \\
\hline \multirow[t]{3}{*}{ Sri Lanka } & 4.5 & 4.5 & 3.5 & 4.5 & 4.5 & 4 & 4 & 4 & 4.5 & 4 & 3 & 3 & 3.5 & 4.5 & 5 & 5 & 4.5 & 5 & 4.5 & 4.5 & 4 & 3.5 & 3.5 & 3 & 3 \\
\hline & - & - & - & - & - & - & - & - & - & - & - & - & - & - & 92.4 & 93.1 & 95.6 & 96.7 & 95.7 & 93.1 & 92.2 & 92.9 & 92.6 & 90.7 & 87.7 \\
\hline & o & o & 1 & o & o & 1 & o & o & 1 & o & o & o & 2 & o & o & o & o & 1 & o & o & 1 & 1 & o & o & o \\
\hline \multirow[t]{3}{*}{ Sudan } & 5 & 5 & 5 & 4.5 & 4.5 & 4.5 & 4.5 & 5 & 5 & 5 & 4.5 & 5 & 5 & 5 & 5 & 5 & 5 & 5 & 5 & 5 & 5 & 5 & 5 & 5 & 5 \\
\hline & - & - & - & - & - & - & - & - & - & - & - & - & - & - & 112.3 & 113.7 & 113 & 112.4 & 111.8 & 108.7 & 109.4 & 111 & 110.1 & 110.8 & 111.5 \\
\hline & o & o & 1 & 1 & o & o & 1 & 3 & 1 & o & o & 4 & o & o & 1 & o & 1 & 1 & 1 & 1 & 1 & 1 & o & o & o \\
\hline \multirow[t]{3}{*}{ Suriname } & 1.5 & 2 & 2 & 2 & 1.5 & 2 & 1 & 1 & 2 & 2 & 2 & 1.5 & 2 & 2 & 2 & 2 & 1.5 & 1.5 & 2 & 2 & 1.5 & 2 & 1.5 & 1 & 2 \\
\hline & - & - & - & - & - & - & - & - & - & - & - & - & - & - & - & 73.9 & 72.4 & 73.2 & 72.5 & 71.1 & 71.2 & 71.2 & 70.6 & 68.4 & 66.7 \\
\hline & o & o & 1 & o & o & o & o & o & o & o & o & o & o & o & o & o & o & o & o & o & o & o & o & o & o \\
\hline
\end{tabular}




\begin{tabular}{|c|c|c|c|c|c|c|c|c|c|c|c|c|c|c|c|c|c|c|c|c|c|c|c|c|c|}
\hline & 1992 & 1993 & 1994 & 1995 & 1996 & 1997 & 1998 & 1999 & $\begin{array}{c}200 \\
\mathbf{0}\end{array}$ & $\begin{array}{c}200 \\
1\end{array}$ & $\begin{array}{c}200 \\
2\end{array}$ & $\begin{array}{c}200 \\
3\end{array}$ & $\begin{array}{c}200 \\
4\end{array}$ & $\begin{array}{c}200 \\
5\end{array}$ & $\begin{array}{c}200 \\
6\end{array}$ & $\begin{array}{c}200 \\
7\end{array}$ & $\begin{array}{c}200 \\
8\end{array}$ & $\begin{array}{c}200 \\
9\end{array}$ & $\begin{array}{c}201 \\
\mathbf{0}\end{array}$ & 2011 & 2012 & 2013 & 2014 & 2015 & 2016 \\
\hline \multirow[t]{3}{*}{ Swaziland } & 1.5 & 2.5 & 2.5 & 2 & 1.5 & 2 & 2 & 2 & 2 & 2 & 2 & 2 & 2 & 2.5 & 3 & 3 & 3 & 3 & 3 & 2.5 & 3 & 3 & 2.5 & 2.5 & 2.5 \\
\hline & - & - & - & - & - & - & - & - & - & - & - & - & - & - & - & 81.3 & 80 & 82.4 & 82.8 & 82.5 & 83.5 & 85.6 & 85.8 & 86.2 & 87.6 \\
\hline & o & o & o & o & o & o & 1 & 0 & o & o & o & 1 & 0 & o & 1 & 0 & 1 & 0 & o & 0 & 0 & o & 0 & o & 0 \\
\hline \multirow[t]{3}{*}{ Sweden } & 1 & 1 & 1 & 1 & 1 & 1 & 1 & 1 & 1 & 1 & 1.5 & 1 & 1 & 1 & 1 & 1 & 1 & 1 & 1 & 1 & 1 & 1 & 1 & 1 & 1 \\
\hline & - & - & - & - & - & - & - & - & - & - & - & - & - & - & 18.2 & 19.3 & 19.8 & 20.6 & 20.9 & 22.8 & 21.3 & 19.7 & 21.4 & 20.2 & 22.6 \\
\hline & o & 1 & 1 & o & o & 2 & 2 & 1 & o & o & 1 & o & 1 & 2 & o & o & 1 & 2 & o & o & o & o & o & o & o \\
\hline \multirow[t]{3}{*}{ Switzerland } & 1.5 & 1.5 & 1.5 & 1 & 1 & 1 & 1 & 1 & 1 & 1 & 2 & 2 & 2 & 1.5 & 1.5 & 2 & 2 & 1.5 & 1 & 1 & 1 & 1 & 1 & 1 & 1.5 \\
\hline & - & - & - & - & - & - & - & - & - & - & - & - & - & - & 18.7 & 20.2 & 20.3 & 21.2 & 21.8 & 23.2 & $23 \cdot 3$ & 21.5 & $23 \cdot 3$ & 22.3 & 21.8 \\
\hline & o & o & o & 1 & o & o & o & 1 & o & 1 & o & 1 & o & o & 1 & o & o & 2 & o & o & 1 & o & o & o & o \\
\hline \multirow[t]{3}{*}{ Syria } & 3 & 3.4 & 4 & 3 & 3 & 3 & 3 & 3 & 3 & 3 & 3 & 3 & 3 & 3 & 3 & 3.5 & 4 & 4 & 4 & 5 & 5 & 5 & 5 & 5 & 5 \\
\hline & - & - & - & - & - & - & - & - & - & - & - & - & - & - & 88.6 & 88.6 & 90.1 & 89.8 & 87.9 & 85.9 & 94.5 & 97.4 & 101.6 & 107.8 & 110.8 \\
\hline & o & o & 1 & 1 & o & o & 1 & 2 & o & 2 & o & 5 & 3 & 3 & 2 & 1 & 2 & 3 & o & 8 & 27 & 31 & 15 & 6 & 4 \\
\hline \multirow[t]{3}{*}{ Taiwan } & 1.5 & 2 & 2 & 1.5 & 1 & 2 & 1.5 & 1 & 1 & 2 & 1 & 1.5 & 1 & 1 & 1 & 1 & 1 & 1 & 1 & 1 & 1 & 1 & 1.5 & 1 & 1 \\
\hline & - & - & - & - & - & - & - & - & - & - & - & - & - & - & - & - & - & - & - & - & - & - & - & - & - \\
\hline & o & o & o & o & o & o & o & o & o & o & o & o & o & o & o & o & o & o & o & o & o & o & o & o & o \\
\hline \multirow[t]{3}{*}{ Tajikistan } & 4.5 & 4.5 & 3 & 2.5 & 3 & 2.5 & 3 & 3 & 2.5 & 2.5 & 2.5 & 2.5 & 2.5 & 3 & 3 & 3 & 2.5 & 2 & 2 & 2.5 & 3 & 3 & 3 & 3 & 3.5 \\
\hline & - & - & - & - & - & - & - & - & - & - & - & - & - & - & 87.7 & 88.7 & 88.9 & 90.3 & 89.2 & 88.3 & 85.7 & 85.2 & 84.6 & 83.4 & 83.8 \\
\hline & o & 1 & 1 & 2 & o & 1 & o & o & 1 & o & o & 1 & o & o & o & o & o & o & 1 & o & o & 2 & o & o & o \\
\hline \multirow[t]{3}{*}{ Tanzania } & 1.5 & 2 & 2 & 2.5 & 3 & 3 & 3 & 2.5 & 3 & 3 & 2.5 & 3 & 2.5 & 3 & 2.5 & 2.5 & 2.5 & 2 & 2.5 & 2.5 & 2.5 & 3 & 3 & 2.5 & 3 \\
\hline & - & - & - & - & - & - & - & - & - & - & - & - & - & - & 78.3 & 79.3 & 79.1 & 81.1 & 81.2 & 81.3 & 80.4 & 81.1 & 80.8 & 80.8 & 81.8 \\
\hline & o & 1 & o & 1 & o & o & o & o & o & 1 & 1 & o & o & o & 1 & o & o & o & o & o & o & o & o & o & 1 \\
\hline \multirow[t]{3}{*}{ Thailand } & 3.5 & 2.5 & 3 & 2 & 2.5 & 2.5 & 2.5 & 2 & 2.5 & 2.5 & 2.5 & 3 & 3.5 & 4 & 4 & 4 & 3.5 & 3 & 4 & 3.5 & 3.5 & 3 & 3 & 3.5 & 3.5 \\
\hline & - & - & - & - & - & - & - & - & - & - & - & - & - & - & 74.9 & 76 & 75.6 & 79.2 & 78.8 & 78.3 & 77 & 75.1 & 77 & 79.1 & 78.8 \\
\hline & 1 & o & 1 & 1 & o & 1 & o & 1 & 1 & 2 & 1 & o & o & o & 2 & o & o & o & o & o & 1 & o & o & o & o \\
\hline \multirow[t]{3}{*}{ Timor-Leste } & - & - & - & - & - & - & - & - & 2 & 2 & 2 & 2 & 2.5 & 2 & 3.5 & 3 & 2 & 2 & 2 & 2 & 2 & 2 & 2 & 2.5 & 2 \\
\hline & - & - & - & - & - & - & - & - & - & - & - & - & - & - & - & 94.9 & 93.8 & 97.2 & 98.2 & 94.9 & 92.7 & 91.5 & 91 & 90.5 & 90.8 \\
\hline & o & o & o & o & o & o & o & o & o & o & o & o & o & o & o & o & o & o & o & o & o & o & o & o & o \\
\hline
\end{tabular}




\begin{tabular}{|c|c|c|c|c|c|c|c|c|c|c|c|c|c|c|c|c|c|c|c|c|c|c|c|c|c|}
\hline & 1992 & 1993 & 1994 & 1995 & 1996 & 1997 & 1998 & 1999 & $\begin{array}{c}200 \\
\mathbf{0}\end{array}$ & $\begin{array}{c}200 \\
1\end{array}$ & $\begin{array}{c}200 \\
2\end{array}$ & $\begin{array}{c}200 \\
3\end{array}$ & $\begin{array}{c}200 \\
4\end{array}$ & $\begin{array}{c}200 \\
5\end{array}$ & $\underset{6}{200}$ & $\begin{array}{c}200 \\
7\end{array}$ & $\stackrel{200}{8}$ & $\begin{array}{c}200 \\
9\end{array}$ & $\begin{array}{c}201 \\
\mathbf{0}\end{array}$ & 2011 & 2012 & 2013 & 2014 & 2015 & 2016 \\
\hline \multirow[t]{3}{*}{ Togo } & 2.5 & 4.5 & 3.5 & 2.5 & 2.5 & 2.5 & 4 & 3 & $\begin{array}{l}2.5 \\
\end{array}$ & 2.5 & 2 & 3 & 3 & 4.5 & 3 & 3 & $\begin{array}{l}2.5 \\
\end{array}$ & $\begin{array}{l}2.5 \\
\end{array}$ & $\begin{array}{l}2.5 \\
\end{array}$ & 3 & 2.5 & 1 & $\begin{array}{l}1.5 \\
\end{array}$ & 2 & 2 \\
\hline & - & - & - & - & - & - & - & - & - & - & - & - & - & - & 88.3 & 86.6 & 86.8 & 87.2 & 88.1 & 89.4 & 87.5 & 87.8 & 87.8 & 86.8 & 85.8 \\
\hline & o & o & o & o & o & o & o & o & o & o & o & o & o & o & o & o & o & o & o & o & o & o & o & o & o \\
\hline \multirow{3}{*}{$\begin{array}{l}\text { Trinidad and } \\
\text { Tobago }\end{array}$} & 1.5 & 2 & $\begin{array}{ll}1.5 \\
\end{array}$ & 1.5 & 1.5 & 1.5 & $\begin{array}{ll}1.5 \\
\end{array}$ & 1.5 & 1.5 & 1.5 & 2 & 2 & $\begin{array}{l}2.5 \\
\end{array}$ & 3 & $\begin{array}{l}2.5 \\
\end{array}$ & 2 & 2.5 & $\begin{array}{l}2.5 \\
\end{array}$ & $\begin{array}{l}2.5 \\
\end{array}$ & 2.5 & 2 & 2 & 2 & 2 & 2 \\
\hline & - & - & - & - & - & - & - & - & - & - & - & - & - & - & - & 67.6 & 67 & 66.7 & 66.1 & 63.7 & 64.4 & 62.6 & 61.3 & 58.7 & 57.8 \\
\hline & o & o & o & o & o & o & o & o & o & o & o & o & 0 & o & o & 0 & o & o & o & o & o & o & o & o & o \\
\hline \multirow[t]{3}{*}{ Tunisia } & 3 & 3 & 3.5 & 3 & 2.5 & 3.5 & 3 & 3 & 3 & 3 & 2.5 & 3 & 3 & $\begin{array}{l}2.5 \\
\end{array}$ & 3 & 3 & 3 & 3 & 3 & 2 & 2.5 & 3 & 2.5 & $\begin{array}{l}2.5 \\
\end{array}$ & 3 \\
\hline & - & - & - & - & - & - & - & - & - & - & - & - & - & - & 65.4 & 65.6 & 65.6 & 67.6 & 67.5 & 70.1 & 74.2 & 76.5 & 77.5 & 75.7 & 74.6 \\
\hline & o & o & o & 0 & o & o & o & o & o & o & o & o & 0 & 1 & o & o & o & o & o & 2 & o & o & 5 & o & o \\
\hline \multirow[t]{3}{*}{ Turkey } & 4.5 & 4.5 & 4.5 & 4.5 & 4.5 & 4 & 3.5 & 4 & 4 & 3.5 & 4 & 3.5 & 3 & 3 & 3.5 & 4 & 3.5 & $\begin{array}{l}3.5 \\
\end{array}$ & 3 & 3 & 3 & 3.5 & 3.5 & 4 & 4.5 \\
\hline & - & - & - & - & - & - & - & - & - & - & - & - & - & - & 74.4 & 74.9 & 75.4 & 78.2 & 77.1 & 74.9 & 76.6 & 75.9 & 74.1 & 74.6 & 77.3 \\
\hline & 1 & o & 2 & 3 & 1 & 0 & 4 & 4 & o & o & o & o & 2 & 1 & 1 & 0 & o & 0 & 0 & 2 & 2 & 3 & 1 & o & o \\
\hline \multirow[t]{3}{*}{ Turkmenistan } & 1.5 & 1 & 1.5 & 2 & 2 & 2 & 2.5 & 2.5 & 2.5 & 2.5 & 2.5 & 3 & 3 & 3 & 3 & 2.5 & 2.5 & 2.5 & 3 & 3 & 3 & 3 & 3 & 3.5 & 3.5 \\
\hline & - & - & - & - & - & - & - & - & - & - & - & - & - & - & 86.1 & 87.5 & 86.2 & 84.3 & 82.5 & 79.7 & 77.4 & 76.7 & 78.2 & 77.4 & 76 \\
\hline & o & o & o & 1 & o & o & o & o & o & o & o & o & o & o & o & o & o & o & o & o & o & o & o & o & o \\
\hline \multirow[t]{3}{*}{ Uganda } & 3 & 3 & 3 & 3.5 & 3.5 & 3.5 & 3.5 & 3.5 & 3.5 & 3.5 & 4 & 4 & 4 & 4.5 & 4 & 4 & 3.5 & 3.5 & 3.5 & 3.5 & 3 & 3 & 3 & 2.5 & 3 \\
\hline & - & - & - & - & - & - & - & - & - & - & - & - & - & - & 94.5 & 96.4 & 96.1 & 96.9 & 97.5 & 96.3 & 96.5 & 96.6 & 96 & 97 & 97.7 \\
\hline & o & o & o & 3 & 1 & o & 2 & o & o & 1 & o & 1 & 1 & o & o & o & o & o & o & o & o & o & o & o & 2 \\
\hline \multirow[t]{3}{*}{ Ukraine } & 1.5 & 1.5 & 2.5 & 2 & 2 & 2 & 3 & 3 & 2.5 & 2.5 & 3 & 3 & 3 & 2.5 & 3 & 3 & 3 & 3 & 2.5 & 3 & 3 & 3 & 3.5 & 4.5 & 3.5 \\
\hline & - & - & - & - & - & - & - & - & - & - & - & - & - & - & 72.9 & 71.4 & 70.8 & 69.7 & 69.5 & 69 & 67.2 & 65.9 & 67.2 & 76.3 & 75.5 \\
\hline & o & 1 & o & o & o & o & o & 1 & o & 1 & o & o & o & o & o & o & o & o & 1 & o & o & 1 & 5 & o & o \\
\hline \multirow{3}{*}{$\begin{array}{l}\text { United Arab } \\
\text { Emirates }\end{array}$} & 1.5 & 1 & 1 & 1.5 & 1.5 & 2 & 2 & 1.5 & 1.5 & 2 & 2.5 & 2 & 2 & 2 & 2 & 2 & 2 & 1.5 & 1.5 & 1.5 & 2 & 3 & 2.5 & 3 & 2.5 \\
\hline & - & - & - & - & - & - & - & - & - & - & - & - & - & - & - & 51.6 & 51.2 & 51.8 & 52.4 & 50.4 & 48.9 & 47.3 & 47.6 & 46.2 & 44.5 \\
\hline & o & o & o & 1 & o & o & 1 & o & o & o & o & 2 & o & o & o & o & o & o & o & o & o & o & o & o & o \\
\hline \multirow[t]{3}{*}{ United Kingdom } & 1.5 & 1 & 1 & 1 & 1 & 1 & 2 & 1 & 1.5 & 2 & 2 & 2 & 2 & 2.5 & 2 & 2 & 2 & 2 & 1 & 1 & 1 & 1 & 1 & 1 & 1.5 \\
\hline & - & - & - & - & - & - & - & - & - & - & - & - & - & - & 34.2 & 34.1 & 32.9 & 33.6 & 33.9 & 34.1 & 35.3 & 33.2 & 34.3 & 33.4 & 32.4 \\
\hline & 4 & o & o & 1 & o & 2 & 5 & 2 & o & 2 & 2 & 2 & 2 & 2 & 2 & 1 & o & 3 & o & o & o & o & o & o & o \\
\hline
\end{tabular}




\begin{tabular}{|c|c|c|c|c|c|c|c|c|c|c|c|c|c|c|c|c|c|c|c|c|c|c|c|c|c|}
\hline & 1992 & 1993 & 1994 & 1995 & 1996 & 1997 & 1998 & 1999 & $\begin{array}{c}200 \\
\mathbf{0}\end{array}$ & $\begin{array}{c}200 \\
1\end{array}$ & $\begin{array}{c}200 \\
2\end{array}$ & $\begin{array}{c}\mathbf{2 0 0} \\
3\end{array}$ & $\begin{array}{c}200 \\
4\end{array}$ & $\begin{array}{c}200 \\
5\end{array}$ & $\underset{6}{200}$ & $\begin{array}{c}200 \\
7\end{array}$ & $\begin{array}{c}\mathbf{2 0 0} \\
8\end{array}$ & $\begin{array}{c}200 \\
9\end{array}$ & $\begin{array}{c}201 \\
0\end{array}$ & 2011 & 2012 & 2013 & 2014 & 2015 & 2016 \\
\hline \multirow[t]{3}{*}{ United States } & 2 & 1 & 1 & 1 & 1 & 1 & 1 & 1 & 1 & 1 & 2 & 2 & 3 & 3 & 3 & 3 & 3 & 3 & 3 & 3 & 3 & 2 & 2 & 2.5 & 2.5 \\
\hline & - & - & - & - & - & - & - & - & - & - & - & - & - & - & 34.5 & 33.6 & 32.8 & 34 & 35.3 & 34.8 & 34.8 & 33.5 & 35.4 & 35.4 & 34 \\
\hline & 1 & o & o & 1 & o & o & 2 & o & o & 1 & o & 1 & 1 & o & o & o & 1 & 1 & o & o & o & 1 & o & o & o \\
\hline \multirow[t]{3}{*}{ Uruguay } & 2 & 1.5 & 2 & 2 & 1 & 1.5 & 1.5 & 1.5 & 2 & 1 & 2 & 1.5 & 1.5 & 1 & 1 & 1 & 1 & 1 & 1 & 1 & 1 & 1 & 1 & 1 & 1 \\
\hline & - & - & - & - & - & - & - & - & - & - & - & - & - & - & 41.2 & 40.9 & 41.4 & 41.2 & 41.3 & 40.5 & 38.4 & 37.9 & 36.4 & 36.2 & 40.4 \\
\hline & 1 & o & o & o & o & o & o & o & o & o & o & o & o & o & o & o & o & o & o & o & o & o & o & o & o \\
\hline \multirow[t]{3}{*}{ Uzbekistan } & 1.5 & 2 & 2 & 2 & 2 & 2.5 & 2.5 & 2.5 & 2.5 & 3.5 & 3.5 & 3.5 & 3 & 4 & 3 & 3 & 3 & 3 & 3 & 3 & 3 & 3 & 3.5 & 3.5 & 3.5 \\
\hline & - & - & - & - & - & - & - & - & - & - & - & - & - & - & 94.4 & 93.5 & 93.4 & 92.8 & 90.5 & 88.3 & 87.5 & 86.9 & 86.3 & 85.3 & 83.5 \\
\hline & o & o & o & 1 & o & 2 & o & o & 1 & o & 1 & o & 2 & o & o & o & o & o & o & o & o & 3 & 1 & 1 & o \\
\hline \multirow[t]{3}{*}{ Venezuela } & 3.5 & 3.5 & 4 & 3.5 & 4.5 & 3.5 & 3 & 3 & 2.5 & 3 & 4 & 3 & 3 & 3 & 3.5 & 3.5 & 3.5 & 3.5 & 3 & 3 & 3.5 & 3 & 3.5 & 3.5 & 3.5 \\
\hline & - & - & - & - & - & - & - & - & - & - & - & - & - & - & 81.2 & 79.8 & 77.9 & 79.5 & 78.7 & 78.2 & 77.3 & 75.3 & 76.7 & 78.7 & 81.6 \\
\hline & 1 & o & o & o & o & 1 & 1 & o & o & 1 & 3 & 1 & o & o & o & o & o & 1 & o & o & o & o & o & o & o \\
\hline \multirow[t]{3}{*}{ Vietnam } & 2.5 & 2 & 2.5 & 2 & 2 & 2.5 & 2 & 2 & 2.5 & 2.5 & 2.5 & 3 & 3 & 3 & 2.5 & 2.5 & 3 & 2.5 & 2.5 & 2.5 & 3 & 3 & 3 & 3 & 3 \\
\hline & - & - & - & - & - & - & - & - & - & - & - & - & - & - & 78.6 & 77.8 & 74.6 & 76.9 & 76.6 & 76.1 & 74 & 73.1 & 72.7 & 72.4 & 70.7 \\
\hline & o & o & o & o & o & o & o & o & o & 1 & o & 1 & o & o & o & o & o & o & o & o & o & o & o & o & o \\
\hline \multirow[t]{3}{*}{ Yemen } & 3 & 3 & 4 & 3 & 2.5 & 3 & 3 & 2.5 & 3 & 3 & 3 & 3 & 4 & 4 & 3 & 4 & 4 & 4 & 4.5 & 4.5 & 3.5 & 3.5 & 4.5 & 5 & 5 \\
\hline & - & - & - & - & - & - & - & - & - & - & - & - & - & - & 96.6 & 93.2 & 95.4 & 98.1 & 100 & 100. & 104. & 107 & 105.4 & 108.2 & 111.5 \\
\hline & o & o & 1 & o & o & 1 & 2 & o & 2 & o & 2 & o & o & o & 1 & 2 & 1 & o & o & & & 1 & 2 & 3 & o \\
\hline \multirow[t]{3}{*}{ Yugoslavia } & 4 & 4 & 5 & 4.5 & 3 & 4 & 4.5 & 5 & 4 & 4 & 2.5 & 3 & 2.5 & 2.5 & 2.5 & - & - & - & - & - & - & - & - & - & - \\
\hline & - & - & - & - & - & - & - & - & - & - & - & - & - & - & - & - & - & - & - & - & - & - & - & - & - \\
\hline & 1 & o & o & o & o & o & o & o & o & o & o & o & o & o & o & o & o & o & o & o & o & o & o & o & o \\
\hline \multirow[t]{3}{*}{ Zambia } & 2.5 & 3 & 2.5 & 2.5 & 2.5 & 3 & 3.5 & 2 & 2.5 & 3 & 3 & 3 & 3 & 3 & 3 & 2.5 & 3 & 3 & 4 & 3 & 3 & 3 & 3 & 2.5 & 3 \\
\hline & - & - & - & - & - & - & - & - & - & - & - & - & - & - & 79.6 & 80.6 & 81.6 & 84.2 & 83.9 & 83.8 & 85.9 & 86.6 & 86.2 & 85.1 & 86.3 \\
\hline & o & o & o & o & o & o & 1 & o & o & o & o & o & o & o & o & o & o & o & o & o & o & o & o & o & o \\
\hline
\end{tabular}




\begin{tabular}{|c|c|c|c|c|c|c|c|c|c|c|c|c|c|c|c|c|c|c|c|c|c|c|c|c|c|}
\hline & 1992 & 1993 & 1994 & 1995 & 1996 & 1997 & 1998 & 1999 & $\begin{array}{c}200 \\
\mathbf{0}\end{array}$ & $\begin{array}{c}200 \\
1\end{array}$ & $\begin{array}{c}200 \\
2\end{array}$ & $\begin{array}{c}200 \\
3\end{array}$ & $\begin{array}{c}200 \\
4\end{array}$ & $\begin{array}{c}200 \\
5\end{array}$ & $\underset{6}{200}$ & $\begin{array}{c}200 \\
7\end{array}$ & $\begin{array}{c}200 \\
8\end{array}$ & $\begin{array}{c}200 \\
9\end{array}$ & $\begin{array}{c}201 \\
\mathbf{0}\end{array}$ & 2011 & 2012 & 2013 & 2014 & 2015 & 2016 \\
\hline \multirow[t]{3}{*}{ Zimbabwe } & 2 & 2 & 2 & 2 & 3 & 2 & 2.5 & 3 & 3.5 & 3.5 & 3.5 & 4 & 3.5 & 4 & 4 & 4 & 4.5 & 3.5 & 3.5 & 3.5 & 3.5 & 3 & 3 & 3.5 & 3.5 \\
\hline & - & - & - & - & - & - & - & - & - & - & - & - & - & - & 108. & 110.1 & 112.5 & 114.1 & 110.2 & 107.9 & 106.3 & 105.2 & 102.8 & 100 & 100.5 \\
\hline & o & o & o & o & o & o & o & o & o & o & o & o & o & o & 9 & o & o & o & o & o & o & o & o & o & o \\
\hline
\end{tabular}




\section{Appendix B}

\begin{tabular}{|c|c|c|c|c|c|c|}
\hline VEO Name & Type & Emerged & Ended & Countries & Type of Attack & Target Type \\
\hline o55 Brigade/Lashkar-al-Zil & Religious & 1995 & 2001 & Afghanistan, Pakistan & $\begin{array}{l}\text { Armed Assault, Bombing/Explosion, } \\
\text { Unarmed Assault }\end{array}$ & Military \\
\hline 11-9 the Second & Religious & 2005 & - & Jordan & Assassination, Bombing/Explosion & Businesses \\
\hline 14 March Coalition & Nationalist & 2005 & 2005 & Lebanon & Armed Assault & Terrorists/Non-State Militia \\
\hline 1920 Revolution Brigades & Religious & 2003 & 2004 & Iraq & Bombing/Explosion & $\begin{array}{l}\text { Journalists \& Media, } \\
\text { Telecommunication }\end{array}$ \\
\hline 21 June & Left-Wing & 2003 & 2003 & Greece & Bombing/Explosion & Businesses \\
\hline $\begin{array}{l}23 \text { May Democratic Alliance for Change } \\
\text { (ADC) }\end{array}$ & Nationalist & 2006 & - & Algeria, Mali, Somalia & Armed Assault, Hostage Taking & $\begin{array}{l}\text { Businesses, Government (General), } \\
\text { Military, Tourists }\end{array}$ \\
\hline 969 Movement/Wirathu & Religious & 2001 & - & Myanmar, Burma & $\begin{array}{l}\text { Armed Assault, Bombing/Explosion, } \\
\text { Unarmed Assault }\end{array}$ & $\begin{array}{l}\text { Private Citizens \& Property, Religious } \\
\text { Figures/Institutions }\end{array}$ \\
\hline Abdullah Azzam Brigades (AAB) & Religious & 2004 & - & Egypt, Jordan, Lebanon, Syria & $\begin{array}{l}\text { Armed Assault, Assassination, } \\
\text { Bombing/Explosion }\end{array}$ & $\begin{array}{l}\text { Businesses, Government (Diplomatic), } \\
\text { Government (General), Maritime, } \\
\text { Military, Private Citizens \& Property, } \\
\text { Terrorists/Non-State Militia, Tourists, } \\
\text { Utilities }\end{array}$ \\
\hline Abdurajak Janjalani Brigade & Religious & 1999 & 1999 & Philippines & Armed Assault & Private Citizens \& Property \\
\hline Abis Brigade & Religious & 2014 & - & Bahrain & Bombing/Explosion & Businesses, Police \\
\hline Abkhaz Forces & Nationalist & 2001 & - & Russia & Armed Assault & $\begin{array}{l}\text { Government (General), Military, Private } \\
\text { Citizens \& Property }\end{array}$ \\
\hline Abu al-Abbas & Religious & 2004 & 2004 & Iraq & Hostage Taking & Journalists \& Media \\
\hline Abu al-Fadl al-Abbas Forces & Religious & 2014 & - & Iraq & Armed Assault & Terrorists/Non-State Militia \\
\hline Abu al-Rish Brigades & Nationalist & 1993 & - & Israel & Hostage Taking & Private Citizens \& Property \\
\hline $\begin{array}{l}\text { Abu Bakr al-Siddiq Fundamentalist } \\
\text { Brigades (ABSB) }\end{array}$ & Religious & 2004 & - & Iraq & Bombing/Explosion, Hostage Taking & $\begin{array}{l}\text { Government (General), NGO, Private } \\
\text { Citizens \& Property }\end{array}$ \\
\hline Abu Hafs al-Masri Brigade & Religious & 2003 & 2005 & Iraq, Spain, United Kingdom & Bombing/Explosion & $\begin{array}{l}\text { Businesses, Private Citizens \& Property, } \\
\text { Transportation, Utilities }\end{array}$ \\
\hline Abu Hanif's Jamaat & Religious & 2013 & - & Iraq, Russia, Syria, Turkey & Armed Assault, Unarmed Assault & $\begin{array}{l}\text { Private Citizens \& Property, } \\
\text { Terrorists/Non-State Militia }\end{array}$ \\
\hline Abu Ja'afar al-Mansur Brigades & Religious & 2012 & - & Iraq & Bombing/Explosion & $\begin{array}{l}\text { Military, Private Citizens \& Property, } \\
\text { Terrorists/Non-State Militia }\end{array}$ \\
\hline Abu Mus'ab al-Zarqawi Battalion & Religious & 2006 & 2006 & Iraq, Lebanon, Saudi Arabia & Bombing/Explosion & Government (Diplomatic) \\
\hline Abu Nayaf al-Afghani & Religious & 2004 & - & Spain & Bombing/Explosion & $\begin{array}{l}\text { Private Citizens \& Property, } \\
\text { Transportation }\end{array}$ \\
\hline $\begin{array}{l}\text { Abu Omar Network- West Indonesia } \\
\text { Mujahideen }\end{array}$ & Nationalist & 2010 & - & Indonesia & Armed Assault, Bombing/Explosion & Government (General) \\
\hline
\end{tabular}




\begin{tabular}{|c|c|c|c|c|c|c|}
\hline VEO Name & Type & Emerged & Ended & Countries & Type of Attack & Target Type \\
\hline Abu Salim Martyr's Brigade & Religious & 2012 & - & Libya & $\begin{array}{l}\text { Armed Assault, Assassination, } \\
\text { Bombing/Explosion, Hostage Taking }\end{array}$ & $\begin{array}{l}\text { Government (Diplomatic), Government } \\
\text { (General), Private Citizens \& Property, } \\
\text { Terrorists/Non-State Militia }\end{array}$ \\
\hline Accolta Nazinuale Corsa & Nationalist & 2002 & 2003 & France & Armed Assault & Businesses \\
\hline Aceh Transition Committee (KPA) & Nationalist & 2005 & - & Indonesia, Malaysia & Armed Assault & Government (General), Military \\
\hline A'chik Matgrik Elite Force (AMEF) & Nationalist & 2015 & - & India & Bombing/Explosion, Hostage Taking & Businesses, Private Citizens \& Property \\
\hline $\begin{array}{l}\text { A'chik National Cooperative Army } \\
\text { (ANCA) }\end{array}$ & Nationalist & 2014 & & India & $\begin{array}{l}\text { Armed Assault, Bombing/Explosion, } \\
\text { Hostage Taking }\end{array}$ & $\begin{array}{l}\text { Educational Institutes, Police, Private } \\
\text { Citizens \& Property, Transportation }\end{array}$ \\
\hline $\begin{array}{l}\text { A'chik National Liberation Army } \\
\text { (ANLA) }\end{array}$ & Nationalist & 2014 & - & India & Hostage Taking & Businesses, Private Citizens \& Property \\
\hline $\begin{array}{l}\text { A'chik National Volunteer Council } \\
\text { (ANVC)/A'chik National Volunteer } \\
\text { Council-B (ANVC-B) }\end{array}$ & Nationalist & 1995 & - & Bangladesh, India, Myanmar, Burma & $\begin{array}{l}\text { Armed Assault, Assassination, } \\
\text { Bombing/Explosion, } \\
\text { Facility/Infrastructure Attack, Hostage } \\
\text { Taking }\end{array}$ & $\begin{array}{l}\text { Businesses, Military, Police, Private } \\
\text { Citizens \& Property, Transportation }\end{array}$ \\
\hline $\begin{array}{l}\text { A'chik Songna An'pachakgipa Kotok } \\
\text { (ASAK) }\end{array}$ & Nationalist & 2014 & & India & Bombing/Explosion, Hostage Taking & $\begin{array}{l}\text { Businesses, Educational Institutes, } \\
\text { Government (General), Police, Private } \\
\text { Citizens \& Property, Transportation }\end{array}$ \\
\hline $\begin{array}{l}\text { Actiefront Nationistisch Nederland } \\
\text { (Action Front Nationalist Netherlands) }\end{array}$ & $\begin{array}{l}\text { Right- } \\
\text { Wing }\end{array}$ & 1992 & 1992 & Netherlands & Bombing/Explosion & $\begin{array}{l}\text { Government (General), Journalists \& } \\
\text { Media, Private Citizens \& Property }\end{array}$ \\
\hline $\begin{array}{l}\text { Action Committee of Winegrowers } \\
\text { (CAV) }\end{array}$ & Left-Wing & 1999 & 2002 & France & Bombing/Explosion & Transportation \\
\hline Action Group Extreme Beate & Left-Wing & 2005 & 2005 & Denmark & Bombing/Explosion & Government (General) \\
\hline Adan Abyan Islamic Army (AAIA) & Religious & 1994 & 2003 & Yemen & $\begin{array}{l}\text { Armed Assault, Bombing/Explosion, } \\
\text { Hostage Taking }\end{array}$ & $\begin{array}{l}\text { Airports \& Aircraft, Businesses, } \\
\text { Government (Diplomatic), Maritime, } \\
\text { Military, Private Citizens \& Property, } \\
\text { Religious Figures/Institutions, Tourists, } \\
\text { Utilities }\end{array}$ \\
\hline $\begin{array}{l}\text { Adivasi Cobra Force/Adivasi Cobra } \\
\text { Militants of Assam (ACMA) }\end{array}$ & Nationalist & 1996 & & India & $\begin{array}{l}\text { Armed Assault, Assassination, } \\
\text { Bombing/Explosion, } \\
\text { Facility/Infrastructure Attack, Hostage } \\
\text { Taking }\end{array}$ & $\begin{array}{l}\text { Airports \& Aircraft, Educational } \\
\text { Institutes, Government (General), } \\
\text { Military, Police, Private Citizens \& } \\
\text { Property, Religious Figures/Institutions, } \\
\text { Terrorists/Non-State Militia, Utilities }\end{array}$ \\
\hline $\begin{array}{l}\text { Adivasi National Liberation Army } \\
\text { (ANLA) }\end{array}$ & Nationalist & 2007 & - & India & $\begin{array}{l}\text { Armed Assault, Bombing/Explosion, } \\
\text { Hostage Taking }\end{array}$ & $\begin{array}{l}\text { Businesses, Educational Institutes, } \\
\text { Private Citizens \& Property, } \\
\text { Transportation }\end{array}$ \\
\hline Adivasi People's Army (APA) & Left-Wing & 2011 & - & India & Armed Assault, Bombing/Explosion & Government (General), Transportation \\
\hline Adivasi Security Force & Nationalist & 2008 & - & Bangladesh, India & Armed Assault, Bombing/Explosion & $\begin{array}{l}\text { Private Citizens \& Property, } \\
\text { Transportation }\end{array}$ \\
\hline
\end{tabular}




\begin{tabular}{|c|c|c|c|c|c|c|}
\hline VEO Name & Type & Emerged & Ended & Countries & Type of Attack & Target Type \\
\hline Ahfad al-Rasul Brigade & Nationalist & 2012 & - & Syria & Armed Assault, Bombing/Explosion & Military, Terrorists/Non-State Militia \\
\hline Ahle Sunnat Wal Jammaat (ASWJ) & Religious & 2002 & - & Pakistan, Somalia & Armed Assault, Assassination & $\begin{array}{l}\text { Private Citizens \& Property, Religious } \\
\text { Figures/Institutions }\end{array}$ \\
\hline $\begin{array}{l}\text { Ahle-Hadith Andolan Bangladesh } \\
\text { (AHAB) }\end{array}$ & Religious & 1995 & - & Bangladesh & Bombing/Explosion & Unknown \\
\hline Ahlu-Sunah Wal-Jamea & Religious & 2008 & - & Somalia & $\begin{array}{l}\text { Armed Assault, Assassination, } \\
\text { Bombing/Explosion, Hostage Taking }\end{array}$ & $\begin{array}{l}\text { Government (General), Journalists \& } \\
\text { Media, Military, Private Citizens \& } \\
\text { Property, Terrorists/Non-State Militia }\end{array}$ \\
\hline $\begin{array}{l}\text { Ahlu-sunnah Wa-Jamo/Ansar al-Sunna } \\
\text { (Shabaab) }\end{array}$ & Religious & 2015 & - & Mozambique & Armed Assault & Businesses, Government (General) \\
\hline Ahrar al-JalilFree People of Galilee & Nationalist & 2003 & 2003 & Israel & $\begin{array}{l}\text { Assassination, Bombing/Explosion, } \\
\text { Hostage Taking, Unarmed Assault }\end{array}$ & $\begin{array}{l}\text { Military, Private Citizens \& Property, } \\
\text { Religious Figures/Institutions }\end{array}$ \\
\hline $\begin{array}{l}\text { Ahrar al-Sham/Harakat Ahrar al-Sham } \\
\text { al-Islamiyya }\end{array}$ & Religious & 2012 & - & Syria & $\begin{array}{l}\text { Armed Assault, Assassination, } \\
\text { Bombing/Explosion, Hostage Taking }\end{array}$ & $\begin{array}{l}\text { Military, Private Citizens \& Property, } \\
\text { Terrorists/Non-State Militia }\end{array}$ \\
\hline Ahrar-ul-Hind & Religious & 2014 & - & Pakistan & Armed Assault, Bombing/Explosion & Government (General) \\
\hline Ahwaz Liberation Organization (ALO) & Religious & 2013 & - & Iran & Bombing/Explosion & Utilities \\
\hline Aidid Rebels & $\begin{array}{l}\text { Right- } \\
\text { Wing }\end{array}$ & 1992 & 1992 & Somalia & Hijacking & Maritime \\
\hline Ajnad al-Kavkaz & Religious & 2015 & - & Russia, Syria & Armed Assault & Government (General) \\
\hline Ajnad al-Sham Islamic Union & Religious & 2013 & - & Syria & Bombing/Explosion & Private Citizens \& Property \\
\hline Ajnad Misr & Religious & 2013 & - & Egypt & Assassination, Bombing/Explosion & $\begin{array}{l}\text { Businesses, Educational Institutes, } \\
\text { Government (General), Journalists \& } \\
\text { Media, Police, Private Citizens \& } \\
\text { Property, Transportation }\end{array}$ \\
\hline Akhil Krantikari & Left-Wing & 2004 & - & Nepal & Bombing/Explosion & Educational Institutes \\
\hline al-Abbas Brigade & Nationalist & 2011 & - & Iraq, Syria & Armed Assault & Unknown \\
\hline al-Ahrar Al Jalil & Nationalist & 2003 & - & Israel, Palestinian State & $\begin{array}{l}\text { Armed Assault, Bombing/Explosion, } \\
\text { Hostage Taking }\end{array}$ & Military, Private Citizens \& Property \\
\hline al-Ahwal Brigades & Religious & 2004 & - & Iraq & Bombing/Explosion, Hostage Taking & Military \\
\hline $\begin{array}{l}\text { al-Ahwaz Arab Popular Democratic } \\
\text { Front (APDF)/al-Ahwaz Arab People's } \\
\text { Democratic Front (APDF) }\end{array}$ & Nationalist & 2005 & - & Iran, United Kingdom & Bombing/Explosion & $\begin{array}{l}\text { Businesses, Government (General), } \\
\text { Private Citizens \& Property, Religious } \\
\text { Figures/Institutions }\end{array}$ \\
\hline al-Ansar Brigades (AIM) & Nationalist & 2012 & - & Syria & Armed Assault, Bombing/Explosion & Government (General), Military \\
\hline
\end{tabular}




\begin{tabular}{|c|c|c|c|c|c|c|}
\hline VEO Name & Type & Emerged & Ended & Countries & Type of Attack & Target Type \\
\hline Al-Aqsa Martyrs Brigade (AAMB) & Nationalist & 2000 & - & Iran, Israel, Palestinian State & $\begin{array}{l}\text { Armed Assault, Assassination, } \\
\text { Bombing/Explosion, } \\
\text { Facility/Infrastructure Attack, Hostage } \\
\text { Taking }\end{array}$ & $\begin{array}{l}\text { Businesses, Educational Institutes, } \\
\text { Government (Diplomatic), Government } \\
\text { (General), Journalists \& Media, Military, } \\
\text { Police, Private Citizens \& Property, } \\
\text { Religious Figures/Institutions, } \\
\text { Transportation }\end{array}$ \\
\hline al-Arifeen & Religious & 2001 & - & Pakistan & $\begin{array}{l}\text { Armed Assault, Assassination, } \\
\text { Bombing/Explosion }\end{array}$ & Businesses, Government (General) \\
\hline al-Ashtar Brigades - Bahrain & Religious & 2013 & - & Bahrain & Bombing/Explosion & $\begin{array}{l}\text { Government (General), Military, Police, } \\
\text { Private Citizens \& Property, Religious } \\
\text { Figures/Institutions }\end{array}$ \\
\hline al-Bara' ibn Malik Martyrs Brigade & Nationalist & 2005 & - & Iraq, Jordan, Syria & Bombing/Explosion, Hostage Taking & $\begin{array}{l}\text { Government (General), Military, } \\
\text { Tourists }\end{array}$ \\
\hline $\begin{array}{l}\text { Alexandros Grigoropoulos Anarchist } \\
\text { Attack Group }\end{array}$ & Left-Wing & 2009 & - & Greece & Facility/Infrastructure Attack & Police \\
\hline al-Fajr Islamic Movement & Nationalist & 2012 & - & Syria & Assassination, Bombing/Explosion & Government (General), Military \\
\hline al-Faran & Nationalist & 1995 & 1995 & India & Hijacking, Hostage Taking & Tourists, Transportation \\
\hline al-Faruq Brigades & Religious & 2003 & - & Iraq & Bombing/Explosion & Military \\
\hline al-Fath al-Mubin Brigades & Nationalist & 2006 & - & Palestinian State & Bombing/Explosion & Private Citizens \& Property \\
\hline al-Farooq & Religious & 1998 & 1998 & Afghanistan, Pakistan & Armed Assault & Government (Diplomatic) \\
\hline al-Furqan Brigades & Religious & 2013 & - & Egypt, Syria & Armed Assault, Bombing/Explosion & $\begin{array}{l}\text { Maritime, Private Citizens \& Property, } \\
\text { Telecommunication, Utilities }\end{array}$ \\
\hline al-Fursan Brigades & Nationalist & 2005 & - & Iraq & Hostage Taking & Private Citizens \& Property \\
\hline al-Gamaa al-Jihadiyah & Nationalist & 2012 & - & Egypt & Armed Assault, Bombing/Explosion & Government (General) \\
\hline Algerian Mujahideen for Muslems & Religious & 1992 & 1992 & Algeria & Armed Assault & Government (General) \\
\hline al-Ghurabaa & Religious & 2004 & - & United Kingdom & Armed Assault & Journalists \& Media \\
\hline al-Hadid & Nationalist & 1994 & 1994 & India & Hostage Taking & $\begin{array}{l}\text { Educational Institutes, Private Citizens } \\
\text { \& Property, Tourists }\end{array}$ \\
\hline al-Hamas Mujahideen & Religious & 2001 & 2001 & India & Assassination & Government (General) \\
\hline al-Hamza Brigades & Nationalist & 2012 & - & Afghanistan & Armed Assault, Bombing/Explosion & Government (General), Military, Police \\
\hline $\begin{array}{l}\text { al-Haramain Brigades/al-Haramayn } \\
\text { Brigades/Islamic Movement for Change }\end{array}$ & Religious & 1995 & 2004 & Saudi Arabia, Syria & Assassination, Bombing/Explosion & $\begin{array}{l}\text { Government (General), Police, Religious } \\
\text { Figures/Institutions, Transportation }\end{array}$ \\
\hline al-Herak al-Janoubi & Nationalist & 2007 & - & Yemen & Armed Assault, Assassination & $\begin{array}{l}\text { Government (General), Terrorists/Non- } \\
\text { State Militia }\end{array}$ \\
\hline al-Hijratain Foundation & Religious & 2008 & - & Sudan & Armed Assault, Assassination & Government (Diplomatic) \\
\hline Ali bin Abu Talib Jihad Organization & Religious & 2005 & - & Iraq & Hostage Taking & Private Citizens \& Property \\
\hline al-Imam Ali Brigades & Nationalist & 2006 & - & Iraq & Hostage Taking & Private Citizens \& Property \\
\hline
\end{tabular}




\begin{tabular}{|c|c|c|c|c|c|c|}
\hline VEO Name & Type & Emerged & Ended & Countries & Type of Attack & Target Type \\
\hline al-Imam Army Cell & Nationalist & 2013 & - & Bahrain, Iran & Armed Assault, Bombing/Explosion & Military, Private Citizens \& Property \\
\hline al-Intiqami al-Pakistani & Religious & 2002 & 2002 & Pakistan & Armed Assault, Bombing/Explosion & Businesses, Educational Institutes \\
\hline al-Islambouli Brigades of al-Qaeda & Nationalist & 1995 & - & Egypt, Pakistan, Russia & $\begin{array}{l}\text { Assassination, Bombing/Explosion, } \\
\text { Hijacking }\end{array}$ & $\begin{array}{l}\text { Airports \& Aircraft, Government } \\
\text { (Diplomatic), Government (General), } \\
\text { Transportation }\end{array}$ \\
\hline al-Ittihad al-Islami (AIAI) & Religious & 1992 & 2005 & Ethiopia, Somalia & $\begin{array}{l}\text { Armed Assault, Assassination, } \\
\text { Bombing/Explosion }\end{array}$ & $\begin{array}{l}\text { Businesses, Government (General), } \\
\text { NGO, Religious Figures/Institutions, } \\
\text { Transportation }\end{array}$ \\
\hline $\begin{array}{l}\text { al-Jama'ah al-Islamiya al-Musallaha } \\
\text { (HIJM) }\end{array}$ & Religious & 1992 & 2004 & Algeria & $\begin{array}{l}\text { Assassination, Bombing/Explosion, } \\
\text { Hostage Taking, Unarmed Assault }\end{array}$ & $\begin{array}{l}\text { Government (General), Private Citizens } \\
\text { \& Property }\end{array}$ \\
\hline al-Jamas & Religious & 2012 & - & China & Bombing/Explosion & Private Citizens \& Property \\
\hline al-Jihad al-Alami & Religious & 2010 & - & Afghanistan, Iraq, Norway & Bombing/Explosion & Unknown \\
\hline al-Kafn al-Abyad & Religious & 2014 & - & Syria & $\begin{array}{l}\text { Armed Assault, Assassination, } \\
\text { Bombing/Explosion }\end{array}$ & $\begin{array}{l}\text { Government (General), Terrorists/Non- } \\
\text { State Militia, Violent Political Party }\end{array}$ \\
\hline al-Kobar & Religious & 2006 & - & Philippines & Bombing/Explosion & $\begin{array}{l}\text { Businesses, Private Citizens \& Property, } \\
\text { Transportation, Utilities }\end{array}$ \\
\hline All Germanic Heathens Front & Religious & 2001 & 2006 & Norway & Unknown & Unknown \\
\hline All India Sikh Students Federation & Religious & 1995 & 1995 & India & Bombing/Explosion & Police \\
\hline All Kamatapur Liberation Force & Nationalist & 2008 & - & India & Hostage Taking & Businesses, Private Citizens \& Property \\
\hline Allahr Dal & Religious & 2009 & - & Bangladesh & Bombing/Explosion & Private Citizens \& Property \\
\hline Alliance for Freedom and Democracy & Nationalist & 2006 & - & Ethiopia & Unknown & Government (General) \\
\hline $\begin{array}{l}\text { Alliance of Democratic Forces for the } \\
\text { Liberation of Congo-Zaire (ADFL) }\end{array}$ & Nationalist & 1998 & - & Congo, Democratic Republic & Armed Assault, Bombing/Explosion & Private Citizens \& Property \\
\hline Alliance of Eritrean National Force & Nationalist & 1999 & - & Eritrea, Saudi Arabia, Sudan & Armed Assault & Military \\
\hline $\begin{array}{l}\text { Alliance of Patriots for a Free and } \\
\text { Sovereign Congo (APCLS) }\end{array}$ & Nationalist & 2006 & - & Congo, Democratic Republic, Rwanda & $\begin{array}{l}\text { Armed Assault, Hostage Taking, } \\
\text { Unarmed Assault }\end{array}$ & Military, Private Citizens \& Property \\
\hline Allied Democratic Forces (ADF) & $\begin{array}{l}\text { Right- } \\
\text { Wing }\end{array}$ & 1995 & - & $\begin{array}{l}\text { Congo, Democratic Republic, Kenya, } \\
\text { Sudan, Tanzania, Uganda }\end{array}$ & $\begin{array}{l}\text { Armed Assault, Assassination, } \\
\text { Bombing/Explosion, } \\
\text { Facility/Infrastructure Attack, Hostage } \\
\text { Taking }\end{array}$ & $\begin{array}{l}\text { Businesses, Educational Institutes, } \\
\text { Government (Diplomatic), Government } \\
\text { (General), Military, NGO, Police, Private } \\
\text { Citizens \& Property, Religious } \\
\text { Figures/Institutions, Transportation, } \\
\text { Utilities }\end{array}$ \\
\hline al-Madina & Nationalist & 2002 & - & India, Pakistan & Armed Assault, Bombing/Explosion & Government (General), Military, Police \\
\hline al-Majd Brigades & Nationalist & 2006 & - & Palestinian State & Bombing/Explosion & Private Citizens \& Property \\
\hline
\end{tabular}




\begin{tabular}{|c|c|c|c|c|c|c|}
\hline VEO Name & Type & Emerged & Ended & Countries & Type of Attack & $\begin{array}{c}\text { Target Type } \\
\end{array}$ \\
\hline al-Mansoorian & Nationalist & 2002 & 2007 & India, Pakistan & $\begin{array}{l}\text { Armed Assault, Assassination, } \\
\text { Bombing/Explosion, } \\
\text { Facility/Infrastructure Attack }\end{array}$ & $\begin{array}{l}\text { Businesses, Government (General), } \\
\text { Military, Police, Private Citizens \& } \\
\text { Property, Tourists }\end{array}$ \\
\hline al-Ma'unah & Nationalist & 2000 & 2000 & Malaysia & Armed Assault, Bombing/Explosion & Military, Religious Figures/Institutions \\
\hline al-Mudzahid & Religious & 1992 & 1995 & Bosnia and Herzegovina & Unknown & Unknown \\
\hline al-Mujahedeen al-Muhajereen & Religious & 2011 & - & Somalia, Yemen & Armed Assault, Bombing/Explosion & Unknown \\
\hline al-Mujahedin Special Unit (MSU) & Religious & 1992 & 1992 & Bosnia and Herzegovina & Armed Assault & Terrorists/Non-State Militia \\
\hline al-Mukhtar Group & $\begin{array}{l}\text { Right- } \\
\text { Wing }\end{array}$ & 2011 & - & Pakistan & Hostage Taking & Businesses, Private Citizens \& Property \\
\hline Al-Naqshabandiya Army & Religious & 2006 & - & Iraq & Armed Assault, Bombing/Explosion & $\begin{array}{l}\text { Businesses, Military, Police, Private } \\
\text { Citizens \& Property, Terrorists/Non- } \\
\text { State Militia }\end{array}$ \\
\hline al-Nasireen Group & Nationalist & 2005 & - & India, Pakistan & $\begin{array}{l}\text { Armed Assault, Bombing/Explosion, } \\
\text { Facility/Infrastructure Attack }\end{array}$ & $\begin{array}{l}\text { Military, Police, Private Citizens \& } \\
\text { Property, Tourists, Transportation }\end{array}$ \\
\hline al-Nawaz & Nationalist & 1999 & 2000 & Pakistan & Bombing/Explosion & $\begin{array}{l}\text { Businesses, Private Citizens \& Property, } \\
\text { Transportation }\end{array}$ \\
\hline al-Nusrah Front & Religious & 2012 & - & Iraq, Lebanon, Syria & $\begin{array}{l}\text { Armed Assault, Assassination, } \\
\text { Bombing/Explosion, Hostage Taking }\end{array}$ & $\begin{array}{l}\text { Businesses, Educational Institutes, } \\
\text { Government (Diplomatic), Government } \\
\text { (General), Journalists \& Media, Military, } \\
\text { Police, Private Citizens \& Property, } \\
\text { Religious Figures/Institutions, } \\
\text { Terrorists/Non-State Militia, Violent } \\
\text { Political Party }\end{array}$ \\
\hline al-Qaeda in Afghanistan & Religious & 2001 & - & Afghanistan, Pakistan & Assassination, Bombing/Explosion & $\begin{array}{l}\text { Airports \& Aircraft, Businesses, } \\
\text { Government (Diplomatic), Government } \\
\text { (General), Military, Private Citizens \& } \\
\text { Property, Religious Figures/Institutions, } \\
\text { Transportation }\end{array}$ \\
\hline al-Qaeda in Lebanon & Religious & 2006 & - & Lebanon & Bombing/Explosion & Military \\
\hline al-Qaeda in Levant and Egypt (AJ) & Religious & 2004 & - & Egypt, Jordan, Syria & Bombing/Explosion & Private Citizens \& Property, Tourists \\
\hline al-Qaeda in Libya (ASQL) & Religious & 2012 & - & Libya & Armed Assault, Bombing/Explosion & Military \\
\hline
\end{tabular}




\begin{tabular}{|c|c|c|c|c|c|c|}
\hline VEO Name & Type & Emerged & Ended & Countries & Type of Attack & Target Type \\
\hline $\begin{array}{l}\text { al-Qaeda in the Arabian Peninsula } \\
\text { (AQAP) }\end{array}$ & Religious & 2003 & - & $\begin{array}{l}\text { Saudi Arabia, the United Arab Emirates, } \\
\text { United States, Yemen }\end{array}$ & $\begin{array}{l}\text { Armed Assault, Assassination, } \\
\text { Bombing/Explosion, } \\
\text { Facility/Infrastructure Attack, } \\
\text { Hijacking, Hostage Taking }\end{array}$ & $\begin{array}{l}\text { Airports \& Aircraft, Businesses, } \\
\text { Educational Institutes, Government } \\
\text { (Diplomatic), Government (General), } \\
\text { Journalists \& Media, Maritime, Military, } \\
\text { NGO, Police, Private Citizens \& } \\
\text { Property, Religious Figures/Institutions, } \\
\text { Terrorists/Non-State Militia, Tourists, } \\
\text { Utilities, Violent Political Party }\end{array}$ \\
\hline $\begin{array}{l}\text { al-Qaeda in the Indian Subcontinent } \\
\text { (AQIS) }\end{array}$ & Religious & 2014 & - & India, Myanmar, Burma, Pakistan & $\begin{array}{l}\text { Armed Assault, Assassination, } \\
\text { Bombing/Explosion, Hostage Taking }\end{array}$ & $\begin{array}{l}\text { Educational Institutes, Government } \\
\text { (General), Journalists \& Media, Military, } \\
\text { Police, Private Citizens \& Property, } \\
\text { Terrorists/Non-State Militia }\end{array}$ \\
\hline $\begin{array}{l}\text { al-Qaeda in the Lands of the Islamic } \\
\text { Maghreb (AQIM)/Salafist Group for } \\
\text { Preaching and Fighting (GSPC) }\end{array}$ & Religious & 1998 & - & $\begin{array}{l}\text { Afghanistan, Algeria, Belgium, Burkina } \\
\text { Faso, Chad, France, Iran, Iraq, Italy, } \\
\text { Libya, Mali, Mauritania, Morocco, Niger, } \\
\text { Nigeria, Spain, Sudan, Syria, United } \\
\text { Arab Emirates, United States }\end{array}$ & $\begin{array}{l}\text { Armed Assault, Assassination, } \\
\text { Bombing/Explosion, } \\
\text { Facility/Infrastructure Attack, } \\
\text { Hijacking, Hostage Taking, Unarmed } \\
\text { Assault }\end{array}$ & $\begin{array}{l}\text { Airports \& Aircraft, Businesses, } \\
\text { Educational Institutes, Government } \\
\text { (Diplomatic), Government (General), } \\
\text { Journalists \& Media, Military, NGO, } \\
\text { Police, Private Citizens \& Property, } \\
\text { Religious Figures/Institutions, } \\
\text { Terrorists/Non-State Militia, Tourists, } \\
\text { Transportation, Utilities }\end{array}$ \\
\hline al-Qaeda in the Sinai Peninsular & Religious & 2011 & - & Egypt & Armed Assault, Bombing/Explosion & $\begin{array}{l}\text { Government (General), Private Citizens } \\
\text { \& Property, Religious } \\
\text { Figures/Institutions }\end{array}$ \\
\hline al-Qaeda in Tunisia & Religious & 2014 & - & Algeria, Tunisia & $\begin{array}{l}\text { Armed Assault, Bombing/Explosion, } \\
\text { Hostage Taking }\end{array}$ & $\begin{array}{l}\text { Government (Diplomatic), Government } \\
\text { (General) }\end{array}$ \\
\hline al-Qaeda in Yemen (AQY) & Religious & 2006 & - & Yemen & $\begin{array}{l}\text { Armed Assault, Assassination, } \\
\text { Bombing/Explosion }\end{array}$ & $\begin{array}{l}\text { Businesses, Government (Diplomatic), } \\
\text { Military, Police, Tourists, Utilities }\end{array}$ \\
\hline al-Qaeda Indonesia & Religious & 2009 & - & Indonesia & Armed Assault, Bombing/Explosion & $\begin{array}{l}\text { Government (General), Police, Private } \\
\text { Citizens \& Property, Religious } \\
\text { Figures/Institutions }\end{array}$ \\
\hline al-Qaeda Kurdish Battalions (AQKB) & Religious & 2007 & - & Iran, Iraq & Armed Assault, Bombing/Explosion & $\begin{array}{l}\text { Government (General), Private Citizens } \\
\text { \& Property }\end{array}$ \\
\hline $\begin{array}{l}\text { al-Qaeda Network for Southwestern } \\
\text { Khulna Division }\end{array}$ & Religious & 2006 & - & Bangladesh & Bombing/Explosion & Government (General) \\
\hline $\begin{array}{l}\text { al-Qaeda Organization for Jihad in } \\
\text { Sweden }\end{array}$ & Religious & 2005 & 2005 & Sweden & Facility/Infrastructure Attack & Government (General) \\
\hline
\end{tabular}




\begin{tabular}{|c|c|c|c|c|c|c|}
\hline VEO Name & Type & Emerged & Ended & Countries & Type of Attack & Target Type \\
\hline $\begin{array}{l}\text { al-Qaeda Organization in the Land of the } \\
\text { Two Rivers (AQI) }\end{array}$ & Religious & 2003 & - & $\begin{array}{l}\text { Iran, Iraq, Jordan, Saudi Arabia, Syria, } \\
\text { United Arab Emirates }\end{array}$ & $\begin{array}{l}\text { Armed Assault, Assassination, } \\
\text { Bombing/Explosion, Hostage Taking }\end{array}$ & $\begin{array}{l}\text { Government (General), Military, NGO, } \\
\text { Police, Private Citizens \& Property, } \\
\text { Religious Figures/Institutions }\end{array}$ \\
\hline al-Qaeda Serambi Mekkah & Religious & 2009 & - & Afghanistan, Indonesia, Iraq & $\begin{array}{l}\text { Armed Assault, Assassination, } \\
\text { Bombing/Explosion }\end{array}$ & Military \\
\hline al-Qaida in Iraq & Religious & 2004 & - & Iraq & $\begin{array}{l}\text { Armed Assault, Assassination, } \\
\text { Bombing/Explosion, } \\
\text { Facility/Infrastructure Attack, Hostage } \\
\text { Taking }\end{array}$ & $\begin{array}{l}\text { Airports \& Aircraft, Businesses, } \\
\text { Educational Institutes, Food or Water } \\
\text { Supply, Government (Diplomatic), } \\
\text { Government (General), Journalists \& } \\
\text { Media, Military, Police, Private Citizens } \\
\text { \& Property, Religious } \\
\text { Figures/Institutions, } \\
\text { Telecommunication, Terrorists/Non- } \\
\text { State Militia, Tourists, Transportation, } \\
\text { Utilities, Violent Political Party }\end{array}$ \\
\hline al-Qaida in Pakistan & Religious & 2002 & - & Pakistan & $\begin{array}{l}\text { Armed Assault, Assassination, } \\
\text { Bombing/Explosion }\end{array}$ & $\begin{array}{l}\text { Businesses, Government (General), } \\
\text { Military, Police, Private Citizens \& } \\
\text { Property, Religious Figures/Institutions, } \\
\text { Tourists, Violent Political Party }\end{array}$ \\
\hline al-Qaida in Saudi Arabia & Religious & 2003 & - & Saudi Arabia & Armed Assault, Bombing/Explosion & $\begin{array}{l}\text { Businesses, Government (General), } \\
\text { Private Citizens \& Property }\end{array}$ \\
\hline al-Qanoon/Lashkar-e-Omar & Religious & 2002 & 2002 & Pakistan & Bombing/Explosion & Government (Diplomatic) \\
\hline al-Qaqa Brigade & Nationalist & 2011 & - & Libya & $\begin{array}{l}\text { Armed Assault, Bombing/Explosion, } \\
\text { Hostage Taking }\end{array}$ & Military, Private Citizens \& Property \\
\hline al-Salafiya al-Jihadiya in Sinai & Religious & 2011 & - & Egypt, Libya & Armed Assault & Military \\
\hline al-Sawaiq Brigade & Nationalist & 2011 & - & Libya & Bombing/Explosion & Private Citizens \& Property \\
\hline al-Shabaab & Religious & 2004 & & $\begin{array}{l}\text { Australia, Egypt, Eritrea, Iran, Kenya, } \\
\text { Libya, Norway, Somalia, Sweden, } \\
\text { Uganda, United Kingdom, United States }\end{array}$ & $\begin{array}{l}\text { Armed Assault, Assassination, } \\
\text { Bombing/Explosion, } \\
\text { Facility/Infrastructure Attack, } \\
\text { Hijacking, Hostage Taking, Unarmed } \\
\text { Assault }\end{array}$ & $\begin{array}{l}\text { Airports \& Aircraft, Businesses, } \\
\text { Educational Institutes, Food or Water } \\
\text { Supply, Government (Diplomatic), } \\
\text { Government (General), Journalists \& } \\
\text { Media, Maritime, Military, NGO, Police, } \\
\text { Private Citizens \& Property, Religious } \\
\text { Figures/Institutions, } \\
\text { Telecommunication, Terrorists/Non- } \\
\text { State Militia, Tourists, Transportation, } \\
\text { Utilities }\end{array}$ \\
\hline al-Sham Legion & Religious & 2015 & - & Syria & Bombing/Explosion & Airports \& Aircraft, Businesses, Military \\
\hline al-Shuda Brigade & Religious & 2003 & - & India & $\begin{array}{l}\text { Armed Assault, Bombing/Explosion, } \\
\text { Hijacking }\end{array}$ & $\begin{array}{l}\text { Military, Police, Private Citizens \& } \\
\text { Property }\end{array}$ \\
\hline
\end{tabular}




\begin{tabular}{|c|c|c|c|c|c|c|}
\hline VEO Name & Type & Emerged & Ended & Countries & Type of Attack & Target Type \\
\hline al-Tawhid - Germany & Religious & 2001 & - & Germany & Armed Assault, Bombing/Explosion & $\begin{array}{l}\text { Businesses, Educational Institutes, } \\
\text { Government (General), Police, Private } \\
\text { Citizens \& Property, } \\
\text { Telecommunication, Transportation, } \\
\text { Utilities }\end{array}$ \\
\hline al-Tawhid Brigade & Nationalist & 2012 & - & Syria & Armed Assault, Bombing/Explosion & Government (General), Military \\
\hline al-Tawhid wal Jihad & Religious & 2002 & - & Egypt & Bombing/Explosion & $\begin{array}{l}\text { Businesses, Government (General), } \\
\text { Police, Private Citizens \& Property }\end{array}$ \\
\hline al-Ummah & Religious & 1993 & - & India & Assassination, Bombing/Explosion & $\begin{array}{l}\text { Private Citizens \& Property, Violent } \\
\text { Political Party }\end{array}$ \\
\hline $\begin{array}{l}\text { al-Zintan Revolutionaries' Military } \\
\text { Council (ZMC) }\end{array}$ & Nationalist & 2011 & - & Libya & Armed Assault, Bombing/Explosion & Terrorists/Non-State Militia \\
\hline $\begin{array}{l}\text { Amr Bil Maroof Wa Nahi Anil Munkir } \\
\text { Pakistan }\end{array}$ & Religious & 2007 & - & Pakistan & Bombing/Explosion, Hostage Taking & $\begin{array}{l}\text { Businesses, Government (General), } \\
\text { Private Citizens \& Property, } \\
\text { Terrorists/Non-State Militia }\end{array}$ \\
\hline Anarchist Attack Teams & Left-Wing & 2000 & 2000 & Greece & Bombing/Explosion & Businesses \\
\hline Anarchist Collective & Left-Wing & 2005 & - & Greece & Armed Assault, Bombing/Explosion & Police \\
\hline Anarchist Faction & Left-Wing & 1999 & 1999 & Greece & $\begin{array}{l}\text { Bombing/Explosion, } \\
\text { Facility/Infrastructure Attack }\end{array}$ & Businesses, Government (Diplomatic) \\
\hline Anarchist Faction for Subversion & Left-Wing & 1999 & 2000 & Greece & Bombing/Explosion & Government (Diplomatic) \\
\hline Anarchist Liberation Brigade & Left-Wing & 1998 & 1998 & Greece & Bombing/Explosion & Businesses \\
\hline Anarchist Street Patrol & Left-Wing & 1998 & 1998 & Greece & Bombing/Explosion & Government (General) \\
\hline Anarchist Struggle & Left-Wing & 2000 & 2000 & Greece & Facility/Infrastructure Attack & Government (Diplomatic) \\
\hline Anarchists Attack Group (AAG) & Left-Wing & 2000 & 2000 & Greece & Bombing/Explosion & Businesses \\
\hline Anarkhiki Omadha 20 Louli & Left-Wing & 2000 & 2002 & Greece & Bombing/Explosion & Businesses \\
\hline $\begin{array}{l}\text { Ansar Ahl al-Sunnah wal Jamaa/Army } \\
\text { of the Followers of Sunni Islam }\end{array}$ & Religious & 2004 & - & Iraq & Hostage Taking & Government (General), Military \\
\hline $\begin{array}{l}\text { Ansar al-Aseer/Ansarul-Aseer/Ansar al- } \\
\text { Aseer }\end{array}$ & Religious & 2013 & - & Pakistan & Armed Assault & $\begin{array}{l}\text { Military, Police, Terrorists/Non-State } \\
\text { Militia }\end{array}$ \\
\hline Ansar al-Deen Front/Ansar al-Din Front & Religious & 2014 & - & Syria & Hostage Taking & Military \\
\hline Ansar al-Din - Iraq/Defenders of al Din & Religious & 2004 & 2004 & Iraq & Hostage Taking & Private Citizens \& Property \\
\hline $\begin{array}{l}\text { Ansar al-Din al-Salafiya-Mali/Salafist } \\
\text { Warriors of Religion/Ansar al } \\
\text { Din/Ansar al-Dine/Ansar Uddin/Ansar } \\
\text { Eddin/Defenders of the Faith }\end{array}$ & Religious & 2011 & - & Mali & Armed Assault, Hostage Taking & Government (General), Military \\
\hline
\end{tabular}




\begin{tabular}{|c|c|c|c|c|c|c|}
\hline VEO Name & Type & Emerged & Ended & Countries & Type of Attack & $\begin{array}{c}\text { Target Type } \\
\end{array}$ \\
\hline Ansar al-Dine - Mali & Religious & 2012 & - & Mali, Mauritania & $\begin{array}{l}\text { Armed Assault, Bombing/Explosion, } \\
\text { Facility/Infrastructure Attack, Hostage } \\
\text { Taking, Unarmed Assault }\end{array}$ & $\begin{array}{l}\text { Businesses, Government (Diplomatic), } \\
\text { Government (General), Military, NGO, } \\
\text { Police, Private Citizens \& Property, } \\
\text { Religious Figures/Institutions, } \\
\text { Terrorists/Non-State Militia }\end{array}$ \\
\hline Ansar al-Fath & Religious & 2005 & 2005 & France & Bombing/Explosion & Military \\
\hline $\begin{array}{l}\text { Ansar Al-Furqan/Ansar Al Furqan Al } \\
\text { Mujahideen fi Iran }\end{array}$ & Nationalist & 2013 & - & Afghanistan, Iran, Pakistan & Bombing/Explosion & Unknown \\
\hline Ansar al-Islam & Religious & 2001 & - & Iraq & $\begin{array}{l}\text { Armed Assault, Assassination, } \\
\text { Bombing/Explosion, Hostage Taking }\end{array}$ & $\begin{array}{l}\text { Government (Diplomatic), Government } \\
\text { (General), Journalists \& Media, Military, } \\
\text { Police, Private Citizens \& Property, } \\
\text { Telecommunication, Terrorists/Non- } \\
\text { State Militia, Utilities, Violent Political } \\
\text { Party }\end{array}$ \\
\hline $\begin{array}{l}\text { Ansar al-Islam in the Muslim Sahara, } \\
\text { Land of the Veiled Ones (AIMS) }\end{array}$ & Religious & 2007 & - & Algeria, Morocco, Spain & Bombing/Explosion & Military \\
\hline Ansar al-Jihad & Religious & 2004 & 2004 & Iraq & Hostage Taking & Private Citizens \& Property \\
\hline $\begin{array}{l}\text { Ansar al-Khilafah Philippines/Islamic } \\
\text { State Philippines }\end{array}$ & Religious & 2014 & - & Indonesia, Malaysia, Philippines & Armed Assault, Bombing/Explosion & Businesses, Military, Police \\
\hline Ansar Allah & Religious & 1994 & 1994 & Lebanon, Panama & Bombing/Explosion & Airports \& Aircraft \\
\hline Ansar Allah al-Awfiyaa & Religious & 2014 & - & Iran, Iraq & Armed Assault & Terrorists/Non-State Militia \\
\hline Ansar al-Mahdi-Iraq & Religious & 2008 & - & Iraq & Armed Assault & Military \\
\hline Ansar al-Mahdi-Morocco & Religious & 2006 & 2006 & Morocco & Bombing/Explosion & Unknown \\
\hline Ansar al-Mujahideen & Religious & 2013 & - & Pakistan & Assassination, Bombing/Explosion & $\begin{array}{l}\text { Government (General), Military, Police, } \\
\text { Private Citizens \& Property }\end{array}$ \\
\hline Ansar al-Sharia & Religious & 2012 & & Egypt, Libya, Morocco, Yemen & $\begin{array}{l}\text { Armed Assault, Assassination, } \\
\text { Bombing/Explosion, Hostage Taking }\end{array}$ & $\begin{array}{l}\text { Airports \& Aircraft, Businesses, } \\
\text { Government (Diplomatic), Government } \\
\text { (General), Journalists \& Media, } \\
\text { Maritime, Military, Police, Private } \\
\text { Citizens \& Property, Religious } \\
\text { Figures/Institutions, Terrorists/Non- } \\
\text { State Militia, Utilities }\end{array}$ \\
\hline Ansar al-Sharia in Mauritania & Religious & 2013 & - & Mauritania & Armed Assault & $\begin{array}{l}\text { Government (General), Private Citizens } \\
\text { \& Property }\end{array}$ \\
\hline Ansar al-Sharia in Tunisia & Religious & 2011 & - & Tunisia & $\begin{array}{l}\text { Armed Assault, Assassination, } \\
\text { Bombing/Explosion, Hostage Taking }\end{array}$ & $\begin{array}{l}\text { Businesses, Government (Diplomatic), } \\
\text { Military, Police, Private Citizens \& } \\
\text { Property, Religious Figures/Institutions }\end{array}$ \\
\hline Ansar al-Shariah in the Central Regions & Religious & 2014 & - & Yemen & Unknown & Police, Private Citizens \& Property \\
\hline
\end{tabular}




\begin{tabular}{|c|c|c|c|c|c|c|}
\hline $\begin{array}{l}\text { VEO Name } \\
\end{array}$ & Type & Emerged & Ended & Countries & Type of Attack & Target Type \\
\hline $\begin{array}{l}\text { Ansar al-Sunna/Ansar al-Sunnah } \\
\text { Army/Defender's of the Prophet's } \\
\text { Traditions/Ansar al-Islam/Ansar Al- } \\
\text { Sunna Army/Devotees of } \\
\text { Islam/Followers of Islam in } \\
\text { Kurdistan/Helpers of Islam/Jaish Ansar } \\
\text { Al-Sunna/Jund Al-Islam/Kurdish } \\
\text { Taliban/Kurdistan Supporters of Isl }\end{array}$ & Religious & 2003 & - & Afghanistan, Iraq, Norway & $\begin{array}{l}\text { Armed Assault, Assassination, } \\
\text { Bombing/Explosion, Hostage Taking }\end{array}$ & $\begin{array}{l}\text { Businesses, Government (General), } \\
\text { Military, Police, Private Citizens \& } \\
\text { Property, Terrorists/Non-State Militia }\end{array}$ \\
\hline Ansar al-Sunnah & Religious & 2013 & - & Mali & Armed Assault, Bombing/Explosion & Private Citizens \& Property \\
\hline Ansar al-Sunnah - North Caucasus & Nationalist & 2013 & - & Russia & Bombing/Explosion & Private Citizens \& Property \\
\hline Ansar al-Sunnah Army & Religious & 2003 & - & Iraq & $\begin{array}{l}\text { Assassination, Bombing/Explosion, } \\
\text { Hostage Taking }\end{array}$ & $\begin{array}{l}\text { Businesses, Government (General), } \\
\text { Military, Police, Private Citizens \& } \\
\text { Property }\end{array}$ \\
\hline Ansar al-Tawhid wal Sunna & Religious & 2006 & 2006 & Iraq & Hostage Taking & Businesses \\
\hline Ansar Jerusalem/Ansar Bayt al-Maqdis & Religious & 2012 & - & Egypt, Palestinian State & $\begin{array}{l}\text { Armed Assault, Assassination, } \\
\text { Bombing/Explosion, Hijacking, Hostage } \\
\text { Taking }\end{array}$ & $\begin{array}{l}\text { Businesses, Government (General), } \\
\text { Military, Police, Private Citizens \& } \\
\text { Property, Tourists, Transportation, } \\
\text { Utilities }\end{array}$ \\
\hline Ansar Sarallah & Religious & 1999 & 1999 & Iran & Armed Assault & Government (General) \\
\hline Ansar ul-Islam & Religious & 2004 & - & Pakistan & Armed Assault, Assassination & $\begin{array}{l}\text { Educational Institutes, Private Citizens } \\
\text { \& Property }\end{array}$ \\
\hline Ansar Wa Mohajir & Religious & 2004 & - & Pakistan & Bombing/Explosion & $\begin{array}{l}\text { Government (General), Private Citizens } \\
\text { \& Property }\end{array}$ \\
\hline Ansar-e Hezbollah & Religious & 1995 & - & Iran & $\begin{array}{l}\text { Armed Assault, Assassination, } \\
\text { Bombing/Explosion, Hostage Taking }\end{array}$ & $\begin{array}{l}\text { Educational Institutes, Government } \\
\text { (General), Journalists \& Media, Private } \\
\text { Citizens \& Property }\end{array}$ \\
\hline Ansarour Islam - Burkina Faso & Nationalist & 2016 & - & Burkina Faso, Mali & Armed Assault & $\begin{array}{l}\text { Government (General), Military, } \\
\text { Religious Figures/Institutions }\end{array}$ \\
\hline Ansarullah Bangla Team (ABT) & Religious & 2007 & - & Bangladesh, Pakistan, Yemen & $\begin{array}{l}\text { Armed Assault, Assassination, Hostage } \\
\text { Taking }\end{array}$ & $\begin{array}{l}\text { Businesses, Educational Institutes, } \\
\text { Journalists \& Media, Private Citizens \& } \\
\text { Property, Religious Figures/Institutions, } \\
\text { Violent Political Party }\end{array}$ \\
\hline Ansar-ul-Mujahidin & Religious & 2013 & - & Pakistan & Armed Assault, Bombing/Explosion & Military, Private Citizens \& Property \\
\hline $\begin{array}{l}\text { Anti-Apostate Movement Alliance } \\
\text { (AGAP) }\end{array}$ & Religious & 2007 & - & Indonesia & Unarmed Assault & Religious Figures/Institutions \\
\hline
\end{tabular}




\begin{tabular}{|c|c|c|c|c|c|c|}
\hline VEO Name & Type & Emerged & Ended & Countries & Type of Attack & Target Type \\
\hline Anti-Balaka & Religious & 2013 & - & Central African Republic & $\begin{array}{l}\text { Armed Assault, Assassination, } \\
\text { Bombing/Explosion, Hostage Taking }\end{array}$ & $\begin{array}{l}\text { Government (Diplomatic), Government } \\
\text { (General), Military, NGO, Police, Private } \\
\text { Citizens \& Property, Religious } \\
\text { Figures/Institutions, Terrorists/Non- } \\
\text { State Militia, Transportation, Violent } \\
\text { Political Party }\end{array}$ \\
\hline Anti-Capitalist Action & Left-Wing & 1999 & 1999 & Greece & Bombing/Explosion & Businesses \\
\hline Anticapitalist Attack Nuclei & Left-Wing & 2001 & 2001 & Italy & Bombing/Explosion & Businesses \\
\hline Anti-Communist Command (KAK) & $\begin{array}{l}\text { Right- } \\
\text { Wing }\end{array}$ & 2000 & 2000 & Indonesia & Bombing/Explosion & NGO \\
\hline Anti-Establishment Nucleus & Left-Wing & 1995 & 1995 & Greece & Bombing/Explosion & Businesses \\
\hline Antifascistisk Aktion & Left-Wing & 1993 & 1993 & Sweden & Unknown & $\begin{array}{l}\text { Government (General), Private Citizens } \\
\text { \& Property }\end{array}$ \\
\hline Anti-Imperialist Cell (AIZ) & Left-Wing & 1994 & 1996 & Germany & Bombing/Explosion & Government (General) \\
\hline $\begin{array}{l}\text { Anti-Imperialist Group Liberty for } \\
\text { Mumia Abu Jamal }\end{array}$ & Left-Wing & 1995 & 1995 & Germany & Bombing/Explosion & Businesses \\
\hline $\begin{array}{l}\text { Anti-Imperialist Territorial Nuclei for } \\
\text { the Construction of the Fighting } \\
\text { Communist Party }\end{array}$ & Left-Wing & 1995 & 2003 & Italy & $\begin{array}{l}\text { Bombing/Explosion, } \\
\text { Facility/Infrastructure Attack }\end{array}$ & Government (General) \\
\hline Anti-Olympic Flame & Left-Wing & 2006 & 2006 & Greece & Bombing/Explosion & Telecommunication \\
\hline Anti-Power Struggle & Left-Wing & 1995 & 2001 & Greece & Bombing/Explosion & $\begin{array}{l}\text { Educational Institutes, Government } \\
\text { (General), NGO }\end{array}$ \\
\hline Anti-Racist Guerrilla Nuclei & Left-Wing & 1999 & 1999 & Italy & Bombing/Explosion & Educational Institutes \\
\hline Anti-State Defense & Left-Wing & 2006 & 2006 & Greece & Bombing/Explosion & Businesses \\
\hline Anti-State Justice & Left-Wing & 2006 & 2006 & Greece & Facility/Infrastructure Attack & $\begin{array}{l}\text { Government (Diplomatic), Government } \\
\text { (General), Private Citizens \& Property }\end{array}$ \\
\hline Anti-State Proletarian Nuclei & Left-Wing & 1999 & 1999 & Greece & Bombing/Explosion & Government (Diplomatic) \\
\hline Anti-Zionist Movement & $\begin{array}{l}\text { Right- } \\
\text { Wing }\end{array}$ & 1999 & 2000 & Italy & Bombing/Explosion & Businesses, Private Citizens \& Property \\
\hline Apo's Revenge Hawks & Nationalist & 1999 & 1999 & Turkey & Bombing/Explosion & $\begin{array}{l}\text { Police, Private Citizens \& Property, } \\
\text { Telecommunication }\end{array}$ \\
\hline Apo's Youth Revenge Brigades & Nationalist & 1999 & 1999 & Turkey & Bombing/Explosion & Government (General) \\
\hline Arab Movement of Azawad (MAA) & Nationalist & 2012 & - & Mali & $\begin{array}{l}\text { Armed Assault, Bombing/Explosion, } \\
\text { Facility/Infrastructure Attack, Hostage } \\
\text { Taking }\end{array}$ & $\begin{array}{l}\text { Businesses, Military, Police, Private } \\
\text { Citizens \& Property }\end{array}$ \\
\hline $\begin{array}{l}\text { Arab Struggle Movement for the } \\
\text { Liberation of Ahvaz }\end{array}$ & Nationalist & 2005 & - & Iran & Bombing/Explosion & Private Citizens \& Property \\
\hline
\end{tabular}




\begin{tabular}{|c|c|c|c|c|c|c|}
\hline VEO Name & Type & Emerged & Ended & $\begin{array}{c}\text { Countries } \\
\end{array}$ & Type of Attack & $\begin{array}{c}\text { Target Type } \\
\end{array}$ \\
\hline Arakan Rohingya National Organisation & Nationalist & 2001 & - & Bangladesh, Myanmar, Burma & Armed Assault & Military, Private Citizens \& Property \\
\hline $\begin{array}{l}\text { Arakan Rohingya Salvation Army } \\
\text { (ARSA) }\end{array}$ & Religious & 2016 & - & Myanmar, Burma & Armed Assault & Government (General), Police \\
\hline $\begin{array}{l}\text { Arauco-Malleco Coordinating Group } \\
\text { (CAM) }\end{array}$ & Nationalist & 1998 & - & Chile & Facility/Infrastructure Attack & Businesses, Government (General) \\
\hline Arbav Martyrs of Khuzestan & Nationalist & 2005 & 2005 & Iran & Bombing/Explosion & Private Citizens \& Property \\
\hline Armata Corsa & Nationalist & 1999 & 2001 & France & Bombing/Explosion & $\begin{array}{l}\text { Government (General), Private Citizens } \\
\text { \& Property }\end{array}$ \\
\hline Armata di Liberzione Naziunale (ALN) & Nationalist & 2002 & 2003 & France & Assassination, Bombing/Explosion & $\begin{array}{l}\text { Government (General), Police, } \\
\text { Terrorists/Non-State Militia, Utilities }\end{array}$ \\
\hline $\begin{array}{l}\text { Armed Forces for the Federal Republic } \\
\text { (FARF) }\end{array}$ & Nationalist & 1994 & 1998 & Chad & Armed Assault & Military \\
\hline $\begin{array}{l}\text { Armed Forces of the Chechen Republic } \\
\text { of Ichkeria }\end{array}$ & Nationalist & 2001 & - & Russia & Armed Assault, Bombing/Explosion & $\begin{array}{l}\text { Businesses, Government (General), } \\
\text { Military, Police }\end{array}$ \\
\hline $\begin{array}{l}\text { Armed Forces Revolutionary Council } \\
\text { (AFRC) }\end{array}$ & Left-Wing & 1999 & 1999 & Sierra Leone & Armed Assault, Hostage Taking & $\begin{array}{l}\text { Private Citizens \& Property, Religious } \\
\text { Figures/Institutions }\end{array}$ \\
\hline $\begin{array}{l}\text { Armed Islamic Group (AIG) of al-Qaeda } \\
\text { in Fallujah and Ramadi }\end{array}$ & Religious & 2003 & - & Iraq & Armed Assault & Military, Police \\
\hline Armed Islamic Group (GIA) & Religious & 1992 & 2006 & Algeria & $\begin{array}{l}\text { Armed Assault, Assassination, } \\
\text { Bombing/Explosion, } \\
\text { Facility/Infrastructure Attack, } \\
\text { Hijacking, Hostage Taking }\end{array}$ & $\begin{array}{l}\text { Airports \& Aircraft, Businesses, } \\
\text { Educational Institutes, Government } \\
\text { (Diplomatic), Journalists \& Media, } \\
\text { Maritime, Military, Police, Private } \\
\text { Citizens \& Property, Religious } \\
\text { Figures/Institutions, Terrorists/Non- } \\
\text { State Militia, Transportation, Utilities }\end{array}$ \\
\hline Armed Islamic Movement (MIA) & Religious & 1992 & 1992 & Algeria & Armed Assault & Police \\
\hline $\begin{array}{l}\text { Armed Revolutionary Action - Greece } \\
\text { (ENEDRA) }\end{array}$ & Left-Wing & 2008 & - & Greece & Bombing/Explosion & Government (General) \\
\hline Armed Revolutionary Left & Left-Wing & 2004 & 2004 & Ecuador & Assassination, Bombing/Explosion & Government (General) \\
\hline Armenian Resistance Group & Nationalist & 1995 & 1995 & Armenia, Belgium, Netherlands, Turkey & Bombing/Explosion & Government (General) \\
\hline Army for the Liberation of Rwanda & Nationalist & 1994 & 2002 & Congo, Republic of & $\begin{array}{l}\text { Armed Assault, Bombing/Explosion, } \\
\text { Hostage Taking, Unarmed Assault }\end{array}$ & $\begin{array}{l}\text { Government (General), Private Citizens } \\
\text { \& Property }\end{array}$ \\
\hline Army of Islam & Religious & 2007 & - & Egypt, Palestinian State, Syria & Bombing/Explosion, Hostage Taking & $\begin{array}{l}\text { Journalists \& Media, Private Citizens \& } \\
\text { Property }\end{array}$ \\
\hline Army of State Liberators & Nationalist & 2008 & - & Israel & Bombing/Explosion & Educational Institutes \\
\hline Army of the Corsican People & Nationalist & 2004 & 2004 & France & Bombing/Explosion & Government (General) \\
\hline
\end{tabular}




\begin{tabular}{|c|c|c|c|c|c|c|}
\hline VEO Name & Type & Emerged & Ended & Countries & Type of Attack & Target Type \\
\hline $\begin{array}{l}\text { Army of the Emigrants and } \\
\text { Helpers/Jaish al-Muhajireen wa al- } \\
\text { Ansar }\end{array}$ & Religious & 2013 & - & Kuwait, Russia, Syria, Turkey & $\begin{array}{l}\text { Armed Assault, Assassination, } \\
\text { Bombing/Explosion }\end{array}$ & Government (General), Military \\
\hline Arsonists for Social Cohesion & Left-Wing & 1998 & 1998 & Greece & Facility/Infrastructure Attack & Businesses, Government (Diplomatic) \\
\hline Arunachal Dragon Force (EILF) & Nationalist & 1996 & - & India & Unknown & Unknown \\
\hline Asa'ib Ahl al-Haq (AAH) & Religious & 2004 & - & Iraq, Syria & $\begin{array}{l}\text { Armed Assault, Bombing/Explosion, } \\
\text { Facility/Infrastructure Attack, Hostage } \\
\text { Taking }\end{array}$ & $\begin{array}{l}\text { Businesses, Government (Diplomatic), } \\
\text { Military, Private Citizens \& Property, } \\
\text { Religious Figures/Institutions }\end{array}$ \\
\hline Athens and Thessaloniki Arsonist Nuclei & Left-Wing & 2007 & 2007 & Greece & Bombing/Explosion & $\begin{array}{l}\text { Businesses, Educational Institutes, } \\
\text { Government (General) }\end{array}$ \\
\hline $\begin{array}{l}\text { Atika/Movement of Democratic Forces } \\
\text { of Casamance (MFDC) }\end{array}$ & Nationalist & 2001 & - & Senegal & Unknown & Military \\
\hline $\begin{array}{l}\text { Attack Teams for the Dissolution of the } \\
\text { Nation }\end{array}$ & Left-Wing & 2009 & 2009 & Greece & $\begin{array}{l}\text { Bombing/Explosion, } \\
\text { Facility/Infrastructure Attack }\end{array}$ & Government (General), NGO \\
\hline Autodefensas Campensinas del Cassnare & Left-Wing & 2004 & - & Colombia & Armed Assault & Government (General) \\
\hline $\begin{array}{l}\text { Autonomous and Destructive Forces } \\
\text { Leon Czolgosz (ADFLC) }\end{array}$ & Left-Wing & 2006 & - & Chile & Bombing/Explosion & $\begin{array}{l}\text { Government (Diplomatic), Government } \\
\text { (General) }\end{array}$ \\
\hline $\begin{array}{l}\text { Autonomous Cells of Rebel Action } \\
\text { (ACRA) }\end{array}$ & Left-Wing & 1998 & 1998 & Greece & Bombing/Explosion & Businesses \\
\hline $\begin{array}{l}\text { Autonomous Combat Terrorist } \\
\text { Organization }\end{array}$ & $\begin{array}{l}\text { Right- } \\
\text { Wing }\end{array}$ & 2009 & - & Russia & Bombing/Explosion & $\begin{array}{l}\text { Businesses, Police, Private Citizens \& } \\
\text { Property }\end{array}$ \\
\hline Autonomous Decorators & Left-Wing & 1999 & 2000 & Germany & Armed Assault, Bombing/Explosion & Businesses, Government (General) \\
\hline Azerbaijani Jamaat & Religious & 2008 & - & Azerbaijan, Russia, Syria & Armed Assault, Bombing/Explosion & Military \\
\hline Azeri Group & Nationalist & 2007 & - & Azerbaijan & Unknown & $\begin{array}{l}\text { Government (General), Religious } \\
\text { Figures/Institutions }\end{array}$ \\
\hline Baby Liberation Army & $\begin{array}{l}\text { Right- } \\
\text { Wing }\end{array}$ & 2000 & 2000 & Canada & Armed Assault & Abortion Related \\
\hline Badr Corps/Badr Forces & Nationalist & 2001 & 2001 & Iran, Iraq, Syria & $\begin{array}{l}\text { Armed Assault, Assassination, } \\
\text { Bombing/Explosion }\end{array}$ & $\begin{array}{l}\text { Government (General), Private Citizens } \\
\text { \& Property }\end{array}$ \\
\hline Baggaras Militia & Religious & 2003 & - & Sudan & Armed Assault, Assassination & Terrorists/Non-State Militia \\
\hline Bagramyan Battalion & Nationalist & 1998 & 1998 & Georgia & Unknown & $\begin{array}{l}\text { Military, Private Citizens \& Property, } \\
\text { Utilities }\end{array}$ \\
\hline Baha' Alyan Groups & Nationalist & 2016 & - & Israel, Palestinian State & Unknown & Police \\
\hline Bakassi Strike Force (BSF) & Nationalist & 2016 & - & Cameroon, Nigeria & Armed Assault & Government (General) \\
\hline Bakata Katanga & Nationalist & 2011 & - & Congo, Democratic Republic & Armed Assault & Private Citizens \& Property \\
\hline
\end{tabular}




\begin{tabular}{|c|c|c|c|c|c|c|}
\hline VEO Name & Type & Emerged & Ended & Countries & Type of Attack & Target Type \\
\hline $\begin{array}{l}\text { Baloch Liberation Army (BLA)/Baloch } \\
\text { Waja Liberation Army (BWLA) }\end{array}$ & Nationalist & 2000 & - & Pakistan & $\begin{array}{l}\text { Armed Assault, Assassination, } \\
\text { Bombing/Explosion, } \\
\text { Facility/Infrastructure Attack, Hostage } \\
\text { Taking }\end{array}$ & $\begin{array}{l}\text { Businesses, Maritime, Military, Police, } \\
\text { Private Citizens \& Property, } \\
\text { Transportation, Utilities }\end{array}$ \\
\hline Baloch Liberation Tigers (BLT) & Nationalist & 2011 & - & Pakistan & Armed Assault, Bombing/Explosion & $\begin{array}{l}\text { Businesses, Government (General), } \\
\text { Police, Private Citizens \& Property }\end{array}$ \\
\hline Baloch Republican Army (BRA) & Nationalist & 2007 & - & Pakistan & $\begin{array}{l}\text { Armed Assault, Assassination, } \\
\text { Bombing/Explosion, } \\
\text { Facility/Infrastructure Attack, } \\
\text { Hijacking, Hostage Taking }\end{array}$ & $\begin{array}{l}\text { Airports \& Aircraft, Businesses, } \\
\text { Educational Institutes, Food or Water } \\
\text { Supply, Government (General), Military, } \\
\text { Police, Private Citizens \& Property, } \\
\text { Transportation, Utilities }\end{array}$ \\
\hline Baloch Republican Guards (BRG) & Nationalist & 2014 & - & Pakistan & Bombing/Explosion & $\begin{array}{l}\text { Businesses, Military, Private Citizens \& } \\
\text { Property, Transportation }\end{array}$ \\
\hline Balochistan Bunyad Parast Army & Nationalist & 2012 & - & Pakistan & Armed Assault, Assassination & Government (General) \\
\hline $\begin{array}{l}\text { Balochistan Liberation United Front } \\
\text { (BLUF) }\end{array}$ & Nationalist & 2009 & - & Pakistan & $\begin{array}{l}\text { Armed Assault, Assassination, } \\
\text { Bombing/Explosion }\end{array}$ & $\begin{array}{l}\text { Businesses, Government (General), } \\
\text { Military, Police, Private Citizens \& } \\
\text { Property, Religious Figures/Institutions, } \\
\text { Utilities, Violent Political Party }\end{array}$ \\
\hline $\begin{array}{l}\text { Balochistan Musalla Difa Tanzeem } \\
\text { (BMDT) }\end{array}$ & Nationalist & 2010 & - & Pakistan & Armed Assault, Bombing/Explosion & $\begin{array}{l}\text { Government (General), Private Citizens } \\
\text { \& Property }\end{array}$ \\
\hline Balochistan National Army & Nationalist & 1999 & 2004 & Iran, Pakistan & Bombing/Explosion & Businesses, Military, Transportation \\
\hline $\begin{array}{l}\text { Balochistan Republican Party Azad } \\
\text { (BRPA) }\end{array}$ & Nationalist & 2012 & - & Pakistan & Armed Assault & Police, Utilities \\
\hline $\begin{array}{l}\text { Balochistan Waja Liberation Army } \\
\text { (BWA) }\end{array}$ & Nationalist & 2012 & - & Pakistan & $\begin{array}{l}\text { Armed Assault, Bombing/Explosion, } \\
\text { Hostage Taking }\end{array}$ & Businesses, Police \\
\hline $\begin{array}{l}\text { Banda Dinamitera Efrain Plaza Olmedo } \\
\text { (BDEP) }\end{array}$ & Left-Wing & 2009 & - & Chile & Bombing/Explosion & Businesses \\
\hline Bangladesh National Socialist Party & Left-Wing & 1992 & 1992 & Bangladesh & Armed Assault & Private Citizens \& Property \\
\hline Bangsamoro Independence Movement & Nationalist & 2012 & - & Bangladesh & Bombing/Explosion & Government (General) \\
\hline $\begin{array}{l}\text { Bangsamoro Islamic Freedom Fighters } \\
\text { (BIFF)/Bangsamoro Islamic Freedom } \\
\text { Movement (BIFM) }\end{array}$ & Religious & 2008 & - & Philippines & $\begin{array}{l}\text { Armed Assault, Assassination, } \\
\text { Bombing/Explosion, } \\
\text { Facility/Infrastructure Attack, Hostage } \\
\text { Taking }\end{array}$ & $\begin{array}{l}\text { Military, Private Citizens \& Property, } \\
\text { Religious Figures/Institutions }\end{array}$ \\
\hline Bangsamoro Justice Movement (BJM) & Nationalist & 2014 & - & Philippines & Unknown & Unknown \\
\hline Banner of Islam & Nationalist & 2006 & - & Iraq & Hostage Taking & Government (Diplomatic) \\
\hline Barq al-Nasser Brigade & Religious & 2014 & - & Libya & Hostage Taking & Government (General) \\
\hline
\end{tabular}




\begin{tabular}{|c|c|c|c|c|c|c|}
\hline VEO Name & Type & Emerged & Ended & Countries & Type of Attack & Target Type \\
\hline Barqa Province of the Islamic State & Religious & 2014 & - & Libya & $\begin{array}{l}\text { Armed Assault, Assassination, } \\
\text { Bombing/Explosion, } \\
\text { Facility/Infrastructure Attack, } \\
\text { Hijacking, Hostage Taking }\end{array}$ & $\begin{array}{l}\text { Businesses, Educational Institutes, } \\
\text { Government (General), Journalists \& } \\
\text { Media, Military, Police, Religious } \\
\text { Figures/Institutions, Terrorists/Non- } \\
\text { State Militia, Utilities }\end{array}$ \\
\hline Barqa Youth Movement & Religious & 2013 & - & Libya & Facility/Infrastructure Attack & Utilities \\
\hline Bat Ayin Group & Religious & 2011 & - & Israel & Armed Assault & Private Citizens \& Property \\
\hline $\begin{array}{l}\text { Battalion of the Look-out for } \\
\text { Iraq/Brigade of Monitoring for Iraq }\end{array}$ & Nationalist & 2005 & - & Iraq & Hostage Taking & NGO \\
\hline Bavarian Liberation Army (BLA) & $\begin{array}{l}\text { Right- } \\
\text { Wing }\end{array}$ & 1995 & 1998 & Austria, Germany, Romania & Bombing/Explosion & $\begin{array}{l}\text { Government (General), Private Citizens } \\
\text { \& Property }\end{array}$ \\
\hline Bay'at & Religious & 2003 & - & Tajikistan & Bombing/Explosion & $\begin{array}{l}\text { Businesses, Religious } \\
\text { Figures/Institutions }\end{array}$ \\
\hline Belarusian Liberation Army (NLA) & Nationalist & 1997 & - & Austria, Belarus & Bombing/Explosion & $\begin{array}{l}\text { Businesses, Government (General), } \\
\text { Private Citizens \& Property, } \\
\text { Transportation }\end{array}$ \\
\hline Bengali Tiger Force & Nationalist & 1999 & 1999 & India & Armed Assault & Private Citizens \& Property \\
\hline Bhutan Tiger Force (BTF) & Left-Wing & 2003 & - & Bhutan & Armed Assault, Bombing/Explosion & Government (General) \\
\hline Biat al-Imam & Religious & 1992 & 1994 & Jordan & Bombing/Explosion & Private Citizens \& Property \\
\hline Bihar People's Party & Religious & 1994 & 1994 & India & Assassination & Government (General) \\
\hline Birsa Commando Force & Nationalist & 1996 & 2004 & India & Unknown & Private Citizens \& Property \\
\hline $\begin{array}{l}\text { Black and Red Anarchist and Anti- } \\
\text { Authoritarians Initiative }\end{array}$ & Left-Wing & 2009 & 2009 & Greece & Facility/Infrastructure Attack & Government (General) \\
\hline Black and Red Brigades & Left-Wing & 2004 & - & Greece & Unknown & Government (General) \\
\hline Black Banner Brigade & Left-Wing & 2004 & - & Iraq & Hostage Taking & Businesses \\
\hline Black Bloc Egypt & Left-Wing & 2012 & - & Egypt & Bombing/Explosion & Police \\
\hline Black Hawks Anti-Wahhabists & Nationalist & 2011 & - & Russia & $\begin{array}{l}\text { Armed Assault, Bombing/Explosion, } \\
\text { Facility/Infrastructure Attack }\end{array}$ & Terrorists/Non-State Militia \\
\hline Black Malian Group & $\begin{array}{l}\text { Right- } \\
\text { Wing }\end{array}$ & 1992 & 1992 & Mali & Armed Assault & Private Citizens \& Property \\
\hline Black Star & Left-Wing & 1999 & 2002 & Greece & $\begin{array}{l}\text { Bombing/Explosion, } \\
\text { Facility/Infrastructure Attack }\end{array}$ & $\begin{array}{l}\text { Businesses, Government (Diplomatic), } \\
\text { Government (General), Police }\end{array}$ \\
\hline Black Widows & Nationalist & 2003 & - & India & $\begin{array}{l}\text { Armed Assault, Bombing/Explosion, } \\
\text { Facility/Infrastructure Attack }\end{array}$ & $\begin{array}{l}\text { Military, Police, Private Citizens \& } \\
\text { Property, Terrorists/Non-State Militia, } \\
\text { Transportation }\end{array}$ \\
\hline
\end{tabular}




\begin{tabular}{|c|c|c|c|c|c|c|}
\hline $\begin{array}{l}\text { VEO Name } \\
\end{array}$ & Type & Emerged & Ended & Countries & $\begin{array}{c}\text { Type of Attack } \\
\end{array}$ & $\begin{array}{c}\text { Target Type } \\
\end{array}$ \\
\hline Bodo Liberation Tigers (BLT) & Nationalist & 1996 & 2003 & Bhutan, India, Pakistan & $\begin{array}{l}\text { Armed Assault, Bombing/Explosion, } \\
\text { Facility/Infrastructure Attack }\end{array}$ & $\begin{array}{l}\text { Government (General), NGO, Police, } \\
\text { Private Citizens \& Property, } \\
\text { Transportation }\end{array}$ \\
\hline Bodu Bala Sena (BBS) & Religious & 2012 & - & Sri Lanka & Facility/Infrastructure Attack & Private Citizens \& Property \\
\hline Boer Republikeinse Leer & Nationalist & 1992 & 1992 & South Africa & Bombing/Explosion & Private Citizens \& Property \\
\hline Boere Aanvals Troepe (BAT) & $\begin{array}{l}\text { Right- } \\
\text { Wing }\end{array}$ & 1996 & 1997 & South Africa & Bombing/Explosion & $\begin{array}{l}\text { Businesses, Government (General), } \\
\text { Religious Figures/Institutions }\end{array}$ \\
\hline Boerestaat Party & $\begin{array}{l}\text { Right- } \\
\text { Wing }\end{array}$ & 1999 & 1999 & South Africa & Bombing/Explosion & Government (General), NGO \\
\hline Boko Haram & Religious & 2012 & - & Cameroon, Chad, Niger, Nigeria & $\begin{array}{l}\text { Armed Assault, Assassination, } \\
\text { Bombing/Explosion, } \\
\text { Facility/Infrastructure Attack, } \\
\text { Hijacking, Hostage Taking }\end{array}$ & $\begin{array}{l}\text { Airports \& Aircraft, Businesses, } \\
\text { Educational Institutes, Government } \\
\text { (Diplomatic), Government (General), } \\
\text { Military, NGO, Police, Private Citizens \& } \\
\text { Property, Religious Figures/Institutions, } \\
\text { Telecommunication, Terrorists/Non- } \\
\text { State Militia, Tourists, Transportation, } \\
\text { Violent Political Party }\end{array}$ \\
\hline Bolivarian Guerilla Movement (MGB) & Left-Wing & 2003 & 2003 & Venezuela & Bombing/Explosion & Government (General) \\
\hline Bolivarian Liberation Forces (FBL) & Left-Wing & 1992 & - & Venezuela & $\begin{array}{l}\text { Assassination, Bombing/Explosion, } \\
\text { Hostage Taking }\end{array}$ & Government (General) \\
\hline Bologna 12 & Left-Wing & 2007 & - & Italy & Unknown & Government (General) \\
\hline Borok National Council of Tripura & Nationalist & 2000 & 2000 & Bangladesh, India & $\begin{array}{l}\text { Armed Assault, Assassination, Hostage } \\
\text { Taking }\end{array}$ & Violent Political Party \\
\hline Bougainville Freedom Fighters (BFF) & Nationalist & 2006 & - & Australia, Papua New Guinea & Armed Assault & Military \\
\hline Bozize Militia & Nationalist & 2001 & 2003 & Central African Republic, Chad & Armed Assault & Military, Violent Political Party \\
\hline Brigade 313 & Religious & 2003 & - & Pakistan, Syria & Bombing/Explosion & $\begin{array}{l}\text { Private Citizens \& Property, } \\
\text { Terrorists/Non-State Militia }\end{array}$ \\
\hline Brigade of Aisha, Mother of Believers & Religious & 2013 & - & Lebanon & Bombing/Explosion & Violent Political Party \\
\hline Brigade of al-Mukhtar-al-Thaqafi & Religious & 2012 & - & Lebanon & Hostage Taking & Terrorists/Non-State Militia \\
\hline Brigade of Ansar al-Tawhid wa-Sunna & Nationalist & 2006 & - & Iraq & Hostage Taking & Private Citizens \& Property \\
\hline Brigade of Islam & Nationalist & 2011 & - & Syria & Bombing/Explosion & Government (General) \\
\hline $\begin{array}{l}\text { Brigade of the Gallant Companion of the } \\
\text { Prophet Mohammed bin Muslima }\end{array}$ & Religious & 2011 & 2011 & Palestinian State & Armed Assault, Hostage Taking & Private Citizens \& Property \\
\hline $\begin{array}{l}\text { Brigade of the Martyr Abu Anas al- } \\
\text { Shami }\end{array}$ & Religious & 2004 & - & Saudi Arabia & Armed Assault & Government (Diplomatic) \\
\hline Brigade of the Two Holy Mosques & Religious & 2000 & - & Saudi Arabia & Bombing/Explosion & Military \\
\hline Brigades for the Defense of Holy Shrines & Religious & 2004 & - & Iraq & Hostage Taking & Military \\
\hline
\end{tabular}




\begin{tabular}{|c|c|c|c|c|c|c|}
\hline VEO Name & Type & Emerged & Ended & Countries & Type of Attack & Target Type \\
\hline Brigades of Imam al-Hassan al-Basri & Religious & 2005 & 2005 & Iraq & $\begin{array}{l}\text { Armed Assault, Assassination, } \\
\text { Bombing/Explosion, Hostage Taking }\end{array}$ & $\begin{array}{l}\text { Government (General), Journalists \& } \\
\text { Media, Police }\end{array}$ \\
\hline $\begin{array}{l}\text { Brigades of Martyr Atta al-Qaeda } \\
\text { Organization }\end{array}$ & Religious & 2004 & - & El Salvador & Unknown & Government (General) \\
\hline Brigades of Martyr Ahmed Yassin & Nationalist & 2004 & 2004 & Iraq & $\begin{array}{l}\text { Armed Assault, Assassination, } \\
\text { Bombing/Explosion }\end{array}$ & Government (General), Military \\
\hline Brigades of Osama Bin Laden & Religious & 2012 & - & Palestinian State & Armed Assault & Unknown \\
\hline Brigades of Popular Resistance & Religious & 2014 & - & Bahrain, Iran & Armed Assault, Bombing/Explosion & $\begin{array}{l}\text { Government (General), Religious } \\
\text { Figures/Institutions }\end{array}$ \\
\hline $\begin{array}{l}\text { Brigades of the Martyr Mohieldain al- } \\
\text { Naser }\end{array}$ & Nationalist & 2013 & - & Iran & Bombing/Explosion & Utilities \\
\hline Brigades of the Victorious Lion of God & Religious & 2004 & - & Iraq & Bombing/Explosion, Hostage Taking & Government (General), Military \\
\hline Bru National Liberation Front (BNLF) & Nationalist & 1996 & - & India & Hostage Taking & $\begin{array}{l}\text { Police, Private Citizens \& Property, } \\
\text { Religious Figures/Institutions }\end{array}$ \\
\hline Bunda Dia Kongo (BDK) & Nationalist & 2007 & - & Congo, Democratic Republic & Armed Assault & Private Citizens \& Property \\
\hline Burkha Brigade & Religious & 2012 & - & Russia & Armed Assault, Bombing/Explosion & Military \\
\hline Burning Path & Left-Wing & 2002 & - & Greece & Bombing/Explosion & Businesses, Government (General) \\
\hline Byeloye Bratsva & Religious & 1993 & 1994 & Russia, Ukraine & Bombing/Explosion & Utilities \\
\hline C14 aka Sich & Nationalist & 2010 & - & Ukraine & Armed Assault, Bombing/Explosion & $\begin{array}{l}\text { Government (General), Private Citizens } \\
\text { \& Property, Terrorists/Non-State Militia }\end{array}$ \\
\hline Cambodian Freedom Fighters (CFF) & Left-Wing & 1998 & 2001 & Cambodia, United States & Armed Assault, Bombing/Explosion & Government (General), Military, Police \\
\hline Cambodian People's Party & Left-Wing & 1997 & 1997 & Cambodia & Armed Assault, Bombing/Explosion & $\begin{array}{l}\text { Government (General), Private Citizens } \\
\text { \& Property, Terrorists/Non-State Militia }\end{array}$ \\
\hline Cannibal Army & Nationalist & 2003 & 2003 & Haiti & Facility/Infrastructure Attack & Government (General), Police \\
\hline Caprivi Liberation Army & Nationalist & 1998 & 1999 & Botswana, Namibia, Zambia & Armed Assault & Government (General) \\
\hline Carapaica Revolutionary Movement & Left-Wing & 2002 & - & Venezuela & Armed Assault & Police \\
\hline CasaPound (CP) & $\begin{array}{l}\text { Right- } \\
\text { Wing }\end{array}$ & 2003 & - & Italy & Armed Assault & $\begin{array}{l}\text { Government (General), Private Citizens } \\
\text { \& Property }\end{array}$ \\
\hline Caucasian Mujahadeen & Nationalist & 2009 & - & Russia & Bombing/Explosion & Transportation, Utilities \\
\hline Caucasus Emirate (CE) & Nationalist & 2005 & - & Belgium, Russia, Syria & $\begin{array}{l}\text { Armed Assault, Assassination, } \\
\text { Bombing/Explosion, } \\
\text { Facility/Infrastructure Attack, Hostage } \\
\text { Taking }\end{array}$ & $\begin{array}{l}\text { Airports \& Aircraft, Businesses, } \\
\text { Educational Institutes, Government } \\
\text { (General), Journalists \& Media, Police, } \\
\text { Private Citizens \& Property, Religious } \\
\text { Figures/Institutions, Transportation, } \\
\text { Utilities }\end{array}$ \\
\hline Caucasus Islamic Army & Religious & 2009 & - & Azerbaijan & Bombing/Explosion & Transportation, Utilities \\
\hline
\end{tabular}




\begin{tabular}{|c|c|c|c|c|c|c|}
\hline VEO Name & Type & Emerged & Ended & Countries & Type of Attack & Target Type \\
\hline Caucasus Mujahideen in Khorasan & Religious & 2011 & - & Afghanistan, Pakistan & Armed Assault & Military, Private Citizens \& Property \\
\hline$\overline{\mathrm{CCCCC}}$ & Left-Wing & 2002 & 2002 & Italy, Spain & Bombing/Explosion & $\begin{array}{l}\text { Airports \& Aircraft, Businesses, } \\
\text { Educational Institutes, Journalists \& } \\
\text { Media, Private Citizens \& Property }\end{array}$ \\
\hline Cell for Internationalism & Left-Wing & 1995 & 1995 & Austria & Facility/Infrastructure Attack & Educational Institutes, Transportation \\
\hline $\begin{array}{l}\text { Central American Movement of } \\
\text { Solidarity (MOSCA) }\end{array}$ & Nationalist & 1995 & 1995 & Honduras & Bombing/Explosion & $\begin{array}{l}\text { Government (General), Journalists \& } \\
\text { Media }\end{array}$ \\
\hline Chaotic Attack Front & Left-Wing & 2003 & 2004 & Greece & Bombing/Explosion & Businesses, Government (General) \\
\hline Charlot Jacquelin Militant Front & Nationalist & 1995 & 1995 & Haiti & Hostage Taking & Government (General) \\
\hline Che Guevara Anti-Imperialist Command & Left-Wing & 2005 & - & Argentina & Bombing/Explosion & Businesses, Government (General) \\
\hline Chechen European Network & Religious & 2012 & - & Russia & Bombing/Explosion & Military \\
\hline Children of Fire & Left-Wing & 1996 & 2001 & Greece & Armed Assault, Bombing/Explosion & Businesses, Government (General) \\
\hline $\begin{array}{l}\text { Circle of Lawbreakers-Nuclei Lovers of } \\
\text { Lawlessness-Fighting Minority }\end{array}$ & Left-Wing & 2013 & - & Greece & Bombing/Explosion & Journalists \& Media \\
\hline Cirebon & Religious & 2009 & - & Indonesia & Armed Assault, Bombing/Explosion & $\begin{array}{l}\text { Businesses, Journalists \& Media, Police, } \\
\text { Religious Figures/Institutions }\end{array}$ \\
\hline Citizen's Rights Protection Volunteers & Nationalist & 2009 & 2009 & India & Facility/Infrastructure Attack & Private Citizens \& Property \\
\hline Civil Defense Force (CDF) & Nationalist & 1999 & 2002 & Sierra Leone & Armed Assault & $\begin{array}{l}\text { Private Citizens \& Property, } \\
\text { Terrorists/Non-State Militia }\end{array}$ \\
\hline Civilian Joint Task Force (JTF) & Nationalist & 2013 & - & Nigeria & Facility/Infrastructure Attack & Private Citizens \& Property \\
\hline Clandestini & Nationalist & 1998 & 1999 & France & Bombing/Explosion & $\begin{array}{l}\text { Government (General), Private Citizens } \\
\text { \& Property }\end{array}$ \\
\hline Clandestini Corsi & $\begin{array}{l}\text { Right- } \\
\text { Wing }\end{array}$ & 1999 & 1999 & France & Bombing/Explosion & Government (General) \\
\hline Clandestini Ribelli & Nationalist & 2000 & 2006 & France & Armed Assault & Government (General) \\
\hline Clone of Lega Nord & Nationalist & 2014 & - & Italy & Armed Assault, Hostage Taking & Private Citizens \& Property \\
\hline $\begin{array}{l}\text { Coalition for Militant Action in the Niger } \\
\text { Delta (COMA) }\end{array}$ & Nationalist & 2005 & - & Nigeria & Armed Assault, Hostage Taking & $\begin{array}{l}\text { Military, Private Citizens \& Property, } \\
\text { Utilities }\end{array}$ \\
\hline $\begin{array}{l}\text { Coalition for Unity and Democracy } \\
\text { (CUD) }\end{array}$ & Nationalist & 2006 & - & Ethiopia & Bombing/Explosion & Businesses, Educational Institutes \\
\hline Colombian Patriotic Resistance & Left-Wing & 1999 & 1999 & Colombia & Bombing/Explosion & Businesses, NGO \\
\hline Colonel Karuna Faction & Nationalist & 2004 & 2007 & Sri Lanka & Armed Assault, Bombing/Explosion & $\begin{array}{l}\text { Government (General), Private Citizens } \\
\text { \& Property, Terrorists/Non-State Militia }\end{array}$ \\
\hline $\begin{array}{l}\text { Comando Jaramillista Morelense } 23 \text { de } \\
\text { Mayo }\end{array}$ & Left-Wing & 2004 & 2004 & Mexico & Bombing/Explosion & Businesses, Journalists \& Media \\
\hline Combat 18 & $\begin{array}{l}\text { Right- } \\
\text { Wing }\end{array}$ & 1992 & - & Australia, Russia, United Kingdom & Armed Assault, Bombing/Explosion & $\begin{array}{l}\text { Private Citizens \& Property, Religious } \\
\text { Figures/Institutions }\end{array}$ \\
\hline
\end{tabular}




\begin{tabular}{|c|c|c|c|c|c|c|}
\hline $\begin{array}{l}\text { VEO Name } \\
\end{array}$ & Type & Emerged & Ended & Countries & Type of Attack & Target Type \\
\hline Commando Anarchist Group & Left-Wing & 2003 & 2003 & Greece & Bombing/Explosion & Businesses \\
\hline $\begin{array}{l}\text { Committee for Promotion of } \\
\text { Intransigence }\end{array}$ & Left-Wing & 1993 & 1993 & Greece & Bombing/Explosion & Businesses, Government (General) \\
\hline $\begin{array}{l}\text { Committee for the Security of the } \\
\text { Highways }\end{array}$ & Nationalist & 1998 & 2001 & Israel & Armed Assault & Government (General) \\
\hline Communist Liberation Faction & Left-Wing & 2005 & - & Greece & Bombing/Explosion & $\begin{array}{l}\text { Government (General), Journalists \& } \\
\text { Media }\end{array}$ \\
\hline Communist Party of India-Maoist & Left-Wing & 2004 & - & India & $\begin{array}{l}\text { Armed Assault, Assassination, } \\
\text { Bombing/Explosion, } \\
\text { Facility/Infrastructure Attack, } \\
\text { Hijacking, Hostage Taking, Unarmed } \\
\text { Assault }\end{array}$ & $\begin{array}{l}\text { Businesses, Educational Institutes, } \\
\text { Government (General), Journalists \& } \\
\text { Media, Military, Police, Private Citizens } \\
\text { \& Property, Religious } \\
\text { Figures/Institutions, } \\
\text { Telecommunication, Terrorists/Non- } \\
\text { State Militia, Transportation, Utilities, } \\
\text { Violent Political Party }\end{array}$ \\
\hline Communist Party of India-Marxist & Left-Wing & 2001 & 2001 & India & $\begin{array}{l}\text { Armed Assault, Assassination, } \\
\text { Bombing/Explosion, } \\
\text { Facility/Infrastructure Attack }\end{array}$ & $\begin{array}{l}\text { Educational Institutes, Government } \\
\text { (General), Private Citizens \& Property, } \\
\text { Violent Political Party }\end{array}$ \\
\hline $\begin{array}{l}\text { Communist Party of India-Marxist- } \\
\text { Leninist (CPI-ML) Janashakti }\end{array}$ & Left-Wing & 1992 & - & India, Nepal & $\begin{array}{l}\text { Armed Assault, Assassination, } \\
\text { Bombing/Explosion, Hostage Taking }\end{array}$ & $\begin{array}{l}\text { Government (General), Police, Private } \\
\text { Citizens \& Property, Violent Political } \\
\text { Party }\end{array}$ \\
\hline $\begin{array}{l}\text { Communist Party of Nepal - Unified } \\
\text { Marxist-Leninist (CPN-UML) }\end{array}$ & Left-Wing & 2008 & - & Nepal & Armed Assault & $\begin{array}{l}\text { Private Citizens \& Property, } \\
\text { Terrorists/Non-State Militia }\end{array}$ \\
\hline $\begin{array}{l}\text { Communist Party of Nepal - Unity } \\
\text { Centre-Masal }\end{array}$ & Left-Wing & 2002 & - & Nepal & Unknown & Unknown \\
\hline $\begin{array}{l}\text { Communist Party of Nepal-Maoist } \\
\text { (CPN-M) }\end{array}$ & Left-Wing & 1996 & 2006 & India, Nepal & $\begin{array}{l}\text { Armed Assault, Assassination, } \\
\text { Bombing/Explosion, } \\
\text { Facility/Infrastructure Attack, Hostage } \\
\text { Taking }\end{array}$ & $\begin{array}{l}\text { Airports \& Aircraft, Educational } \\
\text { Institutes, Government (General), } \\
\text { Military, Police, Private Citizens \& } \\
\text { Property, Tourists, Violent Political } \\
\text { Party }\end{array}$ \\
\hline Communist Trade Union Workers & Left-Wing & 1992 & 1992 & India & Unknown & Private Citizens \& Property \\
\hline $\begin{array}{l}\text { Congress of the People's of Ichkeria and } \\
\text { Dagestan }\end{array}$ & Religious & 1996 & 2004 & Russia & Armed Assault, Bombing/Explosion & $\begin{array}{l}\text { Educational Institutes, Military, NGO, } \\
\text { Private Citizens \& Property }\end{array}$ \\
\hline Conscientious Arsonists & Left-Wing & 1997 & 1998 & Greece & $\begin{array}{l}\text { Bombing/Explosion, } \\
\text { Facility/Infrastructure Attack }\end{array}$ & $\begin{array}{l}\text { Businesses, Government (Diplomatic), } \\
\text { Government (General), Journalists \& } \\
\text { Media, Private Citizens \& Property }\end{array}$ \\
\hline $\begin{array}{l}\text { Conspiracy of Cells of Fire (CCF) - } \\
\text { Mexico }\end{array}$ & Left-Wing & 2011 & - & Mexico & Bombing/Explosion & Businesses \\
\hline
\end{tabular}




\begin{tabular}{|c|c|c|c|c|c|c|}
\hline VEO Name & Type & Emerged & Ended & Countries & Type of Attack & $\begin{array}{c}\text { Target Type } \\
\end{array}$ \\
\hline $\begin{array}{l}\text { Conspiracy of Cells of Fire (CCF-FAI- } \\
\text { FRI) }\end{array}$ & Left-Wing & 2008 & - & Greece & $\begin{array}{l}\text { Assassination, Bombing/Explosion, } \\
\text { Facility/Infrastructure Attack }\end{array}$ & $\begin{array}{l}\text { Businesses, Government (Diplomatic), } \\
\text { Government (General), Journalists \& } \\
\text { Media, Police, Private Citizens \& } \\
\text { Property, Transportation, Violent } \\
\text { Political Party }\end{array}$ \\
\hline $\begin{array}{l}\text { Convention of Patriots for Justice and } \\
\text { Peace (CPJP) }\end{array}$ & Nationalist & 2009 & - & The Central African Republic & Armed Assault & Government (General), Military, Police \\
\hline $\begin{array}{l}\text { Cooperative of Hand-Made Fire and } \\
\text { Related Items }\end{array}$ & Left-Wing & 2001 & - & Italy & Bombing/Explosion & Businesses \\
\hline CorCom (Coordination Committee) & Nationalist & 2011 & - & India & Bombing/Explosion & $\begin{array}{l}\text { Businesses, Educational Institutes, } \\
\text { Government (General), Military, Police, } \\
\text { Private Citizens \& Property, } \\
\text { Transportation }\end{array}$ \\
\hline Corsica Nazione & Nationalist & 1992 & - & France & $\begin{array}{l}\text { Armed Assault, Assassination, } \\
\text { Bombing/Explosion }\end{array}$ & $\begin{array}{l}\text { Government (General), Private Citizens } \\
\text { \& Property }\end{array}$ \\
\hline Corsican Patriotic Front (FPC) & Nationalist & 1999 & 1999 & France & Bombing/Explosion & $\begin{array}{l}\text { Businesses, Government (General), } \\
\text { Utilities }\end{array}$ \\
\hline Corsican Revolutionary Armed Forces & Nationalist & 1992 & - & France & Unknown & Unknown \\
\hline Cypriot Nationalists Organization & Nationalist & 2004 & - & Cyprus & Bombing/Explosion & Government (General) \\
\hline Dadullah Front & Nationalist & 2012 & - & Afghanistan & Assassination, Bombing/Explosion & Government (General) \\
\hline Dagestan Liberation Army & Nationalist & 1999 & 2004 & Russia & Bombing/Explosion & Private Citizens \& Property \\
\hline Dagestani Shari'ah Jamaat & Nationalist & 2002 & - & Russia & $\begin{array}{l}\text { Armed Assault, Assassination, } \\
\text { Bombing/Explosion, Hostage Taking }\end{array}$ & $\begin{array}{l}\text { Government (General), Military, Police, } \\
\text { Private Citizens \& Property }\end{array}$ \\
\hline Dario Santillan Command & Left-Wing & 2004 & 2004 & Argentina & Bombing/Explosion & Businesses \\
\hline $\begin{array}{l}\text { David Yau Yau Rebel Group/David Yau } \\
\text { Yau Militia }\end{array}$ & Nationalist & 2010 & - & South Sudan & Armed Assault, Hostage Taking & $\begin{array}{l}\text { Government (Diplomatic), Military, } \\
\text { Private Citizens \& Property }\end{array}$ \\
\hline David's Sword & Religious & 1994 & 1994 & Palestinian State & Assassination & Government (General) \\
\hline $\begin{array}{l}\text { Dawn of Freedom Brigades (Tajammu' } \\
\text { Alwiya Fajr al-Hurriya) }\end{array}$ & Nationalist & 2014 & - & Syria & $\begin{array}{l}\text { Armed Assault, Facility/Infrastructure } \\
\text { Attack }\end{array}$ & $\begin{array}{l}\text { Government (General), Terrorists/Non- } \\
\text { State Militia }\end{array}$ \\
\hline de Fes & Religious & 1994 & 1994 & France, Morocco & Armed Assault & Businesses, Tourists \\
\hline Death Squad of Mujahideen of Iraq & Nationalist & 2004 & - & Iraq & Hostage Taking & $\begin{array}{l}\text { Businesses, Government (General), } \\
\text { Military, Private Citizens \& Property }\end{array}$ \\
\hline Deccan Mujahideen & Religious & 2008 & - & India & $\begin{array}{l}\text { Armed Assault, Bombing/Explosion, } \\
\text { Hijacking, Hostage Taking }\end{array}$ & $\begin{array}{l}\text { Businesses, Maritime, Police, Private } \\
\text { Citizens \& Property, Religious } \\
\text { Figures/Institutions, Tourists, } \\
\text { Transportation }\end{array}$ \\
\hline Defenders of the Nation's Sovereignty & Nationalist & 2013 & - & Madagascar & Bombing/Explosion & Businesses \\
\hline
\end{tabular}




\begin{tabular}{|c|c|c|c|c|c|c|}
\hline VEO Name & Type & Emerged & Ended & Countries & Type of Attack & Target Type \\
\hline Delta Democratic Militia & Nationalist & 2011 & - & Niger & Bombing/Explosion & Government (General) \\
\hline Democratic Front for Renewal (FDR) & Nationalist & 1995 & 1996 & Niger & Unknown & $\begin{array}{l}\text { Private Citizens \& Property, } \\
\text { Transportation }\end{array}$ \\
\hline $\begin{array}{l}\text { Democratic Front for the Central African } \\
\text { People (FDPC) }\end{array}$ & Nationalist & 2014 & - & Cameroon, Central African Republic & Hostage Taking & $\begin{array}{l}\text { Government (Diplomatic), Government } \\
\text { (General), Private Citizens \& Property, } \\
\text { Religious Figures/Institutions }\end{array}$ \\
\hline $\begin{array}{l}\text { Democratic Front for the Liberation of } \\
\text { Rwanda (FDLR) }\end{array}$ & Nationalist & 2007 & - & Congo, Democratic Republic & $\begin{array}{l}\text { Armed Assault, Assassination, } \\
\text { Bombing/Explosion, } \\
\text { Facility/Infrastructure Attack, } \\
\text { Hijacking, Hostage Taking }\end{array}$ & $\begin{array}{l}\text { Airports \& Aircraft, Government } \\
\text { (Diplomatic), Military, NGO, Police, } \\
\text { Private Citizens \& Property, Religious } \\
\text { Figures/Institutions, Transportation }\end{array}$ \\
\hline $\begin{array}{l}\text { Democratic Iraqi Opposition of } \\
\text { Germany }\end{array}$ & Religious & 2002 & 2002 & Germany & Hostage Taking & Government (Diplomatic) \\
\hline $\begin{array}{l}\text { Democratic Movement for the } \\
\text { Liberation of the Eritrean Kunamas } \\
\text { (DMLEK) }\end{array}$ & Nationalist & 2007 & - & Eritrea & $\begin{array}{l}\text { Armed Assault, Bombing/Explosion, } \\
\text { Facility/Infrastructure Attack }\end{array}$ & Government (General), Military \\
\hline Democratic Progressive Party & Nationalist & 1992 & 1992 & Thailand & Unknown & Government (General) \\
\hline Democratic Revolutionary Party (PRD) & Left-Wing & 1995 & 1998 & Mexico & Armed Assault, Hostage Taking & $\begin{array}{l}\text { Government (General), Private Citizens } \\
\text { \& Property, Transportation }\end{array}$ \\
\hline Desert Brigades & Religious & 2012 & - & Mali & Hostage Taking & $\begin{array}{l}\text { Government (General), Private Citizens } \\
\text { \& Property }\end{array}$ \\
\hline Desert Falcons/Liwa Suqur al-Shura & Nationalist & 2013 & - & Iraq, Syria & Armed Assault & Private Citizens \& Property \\
\hline $\begin{array}{l}\text { Deviant Behaviors for the Expansion of } \\
\text { Revolutionary Terrorism International } \\
\text { Revolutionary Front }\end{array}$ & Left-Wing & 2012 & - & Greece & Bombing/Explosion & Businesses \\
\hline Dhi Qar Organization & Nationalist & 2006 & - & Iraq & Armed Assault & Government (General) \\
\hline Dignity for Colombia & Nationalist & 1995 & 1996 & Colombia & Assassination, Hostage Taking & $\begin{array}{l}\text { Businesses, Government (General), } \\
\text { Journalists \& Media, Private Citizens \& } \\
\text { Property }\end{array}$ \\
\hline Dima Halam Daoga (DHD) & Nationalist & 1996 & 2013 & India & $\begin{array}{l}\text { Armed Assault, Bombing/Explosion, } \\
\text { Facility/Infrastructure Attack, Hostage } \\
\text { Taking }\end{array}$ & $\begin{array}{l}\text { Businesses, Government (General), } \\
\text { Military, Police, Private Citizens \& } \\
\text { Property, Transportation }\end{array}$ \\
\hline $\begin{array}{l}\text { Dima National Democratic Front } \\
\text { (DNDF) }\end{array}$ & Nationalist & 2010 & - & India & Hostage Taking & Private Citizens \& Property \\
\hline Diraa al-Shahbaa Rebel Brigade & Nationalist & 2012 & - & Syria & Assassination & Journalists \& Media \\
\hline Direh al-Aasmeh/Shield of Damascus & Religious & 2013 & - & Syria & Bombing/Explosion & Military \\
\hline Divine Wrath Brigades & Religious & 2004 & - & Iraq & Hostage Taking & Military \\
\hline Diyala Salvation Council & Religious & 2007 & - & Iraq & Armed Assault & Terrorists/Non-State Militia \\
\hline
\end{tabular}




\begin{tabular}{|c|c|c|c|c|c|c|}
\hline VEO Name & Type & Emerged & Ended & Countries & Type of Attack & Target Type \\
\hline Donetsk People's Republic & Nationalist & 2014 & - & Ukraine & $\begin{array}{l}\text { Armed Assault, Bombing/Explosion, } \\
\text { Facility/Infrastructure Attack, Hostage } \\
\text { Taking, Unarmed Assault }\end{array}$ & $\begin{array}{l}\text { Airports \& Aircraft, Businesses, } \\
\text { Educational Institutes, Food or Water } \\
\text { Supply, Government (Diplomatic), } \\
\text { Government (General), Journalists \& } \\
\text { Media, Military, NGO, Police, Private } \\
\text { Citizens \& Property, Religious } \\
\text { Figures/Institutions, } \\
\text { Telecommunication, Terrorists/Non- } \\
\text { State Militia, Transportation, Utilities }\end{array}$ \\
\hline Draa El Mizan Seriat & Religious & 2002 & 2002 & Algeria & Armed Assault, Hostage Taking & Businesses, Military \\
\hline Dwekh Nawsha/al-Fedayoon & Nationalist & 2014 & - & Iraq & Armed Assault & Terrorists/Non-State Militia \\
\hline Earth Liberation Front (ELF) & Left-Wing & 1992 & - & $\begin{array}{l}\text { Canada, Chile, Greece, Mexico, United } \\
\text { Kingdom, United States }\end{array}$ & $\begin{array}{l}\text { Bombing/Explosion, } \\
\text { Facility/Infrastructure Attack }\end{array}$ & Businesses \\
\hline East Turkestan Liberation Tigers & Nationalist & 1998 & - & China, Pakistan, Turkey & Bombing/Explosion & Private Citizens \& Property \\
\hline East Turkistan Liberation Organization & Nationalist & 1996 & 1999 & China, Kyrgyzstan, Turkey & Armed Assault, Assassination & $\begin{array}{l}\text { Businesses, Government (Diplomatic), } \\
\text { Private Citizens \& Property }\end{array}$ \\
\hline $\begin{array}{l}\text { Eastern Turkistan Islamic Movement } \\
\text { (ETIM) }\end{array}$ & Religious & 1997 & - & $\begin{array}{l}\text { Afghanistan, China, Kyrgyzstan, } \\
\text { Pakistan, Uzbekistan }\end{array}$ & $\begin{array}{l}\text { Armed Assault, Bombing/Explosion, } \\
\text { Facility/Infrastructure Attack, Unarmed } \\
\text { Assault }\end{array}$ & $\begin{array}{l}\text { Government (Diplomatic), Government } \\
\text { (General), Police, Private Citizens \& } \\
\text { Property, Transportation }\end{array}$ \\
\hline Ecuadorian National Socialism (NSE) & $\begin{array}{l}\text { Right- } \\
\text { Wing }\end{array}$ & 2001 & 2003 & Ecuador & Armed Assault, Bombing/Explosion & $\begin{array}{l}\text { Government (General), NGO, } \\
\text { Terrorists/Non-State Militia }\end{array}$ \\
\hline Ecuadorian Rebel Force & Religious & 2001 & 2001 & Ecuador & Bombing/Explosion & $\begin{array}{l}\text { Government (General), } \\
\text { Telecommunication, Utilities }\end{array}$ \\
\hline Egbema National Front & Nationalist & 2003 & 2003 & Nigeria & Hostage Taking & Businesses \\
\hline Egypt's Mujahideen & Religious & 2005 & - & Egypt & Bombing/Explosion & Military \\
\hline Ejercito del Pueblo en Armas & Left-Wing & 2002 & - & Venezuela & Bombing/Explosion, Hostage Taking & NGO, Religious Figures/Institutions \\
\hline $\begin{array}{l}\text { Ejercito Revolucionario } \\
\text { Guevarista/Guevarista Revolutionary } \\
\text { Army }\end{array}$ & Left-Wing & 1992 & 2008 & Colombia & Unknown & NGO, Private Citizens \& Property \\
\hline El Farouk Brigade & Religious & 2008 & - & Algeria & Armed Assault, Bombing/Explosion & Military, NGO, Utilities \\
\hline El-Feth Katibat & Religious & 2006 & 2006 & Algeria & Bombing/Explosion & Military \\
\hline English Defence League (EDL) & Left-Wing & 2009 & - & United Kingdom & $\begin{array}{l}\text { Armed Assault, Facility/Infrastructure } \\
\text { Attack }\end{array}$ & $\begin{array}{l}\text { Government (General), Religious } \\
\text { Figures/Institutions, Terrorists/Non- } \\
\text { State Militia }\end{array}$ \\
\hline $\begin{array}{l}\text { Ethiopian People's Patriotic Front } \\
\text { (EPPF) }\end{array}$ & Left-Wing & 2000 & - & Estonia, Ethiopia, Sudan & Armed Assault, Bombing/Explosion & Military \\
\hline Etnocacerista Movement & $\begin{array}{l}\text { Right- } \\
\text { Wing }\end{array}$ & 2000 & 2005 & Peru & Armed Assault, Hostage Taking & Police, Utilities \\
\hline
\end{tabular}




\begin{tabular}{|c|c|c|c|c|c|c|}
\hline VEO Name & Type & Emerged & Ended & Countries & Type of Attack & Target Type \\
\hline $\begin{array}{l}\text { Expedition in Revenge of Our People in } \\
\text { Baniyas }\end{array}$ & Nationalist & 2013 & - & Syria & Armed Assault & Military \\
\hline $\begin{array}{l}\text { External Security Organization of } \\
\text { Hezbollah (ESO)/Islamic Jihad } \\
\text { Organization }\end{array}$ & Religious & 1993 & - & Argentina, Lebanon & Bombing/Explosion & Businesses, Government (Diplomatic) \\
\hline Face to Face & Nationalist & 2002 & 2002 & Haiti & Facility/Infrastructure Attack & Government (General) \\
\hline Fallujah Mujahideen & Religious & 2000 & 2004 & Iraq & Hostage Taking & Private Citizens \& Property \\
\hline Fanmi Lavalas & Left-Wing & 2002 & 2002 & Haiti & Assassination & Government (General) \\
\hline Fansara 110/New Forces & Nationalist & 2006 & - & Cote D'Ivoire & Armed Assault, Hostage Taking & $\begin{array}{l}\text { Government (General), Private Citizens } \\
\text { \& Property }\end{array}$ \\
\hline Farighan & Nationalist & 1992 & 1992 & Ghana & Bombing/Explosion & $\begin{array}{l}\text { Airports \& Aircraft, Government } \\
\text { (General), Journalists \& Media }\end{array}$ \\
\hline Farouq Brigade & Religious & 2012 & - & Syria, Turkey & Armed Assault, Bombing/Explosion & Government (General), Military \\
\hline Farzandan-e-Millat & Nationalist & 2005 & 2005 & India & Armed Assault & Transportation \\
\hline Fatah al-Islam & Religious & 2006 & - & Lebanon, Palestinian State, Switzerland & $\begin{array}{l}\text { Armed Assault, Assassination, } \\
\text { Bombing/Explosion, } \\
\text { Facility/Infrastructure Attack }\end{array}$ & $\begin{array}{l}\text { Government (Diplomatic), Military, } \\
\text { NGO, Terrorists/Non-State Militia, } \\
\text { Transportation }\end{array}$ \\
\hline Fatah Hawks & Nationalist & 2001 & 2001 & Palestinian State & $\begin{array}{l}\text { Armed Assault, Assassination, Hostage } \\
\text { Taking }\end{array}$ & $\begin{array}{l}\text { Businesses, Educational Institutes, } \\
\text { Journalists \& Media, Police, Private } \\
\text { Citizens \& Property }\end{array}$ \\
\hline Fatiheen Army & Religious & 2011 & - & Iraq & Armed Assault, Bombing/Explosion & Military \\
\hline February 12 Movement & Left-Wing & 2012 & - & Greece & Bombing/Explosion & Government (General), Transportation \\
\hline February 14 Movement & Nationalist & 2011 & - & Bahrain & Bombing/Explosion & $\begin{array}{l}\text { Businesses, Educational Institutes, } \\
\text { Government (General), Military, Police, } \\
\text { Private Citizens \& Property }\end{array}$ \\
\hline February 17 Martyrs Brigade & Nationalist & 2014 & - & Libya & Bombing/Explosion & $\begin{array}{l}\text { Private Citizens \& Property, } \\
\text { Terrorists/Non-State Militia }\end{array}$ \\
\hline Fedayeen Imam Mahdi & Religious & 2003 & - & Pakistan & Armed Assault, Assassination & Government (General) \\
\hline Fedayeen Saddam & Nationalist & 1995 & 2003 & Iraq & Armed Assault & Military, Private Citizens \& Property \\
\hline Fedey-e-Mahaz & Nationalist & 2013 & - & Afghanistan & Armed Assault, Assassination & $\begin{array}{l}\text { Journalists \& Media, NGO, } \\
\text { Terrorists/Non-State Militia }\end{array}$ \\
\hline Fighters for Freedom & Left-Wing & 1996 & 2000 & Greece & Bombing/Explosion & Government (General) \\
\hline Fighting Guerrilla Formation (MAS) & Left-Wing & 1996 & 1998 & Greece & Bombing/Explosion & $\begin{array}{l}\text { Airports \& Aircraft, Educational } \\
\text { Institutes, Government (Diplomatic), } \\
\text { Government (General) }\end{array}$ \\
\hline Fighting Guerrillas of May & Left-Wing & 1998 & 1998 & Greece & Bombing/Explosion & Airports \& Aircraft, Businesses \\
\hline
\end{tabular}




\begin{tabular}{|c|c|c|c|c|c|c|}
\hline VEO Name & Type & Emerged & Ended & Countries & Type of Attack & Target Type \\
\hline Fires of Hell & Left-Wing & 2004 & 2004 & Greece & Bombing/Explosion & Religious Figures/Institutions \\
\hline For a Revolutionary Perspective & Left-Wing & 2001 & 2001 & Switzerland & Bombing/Explosion & $\begin{array}{l}\text { Airports \& Aircraft, Government } \\
\text { (Diplomatic) }\end{array}$ \\
\hline Forces Armees Congolaises (FAC) & Nationalist & 1997 & 1998 & Congo, Democratic Republic & Armed Assault & Private Citizens \& Property \\
\hline $\begin{array}{l}\text { Forces for the Defense of Democracy } \\
\text { (FDD) }\end{array}$ & Nationalist & 1993 & 2003 & $\begin{array}{l}\text { Burundi, Congo, Democratic Republic, } \\
\text { Tanzania }\end{array}$ & $\begin{array}{l}\text { Armed Assault, Bombing/Explosion, } \\
\text { Hostage Taking }\end{array}$ & $\begin{array}{l}\text { Government (General), NGO, Private } \\
\text { Citizens \& Property, Religious } \\
\text { Figures/Institutions, Terrorists/Non- } \\
\text { State Militia }\end{array}$ \\
\hline $\begin{array}{l}\text { Forces for the Unification of the Central } \\
\text { African Republic (FIRCA) }\end{array}$ & Nationalist & 2008 & - & The Central African Republic & Armed Assault & Private Citizens \& Property \\
\hline Forces Nouvelles & Nationalist & 2002 & - & Cote D'Ivoire & Unknown & Unknown \\
\hline $\begin{array}{l}\text { Forum Antisipasi Kegiatan Pemurtadan } \\
\text { (FAKTA) }\end{array}$ & Religious & 1998 & - & Indonesia & Assassination, Bombing/Explosion & Religious Figures/Institutions \\
\hline Forum for the Restoration of Democracy & Nationalist & 1997 & 1997 & Kenya & Armed Assault, Unarmed Assault & $\begin{array}{l}\text { Government (General), Private Citizens } \\
\text { \& Property }\end{array}$ \\
\hline $\begin{array}{l}\text { Forum for Unity, Democracy, and } \\
\text { Justice (PFDK/UNTAS) }\end{array}$ & Nationalist & 1998 & - & Indonesia & Armed Assault & Private Citizens \& Property \\
\hline Free All Political Prisoners in Holland & Left-Wing & 1992 & 1992 & Netherlands & Unknown & Military \\
\hline Free Balochistan Army (FBA) & Nationalist & 2014 & - & Pakistan & Bombing/Explosion & Government (General) \\
\hline Free Libya Martyrs Brigade & Nationalist & 2015 & - & Libya & Bombing/Explosion & Military, Private Citizens \& Property \\
\hline Free Network South & $\begin{array}{l}\text { Right- } \\
\text { Wing }\end{array}$ & 2013 & - & Germany & Facility/Infrastructure Attack & Unknown \\
\hline Free Salafist Group (GSL) & Religious & 2003 & - & Algeria & Hostage Taking & Private Citizens \& Property, Tourists \\
\hline Free Sunnis of Baalbek Brigade & Religious & 2013 & - & Lebanon & $\begin{array}{l}\text { Armed Assault, Assassination, } \\
\text { Bombing/Explosion, Hostage Taking }\end{array}$ & Military, Violent Political Party \\
\hline Free Syrian Army (FSA) & Left-Wing & 2011 & & Syria, Turkey & $\begin{array}{l}\text { Armed Assault, Assassination, } \\
\text { Bombing/Explosion, Hostage Taking }\end{array}$ & $\begin{array}{l}\text { Airports \& Aircraft, Businesses, } \\
\text { Educational Institutes, Government } \\
\text { (Diplomatic), Government (General), } \\
\text { Journalists \& Media, Military, Private } \\
\text { Citizens \& Property, Religious } \\
\text { Figures/Institutions, Terrorists/Non- } \\
\text { State Militia, Utilities }\end{array}$ \\
\hline Free Vietnam Revolutionary Group & Nationalist & 2001 & 2001 & $\begin{array}{l}\text { Philippines, Thailand, United States, } \\
\text { Vietnam }\end{array}$ & Bombing/Explosion & $\begin{array}{l}\text { Government (Diplomatic), Government } \\
\text { (General) }\end{array}$ \\
\hline Freedom Eagles of Africa & Nationalist & 2009 & - & Central African Republic, South Sudan & Hostage Taking & NGO \\
\hline Freedom for Mumia Abu-Jamal & Left-Wing & 1999 & 1999 & Switzerland & Bombing/Explosion & Airports \& Aircraft, Businesses, NGO \\
\hline
\end{tabular}




\begin{tabular}{|c|c|c|c|c|c|c|}
\hline VEO Name & Type & Emerged & Ended & Countries & $\begin{array}{c}\text { Type of Attack } \\
\end{array}$ & $\begin{array}{c}\text { Target Type } \\
\end{array}$ \\
\hline Freital Group & $\begin{array}{l}\text { Right- } \\
\text { Wing }\end{array}$ & 2015 & - & Germany & Assassination, Bombing/Explosion & $\begin{array}{l}\text { Government (General), Private Citizens } \\
\text { \& Property }\end{array}$ \\
\hline French Armed Islamic Front & Religious & 2004 & - & France & Bombing/Explosion & Government (Diplomatic) \\
\hline Friends of Loukanikos & Left-Wing & 2012 & - & Greece & $\begin{array}{l}\text { Bombing/Explosion, } \\
\text { Facility/Infrastructure Attack }\end{array}$ & Government (Diplomatic) \\
\hline Friends of the Earth (FAI) & $\begin{array}{l}\text { Left-Wing } \\
\end{array}$ & 2012 & - & Argentina & Bombing/Explosion & $\begin{array}{l}\text { Businesses, Government (Diplomatic), } \\
\text { Police, Private Citizens \& Property }\end{array}$ \\
\hline Friendship Society & Nationalist & 1999 & 1999 & Greece & Bombing/Explosion & Businesses \\
\hline $\begin{array}{l}\text { Front for Alternation and Concord in } \\
\text { Chad (FACT) }\end{array}$ & Nationalist & 2016 & - & Chad, Libya & Armed Assault & Military \\
\hline $\begin{array}{l}\text { Front for the Liberation of the Golan } \\
\text { Heights/Committees for the Liberation } \\
\text { of Golan Heights }\end{array}$ & Religious & 2006 & - & Syria & Armed Assault, Hostage Taking & Military \\
\hline $\begin{array}{l}\text { Front Pembela Islam (FPI)/Front for } \\
\text { Defenders of Islam }\end{array}$ & Religious & 1997 & - & Indonesia & Armed Assault, Bombing/Explosion & $\begin{array}{l}\text { Businesses, NGO, Police, Religious } \\
\text { Figures/Institutions }\end{array}$ \\
\hline $\begin{array}{l}\text { Front Pemuda Islam Solo (FPIS)/Solo } \\
\text { Islamic Youth Front }\end{array}$ & Religious & 1999 & - & Afghanistan, Indonesia & Armed Assault & $\begin{array}{l}\text { Businesses, Government (General), } \\
\text { Private Citizens \& Property, Religious } \\
\text { Figures/Institutions }\end{array}$ \\
\hline $\begin{array}{l}\text { Fuerzas Armadas de Revolucionarias de } \\
\text { Ecuador (FARE) }\end{array}$ & Left-Wing & 2000 & 2002 & Ecuador & $\begin{array}{l}\text { Assassination, Bombing/Explosion, } \\
\text { Hostage Taking }\end{array}$ & $\begin{array}{l}\text { Businesses, Government (General), } \\
\text { Military, Utilities }\end{array}$ \\
\hline $\begin{array}{l}\text { Fuerzas Armadas Revolucionarias del } \\
\text { Pueblo (FARP) }\end{array}$ & Left-Wing & 2000 & 2001 & Mexico & Armed Assault, Bombing/Explosion & Businesses, Police \\
\hline $\begin{array}{l}\text { Fuerzas Autonomicas y Destructivas } \\
\text { Leon Czolgoscz }\end{array}$ & Left-Wing & 2006 & 2006 & Chile & Bombing/Explosion & Government (General) \\
\hline Galician Resistance (REGA) & Nationalist & 2005 & - & Spain & Bombing/Explosion & $\begin{array}{l}\text { Businesses, Government (General), } \\
\text { Police }\end{array}$ \\
\hline Ganda Koi & Nationalist & 1994 & 1994 & Mali & Unarmed Assault & Private Citizens \& Property \\
\hline Gangs of Conscience & Left-Wing & 2008 & - & Greece & Bombing/Explosion & Police \\
\hline Garo National Liberation Army (GNLA) & Nationalist & 2009 & - & India & $\begin{array}{l}\text { Armed Assault, Assassination, } \\
\text { Bombing/Explosion, } \\
\text { Facility/Infrastructure Attack, Hostage } \\
\text { Taking, Unarmed Assault }\end{array}$ & $\begin{array}{l}\text { Businesses, Educational Institutes, } \\
\text { Government (General), Military, Police, } \\
\text { Private Citizens \& Property, } \\
\text { Terrorists/Non-State Militia, } \\
\text { Transportation, Utilities }\end{array}$ \\
\hline Georgian Power & $\begin{array}{l}\text { Right- } \\
\text { Wing }\end{array}$ & 2014 & - & Georgia & Armed Assault & Private Citizens \& Property \\
\hline German Taliban Mujahidin (DTM) & Religious & 2009 & - & Austria, Germany & Armed Assault, Hostage Taking & Government (General) \\
\hline Ghazi Force & Religious & 2008 & - & Palestinian State & Bombing/Explosion & Government (General), Military, NGO \\
\hline
\end{tabular}

\section{9}




\begin{tabular}{|c|c|c|c|c|c|c|}
\hline VEO Name & Type & Emerged & Ended & Countries & Type of Attack & Target Type \\
\hline Gholam Yahya Akbari Brigade & Nationalist & 2009 & - & Afghanistan & Bombing/Explosion, Hostage Taking & Military, NGO \\
\hline Ghuraba al-Sham & Nationalist & 2003 & - & Syria & Armed Assault & Government (General), Military \\
\hline Global Intifada & Left-Wing & 2002 & - & Sweden & $\begin{array}{l}\text { Armed Assault, Facility/Infrastructure } \\
\text { Attack }\end{array}$ & Businesses, Government (Diplomatic) \\
\hline God's Army & Nationalist & 1997 & 2001 & Myanmar, Burma, Thailand & Hijacking, Hostage Taking & $\begin{array}{l}\text { Businesses, Private Citizens \& Property, } \\
\text { Transportation }\end{array}$ \\
\hline God's Oppressed Army & Religious & 1992 & 1992 & Kenya, Nigeria & Bombing/Explosion & Government (General) \\
\hline Gora Euskadi Askatuta & Nationalist & 2002 & - & France & Bombing/Explosion & Police \\
\hline Greek Anarchists' Union & Left-Wing & 1992 & 1992 & Greece & Armed Assault & Government (General), Police \\
\hline Green Battalion & Religious & 2013 & - & Syria & Armed Assault & $\begin{array}{l}\text { Government (General), Violent Political } \\
\text { Party }\end{array}$ \\
\hline Green Berets - Bosnia & Nationalist & 1992 & 1992 & Bosnia and Herzegovina & Unknown & Unknown \\
\hline $\begin{array}{l}\text { Group for Social Resistance to the State } \\
\text { Mechanism }\end{array}$ & Left-Wing & 2004 & 2004 & Greece & Bombing/Explosion & Government (General) \\
\hline $\begin{array}{l}\text { Group for the Promotion of Virtue and } \\
\text { the Prevention of Vice }\end{array}$ & Nationalist & 2005 & - & Iraq & Hostage Taking & Private Citizens \& Property \\
\hline $\begin{array}{l}\text { Group of Guerilla Combatants of Jose } \\
\text { Maria Morelos y Pavon }\end{array}$ & Left-Wing & 2001 & - & Mexico & Bombing/Explosion, Hostage Taking & Businesses, Private Citizens \& Property \\
\hline Group of Popular Combatants & Left-Wing & 1994 & - & Ecuador & Bombing/Explosion & Private Citizens \& Property \\
\hline Group Revolutionary Reconstruction & Left-Wing & 2003 & 2003 & Germany & Bombing/Explosion & Police \\
\hline Guadalcanal Liberation Army & Nationalist & 1996 & 1999 & Solomon Islands & Armed Assault & Private Citizens \& Property \\
\hline Guadalcanal Liberation Front (GLF) & Nationalist & 2002 & - & Solomon Islands & Assassination & Government (General) \\
\hline Guevarista Revolutionary Army & Left-Wing & 1993 & - & Colombia & Assassination & $\begin{array}{l}\text { Government (General), NGO, Private } \\
\text { Citizens \& Property }\end{array}$ \\
\hline Hafiz Gul Bahadar Group & Religious & 2007 & & Afghanistan, Pakistan & $\begin{array}{l}\text { Armed Assault, Bombing/Explosion, } \\
\text { Hostage Taking }\end{array}$ & Government (General), Military \\
\hline Haftar Militia/Libyan National Army & Nationalist & 2014 & - & Libya & $\begin{array}{l}\text { Armed Assault, Assassination, } \\
\text { Bombing/Explosion, Hostage Taking }\end{array}$ & $\begin{array}{l}\text { Businesses, Government (General), } \\
\text { Maritime, Military, Private Citizens \& } \\
\text { Property, Terrorists/Non-State Militia }\end{array}$ \\
\hline Haika/Segi & Nationalist & 2001 & 2001 & Spain & Bombing/Explosion, Unarmed Assault & $\begin{array}{l}\text { Private Citizens \& Property, } \\
\text { Transportation }\end{array}$ \\
\hline Haji Namdar Group & Religious & 2008 & - & Afghanistan, Pakistan & Armed Assault, Bombing/Explosion & Military, Private Citizens \& Property \\
\hline Haji Omar Group & Religious & 2001 & - & Afghanistan, Pakistan & $\begin{array}{l}\text { Armed Assault, Assassination, Hostage } \\
\text { Taking }\end{array}$ & Military \\
\hline Hajong United Liberation Army & Nationalist & 2004 & 2004 & India & Armed Assault, Bombing/Explosion & Government (General) \\
\hline
\end{tabular}




\begin{tabular}{|c|c|c|c|c|c|c|}
\hline VEO Name & Type & Emerged & Ended & Countries & Type of Attack & Target Type \\
\hline Halqa-e-Mehsud & Religious & 2014 & - & Pakistan & $\begin{array}{l}\text { Armed Assault, Assassination, } \\
\text { Bombing/Explosion, Hostage Taking }\end{array}$ & $\begin{array}{l}\text { Businesses, Educational Institutes, } \\
\text { Government (General), Military, Police, } \\
\text { Private Citizens \& Property, } \\
\text { Terrorists/Non-State Militia }\end{array}$ \\
\hline Hamas Iraq & Religious & 2007 & - & Iraq & Armed Assault, Bombing/Explosion & Military \\
\hline Hamas Turabe & Religious & 1992 & 1995 & Bosnia and Herzegovina & Unknown & Unknown \\
\hline Hamawand Tribe & Nationalist & 1992 & 1992 & Turkey & Armed Assault & Violent Political Party \\
\hline HaMered/The Revolt & Nationalist & 2013 & - & Israel, Palestinian State & Bombing/Explosion & $\begin{array}{l}\text { Businesses, Private Citizens \& Property, } \\
\text { Religious Figures/Institutions }\end{array}$ \\
\hline Hamze & Religious & 1996 & - & Bosnia and Herzegovina & Unknown & Unknown \\
\hline Hanchongryun & Left-Wing & 1996 & 1996 & Korea, Republic of & Unarmed Assault & Police \\
\hline $\begin{array}{l}\text { Haqiqi Muthida Quamin Movement } \\
\text { (MQM-H) }\end{array}$ & Nationalist & 1992 & - & Pakistan, United Kingdom & $\begin{array}{l}\text { Armed Assault, Assassination, Hostage } \\
\text { Taking }\end{array}$ & Government (General) \\
\hline Harakat al-Abdal & Religious & 2014 & - & Iraq & Armed Assault & Terrorists/Non-State Militia \\
\hline Harakat al-Nujaba & Religious & 2016 & - & Iraq & Bombing/Explosion & Government (Diplomatic) \\
\hline Harakat al-Shuhada'a al-Islamiyah & Religious & 1996 & - & Libya & Assassination & Government (Diplomatic) \\
\hline Harakat Ansar Iran (HAI) & Religious & 2012 & - & Iran & Bombing/Explosion & Military, Religious Figures/Institutions \\
\hline Harakat Hazm & Nationalist & 2014 & - & Syria & Armed Assault, Bombing/Explosion & $\begin{array}{l}\text { Government (General), Terrorists/Non- } \\
\text { State Militia }\end{array}$ \\
\hline Harakat Hizb Allah al-Nujaba & Religious & 2013 & - & Iraq, Syria & Armed Assault & Unknown \\
\hline Harakat ul-Ansar & Nationalist & 1993 & 2002 & $\begin{array}{l}\text { Bosnia and Herzegovina, India, } \\
\text { Myanmar, Burma, Pakistan, Tajikistan }\end{array}$ & $\begin{array}{l}\text { Armed Assault, Bombing/Explosion, } \\
\text { Hijacking, Hostage Taking }\end{array}$ & $\begin{array}{l}\text { Airports \& Aircraft, Military, Police, } \\
\text { Private Citizens \& Property, Tourists }\end{array}$ \\
\hline $\begin{array}{l}\text { Harakat ul-Jihad-i-Islami } \\
\text { (HUJI)/Harakat ul-Jihad-i-Islami- } \\
\text { Bangladesh (HUJI-B) }\end{array}$ & Religious & 1992 & - & Bangladesh, India, Pakistan & $\begin{array}{l}\text { Armed Assault, Assassination, } \\
\text { Bombing/Explosion }\end{array}$ & $\begin{array}{l}\text { Businesses, Educational Institutes, } \\
\text { Government (General), Police, Private } \\
\text { Citizens \& Property, Religious } \\
\text { Figures/Institutions, Transportation }\end{array}$ \\
\hline Harakat ul-Mujahidin al-Almi (HuMA) & Religious & 2002 & 2002 & Pakistan & Bombing/Explosion & $\begin{array}{l}\text { Businesses, Government (Diplomatic), } \\
\text { Government (General), Private Citizens } \\
\text { \& Property }\end{array}$ \\
\hline Harakat-i-Inqilahi-i-Islami & Religious & 1992 & 1992 & Afghanistan & Assassination & NGO \\
\hline Harakatul-Muhajiriin & Religious & 2009 & - & Cameroon, Nigeria & Armed Assault, Bombing/Explosion & Military, Police \\
\hline $\begin{array}{l}\text { Hashid Shaabi (Popular Mobilisation } \\
\text { Committees) }\end{array}$ & Nationalist & 2014 & - & Iraq & Armed Assault, Bombing/Explosion & Terrorists/Non-State Militia \\
\hline Hasmoneans & Religious & 1992 & 1992 & Israel, Palestinian State & Armed Assault & Government (General) \\
\hline $\begin{array}{l}\text { Hassam (Movement of Egypt's } \\
\text { Forearms) }\end{array}$ & Religious & 2016 & - & Egypt & $\begin{array}{l}\text { Armed Assault, Assassination, } \\
\text { Bombing/Explosion }\end{array}$ & $\begin{array}{l}\text { Government (General), Police, Religious } \\
\text { Figures/Institutions }\end{array}$ \\
\hline
\end{tabular}




\begin{tabular}{|c|c|c|c|c|c|c|}
\hline $\begin{array}{l}\text { VEO Name } \\
\end{array}$ & Type & Emerged & Ended & Countries & Type of Attack & Target Type \\
\hline $\begin{array}{l}\text { Haut Conseil pour I'Unite de I'Azawad } \\
\text { (HCUA) }\end{array}$ & Nationalist & 2013 & - & Mali & Unknown & Military \\
\hline $\begin{array}{l}\text { Hekla Reception Committee-Initiative } \\
\text { for More Social Eruptions }\end{array}$ & Left-Wing & 2011 & - & Germany & Bombing/Explosion & Transportation \\
\hline Helwan Brigades & Nationalist & 2014 & - & Egypt & Armed Assault & Military, Police \\
\hline $\begin{array}{l}\text { Hezb-e-Azadi-ye Afghanistan/Freedom } \\
\text { Party of Afghanistan }\end{array}$ & Nationalist & 1992 & - & Afghanistan & Armed Assault, Bombing/Explosion & Government (General) \\
\hline $\begin{array}{l}\text { Hezb-e-Wahdat-e-Islami-yi } \\
\text { Afghanistan/Islamic Unity Party in } \\
\text { Afghanistan }\end{array}$ & Religious & 1992 & 1992 & Afghanistan & $\begin{array}{l}\text { Armed Assault, Bombing/Explosion, } \\
\text { Hostage Taking }\end{array}$ & $\begin{array}{l}\text { Government (General), Journalists \& } \\
\text { Media, Religious Figures/Institutions, } \\
\text { Terrorists/Non-State Militia }\end{array}$ \\
\hline Hikmatui Zihad & Religious & 2004 & 2004 & Bangladesh & $\begin{array}{l}\text { Armed Assault, Assassination, } \\
\text { Bombing/Explosion }\end{array}$ & Government (General) \\
\hline Hill Students Council & Nationalist & 1992 & 1992 & Bangladesh & Assassination & $\begin{array}{l}\text { Educational Institutes, Government } \\
\text { (General) }\end{array}$ \\
\hline $\begin{array}{l}\text { Hindu Sena Rashtriya Sangh Party } \\
\text { (HSRS) }\end{array}$ & $\begin{array}{l}\text { Right- } \\
\text { Wing }\end{array}$ & 2000 & - & India, Pakistan & Bombing/Explosion & Businesses, Private Citizens \& Property \\
\hline Hizballah al-Abrar & Religious & 2014 & - & Iran, Syria & Armed Assault & Terrorists/Non-State Militia \\
\hline Hizb-al-Tahrir al-Islami (HT) & Religious & 1995 & - & $\begin{array}{l}\text { Bangladesh, China, Germany, } \\
\text { Kazakhstan, Kyrgyzstan, Tajikistan, } \\
\text { Turkmenistan, Uzbekistan }\end{array}$ & Armed Assault, Bombing/Explosion & $\begin{array}{l}\text { Educational Institutes, Government } \\
\text { (General), Transportation }\end{array}$ \\
\hline Hizbul al-Islam & Religious & 2008 & - & Somalia & $\begin{array}{l}\text { Armed Assault, Bombing/Explosion, } \\
\text { Hostage Taking }\end{array}$ & $\begin{array}{l}\text { Journalists \& Media, NGO, Private } \\
\text { Citizens \& Property, } \\
\text { Telecommunication, Terrorists/Non- } \\
\text { State Militia }\end{array}$ \\
\hline Hizbul Islam & Nationalist & 2009 & - & Somalia & Armed Assault & Government (General) \\
\hline Hizbullah Front & Religious & 1999 & - & Indonesia & Armed Assault, Unarmed Assault & Businesses, Private Citizens \& Property \\
\hline Hizb-ut-Tahrir Bangladesh & Religious & 2001 & - & Bangladesh & Assassination, Bombing/Explosion & Government (General) \\
\hline Hizb-ut-Towhid (HUT) & Religious & 1995 & - & $\begin{array}{l}\text { Bangladesh, India, Malaysia, United } \\
\text { Arab Emirates, United States }\end{array}$ & Unknown & $\begin{array}{l}\text { Private Citizens \& Property, Religious } \\
\text { Figures/Institutions }\end{array}$ \\
\hline $\begin{array}{l}\text { Hmar People's Convention-Democracy } \\
\text { (HPC-D) }\end{array}$ & Nationalist & 1995 & - & Bangladesh, India & $\begin{array}{l}\text { Armed Assault, Assassination, } \\
\text { Bombing/Explosion }\end{array}$ & $\begin{array}{l}\text { Businesses, Government (General), } \\
\text { Police, Private Citizens \& Property }\end{array}$ \\
\hline Hofstad Network & Religious & 2002 & 2004 & Netherlands & Assassination & $\begin{array}{l}\text { Government (General), Private Citizens } \\
\text { \& Property }\end{array}$ \\
\hline Holders of the Black Banners & Religious & 2004 & 2004 & Iraq & Hostage Taking & Businesses, Government (General) \\
\hline Holy Jihad Brigades & Religious & 2006 & - & India & Hostage Taking & Government (General), Military \\
\hline Houthi Movement & Religious & 1997 & - & Yemen & Armed Assault, Bombing/Explosion & $\begin{array}{l}\text { Government (General), Terrorists/Non- } \\
\text { State Militia, Utilities }\end{array}$ \\
\hline
\end{tabular}




\begin{tabular}{|c|c|c|c|c|c|c|}
\hline VEO Name & Type & Emerged & Ended & Countries & Type of Attack & Target Type \\
\hline Hungarian Skin Head Group & $\begin{array}{l}\text { Right- } \\
\text { Wing }\end{array}$ & 1992 & 1992 & Hungary & Unknown & Government (Diplomatic) \\
\hline Huria Kristen Batak Protestant & Religious & 1995 & 1995 & Indonesia & $\begin{array}{l}\text { Armed Assault, Facility/Infrastructure } \\
\text { Attack }\end{array}$ & Religious Figures/Institutions \\
\hline Husayn Ubayyat Martyr's Brigades & Religious & 2000 & - & Palestinian State & Armed Assault & Private Citizens \& Property \\
\hline $\begin{array}{l}\text { Hynniewtrep National Liberation } \\
\text { Council (HNLC) }\end{array}$ & Nationalist & 1992 & 2010 & Bangladesh, India & $\begin{array}{l}\text { Armed Assault, Bombing/Explosion, } \\
\text { Hostage Taking }\end{array}$ & $\begin{array}{l}\text { Educational Institutes, Police, Private } \\
\text { Citizens \& Property }\end{array}$ \\
\hline Hynniewtrep State Democratic Front & Nationalist & 1992 & - & Bangladesh & Unknown & Unknown \\
\hline Iconoclasts & Religious & 2004 & 2004 & Italy & Bombing/Explosion & Religious Figures/Institutions \\
\hline Idlib Tawhid Brigade & Religious & 2012 & - & Syria & Bombing/Explosion & Government (General), Military \\
\hline Iduwini Volunteer Force (IVF) & Nationalist & 2006 & - & Niger & Armed Assault, Hostage Taking & Utilities \\
\hline Iduwini Youths & Nationalist & 2004 & - & Nigeria & Hostage Taking & Utilities \\
\hline Ikhwan Jammu and Kashmir & Nationalist & 1994 & 1994 & India & Hostage Taking, Unarmed Assault & $\begin{array}{l}\text { Government (General), Journalists \& } \\
\text { Media }\end{array}$ \\
\hline $\begin{array}{l}\text { Ikrima aka Abdikadir Mohamed } \\
\text { Abdikadir }\end{array}$ & Religious & 2013 & - & Somalia & Armed Assault, Bombing/Explosion & Unknown \\
\hline Illuminating Paths of Solidarity & Left-Wing & 2009 & 2009 & Greece & $\begin{array}{l}\text { Bombing/Explosion, } \\
\text { Facility/Infrastructure Attack }\end{array}$ & Government (General) \\
\hline Imam Bukhari Jamaat & Religious & 2013 & - & Syria, Tajikistan, Uzbekistan & Armed Assault & Military, Terrorists/Non-State Militia \\
\hline Imam Hussein Brigade & Nationalist & 2005 & 2005 & Iraq & Armed Assault, Bombing/Explosion & $\begin{array}{l}\text { Businesses, Government (Diplomatic), } \\
\text { Government (General) }\end{array}$ \\
\hline Imbonerakure & Nationalist & 2010 & - & Burundi & Armed Assault, Unarmed Assault & Violent Political Party \\
\hline Immediate Action & Left-Wing & 2003 & 2003 & Greece & Bombing/Explosion & Businesses \\
\hline Indian Mujahideen & Religious & 2007 & - & Bangladesh, India, Pakistan & Bombing/Explosion & $\begin{array}{l}\text { Businesses, Government (General), } \\
\text { Police, Private Citizens \& Property, } \\
\text { Religious Figures/Institutions, } \\
\text { Telecommunication, Transportation, } \\
\text { Violent Political Party }\end{array}$ \\
\hline $\begin{array}{l}\text { Indigenous Defense Front for the } \\
\text { Pastaza Province (FDIP) }\end{array}$ & Nationalist & 1998 & 1998 & Ecuador & Hostage Taking & Private Citizens \& Property, Utilities \\
\hline $\begin{array}{l}\text { Indigenous People's Federal Army } \\
\text { (IPFA)/Indigenous Federal State Army } \\
\text { (IFSA) }\end{array}$ & Nationalist & 2001 & 2001 & Philippines & Bombing/Explosion & $\begin{array}{l}\text { Journalists \& Media, } \\
\text { Telecommunication }\end{array}$ \\
\hline $\begin{array}{l}\text { Indigenous People's Front of Tripura } \\
\text { (IPFT) }\end{array}$ & Nationalist & 1997 & 2001 & India & Unknown & Unknown \\
\hline $\begin{array}{l}\text { Individualidades Tendiendo a lo Salvaje } \\
\text { (ITS) }\end{array}$ & Left-Wing & 2011 & - & Mexico & Armed Assault, Bombing/Explosion & $\begin{array}{l}\text { Educational Institutes, Private Citizens } \\
\text { \& Property }\end{array}$ \\
\hline
\end{tabular}




\begin{tabular}{|c|c|c|c|c|c|c|}
\hline VEO Name & Type & Emerged & Ended & Countries & Type of Attack & Target Type \\
\hline Indomitable Marxists & Left-Wing & 2003 & 2003 & Greece & Bombing/Explosion & Private Citizens \& Property \\
\hline Inevitables & Left-Wing & 2003 & 2003 & Bolivia & Bombing/Explosion & Educational Institutes, NGO \\
\hline Informal Anarchist Federation (FAI) & Left-Wing & 2003 & - & $\begin{array}{l}\text { Chile, Germany, Greece, Indonesia, } \\
\text { Italy, Mexico, Netherlands, Peru, Russia, } \\
\text { Spain, Switzerland, United Kingdom }\end{array}$ & $\begin{array}{l}\text { Assassination, Bombing/Explosion, } \\
\text { Facility/Infrastructure Attack }\end{array}$ & $\begin{array}{l}\text { Businesses, Educational Institutes, } \\
\text { Government (Diplomatic), Government } \\
\text { (General), Journalists \& Media, Military, } \\
\text { NGO, Police, Telecommunication, } \\
\text { Transportation, Violent Political Party }\end{array}$ \\
\hline Ingush Jama'at Shariat & Nationalist & 2006 & - & Russia & $\begin{array}{l}\text { Armed Assault, Assassination, } \\
\text { Bombing/Explosion, Hostage Taking }\end{array}$ & $\begin{array}{l}\text { Government (General), Private Citizens } \\
\text { \& Property }\end{array}$ \\
\hline International Justice Group & Religious & 1995 & 1995 & Egypt, Switzerland & Assassination & Government (Diplomatic) \\
\hline International Revolutionary Struggle & Left-Wing & 1997 & 1997 & Greece & Bombing/Explosion & $\begin{array}{l}\text { Airports \& Aircraft, Government } \\
\text { (General) }\end{array}$ \\
\hline Intifada Martyrs & Religious & 2000 & 2000 & Palestinian State & Assassination & Religious Figures/Institutions \\
\hline $\begin{array}{l}\text { Iraqi Islamic Vanguards for National } \\
\text { Salvation (IIVNS) }\end{array}$ & Religious & 1994 & 1999 & Iraq & Bombing/Explosion & $\begin{array}{l}\text { Journalists \& Media, Private Citizens \& } \\
\text { Property }\end{array}$ \\
\hline Iraqi Legitimate Resistance & Nationalist & 2004 & 2004 & Iraq & Hostage Taking & Private Citizens \& Property \\
\hline Iraqi National Congress (INC) & Nationalist & 1994 & 1994 & Iraq & Bombing/Explosion & Businesses, Police \\
\hline Iraqi Revenge Brigades & Nationalist & 2005 & 2005 & Iraq & Hostage Taking & $\begin{array}{l}\text { Government (General), Journalists \& } \\
\text { Media }\end{array}$ \\
\hline Iraq's Jihadist Leagues & Religious & 2008 & - & Iraq & Bombing/Explosion & Military, Private Citizens \& Property \\
\hline Irrintzi & Nationalist & 2006 & 2006 & France, Spain & Bombing/Explosion & Government (General), Military \\
\hline Islam Liberation Front & Religious & 1992 & 1994 & Algeria & Armed Assault, Assassination & Military, Police, Violent Political Party \\
\hline Islam Mujahiddin & Nationalist & 2012 & - & Pakistan & Armed Assault, Assassination & Violent Political Party \\
\hline Islami Inqilabi Mahaz & Religious & 1997 & - & India, Pakistan & Bombing/Explosion & $\begin{array}{l}\text { Businesses, Private Citizens \& Property, } \\
\text { Utilities }\end{array}$ \\
\hline Islami Jamiat-e-Talaba (IJT) & Nationalist & 2012 & - & Bangladesh, Pakistan & Armed Assault & Transportation \\
\hline Islamic al-Waqqas Brigade & Nationalist & 2004 & 2004 & Iraq & Hostage Taking & Government (General) \\
\hline Islamic Arab Front of Azawad (FIAA) & Nationalist & 1994 & 1994 & Mali & Armed Assault, Bombing/Explosion & Private Citizens \& Property \\
\hline Islamic Army in Iraq (IAI) & Religious & 2003 & - & Iraq & $\begin{array}{l}\text { Armed Assault, Assassination, } \\
\text { Bombing/Explosion, Hostage Taking }\end{array}$ & $\begin{array}{l}\text { Businesses, Government (Diplomatic), } \\
\text { Journalists \& Media, Military, Police, } \\
\text { Private Citizens \& Property }\end{array}$ \\
\hline Islamic Army of Yemen & Nationalist & 1998 & 2003 & Yemen & Bombing/Explosion, Hostage Taking & $\begin{array}{l}\text { Government (Diplomatic), NGO, } \\
\text { Tourists }\end{array}$ \\
\hline $\begin{array}{l}\text { Islamic Brigade of Shahids Riyad us- } \\
\text { Salikeheen/Black Widow Brigade }\end{array}$ & Nationalist & 2000 & - & Russia & $\begin{array}{l}\text { Armed Assault, Assassination, } \\
\text { Bombing/Explosion, Hostage Taking }\end{array}$ & $\begin{array}{l}\text { Airports \& Aircraft, Businesses, } \\
\text { Educational Institutes, Government } \\
\text { (General), Military, NGO, Tourists, } \\
\text { Transportation }\end{array}$ \\
\hline
\end{tabular}




\begin{tabular}{|c|c|c|c|c|c|c|}
\hline VEO Name & Type & Emerged & Ended & Countries & Type of Attack & Target Type \\
\hline Islamic Caliphate Army & Religious & 2013 & - & Russia, Syria & Armed Assault & Military \\
\hline Islamic Courts Union (ICU) & Religious & 2004 & - & Ethiopia, Somalia & $\begin{array}{l}\text { Armed Assault, Facility/Infrastructure } \\
\text { Attack, Hostage Taking }\end{array}$ & $\begin{array}{l}\text { Businesses, Government (Diplomatic), } \\
\text { Government (General), Military, NGO, } \\
\text { Private Citizens \& Property }\end{array}$ \\
\hline Islamic Defense Force & Religious & 1997 & 1998 & India & Bombing/Explosion & Transportation \\
\hline Islamic Fateh & Religious & 2003 & 2003 & India & Bombing/Explosion & Military \\
\hline Islamic Front - Jammu and Kashmir & Religious & 2002 & 2005 & India, Pakistan & $\begin{array}{l}\text { Armed Assault, Assassination, } \\
\text { Bombing/Explosion }\end{array}$ & $\begin{array}{l}\text { Government (General), Police, Religious } \\
\text { Figures/Institutions }\end{array}$ \\
\hline Islamic Front - Syria & Religious & 2013 & - & Syria & $\begin{array}{l}\text { Armed Assault, Bombing/Explosion, } \\
\text { Hostage Taking }\end{array}$ & $\begin{array}{l}\text { Government (Diplomatic), Government } \\
\text { (General), Journalists \& Media, Military, } \\
\text { Police, Private Citizens \& Property }\end{array}$ \\
\hline $\begin{array}{l}\text { Islamic Front for Iraqi Resistance } \\
\text { (JAMI) }\end{array}$ & Nationalist & 2005 & - & Iraq & Bombing/Explosion & Military, Religious Figures/Institutions \\
\hline $\begin{array}{l}\text { Islamic Glory Brigades in the Land of } \\
\text { the Nile (IGBLN) }\end{array}$ & Religious & 2005 & 2005 & Egypt & Bombing/Explosion & $\begin{array}{l}\text { Private Citizens \& Property, Religious } \\
\text { Figures/Institutions }\end{array}$ \\
\hline Islamic Golden Army & Religious & 1992 & 1992 & Germany & Armed Assault & Airports \& Aircraft \\
\hline $\begin{array}{l}\text { Islamic International Peacekeeping } \\
\text { Brigade (IIPB) }\end{array}$ & Religious & 1998 & 2002 & Azerbaijan, Georgia, Russia, Turkey & $\begin{array}{l}\text { Armed Assault, Assassination, } \\
\text { Bombing/Explosion }\end{array}$ & Military, Private Citizens \& Property \\
\hline Islamic Jihad Brigades & Religious & 2004 & - & Iraq & Assassination, Hostage Taking & $\begin{array}{l}\text { Government (General), Military, Private } \\
\text { Citizens \& Property }\end{array}$ \\
\hline Islamic Jihad Front & Nationalist & 2002 & - & India & Assassination & Government (General) \\
\hline Islamic Jihad Group (IJG) & Religious & 2004 & 2004 & Uzbekistan & Bombing/Explosion & $\begin{array}{l}\text { Government (Diplomatic), Government } \\
\text { (General) }\end{array}$ \\
\hline Islamic Jihad Union (IJU) & Nationalist & 2002 & - & $\begin{array}{l}\text { Afghanistan, Kazakhstan, Kyrgyzstan, } \\
\text { Pakistan, Uzbekistan }\end{array}$ & Armed Assault, Bombing/Explosion & $\begin{array}{l}\text { Businesses, Government (Diplomatic), } \\
\text { Police, Private Citizens \& Property }\end{array}$ \\
\hline $\begin{array}{l}\text { Islamic League for Da'wa and Jihad } \\
\text { (LIDD) }\end{array}$ & Nationalist & 1997 & - & Algeria & Unknown & Unknown \\
\hline Islamic Movement of Azawad (IMA) & Religious & 2013 & - & Mali & Armed Assault, Bombing/Explosion & Terrorists/Non-State Militia \\
\hline Islamic Movement of Holy Warriors & Nationalist & 2004 & - & Iraq & Hostage Taking & Government (General), Military \\
\hline Islamic Movement of Iraq & Religious & 2014 & - & Iran, Iraq & Armed Assault & Terrorists/Non-State Militia \\
\hline Islamic Movement of Kyrgyzstan (IMK) & Religious & 2011 & - & Kyrgyzstan & Armed Assault & Government (General) \\
\hline Islamic Movement of Uzbekistan (IMU) & Religious & 2000 & 2015 & $\begin{array}{l}\text { Afghanistan, Kyrgyzstan, Tajikistan, } \\
\text { Uzbekistan }\end{array}$ & Bombing/Explosion, Hostage Taking & $\begin{array}{l}\text { Police, Private Citizens \& Property, } \\
\text { Tourists }\end{array}$ \\
\hline Islamic Party of Kenya & Religious & 1992 & 1992 & Kenya & Armed Assault & Police, Violent Political Party \\
\hline Islamic Rage Brigade & Religious & 2004 & - & Iraq & Armed Assault, Hostage Taking & Government (General), Military \\
\hline Islamic Resistance Brigades & Religious & 2004 & - & Iraq, Lebanon & Bombing/Explosion, Hostage Taking & $\begin{array}{l}\text { Businesses, Government (General), } \\
\text { Military, Police, Violent Political Party }\end{array}$ \\
\hline
\end{tabular}




\begin{tabular}{|c|c|c|c|c|c|c|}
\hline VEO Name & Type & Emerged & Ended & Countries & Type of Attack & Target Type \\
\hline $\begin{array}{l}\text { Islamic Resistance in Iraq-The Truthful } \\
\text { Promise Corps (FWS) }\end{array}$ & Religious & 2003 & - & Iran, Iraq, Syria & Armed Assault & Terrorists/Non-State Militia \\
\hline Islamic Revival Movement & Religious & 1995 & 1995 & Algeria, Tajikistan & Assassination & Military \\
\hline Islamic Revolution to Liberate Palestine & Religious & 2000 & 2000 & Israel & Bombing/Explosion & Transportation \\
\hline Islamic Revolutionary Front & Religious & 2005 & 2005 & India & Bombing/Explosion & $\begin{array}{l}\text { Businesses, Private Citizens \& Property, } \\
\text { Utilities }\end{array}$ \\
\hline Islamic Shashantantra Andolon (ISA) & Religious & 2002 & 2004 & Bangladesh & Bombing/Explosion & $\begin{array}{l}\text { Businesses, Government (General), } \\
\text { Private Citizens \& Property }\end{array}$ \\
\hline $\begin{array}{l}\text { Islamic State (IS)/Islamic State of Iraq } \\
\text { and ash-Sham (ISIS)/Islamic State of } \\
\text { Iraq and the Levant (ISIS, ISIL, IS) }\end{array}$ & Religious & 2013 & - & $\begin{array}{l}\text { Indonesia, Iraq, Libya, Malaysia, } \\
\text { Nigeria, Palestinian State, Saudi Arabia, } \\
\text { Syria, United States, Yemen }\end{array}$ & $\begin{array}{l}\text { Armed Assault, Assassination, } \\
\text { Bombing/Explosion, } \\
\text { Facility/Infrastructure Attack, Hostage } \\
\text { Taking }\end{array}$ & $\begin{array}{l}\text { Businesses, Educational Institutes, Food } \\
\text { or Water Supply, Government (General), } \\
\text { Journalists \& Media, Military, NGO, } \\
\text { Police, Private Citizens \& Property, } \\
\text { Religious Figures/Institutions, } \\
\text { Terrorists/Non-State Militia, Tourists, } \\
\text { Transportation, Utilities, Violent } \\
\text { Political Party }\end{array}$ \\
\hline Islamic State Algeria (IS, ISA, ISISA) & Religious & 2014 & - & Algeria, Tunisia & Armed Assault, Bombing/Explosion & Military, Police \\
\hline Islamic State Bangladesh (ISB, ISISB) & Religious & 2014 & - & Bangladesh & $\begin{array}{l}\text { Armed Assault, Assassination, } \\
\text { Bombing/Explosion, Hostage Taking }\end{array}$ & $\begin{array}{l}\text { Businesses, Educational Institutes, } \\
\text { NGO, Police, Private Citizens \& } \\
\text { Property, Religious Figures/Institutions, } \\
\text { Violent Political Party }\end{array}$ \\
\hline Islamic State Brazil & Religious & 2009 & - & Brazil, Egypt, Lebanon, Syria & Armed Assault & Unknown \\
\hline Islamic State in Greater Sahara (ISGS) & Religious & 2015 & - & Burkina Faso, Mali, Morocco, Niger & $\begin{array}{l}\text { Armed Assault, Bombing/Explosion, } \\
\text { Hostage Taking }\end{array}$ & $\begin{array}{l}\text { Military, Police, Private Citizens \& } \\
\text { Property }\end{array}$ \\
\hline Islamic State in Libya (ISL, ISISL) & Religious & 2014 & - & Libya, Tunisia & $\begin{array}{l}\text { Armed Assault, Assassination, } \\
\text { Bombing/Explosion, } \\
\text { Facility/Infrastructure Attack, Hostage } \\
\text { Taking }\end{array}$ & $\begin{array}{l}\text { Airports \& Aircraft, Businesses, } \\
\text { Educational Institutes, Government } \\
\text { (Diplomatic), Government (General), } \\
\text { Journalists \& Media, Military, Police, } \\
\text { Private Citizens \& Property, Religious } \\
\text { Figures/Institutions, Terrorists/Non- } \\
\text { State Militia, Utilities }\end{array}$ \\
\hline $\begin{array}{l}\text { Islamic State in Saudi Arabia (ISKSA, } \\
\text { ISISKSA) }\end{array}$ & Religious & 2015 & - & Saudi Arabia & $\begin{array}{l}\text { Armed Assault, Assassination, } \\
\text { Bombing/Explosion }\end{array}$ & $\begin{array}{l}\text { Businesses, Government (Diplomatic), } \\
\text { Government (General), Police, Private } \\
\text { Citizens \& Property, Religious } \\
\text { Figures/Institutions, Terrorists/Non- } \\
\text { State Militia }\end{array}$ \\
\hline
\end{tabular}




\begin{tabular}{|c|c|c|c|c|c|c|}
\hline VEO Name & Type & Emerged & Ended & Countries & Type of Attack & Target Type \\
\hline Islamic State in Somalia & Religious & 2015 & - & Somalia & Armed Assault & $\begin{array}{l}\text { Military, Police, Terrorists/Non-State } \\
\text { Militia }\end{array}$ \\
\hline Islamic State in Yemen (ISY, ISISY) & Religious & 2015 & - & Yemen & $\begin{array}{l}\text { Assassination, Bombing/Explosion, } \\
\text { Hostage Taking }\end{array}$ & $\begin{array}{l}\text { Government (General), Journalists \& } \\
\text { Media, Military, Police, Private Citizens } \\
\text { \& Property, Religious } \\
\text { Figures/Institutions, Terrorists/Non- } \\
\text { State Militia, Violent Political Party }\end{array}$ \\
\hline Islamic State Khurasan (IS, ISK, ISISK) & Religious & 2014 & - & Afghanistan, Pakistan, Uzbekistan & Armed Assault, Bombing/Explosion & Military, Police \\
\hline $\begin{array}{l}\text { Islamic State Malaysia, Indonesia \& } \\
\text { Philippines (IS, ISMIP, ISISMIP, } \\
\text { JAKDN) }\end{array}$ & Religious & 2014 & - & $\begin{array}{l}\text { Indonesia, Iraq, Malaysia, Philippines, } \\
\text { Syria }\end{array}$ & Armed Assault, Bombing/Explosion & $\begin{array}{l}\text { Government (General), Police, } \\
\text { Terrorists/Non-State Militia }\end{array}$ \\
\hline Islamic State Maldives (ISM, ISISM) & Religious & 2014 & - & Maldives, Syria & Armed Assault & $\begin{array}{l}\text { Government (General), Military, Police, } \\
\text { Terrorists/Non-State Militia }\end{array}$ \\
\hline Islamic State of India (ISI/ISISI) & Religious & 2015 & - & India, Syria & Armed Assault, Bombing/Explosion & $\begin{array}{l}\text { Government (General), Military, Private } \\
\text { Citizens \& Property }\end{array}$ \\
\hline Islamic State of Iraq (ISI) & Religious & 2007 & - & Iraq & $\begin{array}{l}\text { Armed Assault, Assassination, } \\
\text { Bombing/Explosion, } \\
\text { Facility/Infrastructure Attack, Hostage } \\
\text { Taking }\end{array}$ & $\begin{array}{l}\text { Businesses, Educational Institutes, } \\
\text { Government (Diplomatic), Government } \\
\text { (General), Journalists \& Media, Military, } \\
\text { Police, Private Citizens \& Property, } \\
\text { Terrorists/Non-State Militia, } \\
\text { Transportation }\end{array}$ \\
\hline $\begin{array}{l}\text { Islamic State Senegalese Foreign Fighter } \\
\text { Units (ISSFF, ISISSFF) }\end{array}$ & Religious & 2015 & - & Libya, Senegal, Syria & Armed Assault, Bombing/Explosion & Terrorists/Non-State Militia \\
\hline Islamic State Sinai (IS, ISS, ISISS) & Religious & 2014 & - & Egypt, Palestinian State & $\begin{array}{l}\text { Armed Assault, Assassination, } \\
\text { Bombing/Explosion, } \\
\text { Facility/Infrastructure Attack, } \\
\text { Hijacking, Hostage Taking }\end{array}$ & $\begin{array}{l}\text { Abortion Related, Businesses, } \\
\text { Educational Institutes, Government } \\
\text { (Diplomatic), Government (General), } \\
\text { Military, Police, Private Citizens \& } \\
\text { Property, Religious Figures/Institutions, } \\
\text { Terrorists/Non-State Militia, Tourists, } \\
\text { Transportation, Utilities }\end{array}$ \\
\hline $\begin{array}{l}\text { Islamic State West Africa (ISWA, } \\
\text { ISWAP) }\end{array}$ & Religious & 2016 & - & Cameroon, Chad, Niger, Nigeria & Armed Assault, Bombing/Explosion & Military \\
\hline $\begin{array}{l}\text { Islamic Swords of Justice in the Land of } \\
\text { Ribat }\end{array}$ & Religious & 2006 & 2006 & Israel, Palestinian State & Bombing/Explosion & Businesses \\
\hline Islamic Warriors & Religious & 1993 & 1993 & Bosnia and Herzegovina & Armed Assault & Private Citizens \& Property \\
\hline Islamic Youth Shura Council & Religious & 2014 & - & Libya & $\begin{array}{l}\text { Bombing/Explosion, Hostage Taking, } \\
\text { Unarmed Assault }\end{array}$ & Private Citizens \& Property \\
\hline Istabu Freedom Movement & Nationalist & 1999 & 2000 & Solomon Islands & Armed Assault & Airports \& Aircraft \\
\hline
\end{tabular}




\begin{tabular}{|c|c|c|c|c|c|c|}
\hline VEO Name & Type & Emerged & Ended & Countries & $\begin{array}{c}\text { Type of Attack } \\
\end{array}$ & Target Type \\
\hline Italian al-Shabaab & Nationalist & 2012 & - & Somalia & Armed Assault, Bombing/Explosion & Police \\
\hline Ivorian Popular Front (FPI) & Left-Wing & 1992 & 2007 & Cote D'Ivoire & Hostage Taking, Unarmed Assault & Government (General) \\
\hline Izz ad-Din al-Qassam Brigades & Religious & 1992 & - & Palestinian State & Armed Assault & Military, Private Citizens \& Property \\
\hline Jabhat al-Islamiyaa as-Suriya & Nationalist & 2012 & - & Syria & Armed Assault & Government (General), Military \\
\hline Jabhat an-Nusra li-Ahl Misr & Religious & 2013 & - & Egypt & Armed Assault & $\begin{array}{l}\text { Terrorists/Non-State Militia, Violent } \\
\text { Political Party }\end{array}$ \\
\hline Jabhat Ansar ud-Din & Religious & 2014 & - & Russia, Syria & Armed Assault, Bombing/Explosion & Government (General) \\
\hline Jabhat Fateh al-Sham (JFS) & Religious & 2011 & - & Lebanon, Russia, Syria & $\begin{array}{l}\text { Armed Assault, Assassination, } \\
\text { Bombing/Explosion }\end{array}$ & $\begin{array}{l}\text { Government (General), Military, } \\
\text { Religious Figures/Institutions, } \\
\text { Terrorists/Non-State Militia, Violent } \\
\text { Political Party }\end{array}$ \\
\hline Jadid al-Qa'idah Bangladesh (JAQB) & Religious & 2007 & - & Bangladesh & Bombing/Explosion & Educational Institutes, Transportation \\
\hline $\begin{array}{l}\text { Jagrata Muslim Janata Bangladesh } \\
\text { (JMJB) }\end{array}$ & Religious & 1998 & - & Bangladesh & Armed Assault, Bombing/Explosion & Private Citizens \& Property \\
\hline Jagrata Towhidi Janata & Religious & 1996 & 1996 & Bangladesh & Bombing/Explosion & Journalists \& Media \\
\hline $\begin{array}{l}\text { Jahba East Africa/ISIS Somalia, Kenya, } \\
\text { Tanzania and Uganda/ISISSKTU }\end{array}$ & Religious & 2016 & - & Kenya, Somalia, Tanzania, Uganda & Armed Assault, Bombing/Explosion & $\begin{array}{l}\text { Government (General), Military, } \\
\text { Religious Figures/Institutions, } \\
\text { Terrorists/Non-State Militia }\end{array}$ \\
\hline Jaish al Mu'minun & Religious & 2010 & - & Palestinian State & Armed Assault & $\begin{array}{l}\text { Government (General), Religious } \\
\text { Figures/Institutions }\end{array}$ \\
\hline Jaish al-Adl (Army of Justice) & Religious & 2012 & - & Iran, Pakistan & $\begin{array}{l}\text { Armed Assault, Bombing/Explosion, } \\
\text { Hostage Taking }\end{array}$ & Government (General), Military, Police \\
\hline Jaish al-Ta'ifa al-Mansoura & Religious & 2003 & 2005 & Iraq & Bombing/Explosion, Hostage Taking & $\begin{array}{l}\text { Businesses, Military, Religious } \\
\text { Figures/Institutions }\end{array}$ \\
\hline Jaish al-Tawhid & Religious & 2012 & - & Syria & Armed Assault & Military \\
\hline $\begin{array}{l}\text { Jaish al-Ummah (JAU)/Army of the } \\
\text { Nation }\end{array}$ & Religious & 2008 & - & Israel, Palestinian State & Armed Assault, Bombing/Explosion & $\begin{array}{l}\text { Government (General), Private Citizens } \\
\text { \& Property }\end{array}$ \\
\hline Jaish-e-Islam & Nationalist & 2008 & & Pakistan & $\begin{array}{l}\text { Armed Assault, Assassination, } \\
\text { Bombing/Explosion }\end{array}$ & $\begin{array}{l}\text { Police, Private Citizens \& Property, } \\
\text { Transportation }\end{array}$ \\
\hline Jaish-e-Mohammad (JeM) & Nationalist & 2000 & - & Afghanistan, India, Pakistan & $\begin{array}{l}\text { Armed Assault, Assassination, } \\
\text { Bombing/Explosion, Hostage Taking }\end{array}$ & $\begin{array}{l}\text { Educational Institutes, Government } \\
\text { (Diplomatic), Government (General), } \\
\text { Journalists \& Media, Military, Police, } \\
\text { Private Citizens \& Property, Religious } \\
\text { Figures/Institutions, Transportation }\end{array}$ \\
\hline Jaljalat & Nationalist & 2009 & - & Israel, Palestinian State & $\begin{array}{l}\text { Armed Assault, Bombing/Explosion, } \\
\text { Hostage Taking }\end{array}$ & Private Citizens \& Property \\
\hline
\end{tabular}




\begin{tabular}{|c|c|c|c|c|c|c|}
\hline VEO Name & Type & Emerged & Ended & Countries & Type of Attack & Target Type \\
\hline $\begin{array}{l}\text { Jama'a a-Islamiya lil-Tawhid wal-Jihad } \\
\text { (JITJ) }\end{array}$ & Religious & 2011 & - & Algeria & Assassination, Bombing/Explosion & $\begin{array}{l}\text { Journalists \& Media, Private Citizens \& } \\
\text { Property }\end{array}$ \\
\hline Jama'a al-Islamiya al-Alamiya (JIA) & Religious & 2004 & - & Egypt & Bombing/Explosion & Businesses, Military \\
\hline Jamaah Ansharusy Syariah (JAS) & Religious & 2014 & - & Indonesia & Unknown & Police, Terrorists/Non-State Militia \\
\hline Jama'ah Anshorut Tauhid (JAT) & Religious & 2008 & - & Indonesia, Singapore & $\begin{array}{l}\text { Armed Assault, Assassination, } \\
\text { Bombing/Explosion, Unarmed Assault }\end{array}$ & $\begin{array}{l}\text { Government (Diplomatic), Government } \\
\text { (General), NGO, Police, Religious } \\
\text { Figures/Institutions }\end{array}$ \\
\hline Jama'at Ahadun Ahad & Religious & 2014 & - & Russia, Syria, Turkey & Armed Assault & Government (General) \\
\hline $\begin{array}{l}\text { Jama'at al-Ja'wat al-Quran wal Sonat } \\
\text { (JJQS) }\end{array}$ & Religious & 2007 & - & Afghanistan, Pakistan & $\begin{array}{l}\text { Armed Assault, Bombing/Explosion, } \\
\text { Hostage Taking }\end{array}$ & $\begin{array}{l}\text { Military, NGO, Private Citizens \& } \\
\text { Property }\end{array}$ \\
\hline Jama'at al-Muvahidun & Religious & 2002 & - & Azerbaijan & $\begin{array}{l}\text { Armed Assault, Assassination, } \\
\text { Bombing/Explosion }\end{array}$ & $\begin{array}{l}\text { Businesses, Government (Diplomatic), } \\
\text { NGO, Police, Private Citizens \& Property }\end{array}$ \\
\hline $\begin{array}{l}\text { Jama'at Ansar al-Furqan in Bilad al- } \\
\text { Sham (JAFBS) }\end{array}$ & Religious & 2016 & - & Jordan, Syria & Unknown & Unknown \\
\hline Jama'at Ansar al-Sunnah & Religious & 2003 & - & Iraq & $\begin{array}{l}\text { Armed Assault, Bombing/Explosion, } \\
\text { Hostage Taking }\end{array}$ & $\begin{array}{l}\text { Airports \& Aircraft, Government } \\
\text { (Diplomatic), Government (General), } \\
\text { Military, Police }\end{array}$ \\
\hline Jama'at Ansarullah (JA) & Religious & 2010 & - & Tajikistan & Bombing/Explosion & Unknown \\
\hline Jama'at Jund al-Islam & Religious & 2013 & - & Egypt & Armed Assault & Military, Terrorists/Non-State Militia \\
\hline $\begin{array}{l}\text { Jama'atu Ansarul Muslimina Fi Biladis } \\
\text { Sudan (JAMBS-Ansaru) }\end{array}$ & Religious & 2012 & - & $\begin{array}{l}\text { Cameroon, Mali, Mauritania, Nigeria, } \\
\text { Sudan }\end{array}$ & $\begin{array}{l}\text { Armed Assault, Bombing/Explosion, } \\
\text { Facility/Infrastructure Attack, Hostage } \\
\text { Taking }\end{array}$ & $\begin{array}{l}\text { Businesses, Military, NGO, Police, } \\
\text { Private Citizens \& Property }\end{array}$ \\
\hline Jama'atul Mujahidin Bangladesh (JMB) & Religious & 1998 & - & Bangladesh & $\begin{array}{l}\text { Armed Assault, Assassination, } \\
\text { Bombing/Explosion, } \\
\text { Facility/Infrastructure Attack }\end{array}$ & $\begin{array}{l}\text { Businesses, Government (General), } \\
\text { Journalists \& Media, Police, Private } \\
\text { Citizens \& Property, Religious } \\
\text { Figures/Institutions }\end{array}$ \\
\hline Jama'atul-Ahrar (TTPJA) & Religious & 2015 & - & Pakistan & $\begin{array}{l}\text { Armed Assault, Assassination, } \\
\text { Bombing/Explosion, Hostage Taking }\end{array}$ & $\begin{array}{l}\text { Government (General), Military, Police, } \\
\text { Private Citizens \& Property, Religious } \\
\text { Figures/Institutions, Terrorists/Non- } \\
\text { State Militia, Violent Political Party }\end{array}$ \\
\hline Jamal Network (MJN) & Religious & 2000 & - & Egypt, Libya, Yemen & Bombing/Explosion & Government (Diplomatic), Maritime \\
\hline $\begin{array}{l}\text { Jamiat al-Islah al-Idzhtimal - Social } \\
\text { Reform }\end{array}$ & Nationalist & 2003 & - & Russia & Unknown & Government (General), Military \\
\hline Jamiat-e Islami-yi Afghanistan & Religious & 1992 & 1994 & Afghanistan & $\begin{array}{l}\text { Armed Assault, Assassination, Hostage } \\
\text { Taking }\end{array}$ & $\begin{array}{l}\text { Government (General), Journalists \& } \\
\text { Media, Violent Political Party }\end{array}$ \\
\hline Jamiyyatul Salaf (JS) & Religious & 2011 & - & Maldives & Armed Assault, Unarmed Assault & Private Citizens \& Property \\
\hline
\end{tabular}




\begin{tabular}{|c|c|c|c|c|c|c|}
\hline VEO Name & Type & Emerged & Ended & Countries & Type of Attack & Target Type \\
\hline Jammu and Kashmir Freedom Force & Nationalist & 2003 & - & India, Pakistan & Armed Assault, Bombing/Explosion & $\begin{array}{l}\text { Businesses, Government (General), } \\
\text { Police, Telecommunication }\end{array}$ \\
\hline Jammu and Kashmir Islamic Front & Nationalist & 1994 & - & India, Pakistan & Armed Assault, Bombing/Explosion & $\begin{array}{l}\text { Businesses, Police, Private Citizens \& } \\
\text { Property, Transportation }\end{array}$ \\
\hline $\begin{array}{l}\text { Janatantrik Tarai Madhes Mukti Party } \\
\text { (JTMM-P) }\end{array}$ & Nationalist & 2007 & - & Nepal & Bombing/Explosion & Private Citizens \& Property \\
\hline $\begin{array}{l}\text { Janatantrik Terai Madhesh Mukti } \\
\text { Morcha (JTMM-M)/Terai Madheshi } \\
\text { Mukti Morcha (TMMM) }\end{array}$ & Nationalist & 2010 & - & Nepal & Armed Assault, Bombing/Explosion & $\begin{array}{l}\text { Businesses, Educational Institutes, } \\
\text { Government (General), Military, Police, } \\
\text { Violent Political Party }\end{array}$ \\
\hline $\begin{array}{l}\text { Janatantrik Terai Mukti Morcha - } \\
\text { Bisphot Singh (JTMM-B) }\end{array}$ & Nationalist & 2006 & 2007 & Nepal & $\begin{array}{l}\text { Bombing/Explosion, } \\
\text { Facility/Infrastructure Attack, Hostage } \\
\text { Taking }\end{array}$ & $\begin{array}{l}\text { Government (General), Private Citizens } \\
\text { \& Property }\end{array}$ \\
\hline $\begin{array}{l}\text { Janatantrik Terai Mukti Morcha - Goit } \\
\text { (JTMM-G) }\end{array}$ & Nationalist & 2006 & - & Nepal & $\begin{array}{l}\text { Armed Assault, Assassination, } \\
\text { Bombing/Explosion, Hostage Taking }\end{array}$ & $\begin{array}{l}\text { Businesses, Educational Institutes, } \\
\text { Government (General), Police, Private } \\
\text { Citizens \& Property, Transportation, } \\
\text { Violent Political Party }\end{array}$ \\
\hline $\begin{array}{l}\text { Janatantrik Terai Mukti Morcha - Jwala } \\
\text { Singh (JTMM-J) }\end{array}$ & Nationalist & 2006 & - & Nepal & $\begin{array}{l}\text { Armed Assault, Bombing/Explosion, } \\
\text { Facility/Infrastructure Attack, Hostage } \\
\text { Taking }\end{array}$ & $\begin{array}{l}\text { Businesses, Educational Institutes, } \\
\text { Government (General), Journalists \& } \\
\text { Media, NGO, Police, Private Citizens \& } \\
\text { Property, Telecommunication, } \\
\text { Transportation, Violent Political Party }\end{array}$ \\
\hline $\begin{array}{l}\text { Janatantrik Terai Mukti Morcha - Rajan } \\
\text { Mukti (JTMM-R) }\end{array}$ & Nationalist & 2008 & - & Nepal & $\begin{array}{l}\text { Armed Assault, Assassination, } \\
\text { Bombing/Explosion, Hostage Taking }\end{array}$ & $\begin{array}{l}\text { Government (General), Journalists \& } \\
\text { Media, NGO, Police, Private Citizens \& } \\
\text { Property, Violent Political Party }\end{array}$ \\
\hline $\begin{array}{l}\text { Janatantrik Terai Mukti Morcha } \\
\text { (JTMM)/Akhil Terai Mukti Morcha } \\
\text { (ATMM) }\end{array}$ & Nationalist & 2006 & - & India, Nepal & $\begin{array}{l}\text { Armed Assault, Bombing/Explosion, } \\
\text { Hostage Taking }\end{array}$ & $\begin{array}{l}\text { Businesses, Government (General), } \\
\text { Police, Private Citizens \& Property }\end{array}$ \\
\hline Jaysh al-Fateh/Army of Conquest & Nationalist & 2015 & - & Syria & Armed Assault, Bombing/Explosion & $\begin{array}{l}\text { Educational Institutes, Military, Private } \\
\text { Citizens \& Property }\end{array}$ \\
\hline Jaysh al-Islam/Brigades of Islam & Nationalist & 2012 & - & Lebanon, Syria & $\begin{array}{l}\text { Armed Assault, Assassination, } \\
\text { Bombing/Explosion, Hostage Taking }\end{array}$ & $\begin{array}{l}\text { Government (Diplomatic), Government } \\
\text { (General), Military, Police, Private } \\
\text { Citizens \& Property, Terrorists/Non- } \\
\text { State Militia }\end{array}$ \\
\hline Jaysh al-Izza wa-Karama (ADP) & Religious & 2013 & - & Iraq & Armed Assault, Bombing/Explosion & Government (General) \\
\hline Jaysh al-Mahdi & Religious & 2003 & - & Iraq & Armed Assault & Military \\
\hline Jaysh al-Muslimin & Nationalist & 2004 & - & Afghanistan & $\begin{array}{l}\text { Armed Assault, Facility/Infrastructure } \\
\text { Attack, Hostage Taking }\end{array}$ & Government (General), NGO, Utilities \\
\hline
\end{tabular}




\begin{tabular}{|c|c|c|c|c|c|c|}
\hline VEO Name & Type & Emerged & Ended & Countries & Type of Attack & Target Type \\
\hline Jaysh al-Tariqa al-Naqshbandia (JRTN) & Nationalist & 2006 & - & Iraq & Armed Assault & $\begin{array}{l}\text { Government (General), Military, } \\
\text { Terrorists/Non-State Militia }\end{array}$ \\
\hline Jaysh Muhammad (JM) & Religious & 2004 & - & Iraq & $\begin{array}{l}\text { Armed Assault, Bombing/Explosion, } \\
\text { Facility/Infrastructure Attack }\end{array}$ & Military \\
\hline Jaysh Muhammad in Bilad al-Sham & Religious & 2013 & - & Egypt, Syria & Armed Assault & Government (General) \\
\hline $\begin{array}{l}\text { Jean-Marc Rouillan Armed and } \\
\text { Heartless Columns }\end{array}$ & Left-Wing & 2008 & - & Chile & Bombing/Explosion & Businesses \\
\hline Jemaah Islamiya (JI) & Religious & 1993 & - & Indonesia, Philippines & $\begin{array}{l}\text { Armed Assault, Assassination, } \\
\text { Bombing/Explosion }\end{array}$ & $\begin{array}{l}\text { Airports \& Aircraft, Businesses, } \\
\text { Government (Diplomatic), Government } \\
\text { (General), Military, Police, Private } \\
\text { Citizens \& Property, Religious } \\
\text { Figures/Institutions, Tourists, Utilities }\end{array}$ \\
\hline Jenin Martyr's Brigade & Nationalist & 2003 & - & Israel & Hostage Taking & Police \\
\hline Jerusalem Groups Hebrew & Religious & 2002 & 2002 & Israel & Bombing/Explosion & Businesses, Private Citizens \& Property \\
\hline Jeshullah & Religious & 1999 & 1999 & Azerbaijan, Russia & Bombing/Explosion & Businesses, Government (Diplomatic) \\
\hline Jewish Fighting Organization (EYAL) & Nationalist & 1993 & 1995 & Israel & Assassination & Government (General) \\
\hline Jharkhand Prastuti Committee (JPC) & Left-Wing & 2014 & - & India & $\begin{array}{l}\text { Facility/Infrastructure Attack, Hostage } \\
\text { Taking }\end{array}$ & Businesses, Private Citizens \& Property \\
\hline Jihad in Sweden & Religious & 2005 & 2005 & Sweden & Bombing/Explosion & Government (General) \\
\hline Jihad Islamic League Front & Religious & 1997 & 1997 & Algeria & Bombing/Explosion & Businesses \\
\hline Jihad Pegah & Nationalist & 2005 & - & Iraq & Bombing/Explosion & Government (General) \\
\hline Jihad Talaat al-Fatah & Religious & 1997 & 1998 & Egypt & $\begin{array}{l}\text { Armed Assault, Bombing/Explosion, } \\
\text { Hijacking }\end{array}$ & Tourists \\
\hline Jihad-e-Islam Pakistan & Religious & 2012 & - & France, Pakistan & $\begin{array}{l}\text { Armed Assault, Bombing/Explosion, } \\
\text { Hostage Taking }\end{array}$ & $\begin{array}{l}\text { Educational Institutes, Private Citizens } \\
\text { \& Property }\end{array}$ \\
\hline Joint Meeting Parties (JMP) & Nationalist & 2002 & - & Yemen & Bombing/Explosion & Government (General), Military \\
\hline $\begin{array}{l}\text { Joint Niger Delta Liberation Force } \\
\text { (JNDLF) }\end{array}$ & Nationalist & 2016 & - & Nigeria & Armed Assault, Bombing/Explosion & Government (General) \\
\hline Joint Revolutionary Council & Nationalist & 2006 & - & Nigeria & Armed Assault & Businesses, Utilities \\
\hline Joint Task Force (JTF) Nigeria & Nationalist & 2012 & - & Nigeria & Unknown & Terrorists/Non-State Militia \\
\hline Jordanian Islamic Resistance & Religious & 1997 & 2000 & Jordan & Armed Assault & Government (Diplomatic) \\
\hline Jordanian Salafi Jihadist Movement & Religious & 2009 & - & Jordan & Bombing/Explosion & NGO \\
\hline Juan Antonio Lavalleja Command & $\begin{array}{l}\text { Right- } \\
\text { Wing }\end{array}$ & 1992 & 1992 & Uruguay & Bombing/Explosion & Transportation \\
\hline July 2oth Brigade & Left-Wing & 2002 & 2002 & Italy & Armed Assault & Police \\
\hline Junaid Jihadist Battalion & Religious & 2005 & 2005 & Iraq & Hostage Taking & Government (General), Military \\
\hline
\end{tabular}




\begin{tabular}{|c|c|c|c|c|c|c|}
\hline VEO Name & Type & Emerged & Ended & Countries & Type of Attack & Target Type \\
\hline Jund al-Aqsa & Religious & 2012 & - & Syria & Armed Assault, Bombing/Explosion & $\begin{array}{l}\text { Government (General), Military, Private } \\
\text { Citizens \& Property }\end{array}$ \\
\hline $\begin{array}{l}\text { Jund al-Khilafah (JaK)/Army of the } \\
\text { Caliphate }\end{array}$ & Nationalist & 2011 & - & $\begin{array}{l}\text { Afghanistan, Egypt, Kazakhstan, } \\
\text { Pakistan, Tunisia }\end{array}$ & $\begin{array}{l}\text { Armed Assault, Bombing/Explosion, } \\
\text { Hostage Taking }\end{array}$ & $\begin{array}{l}\text { Businesses, Government (General), } \\
\text { Military, Private Citizens \& Property, } \\
\text { Tourists }\end{array}$ \\
\hline $\begin{array}{l}\text { Jund Allah Organization for the Sunni } \\
\text { Mujahideen in Iran }\end{array}$ & Religious & 2005 & - & Iran & $\begin{array}{l}\text { Armed Assault, Bombing/Explosion, } \\
\text { Hostage Taking }\end{array}$ & $\begin{array}{l}\text { Educational Institutes, Government } \\
\text { (Diplomatic), Government (General), } \\
\text { Military, Police, Private Citizens \& } \\
\text { Property }\end{array}$ \\
\hline Jund al-Qawqaz & Religious & 2013 & - & Russia, Syria & Armed Assault & Government (General) \\
\hline Jund al-Sham for Tawhid and Jihad & Religious & 1999 & - & $\begin{array}{l}\text { Afghanistan, Lebanon, Palestinian State, } \\
\text { Qatar, Syria }\end{array}$ & $\begin{array}{l}\text { Armed Assault, Assassination, } \\
\text { Bombing/Explosion }\end{array}$ & $\begin{array}{l}\text { Businesses, Government (Diplomatic), } \\
\text { Government (General), Military, NGO, } \\
\text { Police, Private Citizens \& Property, } \\
\text { Terrorists/Non-State Militia, Violent } \\
\text { Political Party }\end{array}$ \\
\hline Jundallah-Pakistan & Religious & 2004 & - & Afghanistan, Pakistan, Uzbekistan & $\begin{array}{l}\text { Assassination, Bombing/Explosion, } \\
\text { Hostage Taking }\end{array}$ & $\begin{array}{l}\text { Businesses, Government (General), } \\
\text { Military, NGO, Police, Private Citizens \& } \\
\text { Property, Religious Figures/Institutions, } \\
\text { Tourists, Transportation }\end{array}$ \\
\hline Jundul Hafsa & Religious & 2008 & - & Pakistan & Unknown & Religious Figures/Institutions, Tourists \\
\hline Just Punishment Brigades & Religious & 2007 & - & Iraq & Armed Assault, Bombing/Explosion & Police \\
\hline Justice and Equality Movement (JEM) & Religious & 2003 & - & Sudan & $\begin{array}{l}\text { Armed Assault, Bombing/Explosion, } \\
\text { Facility/Infrastructure Attack, Hostage } \\
\text { Taking }\end{array}$ & $\begin{array}{l}\text { Airports \& Aircraft, Businesses, } \\
\text { Government (Diplomatic), Government } \\
\text { (General), Military, Police, Private } \\
\text { Citizens \& Property }\end{array}$ \\
\hline Justice Army of Defenseless People & Left-Wing & 1996 & 1998 & Mexico & Armed Assault & Private Citizens \& Property \\
\hline Justice for Islamic Movement (JFIM) & Nationalist & 2013 & - & Philippines & Bombing/Explosion & Military, Police \\
\hline $\begin{array}{l}\text { Kabardino-Balkarian Islamic Fighting } \\
\text { Jamaat }\end{array}$ & Nationalist & 2002 & - & Russia & $\begin{array}{l}\text { Armed Assault, Assassination, } \\
\text { Bombing/Explosion }\end{array}$ & Businesses, Police, Utilities \\
\hline Kamal Boulander Group & Religious & 1997 & 1997 & Algeria & Assassination & Private Citizens \& Property \\
\hline $\begin{array}{l}\text { Kamatpur Liberation Organization } \\
\text { (KLO) }\end{array}$ & Nationalist & 1995 & - & India & $\begin{array}{l}\text { Armed Assault, Bombing/Explosion, } \\
\text { Hostage Taking }\end{array}$ & $\begin{array}{l}\text { Businesses, Educational Institutes, } \\
\text { Government (General), Police, Private } \\
\text { Citizens \& Property, Transportation, } \\
\text { Violent Political Party }\end{array}$ \\
\hline Kameradschaft Aachener Land & $\begin{array}{l}\text { Right- } \\
\text { Wing }\end{array}$ & 2011 & - & Germany & Unknown & Private Citizens \& Property \\
\hline
\end{tabular}




\begin{tabular}{|c|c|c|c|c|c|c|}
\hline $\begin{array}{l}\text { VEO Name } \\
\end{array}$ & Type & Emerged & Ended & Countries & $\begin{array}{c}\text { Type of Attack } \\
\end{array}$ & $\begin{array}{c}\text { Target Type } \\
\end{array}$ \\
\hline Kanglei Yawol Kanna Lup (KYKL) & Nationalist & 1994 & - & India & $\begin{array}{l}\text { Armed Assault, Bombing/Explosion, } \\
\text { Hijacking, Hostage Taking }\end{array}$ & $\begin{array}{l}\text { Educational Institutes, Military, Police, } \\
\text { Private Citizens \& Property, Religious } \\
\text { Figures/Institutions, Transportation }\end{array}$ \\
\hline Karbala Brigades & Nationalist & 2004 & 2004 & Iraq & Bombing/Explosion & Businesses \\
\hline $\begin{array}{l}\text { Karbi Longri National Liberation Front } \\
\text { (KLNLF) }\end{array}$ & Nationalist & 2007 & - & India & $\begin{array}{l}\text { Armed Assault, Bombing/Explosion, } \\
\text { Hostage Taking }\end{array}$ & $\begin{array}{l}\text { Businesses, Government (General), } \\
\text { Military, Police, Private Citizens \& } \\
\text { Property, Transportation }\end{array}$ \\
\hline $\begin{array}{l}\text { Karbi Longri North Cachar Hills } \\
\text { Resistance Force }\end{array}$ & Nationalist & 2004 & - & India & $\begin{array}{l}\text { Armed Assault, Bombing/Explosion, } \\
\text { Hostage Taking }\end{array}$ & $\begin{array}{l}\text { Government (General), Military, Police, } \\
\text { Private Citizens \& Property, } \\
\text { Transportation }\end{array}$ \\
\hline Karbi People's Liberation Tigers (KPLT) & Nationalist & 2011 & - & India & $\begin{array}{l}\text { Armed Assault, Bombing/Explosion, } \\
\text { Hostage Taking }\end{array}$ & $\begin{array}{l}\text { Businesses, Government (General), } \\
\text { Police, Private Citizens \& Property, } \\
\text { Terrorists/Non-State Militia, } \\
\text { Transportation }\end{array}$ \\
\hline Karbi Tribe & Nationalist & 1999 & 2005 & India & Bombing/Explosion & Private Citizens \& Property \\
\hline Karwan-e-Niamatullah & Religious & 2007 & - & Pakistan & $\begin{array}{l}\text { Armed Assault, Bombing/Explosion, } \\
\text { Hostage Taking }\end{array}$ & $\begin{array}{l}\text { Educational Institutes, Government } \\
\text { (General), Military }\end{array}$ \\
\hline Kashmir Freedom Force & Nationalist & 2003 & 2003 & India, Pakistan & Bombing/Explosion & Telecommunication \\
\hline Kata'ib al-Ghadab & Nationalist & 2014 & - & Iraq & Armed Assault & Terrorists/Non-State Militia \\
\hline Kata'ib al-Khoul & Nationalist & 2006 & - & Russia & $\begin{array}{l}\text { Armed Assault, Assassination, } \\
\text { Bombing/Explosion }\end{array}$ & $\begin{array}{l}\text { Businesses, Government (General), } \\
\text { Police }\end{array}$ \\
\hline Kata'ib Hizballah & Religious & 2001 & - & Iraq, Syria & $\begin{array}{l}\text { Armed Assault, Bombing/Explosion, } \\
\text { Hostage Taking }\end{array}$ & $\begin{array}{l}\text { Government (Diplomatic), Government } \\
\text { (General), Military, Police, } \\
\text { Terrorists/Non-State Militia }\end{array}$ \\
\hline Kata'ib Sayyid al-Shuhada (KSS) & Religious & 2013 & - & Iraq, Syria & Armed Assault & Terrorists/Non-State Militia \\
\hline Katiba Ibaad al-Rahmaan & Religious & 2016 & - & Syria & $\begin{array}{l}\text { Armed Assault, Assassination, } \\
\text { Bombing/Explosion }\end{array}$ & $\begin{array}{l}\text { Government (General), Military, Private } \\
\text { Citizens \& Property, Religious } \\
\text { Figures/Institutions }\end{array}$ \\
\hline $\begin{array}{l}\text { Katibat al-Aqsa (KAA) - Islamic State } \\
\text { Chechen Unit (ISISC) }\end{array}$ & Religious & 2014 & - & Iraq, Russia, Syria & Unknown & Unknown \\
\hline Katibat al-Mulathamin & Religious & 2004 & - & Algeria, Mali & Unknown & Government (General), Military \\
\hline Katibat al-Tawhid wal Jihad & Religious & 2013 & - & Afghanistan, Pakistan, Syria, Uzbekistan & Armed Assault, Bombing/Explosion & $\begin{array}{l}\text { Government (General), Terrorists/Non- } \\
\text { State Militia }\end{array}$ \\
\hline Katibat Moussaab bin Omeir & Nationalist & 2013 & - & Syria & Unknown & Military \\
\hline Kawal ng Pilipinas & Nationalist & 2006 & 2006 & Philippines & Bombing/Explosion & Government (General) \\
\hline Kenkoku Giyugun Chosen Seibatsutai & $\begin{array}{l}\text { Right- } \\
\text { Wing }\end{array}$ & 2002 & 2004 & Japan & Armed Assault, Bombing/Explosion & Government (General) \\
\hline
\end{tabular}




\begin{tabular}{|c|c|c|c|c|c|c|}
\hline VEO Name & Type & Emerged & Ended & Countries & Type of Attack & Target Type \\
\hline Kenya National Patriotic Front & Nationalist & 1995 & - & Kenya & Unknown & Government (General) \\
\hline Khalifa Islamic Mindanao (KIM) & Nationalist & 2013 & - & Indonesia, Philippines & Armed Assault, Bombing/Explosion & Military, Private Citizens \& Property \\
\hline Khaliq Haqqani Group & Religious & 2007 & - & Pakistan & Armed Assault & Government (General), Military \\
\hline Khilafatul Muslimin & Religious & 1997 & - & Indonesia & Unknown & Government (General) \\
\hline Khorasan Chapter of the Islamic State & Religious & 2014 & - & Afghanistan, Pakistan & $\begin{array}{l}\text { Armed Assault, Assassination, } \\
\text { Bombing/Explosion, } \\
\text { Facility/Infrastructure Attack, Hostage } \\
\text { Taking }\end{array}$ & $\begin{array}{l}\text { Businesses, Educational Institutes, } \\
\text { Government (Diplomatic), Government } \\
\text { (General), Journalists \& Media, Military, } \\
\text { NGO, Police, Private Citizens \& } \\
\text { Property, Religious Figures/Institutions, } \\
\text { Terrorists/Non-State Militia, } \\
\text { Transportation }\end{array}$ \\
\hline $\begin{array}{l}\text { Kizilyurt Cell Caucasus Emirate/Abu } \\
\text { Dujana Brigade }\end{array}$ & Religious & 1998 & - & Russia & Bombing/Explosion & $\begin{array}{l}\text { Military, Private Citizens \& Property, } \\
\text { Religious Figures/Institutions }\end{array}$ \\
\hline Knights of the Tempest & Nationalist & 2005 & - & Palestinian State & Hostage Taking & Government (General) \\
\hline $\begin{array}{l}\text { Koch-Rajbongshi Liberation } \\
\text { Organisation (KRLO) }\end{array}$ & Nationalist & 1995 & 1996 & Bhutan, India & Unknown & Unknown \\
\hline $\begin{array}{l}\text { Komite Persiapan Penegakan Syariat } \\
\text { Islam (KPPSI) }\end{array}$ & Religious & 2000 & - & Indonesia & Bombing/Explosion & Businesses, Military, Tourists \\
\hline Kosovo Liberation Army (KLA) & Nationalist & 1992 & 1999 & Kosovo, Macedonia, Yugoslavia & $\begin{array}{l}\text { Armed Assault, Assassination, } \\
\text { Bombing/Explosion, Hostage Taking }\end{array}$ & $\begin{array}{l}\text { Businesses, Educational Institutes, } \\
\text { Government (Diplomatic), Government } \\
\text { (General), Journalists \& Media, Military, } \\
\text { Police, Private Citizens \& Property, } \\
\text { Transportation }\end{array}$ \\
\hline Kuddam ul-Islam (KuL) & Religious & 2000 & - & India, Pakistan & Armed Assault & Military \\
\hline Kuki Defence Force & Nationalist & 1994 & 1994 & India & Hostage Taking & Government (General) \\
\hline Kuki Liberation Army (KLA) & Nationalist & 1998 & 2005 & India & Bombing/Explosion, Hostage Taking & NGO, Police \\
\hline Kuki Revolutionary Army (KRA) & Nationalist & 1999 & 2007 & India & Armed Assault, Hostage Taking & $\begin{array}{l}\text { Government (General), Military, Private } \\
\text { Citizens \& Property, Violent Political } \\
\text { Party }\end{array}$ \\
\hline Kumpulan Mujahidin Malaysia & Religious & 1995 & 2014 & Indonesia, Malaysia, Nepal, Philippines & $\begin{array}{l}\text { Assassination, Bombing/Explosion, } \\
\text { Hostage Taking }\end{array}$ & $\begin{array}{l}\text { Government (General), Religious } \\
\text { Figures/Institutions }\end{array}$ \\
\hline $\begin{array}{l}\text { Kunama Democratic Liberation } \\
\text { Movement (EKDLM) }\end{array}$ & Nationalist & 1998 & - & Eritrea, Ethiopia & Unknown & Government (General) \\
\hline Kurdish Communities Union (KCK) & Nationalist & 2005 & - & Iraq, Turkey & Armed Assault, Bombing/Explosion & Government (General) \\
\hline Kurdish Islamic Group (KIG) & Religious & 2001 & - & Iraq & Armed Assault, Assassination & $\begin{array}{l}\text { Government (General), Private Citizens } \\
\text { \& Property }\end{array}$ \\
\hline Kurdish Islamic Unity Party & Nationalist & 1995 & 1995 & Turkey & Bombing/Explosion & Tourists \\
\hline
\end{tabular}




\begin{tabular}{|c|c|c|c|c|c|c|}
\hline VEO Name & Type & Emerged & Ended & Countries & Type of Attack & Target Type \\
\hline Kurdish Patriotic Union & Nationalist & 1994 & 1994 & Turkey & Unknown & Unknown \\
\hline Kurdistan Free Life Party & Left-Wing & 2004 & - & Iran & Armed Assault, Assassination & Government (General), Military, Police \\
\hline Kurdistan Freedom Hawks (TAK) & Nationalist & 2004 & - & Iran, Turkey & Bombing/Explosion & $\begin{array}{l}\text { Airports \& Aircraft, Businesses, } \\
\text { Government (General), Military, Police, } \\
\text { Private Citizens \& Property, Religious } \\
\text { Figures/Institutions, Tourists, Utilities }\end{array}$ \\
\hline Kurdistan Islamic Union (KIU) & Nationalist & 1994 & - & Iran, Iraq, Syria, Turkey & Unknown & Government (General) \\
\hline Lahij Province of the Islamic State & Religious & 2015 & - & Yemen & Assassination, Hostage Taking & Government (General), Military \\
\hline Lashkar-e-Balochistan & Nationalist & 2009 & - & Pakistan & $\begin{array}{l}\text { Armed Assault, Bombing/Explosion, } \\
\text { Facility/Infrastructure Attack }\end{array}$ & $\begin{array}{l}\text { Businesses, Government (Diplomatic), } \\
\text { Government (General), Military, Police, } \\
\text { Private Citizens \& Property, Religious } \\
\text { Figures/Institutions, Terrorists/Non- } \\
\text { State Militia, Transportation }\end{array}$ \\
\hline Lashkar-e-Fidayan-e-Islam & Religious & 2003 & - & Pakistan & Bombing/Explosion & Military \\
\hline Lashkar-e-Isar & Nationalist & 1992 & 1997 & Afghanistan & Bombing/Explosion & $\begin{array}{l}\text { Private Citizens \& Property, } \\
\text { Terrorists/Non-State Militia }\end{array}$ \\
\hline Lashkar-e-Islam & Religious & 2004 & - & India, Pakistan & $\begin{array}{l}\text { Armed Assault, Bombing/Explosion, } \\
\text { Facility/Infrastructure Attack, } \\
\text { Hijacking, Hostage Taking }\end{array}$ & $\begin{array}{l}\text { Businesses, Educational Institutes, } \\
\text { Government (General), Military, Police, } \\
\text { Private Citizens \& Property, Religious } \\
\text { Figures/Institutions, } \\
\text { Telecommunication, Terrorists/Non- } \\
\text { State Militia }\end{array}$ \\
\hline Lashkar-e-Jabbar & Religious & 2001 & - & India & Armed Assault, Unarmed Assault & $\begin{array}{l}\text { Private Citizens \& Property, Religious } \\
\text { Figures/Institutions }\end{array}$ \\
\hline Lashkar-e-Jhangvi & Religious & 1996 & - & Afghanistan, India, Pakistan & $\begin{array}{l}\text { Armed Assault, Assassination, } \\
\text { Bombing/Explosion, Hostage Taking, } \\
\text { Unarmed Assault }\end{array}$ & $\begin{array}{l}\text { Businesses, Educational Institutes, } \\
\text { Government (General), Journalists \& } \\
\text { Media, Military, Police, Private Citizens } \\
\text { \& Property, Religious } \\
\text { Figures/Institutions, Transportation, } \\
\text { Violent Political Party }\end{array}$ \\
\hline Lashkar-e-Kahar & Nationalist & 2006 & - & India & Bombing/Explosion & Government (General) \\
\hline Lashkar-I-Omar & Religious & 2001 & - & Pakistan & Armed Assault, Bombing/Explosion & $\begin{array}{l}\text { Government (Diplomatic), Religious } \\
\text { Figures/Institutions }\end{array}$ \\
\hline Laskar Jihad & Religious & 2000 & - & Indonesia & Armed Assault, Bombing/Explosion & Private Citizens \& Property \\
\hline Laskar Jundullah & Religious & 2000 & 2002 & Indonesia & Bombing/Explosion & Businesses, Private Citizens \& Property \\
\hline Laskar Khalid bin Walid & Religious & 2000 & - & Indonesia & Armed Assault & $\begin{array}{l}\text { Private Citizens \& Property, Religious } \\
\text { Figures/Institutions }\end{array}$ \\
\hline Last Generation & Left-Wing & 2000 & 2000 & Greece & Bombing/Explosion & Private Citizens \& Property \\
\hline Latin American Patriotic Army & Left-Wing & 2001 & 2001 & Colombia, Venezuela & Hostage Taking & Businesses \\
\hline
\end{tabular}




\begin{tabular}{|c|c|c|c|c|c|c|}
\hline VEO Name & Type & Emerged & Ended & Countries & Type of Attack & Target Type \\
\hline League of Bahraini Resistance (AMB) & Religious & 2014 & - & Bahrain, Iran & Bombing/Explosion & Government (General) \\
\hline Lega Nord/Northern League - Venice & Nationalist & 2014 & - & Italy & Armed Assault, Hostage Taking & Government (General) \\
\hline $\begin{array}{l}\text { Lehava/Organization for Prevention of } \\
\text { Assimilation in the Holy Land }\end{array}$ & $\begin{array}{l}\text { Right- } \\
\text { Wing }\end{array}$ & 2014 & - & Israel & Facility/Infrastructure Attack & Educational Institutes \\
\hline $\begin{array}{l}\text { Liberation and Independence of the } \\
\text { Sangha-Mbaere (FLISM) }\end{array}$ & Religious & 2013 & - & The Central African Republic & Armed Assault & $\begin{array}{l}\text { Government (General), Terrorists/Non- } \\
\text { State Militia }\end{array}$ \\
\hline Liberation and Justice Movement (LJM) & Nationalist & 2010 & - & Sudan & Armed Assault, Hostage Taking & $\begin{array}{l}\text { Government (General), Military, Police, } \\
\text { Private Citizens \& Property }\end{array}$ \\
\hline $\begin{array}{l}\text { Liberation Army for Presevo, Medvedja, } \\
\text { and Bujanovac (UCPMB) }\end{array}$ & Nationalist & 2001 & 2001 & Kosovo & Assassination, Bombing/Explosion & $\begin{array}{l}\text { Government (Diplomatic), Military, } \\
\text { Police, Private Citizens \& Property }\end{array}$ \\
\hline Liberation of Achik Elite Force (LAEF) & Nationalist & 2005 & - & India & Armed Assault, Hostage Taking & $\begin{array}{l}\text { Government (General), Private Citizens } \\
\text { \& Property }\end{array}$ \\
\hline Liberia Peace Council & Nationalist & 1993 & 1996 & Cote D'Ivoire, Liberia & Armed Assault & Private Citizens \& Property \\
\hline $\begin{array}{l}\text { Liberians United for Reconciliation and } \\
\text { Democracy (LURD) }\end{array}$ & Nationalist & 1999 & 2003 & Liberia & $\begin{array}{l}\text { Armed Assault, Assassination, Hostage } \\
\text { Taking }\end{array}$ & $\begin{array}{l}\text { Government (General), NGO, Private } \\
\text { Citizens \& Property, Religious } \\
\text { Figures/Institutions }\end{array}$ \\
\hline Libya Dawn & Religious & 2014 & - & Libya & Armed Assault, Bombing/Explosion & $\begin{array}{l}\text { Government (General), Terrorists/Non- } \\
\text { State Militia, Utilities }\end{array}$ \\
\hline $\begin{array}{l}\text { Libya Revolutionaries Joint Operations } \\
\text { Room (LROR) }\end{array}$ & Nationalist & 2013 & - & Libya & $\begin{array}{l}\text { Armed Assault, Bombing/Explosion, } \\
\text { Hostage Taking }\end{array}$ & $\begin{array}{l}\text { Airports \& Aircraft, Government } \\
\text { (General), Military, Private Citizens \& } \\
\text { Property }\end{array}$ \\
\hline Libyan Islamic Fighting Group (LIFG) & Religious & 1995 & - & Iran, Libya, United Kingdom & $\begin{array}{l}\text { Armed Assault, Assassination, } \\
\text { Bombing/Explosion }\end{array}$ & $\begin{array}{l}\text { Government (General), Military, Private } \\
\text { Citizens \& Property }\end{array}$ \\
\hline Libyan Shield Force (LSF) & Nationalist & 2012 & - & Libya & $\begin{array}{l}\text { Armed Assault, Bombing/Explosion, } \\
\text { Facility/Infrastructure Attack, Hostage } \\
\text { Taking }\end{array}$ & $\begin{array}{l}\text { Government (Diplomatic), Military, } \\
\text { Private Citizens \& Property }\end{array}$ \\
\hline Lions Macedonia & Nationalist & 2001 & & $\begin{array}{l}\text { Bulgaria, Croatia, Macedonia, Russia, } \\
\text { Serbia, Ukraine }\end{array}$ & Armed Assault, Hostage Taking & Government (General), NGO \\
\hline Liwa Ahrar al-Sunna & Religious & 2014 & - & Israel, Lebanon & Armed Assault, Bombing/Explosion & $\begin{array}{l}\text { Businesses, Military, Police, Private } \\
\text { Citizens \& Property, Telecommunication }\end{array}$ \\
\hline Liwa al-Fath & Nationalist & 2012 & - & Syria, Turkey & Armed Assault & Military, Terrorists/Non-State Militia \\
\hline Liwa al-Haqq & Nationalist & 2012 & - & Syria & Armed Assault, Bombing/Explosion & Military \\
\hline Liwa al-Islam & Religious & 2013 & - & Syria & Bombing/Explosion & Military \\
\hline Liwa al-Sham & Religious & 2013 & - & Syria & Bombing/Explosion & Military \\
\hline Liwa al-Tawhid & Religious & 2012 & - & Syria & $\begin{array}{l}\text { Armed Assault, Bombing/Explosion, } \\
\text { Hostage Taking }\end{array}$ & $\begin{array}{l}\text { Government (General), Journalists \& } \\
\text { Media, Military }\end{array}$ \\
\hline
\end{tabular}




\begin{tabular}{|c|c|c|c|c|c|c|}
\hline VEO Name & Type & Emerged & Ended & Countries & Type of Attack & Target Type \\
\hline Liwa Thuwar Raqqa & Nationalist & 2013 & - & Syria & Armed Assault & Terrorists/Non-State Militia \\
\hline Liwa'a Abu Fadl al-Abbas (LAFA) & Religious & 2012 & - & Iran, Iraq, Lebanon, Syria & Bombing/Explosion & Terrorists/Non-State Militia \\
\hline Liwa'a al-Umma & Nationalist & 2011 & - & Libya, Syria & Armed Assault & Military \\
\hline Liwa'a 'Ammar Ibn Yasir (LAIY) & Religious & 2013 & - & Syria & Armed Assault, Bombing/Explosion & Terrorists/Non-State Militia \\
\hline Loyalist Action Force & Religious & 2001 & - & Ireland, United Kingdom & Armed Assault, Bombing/Explosion & $\begin{array}{l}\text { Private Citizens \& Property, Religious } \\
\text { Figures/Institutions }\end{array}$ \\
\hline Loyalist Volunteer Force & Nationalist & 1997 & 2003 & Ireland, United Kingdom & $\begin{array}{l}\text { Armed Assault, Assassination, } \\
\text { Bombing/Explosion, } \\
\text { Facility/Infrastructure Attack, Hostage } \\
\text { Taking }\end{array}$ & $\begin{array}{l}\text { Businesses, Government (General), } \\
\text { Private Citizens \& Property, Religious } \\
\text { Figures/Institutions, Terrorists/Non- } \\
\text { State Militia }\end{array}$ \\
\hline Luhansk People's Republic & Nationalist & 2014 & - & Ukraine & $\begin{array}{l}\text { Armed Assault, Assassination, } \\
\text { Bombing/Explosion, } \\
\text { Facility/Infrastructure Attack, Hostage } \\
\text { Taking }\end{array}$ & $\begin{array}{l}\text { Airports \& Aircraft, Businesses, Food or } \\
\text { Water Supply, Government } \\
\text { (Diplomatic), Government (General), } \\
\text { Journalists \& Media, Military, Police, } \\
\text { Private Citizens \& Property, } \\
\text { Terrorists/Non-State Militia, } \\
\text { Transportation, Utilities }\end{array}$ \\
\hline LW-Toulouse & $\begin{array}{l}\text { Right- } \\
\text { Wing }\end{array}$ & 2008 & - & France & Bombing/Explosion & Religious Figures/Institutions \\
\hline M23 & Nationalist & 2012 & - & Congo, Democratic Republic, Rwanda & Armed Assault, Bombing/Explosion & $\begin{array}{l}\text { Government (Diplomatic), Government } \\
\text { (General), Military, Private Citizens \& } \\
\text { Property }\end{array}$ \\
\hline Macedonia Dawn & Nationalist & 1999 & 1999 & Macedonia & Bombing/Explosion & Military \\
\hline Macedonian Revolutionary Organization & Nationalist & 2001 & 2001 & Greece, Macedonia & Unknown & $\begin{array}{l}\text { Government (General), Private Citizens } \\
\text { \& Property }\end{array}$ \\
\hline Macina Liberation Front (FLM) & Religious & 2015 & - & Mali & $\begin{array}{l}\text { Armed Assault, Bombing/Explosion, } \\
\text { Facility/Infrastructure Attack, } \\
\text { Hijacking, Hostage Taking }\end{array}$ & $\begin{array}{l}\text { Government (General), Military, Police, } \\
\text { Private Citizens \& Property, Religious } \\
\text { Figures/Institutions }\end{array}$ \\
\hline Madhesi Liberation Front & Left-Wing & 2001 & - & Nepal & Bombing/Explosion & $\begin{array}{l}\text { Businesses, Government (General), } \\
\text { Private Citizens \& Property }\end{array}$ \\
\hline Madhesi Mukti Tigers (MMT) & Nationalist & 2008 & - & Nepal & $\begin{array}{l}\text { Armed Assault, Assassination, } \\
\text { Bombing/Explosion }\end{array}$ & $\begin{array}{l}\text { Businesses, Government (General), } \\
\text { Private Citizens \& Property, Violent } \\
\text { Political Party }\end{array}$ \\
\hline Madhesi People's Rights Forum (MPRF) & Left-Wing & 2007 & - & Nepal & $\begin{array}{l}\text { Armed Assault, Bombing/Explosion, } \\
\text { Unarmed Assault }\end{array}$ & $\begin{array}{l}\text { Businesses, Government (General), } \\
\text { Private Citizens \& Property }\end{array}$ \\
\hline Madhesi Virus Killers & Nationalist & 2008 & - & Nepal & Bombing/Explosion & Transportation \\
\hline
\end{tabular}




\begin{tabular}{|c|c|c|c|c|c|c|}
\hline VEO Name & Type & Emerged & Ended & Countries & Type of Attack & Target Type \\
\hline $\begin{array}{l}\text { Mahan Madhesh Janakantri Party } \\
\text { (MMJP) }\end{array}$ & Nationalist & 2009 & - & Nepal & Bombing/Explosion & $\begin{array}{l}\text { Businesses, Government (General), } \\
\text { Journalists \& Media, Private Citizens \& } \\
\text { Property }\end{array}$ \\
\hline Mahaz-e-Inquilab & Religious & 2005 & 2005 & India & Bombing/Explosion & $\begin{array}{l}\text { Private Citizens \& Property, } \\
\text { Transportation }\end{array}$ \\
\hline Mahdaviyat & Religious & 1999 & 1999 & Iran & Assassination & Government (General) \\
\hline Mahdi Army & Nationalist & 2003 & - & Iran, Iraq & $\begin{array}{l}\text { Armed Assault, Assassination, } \\
\text { Bombing/Explosion, Hostage Taking }\end{array}$ & $\begin{array}{l}\text { Businesses, Government (Diplomatic), } \\
\text { Government (General), NGO, Police, } \\
\text { Private Citizens \& Property, } \\
\text { Terrorists/Non-State Militia }\end{array}$ \\
\hline Mai Mai Bakata Katanga Militia & Nationalist & 2014 & - & Congo, Democratic Republic & $\begin{array}{l}\text { Facility/Infrastructure Attack, Hostage } \\
\text { Taking }\end{array}$ & $\begin{array}{l}\text { Government (General), Military, Police, } \\
\text { Private Citizens \& Property }\end{array}$ \\
\hline Mai Mai Mazembe Militia & Nationalist & 2016 & - & Congo, Democratic Republic & $\begin{array}{l}\text { Armed Assault, Facility/Infrastructure } \\
\text { Attack }\end{array}$ & Military, Private Citizens \& Property \\
\hline Mai Mai Simba Militia & $\begin{array}{l}\text { Right- } \\
\text { Wing }\end{array}$ & 2012 & - & Congo, Democratic Republic & Armed Assault, Hostage Taking & $\begin{array}{l}\text { Businesses, Military, Private Citizens \& } \\
\text { Property }\end{array}$ \\
\hline Majelis Mujahidin Indonesia (MMI) & Religious & 2000 & - & Indonesia & Bombing/Explosion & $\begin{array}{l}\text { Businesses, Private Citizens \& Property, } \\
\text { Religious Figures/Institutions, Tourists }\end{array}$ \\
\hline Majelis Zikir Nurhaerat Poso & Religious & 2001 & - & Indonesia & Armed Assault & Private Citizens \& Property \\
\hline Majlis Shura al-Mujahedeen (MSM) & Religious & 2012 & - & Israel, Palestinian State & Bombing/Explosion & Government (General) \\
\hline Makhachkala Terrorists & Religious & 2005 & - & Russia & Bombing/Explosion & Government (General), Police \\
\hline Malaita Eagles Force & Nationalist & 1999 & - & Solomon Islands & Armed Assault, Hostage Taking & $\begin{array}{l}\text { Government (General), Violent Political } \\
\text { Party }\end{array}$ \\
\hline $\begin{array}{l}\text { Malhama Tactical (JFS/Conquest of al- } \\
\text { Sham Front) }\end{array}$ & Religious & 2015 & - & Syria, Uzbekistan & Armed Assault & Government (General) \\
\hline Maluku Sovereignty Front & Nationalist & 2000 & - & Indonesia, Netherlands & Armed Assault, Bombing/Explosion & Military, Private Citizens \& Property \\
\hline Manba al-Jihad (MAJ) & Religious & 2008 & - & Afghanistan & Unknown & Journalists \& Media \\
\hline Manuel Ascencio Padilla Group & Left-Wing & 1992 & 1992 & Bolivia & Bombing/Explosion & Private Citizens \& Property \\
\hline Maoist Communist Party (MKP) & Left-Wing & 2004 & - & Turkey & Armed Assault, Assassination & $\begin{array}{l}\text { Government (General), Private Citizens } \\
\text { \& Property }\end{array}$ \\
\hline Maoist Communist Party of Manipur & Left-Wing & 2013 & - & India & Assassination, Bombing/Explosion & Businesses, Police \\
\hline $\begin{array}{l}\text { Maoist Farm Laborers Struggle } \\
\text { Committee (MXSS) }\end{array}$ & Left-Wing & 1994 & 1994 & India & Armed Assault & Private Citizens \& Property \\
\hline $\begin{array}{l}\text { Mariano Moreno National Liberation } \\
\text { Comando }\end{array}$ & Left-Wing & 2005 & 2005 & Argentina & Bombing/Explosion & Businesses \\
\hline
\end{tabular}




\begin{tabular}{|c|c|c|c|c|c|c|}
\hline VEO Name & Type & Emerged & Ended & Countries & Type of Attack & Target Type \\
\hline Martyr Abu-Ali Mustafa Brigades & Nationalist & 2001 & - & Palestinian State & Unknown & $\begin{array}{l}\text { Businesses, Government (General), } \\
\text { Private Citizens \& Property, } \\
\text { Transportation }\end{array}$ \\
\hline Martyrs Brigade - Nigeria & Nationalist & 2005 & - & Nigeria & Bombing/Explosion & Businesses \\
\hline Martyrs of 17 February Brigade & Religious & 2012 & - & Libya & Armed Assault & Private Citizens \& Property \\
\hline Martyrs of Syria Brigade & Nationalist & 2011 & - & Syria & Armed Assault, Bombing/Explosion & Military \\
\hline Marwan Hadid Brigades & Religious & 2013 & - & Lebanon, Syria & Bombing/Explosion & Violent Political Party \\
\hline $\begin{array}{l}\text { Marxist-Leninist Communist Party of } \\
\text { Venezuela (PCMLV) }\end{array}$ & Left-Wing & 2009 & - & Venezuela & Unknown & Unknown \\
\hline $\begin{array}{l}\text { Marxist-Leninist Party of the Philippines } \\
\text { (PMLP) }\end{array}$ & Left-Wing & 2004 & - & Philippines & Armed Assault, Bombing/Explosion & Government (General) \\
\hline Ma'sadat al-Mujahideen & Nationalist & 2008 & - & Israel, Palestinian State, United States & $\begin{array}{l}\text { Bombing/Explosion, } \\
\text { Facility/Infrastructure Attack }\end{array}$ & Businesses, Private Citizens \& Property \\
\hline Masked Battalion & Religious & 2007 & - & Mali & Hostage Taking & Military, Private Citizens \& Property \\
\hline Mateo Morral Commando & Left-Wing & 2013 & - & Chile, Spain & Bombing/Explosion & Religious Figures/Institutions \\
\hline Maute Group/Islamic State of Lanao & Religious & 2015 & - & Indonesia, Philippines & $\begin{array}{l}\text { Armed Assault, Bombing/Explosion, } \\
\text { Hostage Taking }\end{array}$ & $\begin{array}{l}\text { Businesses, Government (Diplomatic), } \\
\text { Military, Police, Private Citizens \& } \\
\text { Property, Utilities }\end{array}$ \\
\hline May 15 & Left-Wing & 1998 & 1998 & Greece & Bombing/Explosion & Airports \& Aircraft, Businesses \\
\hline May 98 & Left-Wing & 1998 & 1998 & Greece & Facility/Infrastructure Attack & Businesses \\
\hline Mayi Mayi & Nationalist & 2001 & - & Congo, Democratic Republic & Armed Assault, Hostage Taking & $\begin{array}{l}\text { Airports \& Aircraft, Businesses, } \\
\text { Educational Institutes, Government } \\
\text { (Diplomatic), Journalists \& Media, } \\
\text { Military, NGO, Police, Private Citizens \& } \\
\text { Property }\end{array}$ \\
\hline Mazdoor Kisan Sangram Samiti (MKSS) & Left-Wing & 1999 & 1999 & India & Armed Assault & Private Citizens \& Property \\
\hline Megafonen & Nationalist & 2008 & - & Sweden & Bombing/Explosion & Government (General), Police \\
\hline Mehsud Group & Nationalist & 2001 & - & Afghanistan, Pakistan & Assassination, Hostage Taking & Military, Terrorists/Non-State Militia \\
\hline Meiliani Group & Religious & 2001 & - & Germany, Italy, Spain, United Kingdom & Bombing/Explosion & $\begin{array}{l}\text { Government (General), Religious } \\
\text { Figures/Institutions }\end{array}$ \\
\hline Melting Nuclei & Left-Wing & 1998 & 1998 & Greece & Bombing/Explosion & Businesses \\
\hline Midnight Saboteurs & Left-Wing & 2004 & 2004 & Greece & Bombing/Explosion & Businesses \\
\hline Militant Minority & Left-Wing & 2012 & - & Greece & $\begin{array}{l}\text { Bombing/Explosion, } \\
\text { Facility/Infrastructure Attack }\end{array}$ & $\begin{array}{l}\text { Government (General), Journalists \& } \\
\text { Media }\end{array}$ \\
\hline $\begin{array}{l}\text { Militant Movement for Madagascan } \\
\text { Socialism (MMSM) }\end{array}$ & Left-Wing & 1992 & 1992 & Madagascar & Armed Assault & Government (General) \\
\hline
\end{tabular}




\begin{tabular}{|c|c|c|c|c|c|c|}
\hline VEO Name & Type & Emerged & Ended & Countries & Type of Attack & Target Type \\
\hline $\begin{array}{l}\text { Militant Organization of Russian } \\
\text { Nationalists }\end{array}$ & Nationalist & 2008 & - & Russia & Armed Assault & Private Citizens \& Property \\
\hline Militante Gruppe & Left-Wing & 2001 & 2007 & Germany & Bombing/Explosion & Government (General), Police \\
\hline Militants of the National Action Party & Religious & 1992 & 1992 & Mexico & Facility/Infrastructure Attack & Government (General) \\
\hline $\begin{array}{l}\text { Military Command Council for the } \\
\text { Wellbeing of the Republic (CCMSR) - } \\
\text { Chad }\end{array}$ & Nationalist & 2016 & - & Chad & Armed Assault & Military \\
\hline $\begin{array}{l}\text { Military Council of the Tribal } \\
\text { Revolutionaries (MCTR) }\end{array}$ & Nationalist & 2014 & - & Iraq & Armed Assault, Bombing/Explosion & $\begin{array}{l}\text { Airports \& Aircraft, Educational } \\
\text { Institutes, Military, Police, Private } \\
\text { Citizens \& Property }\end{array}$ \\
\hline Misrata al-Nusra Brigade & Nationalist & 2013 & - & Libya & Armed Assault & Private Citizens \& Property \\
\hline Misrata Brigades (MUR) & Nationalist & 2011 & - & Libya & $\begin{array}{l}\text { Armed Assault, Bombing/Explosion, } \\
\text { Hijacking, Hostage Taking }\end{array}$ & $\begin{array}{l}\text { Businesses, Government (Diplomatic), } \\
\text { Government (General), Military, Police, } \\
\text { Private Citizens \& Property }\end{array}$ \\
\hline Misuari Renegade Group (MRG) & Nationalist & 2001 & - & Philippines & $\begin{array}{l}\text { Armed Assault, Bombing/Explosion, } \\
\text { Hostage Taking }\end{array}$ & Businesses, Military, Transportation \\
\hline Mlada Bosna & Nationalist & 2008 & - & Montenegro, Serbia & Bombing/Explosion & Government (Diplomatic), NGO \\
\hline Mohammed's Army & Religious & 2000 & 2000 & Yemen & Bombing/Explosion & Maritime, Military \\
\hline Mohiuddin al-Nasser Martyrs Brigade & Nationalist & 2013 & - & Iran & Bombing/Explosion & Utilities \\
\hline Mombasa Republican Council (MRC) & Nationalist & 1999 & - & Kenya & Armed Assault & Government (General), Police \\
\hline Moroccan Network & Religious & 2005 & - & $\begin{array}{l}\text { Belgium, Italy, Mauritania, Morocco, } \\
\text { Spain, Tunisia }\end{array}$ & Armed Assault, Bombing/Explosion & $\begin{array}{l}\text { Businesses, Government (General), } \\
\text { Maritime, Religious } \\
\text { Figures/Institutions, Tourists }\end{array}$ \\
\hline Mourabitounes/al-Qaeda in West Africa & Religious & 2013 & - & Algeria, Egypt, Libya, Mali, Niger & Armed Assault, Bombing/Explosion & Businesses, Military, Tourists \\
\hline $\begin{array}{l}\text { Movement for Actualization of the } \\
\text { Sovereign State of Biafra (MASSOB) }\end{array}$ & Nationalist & 2001 & 2007 & Nigeria & Armed Assault, Unarmed Assault & $\begin{array}{l}\text { Journalists \& Media, Police, Religious } \\
\text { Figures/Institutions, } \\
\text { Telecommunication }\end{array}$ \\
\hline $\begin{array}{l}\text { Movement for Democracy and Justice in } \\
\text { Chad (MDJT) }\end{array}$ & Left-Wing & 1998 & 2003 & Chad & Unknown & Military \\
\hline $\begin{array}{l}\text { Movement for Democratic Change } \\
\text { (MDC) }\end{array}$ & Left-Wing & 2002 & - & Zimbabwe & Armed Assault, Bombing/Explosion & $\begin{array}{l}\text { Businesses, Government (General), } \\
\text { Violent Political Party }\end{array}$ \\
\hline Movement for Dignity and Sovereignty & Nationalist & 1994 & 1994 & Nicaragua & Hostage Taking & Government (Diplomatic) \\
\hline $\begin{array}{l}\text { Movement for Self-Determination } \\
\text { (MPA) }\end{array}$ & Nationalist & 1995 & 1995 & France & Assassination & Violent Political Party \\
\hline $\begin{array}{l}\text { Movement for the Emancipation of the } \\
\text { Niger Delta (MEND) }\end{array}$ & Nationalist & 2005 & - & Nigeria & $\begin{array}{l}\text { Armed Assault, Bombing/Explosion, } \\
\text { Facility/Infrastructure Attack, Hostage } \\
\text { Taking }\end{array}$ & $\begin{array}{l}\text { Airports \& Aircraft, Businesses, } \\
\text { Government (General), Maritime, } \\
\text { Military, Police, Private Citizens \& } \\
\text { Property, Utilities, Violent Political } \\
\text { Party }\end{array}$ \\
\hline
\end{tabular}




\begin{tabular}{|c|c|c|c|c|c|c|}
\hline VEO Name & Type & Emerged & Ended & Countries & Type of Attack & Target Type \\
\hline Movement for the Liberation of Congo & Left-Wing & 1996 & 2003 & Congo, Democratic Republic, Uganda & Armed Assault & Maritime, Military \\
\hline $\begin{array}{l}\text { Movement for the Unity and Jihad in } \\
\text { West Africa (MUJAO)/Movement for } \\
\text { Oneness and Jihad in West Africa } \\
\text { (MUJAWA) }\end{array}$ & Religious & 2011 & - & Algeria, Mali, Mauritania, Niger & $\begin{array}{l}\text { Armed Assault, Assassination, } \\
\text { Bombing/Explosion, Hostage Taking }\end{array}$ & $\begin{array}{l}\text { Businesses, Government (Diplomatic), } \\
\text { Government (General), Military, NGO, } \\
\text { Police, Private Citizens \& Property, } \\
\text { Religious Figures/Institutions, } \\
\text { Transportation }\end{array}$ \\
\hline $\begin{array}{l}\text { Movement of Sons of the Sahara for } \\
\text { Islamic Justice (MSIJ) }\end{array}$ & Nationalist & 2007 & - & Algeria, Libya, Niger & Bombing/Explosion, Hostage Taking & $\begin{array}{l}\text { Airports \& Aircraft, Military, NGO, } \\
\text { Utilities }\end{array}$ \\
\hline $\begin{array}{l}\text { Movement of the Democratic Forces of } \\
\text { Guinea (RFDG) }\end{array}$ & Nationalist & 2000 & - & Guinea & Armed Assault, Bombing/Explosion & $\begin{array}{l}\text { Government (General), Private Citizens } \\
\text { \& Property }\end{array}$ \\
\hline Movement of the Islamic State (MEI) & Religious & 1994 & 1994 & Algeria & Assassination & Government (General) \\
\hline Movsar Baryayev Gang & Nationalist & 1998 & 2002 & Russia & Assassination, Hostage Taking & $\begin{array}{l}\text { Government (General), NGO, Private } \\
\text { Citizens \& Property }\end{array}$ \\
\hline Muhajideen Army of Aleppo & Religious & 2014 & - & Syria & Armed Assault, Bombing/Explosion & Military, Terrorists/Non-State Militia \\
\hline $\begin{array}{l}\text { Muhajireen Brigade/Jaish al- } \\
\text { Muhajireen wal-Ansar }\end{array}$ & Religious & 2012 & - & Russia, Syria & Armed Assault, Bombing/Explosion & Government (General), Military \\
\hline $\begin{array}{l}\text { Mujahadi Bayt al-Maqdis Brigades } \\
\text { (JAMB) }\end{array}$ & Religious & 2005 & - & Egypt, Israel, Palestinian State & $\begin{array}{l}\text { Armed Assault, Assassination, } \\
\text { Bombing/Explosion, Hostage Taking }\end{array}$ & $\begin{array}{l}\text { Government (General), Military, Private } \\
\text { Citizens \& Property }\end{array}$ \\
\hline Mujahedeen Brigades & Religious & 2004 & 2004 & Iraq & Hostage Taking & $\begin{array}{l}\text { Government (General), Private Citizens } \\
\text { \& Property }\end{array}$ \\
\hline Mujahedeen Corps in Iraq & Religious & 2004 & - & Iraq & Bombing/Explosion, Hostage Taking & Businesses, Government (Diplomatic) \\
\hline Mujahideen al-Mansooran & Nationalist & 2002 & 2002 & India & Armed Assault & $\begin{array}{l}\text { Government (General), Private Citizens } \\
\text { \& Property }\end{array}$ \\
\hline Mujahideen Army & Religious & 2004 & - & Iraq & Assassination, Bombing/Explosion & $\begin{array}{l}\text { Military, Private Citizens \& Property, } \\
\text { Religious Figures/Institutions }\end{array}$ \\
\hline Mujahideen Brigades in Eritrea & Religious & 2005 & - & Eritrea & Armed Assault & Businesses, Government (General) \\
\hline Mujahideen Division Khandaq & Religious & 2000 & 2000 & Indonesia, Malaysia & Bombing/Explosion & Government (Diplomatic) \\
\hline Mujahideen in Lebanon & Religious & 2006 & - & Lebanon & Armed Assault & Private Citizens \& Property \\
\hline Mujahideen Islam Pattani & Religious & 2002 & 2004 & Tanzania & Armed Assault, Bombing/Explosion & $\begin{array}{l}\text { Educational Institutes, Military, Police, } \\
\text { Transportation }\end{array}$ \\
\hline Mujahideen KOMPAK & Religious & 2000 & - & Indonesia & Armed Assault, Bombing/Explosion & $\begin{array}{l}\text { Educational Institutes, Private Citizens } \\
\text { \& Property, Religious } \\
\text { Figures/Institutions }\end{array}$ \\
\hline Mujahideen Message & Nationalist & 2003 & - & Afghanistan & Bombing/Explosion & Educational Institutes \\
\hline Mujahideen Movement in Morocco & Nationalist & 2003 & - & Morocco & Armed Assault, Assassination & Government (General) \\
\hline
\end{tabular}




\begin{tabular}{|c|c|c|c|c|c|c|}
\hline VEO Name & Type & Emerged & Ended & Countries & Type of Attack & Target Type \\
\hline $\begin{array}{l}\text { Mujahideen of Tatarstan/Mujahideens } \\
\text { of Tatarstan }\end{array}$ & Religious & 2007 & - & Russia & Assassination, Bombing/Explosion & $\begin{array}{l}\text { Government (Diplomatic), Government } \\
\text { (General), Journalists \& Media, Military, } \\
\text { Private Citizens \& Property, Religious } \\
\text { Figures/Institutions }\end{array}$ \\
\hline $\begin{array}{l}\text { Mujahideen of the Caucasus and the } \\
\text { Levant (MCL) }\end{array}$ & Religious & 2013 & - & Syria & Armed Assault & Military \\
\hline Mujahideen Shura Council & Religious & 2005 & - & Iraq, Syria & $\begin{array}{l}\text { Armed Assault, Bombing/Explosion, } \\
\text { Hostage Taking }\end{array}$ & $\begin{array}{l}\text { Government (General), Police, Private } \\
\text { Citizens \& Property }\end{array}$ \\
\hline Mujahideen Youth Movement (MYM) & Religious & 2007 & - & Somalia & $\begin{array}{l}\text { Armed Assault, Assassination, } \\
\text { Bombing/Explosion, } \\
\text { Facility/Infrastructure Attack }\end{array}$ & $\begin{array}{l}\text { Government (Diplomatic), Government } \\
\text { (General), Military, Police, Private } \\
\text { Citizens \& Property }\end{array}$ \\
\hline Mujahideen Indonesia Timur (MIT) & Nationalist & 2010 & - & Indonesia, Philippines & $\begin{array}{l}\text { Armed Assault, Assassination, } \\
\text { Bombing/Explosion, Hostage Taking }\end{array}$ & $\begin{array}{l}\text { Military, Police, Private Citizens \& } \\
\text { Property }\end{array}$ \\
\hline Mujahidin Ambon & Religious & 2005 & 2005 & Indonesia & Armed Assault, Bombing/Explosion & $\begin{array}{l}\text { Businesses, Police, Private Citizens \& } \\
\text { Property }\end{array}$ \\
\hline $\begin{array}{l}\text { Mujahidin Shura Council in the } \\
\text { Environs of Jerusalem (MSC) }\end{array}$ & Nationalist & 2012 & - & Egypt, Israel, Palestinian State & Armed Assault, Bombing/Explosion & $\begin{array}{l}\text { Businesses, Military, Private Citizens \& } \\
\text { Property }\end{array}$ \\
\hline Muqami Tehrik-e-Taliban (MTT) & Nationalist & 2008 & - & Afghanistan, Pakistan & Armed Assault & Military \\
\hline Muslim Mujahideen & Religious & 1996 & - & India, Pakistan & Unarmed Assault & Government (General) \\
\hline Muslim United Army & Religious & 2002 & 2003 & Pakistan & Bombing/Explosion & Businesses, Police \\
\hline $\begin{array}{l}\text { Muslim United Liberation Front of } \\
\text { Assam (MULFA) }\end{array}$ & Religious & 1996 & - & Bangladesh & $\begin{array}{l}\text { Armed Assault, Bombing/Explosion, } \\
\text { Hostage Taking }\end{array}$ & $\begin{array}{l}\text { Businesses, Government (General), } \\
\text { Police, Private Citizens \& Property }\end{array}$ \\
\hline $\begin{array}{l}\text { Muslim United Liberation Tigers of } \\
\text { Assam (MULTA) }\end{array}$ & Nationalist & 1996 & - & India, Pakistan & Bombing/Explosion, Hostage Taking & Unknown \\
\hline Muslims Against Global Oppression & Religious & 1998 & 1998 & South Africa & Bombing/Explosion & Businesses, Private Citizens \& Property \\
\hline Mutahida Majlis-e-Amal (MMA) & Religious & 2006 & 2006 & India, Pakistan & Armed Assault, Bombing/Explosion & Police, Transportation \\
\hline Nadim al-Maghrebi & Religious & 2006 & - & Algeria & Armed Assault, Bombing/Explosion & Government (General) \\
\hline Naga National Council (NNC) & Nationalist & 2011 & - & India & Bombing/Explosion, Hostage Taking & $\begin{array}{l}\text { Military, Terrorists/Non-State Militia, } \\
\text { Transportation }\end{array}$ \\
\hline Naga People's Council (NPC) & Nationalist & 2008 & - & India & Bombing/Explosion & Unknown \\
\hline Naga Students Federation & Nationalist & 1994 & 1994 & India & Bombing/Explosion & Businesses, Utilities \\
\hline $\begin{array}{l}\text { Nagorno-Karabakh Republic Defense } \\
\text { Army/Artsakh Republic Defense Army }\end{array}$ & Nationalist & 1992 & - & Armenia & $\begin{array}{l}\text { Armed Assault, Bombing/Explosion, } \\
\text { Facility/Infrastructure Attack }\end{array}$ & Military \\
\hline $\begin{array}{l}\text { National Accord Movement/Iraqi } \\
\text { National Accord (INA) }\end{array}$ & Nationalist & 1995 & 1995 & Iraq, Jordan & Bombing/Explosion & Government (General) \\
\hline National Alliance - Chad & Nationalist & 2008 & - & Chad & Armed Assault & Military, Private Citizens \& Property \\
\hline
\end{tabular}




\begin{tabular}{|c|c|c|c|c|c|c|}
\hline VEO Name & Type & Emerged & Ended & Countries & Type of Attack & Target Type \\
\hline National Anti-Corruption Front & Left-Wing & 2005 & 2005 & Bolivia & Bombing/Explosion & Businesses, Utilities \\
\hline National Bolshevik Party (PNB) & Left-Wing & 2000 & - & Latvia & $\begin{array}{l}\text { Assassination, Bombing/Explosion, } \\
\text { Facility/Infrastructure Attack }\end{array}$ & $\begin{array}{l}\text { Government (General), Religious } \\
\text { Figures/Institutions }\end{array}$ \\
\hline $\begin{array}{l}\text { National Coalition of Syrian } \\
\text { Revolutionary and Opposition Forces }\end{array}$ & Nationalist & 2012 & - & Syria & Unknown & Government (General), Military \\
\hline $\begin{array}{l}\text { National Committee for the Restoration } \\
\text { of Democracy and State (CNRDR) }\end{array}$ & Nationalist & 2012 & - & Mali & Armed Assault & Government (General) \\
\hline $\begin{array}{l}\text { National Congress for the Defense of the } \\
\text { People (CNDP) }\end{array}$ & $\begin{array}{l}\text { Right- } \\
\text { Wing }\end{array}$ & 2006 & - & Congo, Democratic Republic & Armed Assault, Bombing/Explosion & $\begin{array}{l}\text { Government (Diplomatic), NGO, Private } \\
\text { Citizens \& Property, Terrorists/Non- } \\
\text { State Militia }\end{array}$ \\
\hline $\begin{array}{l}\text { National Council for Defense of } \\
\text { Democracy (NCDD) }\end{array}$ & Nationalist & 1995 & 2004 & Burundi & $\begin{array}{l}\text { Armed Assault, Bombing/Explosion, } \\
\text { Facility/Infrastructure Attack, } \\
\text { Hijacking, Hostage Taking }\end{array}$ & $\begin{array}{l}\text { Businesses, Educational Institutes, } \\
\text { Government (Diplomatic), Maritime, } \\
\text { Military, Police, Private Citizens \& } \\
\text { Property, Transportation, Utilities }\end{array}$ \\
\hline $\begin{array}{l}\text { National Council of the Union of Burma } \\
\text { (NCUB) }\end{array}$ & Nationalist & 1992 & - & Myanmar, Burma & Unarmed Assault & Government (General), Military \\
\hline National Defense Force (NDF) - Syria & Nationalist & 2012 & - & Syria & Armed Assault & Terrorists/Non-State Militia \\
\hline National Democratic Alliance (NDA) & Nationalist & 1995 & - & Uganda & $\begin{array}{l}\text { Armed Assault, Assassination, Hostage } \\
\text { Taking }\end{array}$ & Government (General), Police \\
\hline National Democratic Alliance of Sudan & Nationalist & 1999 & 1999 & Sudan & Armed Assault & Transportation \\
\hline National Democratic Front of Liberia & Nationalist & 1992 & 1992 & Liberia & Armed Assault & Religious Figures/Institutions \\
\hline $\begin{array}{l}\text { National Democratic Party of } \\
\text { Bangladesh }\end{array}$ & Nationalist & 1994 & 1994 & Bangladesh, Cuba & $\begin{array}{l}\text { Armed Assault, Assassination, } \\
\text { Facility/Infrastructure Attack }\end{array}$ & Government (General), Utilities \\
\hline $\begin{array}{l}\text { National Development Party (NDP) - } \\
\text { Kenya }\end{array}$ & Nationalist & 1997 & 1997 & Kenya & Armed Assault & Government (General) \\
\hline National Front Against Tigers (NFAT) & Nationalist & 2000 & 2001 & Sri Lanka & Bombing/Explosion & Government (Diplomatic), NGO \\
\hline National Front for the Liberation of Iraq & Nationalist & 2004 & - & Iraq & Armed Assault, Bombing/Explosion & Government (General), Military \\
\hline National Front Security Service & $\begin{array}{l}\text { Right- } \\
\text { Wing }\end{array}$ & 1992 & 1992 & France & Assassination & Government (General) \\
\hline $\begin{array}{l}\text { National Ideological Resistance in Syria } \\
\text { (NIR }\end{array}$ & Nationalist & 2009 & - & Syria & Armed Assault & Government (General) \\
\hline National Liberation Alliance of Sa Kaeo & Nationalist & 2006 & 2006 & Thailand & Bombing/Explosion & Educational Institutes \\
\hline $\begin{array}{l}\text { National Liberation Army - Nepal } \\
\text { (GNFL) }\end{array}$ & Nationalist & 2008 & - & Nepal & Bombing/Explosion & Government (General) \\
\hline $\begin{array}{l}\text { National Liberation Army (NLA) - } \\
\text { Macedonia }\end{array}$ & Nationalist & 1999 & 2002 & Albania, Kosovo, Macedonia & $\begin{array}{l}\text { Armed Assault, Bombing/Explosion, } \\
\text { Facility/Infrastructure Attack, } \\
\text { Hijacking, Hostage Taking }\end{array}$ & $\begin{array}{l}\text { Businesses, Military, Police, Private } \\
\text { Citizens \& Property, Religious } \\
\text { Figures/Institutions, Transportation }\end{array}$ \\
\hline
\end{tabular}




\begin{tabular}{|c|c|c|c|c|c|c|}
\hline VEO Name & Type & Emerged & Ended & Countries & $\begin{array}{c}\text { Type of Attack } \\
\end{array}$ & $\begin{array}{c}\text { Target Type } \\
\end{array}$ \\
\hline $\begin{array}{l}\text { National Liberation Front - Burundi } \\
\text { (FLN) }\end{array}$ & Nationalist & 2008 & - & Burundi & $\begin{array}{l}\text { Armed Assault, Bombing/Explosion, } \\
\text { Hostage Taking }\end{array}$ & $\begin{array}{l}\text { Businesses, Educational Institutes, } \\
\text { Government (Diplomatic), Government } \\
\text { (General), Military, Private Citizens \& } \\
\text { Property, Religious Figures/Institutions, } \\
\text { Transportation }\end{array}$ \\
\hline National Militia of Tripura & Nationalist & 2000 & - & Indonesia & Bombing/Explosion, Unarmed Assault & Unknown \\
\hline $\begin{array}{l}\text { National Movement for the Liberation of } \\
\text { Azawad (MNLA)/Azawad National } \\
\text { Liberation Movement (MNLA) }\end{array}$ & Nationalist & 2011 & - & Mali & $\begin{array}{l}\text { Armed Assault, Bombing/Explosion, } \\
\text { Hijacking, Hostage Taking }\end{array}$ & $\begin{array}{l}\text { Government (Diplomatic), Government } \\
\text { (General), Journalists \& Media, Military, } \\
\text { Private Citizens \& Property }\end{array}$ \\
\hline $\begin{array}{l}\text { National Movement for the Restoration } \\
\text { of Pakistani Sovereignty (MNRPS) }\end{array}$ & Religious & 2002 & - & Pakistan & Hostage Taking & Journalists \& Media \\
\hline $\begin{array}{l}\text { National Movement of Revolutionaries } \\
\text { (MNR) }\end{array}$ & Nationalist & 2015 & - & Congo, Democratic Republic & Armed Assault, Bombing/Explosion & Government (General), Military \\
\hline $\begin{array}{l}\text { National People's Movement } \\
\text { Coordination Committee }\end{array}$ & Left-Wing & 1996 & 1996 & Nepal & Armed Assault & Private Citizens \& Property \\
\hline National People's Party (RJD) & Nationalist & 1997 & 2000 & India & Armed Assault, Unarmed Assault & Government (General) \\
\hline National Redemption Front & Nationalist & 2006 & 2006 & Sudan & Armed Assault & Utilities \\
\hline National Socialist Initiative & $\begin{array}{l}\text { Right- } \\
\text { Wing }\end{array}$ & 2009 & - & Russia & Unarmed Assault & Unknown \\
\hline $\begin{array}{l}\text { National Socialist Movement United } \\
\text { Kingdom (NSM) }\end{array}$ & $\begin{array}{l}\text { Right- } \\
\text { Wing }\end{array}$ & 1997 & 1999 & United Kingdom & Bombing/Explosion & Private Citizens \& Property \\
\hline National Socialist Underground (NSU) & $\begin{array}{l}\text { Right- } \\
\text { Wing }\end{array}$ & 1996 & - & Germany & Armed Assault, Bombing/Explosion & $\begin{array}{l}\text { Businesses, Police, Private Citizens \& } \\
\text { Property, Transportation }\end{array}$ \\
\hline National Union - Israel & $\begin{array}{l}\text { Right- } \\
\text { Wing }\end{array}$ & 1999 & - & Israel, Palestinian State & Armed Assault & Military, Private Citizens \& Property \\
\hline National Warriors & $\begin{array}{l}\text { Right- } \\
\text { Wing }\end{array}$ & 2002 & 2002 & South Africa & Bombing/Explosion & Private Citizens \& Property \\
\hline National Youth Service of Zimbabwe & Left-Wing & 2002 & - & Zimbabwe & Unarmed Assault & $\begin{array}{l}\text { Government (General), Private Citizens } \\
\text { \& Property }\end{array}$ \\
\hline Nationalist Integrationist Front (FNI) & Nationalist & 2003 & 2005 & Congo, Democratic Republic & Armed Assault, Unarmed Assault & $\begin{array}{l}\text { Government (Diplomatic), Military, } \\
\text { NGO, Private Citizens \& Property }\end{array}$ \\
\hline Nationalist Kurdish Revenge Teams & Nationalist & 1999 & 1999 & Turkey & Bombing/Explosion & Government (General) \\
\hline National-Socialist Society (NSS) & $\begin{array}{l}\text { Right- } \\
\text { Wing }\end{array}$ & 2004 & - & Russia & Armed Assault & Private Citizens \& Property \\
\hline Nationalsocialistisk Front (NSF) & $\begin{array}{l}\text { Right- } \\
\text { Wing }\end{array}$ & 1994 & 1999 & Sweden & Bombing/Explosion & $\begin{array}{l}\text { Government (General), Private Citizens } \\
\text { \& Property }\end{array}$ \\
\hline Nationella Republikanska Armen (NRA) & $\begin{array}{l}\text { Right- } \\
\text { Wing } \\
\end{array}$ & 1998 & - & Sweden & Armed Assault & Businesses \\
\hline Nation's Army - Jerusalem & Religious & 2006 & - & Israel, Palestinian State & Armed Assault & Private Citizens \& Property \\
\hline
\end{tabular}




\begin{tabular}{|c|c|c|c|c|c|c|}
\hline VEO Name & Type & Emerged & Ended & Countries & Type of Attack & Target Type \\
\hline Nek Muhammad Group & Nationalist & 2001 & - & Afghanistan, Pakistan & Armed Assault & Military \\
\hline Neo Jamaah Islamiah & Religious & 2016 & - & Indonesia & Armed Assault & Unknown \\
\hline Neo-Nazis - Russia & $\begin{array}{l}\text { Right- } \\
\text { Wing }\end{array}$ & 1998 & - & $\begin{array}{l}\text { Austria, Belgium, Bosnia and } \\
\text { Herzegovina, Croatia, Estonia, France, } \\
\text { Germany, Mongolia, Russia, Serbia, } \\
\text { Sweden }\end{array}$ & $\begin{array}{l}\text { Armed Assault, Assassination, Unarmed } \\
\text { Assault }\end{array}$ & $\begin{array}{l}\text { Government (Diplomatic), Military, } \\
\text { Police, Private Citizens \& Property, } \\
\text { Religious Figures/Institutions }\end{array}$ \\
\hline Nepal Defense Army & Religious & 2008 & - & Nepal & Bombing/Explosion & $\begin{array}{l}\text { Government (General), Journalists \& } \\
\text { Media, Religious Figures/Institutions }\end{array}$ \\
\hline Nepal People's Army & Left-Wing & 2001 & - & Nepal & Bombing/Explosion & Government (General) \\
\hline New Patriotic Party (NPP)- Ghana & $\begin{array}{l}\text { Right- } \\
\text { Wing }\end{array}$ & 1992 & 1996 & Ghana & Armed Assault, Unarmed Assault & $\begin{array}{l}\text { Government (General), Private Citizens } \\
\text { \& Property }\end{array}$ \\
\hline $\begin{array}{l}\text { New Pattani United Liberation } \\
\text { Organization (PULO) }\end{array}$ & Nationalist & 1995 & - & Malaysia, Thailand & Hostage Taking & $\begin{array}{l}\text { Educational Institutes, Government } \\
\text { (General), Police }\end{array}$ \\
\hline New Revolutionary Alternative (NRA) & Left-Wing & 1999 & 1999 & Russia & Bombing/Explosion & Police \\
\hline $\begin{array}{l}\text { New Revolutionary Popular Struggle } \\
\text { (NELA) }\end{array}$ & Left-Wing & 2002 & 2002 & Greece & Bombing/Explosion & Maritime \\
\hline Niger Delta Avengers (NDA) & Nationalist & 2016 & - & Nigeria & Armed Assault, Bombing/Explosion & Businesses, Utilities \\
\hline Niger Delta Freedom Fighters & Nationalist & 2008 & - & Nigeria & Bombing/Explosion & Utilities \\
\hline $\begin{array}{l}\text { Niger Delta Greenland Justice Mandate } \\
\text { (NDGJM) }\end{array}$ & Nationalist & 2016 & - & Nigeria & $\begin{array}{l}\text { Bombing/Explosion, } \\
\text { Facility/Infrastructure Attack }\end{array}$ & Utilities \\
\hline $\begin{array}{l}\text { Niger Delta Patriotic Force/Niger Delta } \\
\text { Vigilante (NDV) }\end{array}$ & Nationalist & 2003 & - & Niger, Nigeria & $\begin{array}{l}\text { Armed Assault, Bombing/Explosion, } \\
\text { Hostage Taking }\end{array}$ & Businesses, Maritime, Police, Utilities \\
\hline $\begin{array}{l}\text { Niger Delta People's Volunteer Force } \\
\text { (NDPVF) }\end{array}$ & Nationalist & 2004 & - & Nigeria & $\begin{array}{l}\text { Bombing/Explosion, } \\
\text { Facility/Infrastructure Attack, Hostage } \\
\text { Taking }\end{array}$ & Private Citizens \& Property, Utilities \\
\hline $\begin{array}{l}\text { Niger Delta Revolutionary Crusaders } \\
\text { (NDRC) }\end{array}$ & Nationalist & 2016 & & Nigeria & Armed Assault, Bombing/Explosion & Terrorists/Non-State Militia \\
\hline Niger Movement for Justice (MNJ) & Nationalist & 2008 & & Chad, Libya, Mali, Niger & Armed Assault, Hostage Taking & $\begin{array}{l}\text { Airports \& Aircraft, Government } \\
\text { (General), Military, Utilities }\end{array}$ \\
\hline Night Avengers & $\begin{array}{l}\text { Right- } \\
\text { Wing }\end{array}$ & 1997 & 1998 & Honduras & Assassination & Government (General) \\
\hline Nihilist Faction & Left-Wing & 1996 & 1996 & Greece & $\begin{array}{l}\text { Bombing/Explosion, } \\
\text { Facility/Infrastructure Attack }\end{array}$ & $\begin{array}{l}\text { Businesses, Government (General), } \\
\text { Journalists \& Media, Police, Private } \\
\text { Citizens \& Property, Religious } \\
\text { Figures/Institutions }\end{array}$ \\
\hline $\begin{array}{l}\text { Ninawa Mujahideen in the city of } \\
\text { Mosul/Mujahideen of Nineveh }\end{array}$ & Religious & 2005 & 2005 & Iraq & Hostage Taking & Government (General) \\
\hline
\end{tabular}




\begin{tabular}{|c|c|c|c|c|c|c|}
\hline VEO Name & Type & Emerged & Ended & Countries & Type of Attack & Target Type \\
\hline Ninjas Malitia & Nationalist & 1993 & - & Congo, Democratic Republic, Indonesia & $\begin{array}{l}\text { Armed Assault, Bombing/Explosion, } \\
\text { Hostage Taking, Unarmed Assault }\end{array}$ & $\begin{array}{l}\text { Military, NGO, Police, Private Citizens \& } \\
\text { Property, Transportation }\end{array}$ \\
\hline Nograi Jamaat & Religious & 2016 & - & Syria & $\begin{array}{l}\text { Armed Assault, Assassination, } \\
\text { Bombing/Explosion }\end{array}$ & $\begin{array}{l}\text { Government (General), Military, Private } \\
\text { Citizens \& Property, Terrorists/Non- } \\
\text { State Militia }\end{array}$ \\
\hline $\begin{array}{l}\text { Nordiska Motstandsrorelsen } \\
\text { (SMR)/Nordic Resistance Movement }\end{array}$ & $\begin{array}{l}\text { Right- } \\
\text { Wing }\end{array}$ & 1997 & - & Sweden & Armed Assault, Unarmed Assault & Private Citizens \& Property \\
\hline $\begin{array}{l}\text { Northern Alliance/United Islamic Front } \\
\text { for the Salvation of Afghanistan (UIFSA) }\end{array}$ & Nationalist & 1999 & 2001 & Afghanistan & Bombing/Explosion & $\begin{array}{l}\text { Government (General), Police, } \\
\text { Terrorists/Non-State Militia }\end{array}$ \\
\hline November 25 Anarchist Group & Left-Wing & 1996 & 1996 & Greece & Bombing/Explosion & Businesses \\
\hline Nuclei Communist Combatants & Left-Wing & 1993 & 1994 & Italy & Bombing/Explosion & Military \\
\hline Nuclei for Promoting Total Catastrophe & Left-Wing & 2003 & 2003 & Greece & Bombing/Explosion & Government (General) \\
\hline $\begin{array}{l}\text { Nucleus of Conspirators for the } \\
\text { Extension of Chaos }\end{array}$ & Left-Wing & 2011 & - & Argentina & Bombing/Explosion & Government (General) \\
\hline Nucleus of Fighting Communists & Left-Wing & 1992 & 1992 & Italy & Bombing/Explosion & Private Citizens \& Property \\
\hline Nur-al-Din al-Zinki Movement & Religious & 2016 & - & Syria & Bombing/Explosion & $\begin{array}{l}\text { Government (General), Military, Private } \\
\text { Citizens \& Property }\end{array}$ \\
\hline Nusantara Islamic Jihad Forces & Religious & 1999 & 1999 & Indonesia & Armed Assault & Police, Religious Figures/Institutions \\
\hline NVF (Narodnaya Volya) & Nationalist & 2009 & - & Russia & Armed Assault, Bombing/Explosion & Police, Private Citizens \& Property \\
\hline Odua People's Congress (OPC) & Left-Wing & 1995 & - & Nigeria & $\begin{array}{l}\text { Armed Assault, Assassination, } \\
\text { Bombing/Explosion, } \\
\text { Facility/Infrastructure Attack, Hostage } \\
\text { Taking, Unarmed Assault }\end{array}$ & Government (General), Military, Police \\
\hline Oglaigh na hEireann & Nationalist & 2009 & - & Ireland & $\begin{array}{l}\text { Armed Assault, Bombing/Explosion, } \\
\text { Hijacking, Hostage Taking }\end{array}$ & $\begin{array}{l}\text { Businesses, Military, Police, Private } \\
\text { Citizens \& Property, Transportation }\end{array}$ \\
\hline Okba Ibn Nafaa Brigade & Religious & 2014 & - & Algeria, Tunisia & $\begin{array}{l}\text { Armed Assault, Bombing/Explosion, } \\
\text { Hostage Taking }\end{array}$ & $\begin{array}{l}\text { Military, Police, Private Citizens \& } \\
\text { Property }\end{array}$ \\
\hline Old School Society (OSS) & $\begin{array}{l}\text { Right- } \\
\text { Wing }\end{array}$ & 2014 & - & Germany & Bombing/Explosion & Private Citizens \& Property \\
\hline Olga Nucleus & Left-Wing & 2012 & - & Italy, Switzerland & $\begin{array}{l}\text { Armed Assault, Bombing/Explosion, } \\
\text { Hostage Taking }\end{array}$ & $\begin{array}{l}\text { Businesses, Government (Diplomatic), } \\
\text { Government (General) }\end{array}$ \\
\hline Omar bin al-Khattab Brigades (KOK) & Nationalist & 2005 & 2005 & Palestinian State & Hostage Taking & Government (Diplomatic) \\
\hline Omer Khalid Group & Religious & 2007 & - & Pakistan & $\begin{array}{l}\text { Armed Assault, Assassination, } \\
\text { Bombing/Explosion, Hostage Taking }\end{array}$ & Military, Religious Figures/Institutions \\
\hline $\begin{array}{l}\text { Opposition Jatiya Samajtantrik Del } \\
\text { Party }\end{array}$ & Left-Wing & 1994 & 1994 & Bangladesh & Unarmed Assault & Police, Private Citizens \& Property \\
\hline
\end{tabular}




\begin{tabular}{|c|c|c|c|c|c|c|}
\hline VEO Name & Type & Emerged & Ended & Countries & Type of Attack & Target Type \\
\hline Orange Volunteers & Nationalist & 1998 & 2001 & Ireland, United Kingdom & $\begin{array}{l}\text { Armed Assault, Bombing/Explosion, } \\
\text { Facility/Infrastructure Attack }\end{array}$ & $\begin{array}{l}\text { Businesses, Private Citizens \& Property, } \\
\text { Religious Figures/Institutions, } \\
\text { Terrorists/Non-State Militia }\end{array}$ \\
\hline Order 777 & $\begin{array}{l}\text { Right- } \\
\text { Wing }\end{array}$ & 2006 & - & Liberia, Serbia, United Kingdom & Armed Assault, Assassination & Government (General) \\
\hline Organization for National Will - Mexico & Nationalist & 2007 & - & Mexico & Unknown & Businesses, Tourists \\
\hline $\begin{array}{l}\text { Organization of Free Young Algerians } \\
\text { (OJAL) }\end{array}$ & Nationalist & 1992 & 1996 & Algeria & $\begin{array}{l}\text { Armed Assault, Assassination, Unarmed } \\
\text { Assault }\end{array}$ & Private Citizens \& Property \\
\hline Overthrown Anarchist Faction & Left-Wing & 2000 & 2000 & Greece & Bombing/Explosion & Government (Diplomatic) \\
\hline Pacific Popular Front & Left-Wing & 1995 & 1995 & Australia & $\begin{array}{l}\text { Facility/Infrastructure Attack, Hostage } \\
\text { Taking }\end{array}$ & Government (Diplomatic) \\
\hline Padanian Armed Separatist Phalanx & Nationalist & 1998 & 1998 & Italy & Armed Assault & Government (General), Police \\
\hline Palestinian Islamic Army (PIA) & Religious & 2008 & - & Egypt & Bombing/Explosion & $\begin{array}{l}\text { Businesses, Religious } \\
\text { Figures/Institutions, Tourists }\end{array}$ \\
\hline Palestinian Islamic Revolutionary Army & Religious & 1993 & - & Cuba, Lebanon, Palestinian State & Bombing/Explosion & Military \\
\hline Panama Defense Force & Nationalist & 1992 & 1992 & Netherlands, Panama & Bombing/Explosion & $\begin{array}{l}\text { Airports \& Aircraft, Government } \\
\text { (General) }\end{array}$ \\
\hline $\begin{array}{l}\text { Pan-Caucasian Mujahideen/Doka } \\
\text { Umarov's Group }\end{array}$ & Religious & 2006 & - & Russia & Bombing/Explosion & Airports \& Aircraft, Transportation \\
\hline Paraguayan People's Army (EPP) & Left-Wing & 2008 & - & Paraguay & $\begin{array}{l}\text { Armed Assault, Assassination, } \\
\text { Facility/Infrastructure Attack, Hostage } \\
\text { Taking }\end{array}$ & $\begin{array}{l}\text { Businesses, Government (General), } \\
\text { Journalists \& Media, Military, Police, } \\
\text { Private Citizens \& Property, Utilities }\end{array}$ \\
\hline Partai Aceh & Nationalist & 2009 & - & Indonesia, Malaysia & Assassination, Bombing/Explosion & Government (General) \\
\hline Partai Bulan Bintang & Religious & 1998 & - & Indonesia & Unknown & Unknown \\
\hline Partisians of the Sunni & Religious & 2005 & - & Iraq & Armed Assault & Religious Figures/Institutions \\
\hline $\begin{array}{l}\text { Party for Unity and Safeguarding of the } \\
\text { Integrity of Congo (PUSIC) }\end{array}$ & Nationalist & 2003 & - & Congo, Democratic Republic, Uganda & $\begin{array}{l}\text { Armed Assault, Bombing/Explosion, } \\
\text { Hostage Taking, Unarmed Assault }\end{array}$ & $\begin{array}{l}\text { Government (General), Terrorists/Non- } \\
\text { State Militia, Violent Political Party }\end{array}$ \\
\hline Party of Free Life of Kurdistan (PJAK) & Nationalist & 2006 & - & Iraq, Syria, Turkey & Armed Assault & Military, Police \\
\hline Pasban-i-Islam & Religious & 2004 & - & Pakistan & Bombing/Explosion & Religious Figures/Institutions \\
\hline $\begin{array}{l}\text { Patriotic Europeans Against the Islamic } \\
\text { of the West (PEGIDA) }\end{array}$ & $\begin{array}{l}\text { Right- } \\
\text { Wing }\end{array}$ & 2014 & - & Germany & Bombing/Explosion & Private Citizens \& Property \\
\hline $\begin{array}{l}\text { Patriotic Movement of Cote d'Ivoire } \\
\text { (MCPI) }\end{array}$ & Nationalist & 2002 & - & Cote D'Ivoire & Armed Assault, Bombing/Explosion & Government (General), Military \\
\hline $\begin{array}{l}\text { Patriotic Resistance Front in Ituri } \\
\text { (FRPI) }\end{array}$ & Nationalist & 2002 & - & Congo, Democratic Republic & $\begin{array}{l}\text { Armed Assault, Assassination, } \\
\text { Facility/Infrastructure Attack, Hostage } \\
\text { Taking }\end{array}$ & $\begin{array}{l}\text { Government (Diplomatic), Government } \\
\text { (General), Military, NGO, Private } \\
\text { Citizens \& Property }\end{array}$ \\
\hline
\end{tabular}




\begin{tabular}{|c|c|c|c|c|c|c|}
\hline VEO Name & Type & Emerged & Ended & Countries & Type of Attack & Target Type \\
\hline $\begin{array}{l}\text { Peasant Self-Defense Group } \\
\text { (ACCU)/Self-Defense Groups of } \\
\text { Cordoba and Uraba }\end{array}$ & $\begin{array}{l}\text { Right- } \\
\text { Wing }\end{array}$ & 1994 & 2006 & Colombia & Armed Assault, Hostage Taking & $\begin{array}{l}\text { Government (General), Police, Private } \\
\text { Citizens \& Property }\end{array}$ \\
\hline $\begin{array}{l}\text { People Against Gangsterism and Drugs } \\
\text { (PAGAD) }\end{array}$ & Religious & 1995 & - & South Africa & $\begin{array}{l}\text { Armed Assault, Assassination, } \\
\text { Bombing/Explosion }\end{array}$ & $\begin{array}{l}\text { Airports \& Aircraft, Businesses, } \\
\text { Government (General), Police, Private } \\
\text { Citizens \& Property, Religious } \\
\text { Figures/Institutions, Tourists }\end{array}$ \\
\hline People's Alliance & Religious & 1994 & 1996 & Sri Lanka & Armed Assault & $\begin{array}{l}\text { Government (General), Journalists \& } \\
\text { Media }\end{array}$ \\
\hline People's Army's Pioneers & Left-Wing & 2001 & 2001 & Israel & Bombing/Explosion & Police, Private Citizens \& Property \\
\hline People's Combatants' Group (GCP) & Left-Wing & 2000 & - & Ecuador & Assassination & $\begin{array}{l}\text { Government (General), Religious } \\
\text { Figures/Institutions }\end{array}$ \\
\hline People's Democratic Party (PDP) & $\begin{array}{l}\text { Right- } \\
\text { Wing }\end{array}$ & 2001 & - & Nigeria & $\begin{array}{l}\text { Assassination, Facility/Infrastructure } \\
\text { Attack, Hostage Taking }\end{array}$ & $\begin{array}{l}\text { Government (General), Private Citizens } \\
\text { \& Property }\end{array}$ \\
\hline People's Fatherland Movement & Nationalist & 1992 & 1993 & Chile & Armed Assault, Bombing/Explosion & $\begin{array}{l}\text { Businesses, Government (General), } \\
\text { Police, Utilities, Violent Political Party }\end{array}$ \\
\hline $\begin{array}{l}\text { People's Front for Democracy and } \\
\text { Justice (PFDJ) }\end{array}$ & Nationalist & 1993 & - & Eritrea & Unknown & Government (General) \\
\hline $\begin{array}{l}\text { People's Guerrilla Army (PGA)/People's } \\
\text { Liberation Guerrilla Army (PGLA) }\end{array}$ & Left-Wing & 2000 & - & India & Armed Assault & Government (General) \\
\hline People's Guerrilla Front & Left-Wing & 1992 & 1992 & Peru & Armed Assault, Assassination & Private Citizens \& Property \\
\hline $\begin{array}{l}\text { People's Liberation Army (PLA) - Sri } \\
\text { Lanka }\end{array}$ & Left-Wing & 2009 & - & Sri Lanka & Armed Assault & Government (General), Military \\
\hline People's Liberation Forces & Left-Wing & 1998 & - & Colombia & Armed Assault, Bombing/Explosion & Government (General) \\
\hline $\begin{array}{l}\text { People's Liberation Front of India } \\
\text { (PLFI) }\end{array}$ & Left-Wing & 2009 & - & India & $\begin{array}{l}\text { Armed Assault, Assassination, } \\
\text { Bombing/Explosion, } \\
\text { Facility/Infrastructure Attack, Hostage } \\
\text { Taking, Unarmed Assault }\end{array}$ & $\begin{array}{l}\text { Businesses, Government (General), } \\
\text { Police, Private Citizens \& Property, } \\
\text { Terrorists/Non-State Militia, } \\
\text { Transportation, Violent Political Party }\end{array}$ \\
\hline $\begin{array}{l}\text { People's Liberation Guerilla Army } \\
\text { (PLGA) }\end{array}$ & Left-Wing & 2009 & - & India & Armed Assault & Military \\
\hline People's Militia of Dagestan & Nationalist & 1997 & 2010 & Russia & Bombing/Explosion, Hostage Taking & $\begin{array}{l}\text { Journalists \& Media, Police, Private } \\
\text { Citizens \& Property }\end{array}$ \\
\hline $\begin{array}{l}\text { People's Militia of the Donetsk People's } \\
\text { Republic }\end{array}$ & Nationalist & 2014 & - & Ukraine & $\begin{array}{l}\text { Armed Assault, Bombing/Explosion, } \\
\text { Facility/Infrastructure Attack, Hostage } \\
\text { Taking }\end{array}$ & Government (General), Military \\
\hline $\begin{array}{l}\text { People's Militia of the Donetsk People's } \\
\text { Republic }\end{array}$ & Nationalist & 2014 & - & Ukraine & $\begin{array}{l}\text { Armed Assault, Facility/Infrastructure } \\
\text { Attack, Hostage Taking }\end{array}$ & Government (General), Military \\
\hline
\end{tabular}




\begin{tabular}{|c|c|c|c|c|c|c|}
\hline VEO Name & Type & Emerged & Ended & Countries & Type of Attack & Target Type \\
\hline People's Protection Units (YPG) & Nationalist & 2011 & - & Iraq, Syria, Turkey & $\begin{array}{l}\text { Armed Assault, Assassination, } \\
\text { Bombing/Explosion }\end{array}$ & Military, Terrorists/Non-State Militia \\
\hline People's Revolutionary Army - Colombia & Left-Wing & 1995 & 2005 & Colombia & Armed Assault, Hostage Taking & $\begin{array}{l}\text { Government (General), Maritime, } \\
\text { Private Citizens \& Property }\end{array}$ \\
\hline $\begin{array}{l}\text { People's Revolutionary Army-Mexico } \\
\text { (ERP-Mexico) }\end{array}$ & Nationalist & 1996 & - & Mexico & Armed Assault & Government (General), Utilities \\
\hline People's Revolutionary Militias & Left-Wing & 2002 & 2003 & Ecuador & Armed Assault, Bombing/Explosion & $\begin{array}{l}\text { Businesses, Government (Diplomatic), } \\
\text { Government (General) }\end{array}$ \\
\hline People's Revolutionary Organization & Left-Wing & 1992 & 1997 & Argentina & $\begin{array}{l}\text { Armed Assault, Assassination, } \\
\text { Bombing/Explosion, Unarmed Assault }\end{array}$ & $\begin{array}{l}\text { Businesses, Government (Diplomatic), } \\
\text { Government (General), Journalists \& } \\
\text { Media, Military, Police, Transportation }\end{array}$ \\
\hline $\begin{array}{l}\text { People's United Democratic Movement } \\
\text { (PUDEMO) }\end{array}$ & Nationalist & 2006 & 2006 & Swaziland & $\begin{array}{l}\text { Bombing/Explosion, } \\
\text { Facility/Infrastructure Attack }\end{array}$ & Educational Institutes \\
\hline People's United Liberation Front (PULF) & Religious & 1995 & - & India & $\begin{array}{l}\text { Armed Assault, Bombing/Explosion, } \\
\text { Hostage Taking }\end{array}$ & $\begin{array}{l}\text { Businesses, Government (General), } \\
\text { Military, Private Citizens \& Property }\end{array}$ \\
\hline Phalanx of Destruction & Religious & 2000 & - & Algeria & Armed Assault, Bombing/Explosion & $\begin{array}{l}\text { Government (General), Private Citizens } \\
\text { \& Property }\end{array}$ \\
\hline $\begin{array}{l}\text { Popular Army for the Restoration of } \\
\text { Democracy (APRD) }\end{array}$ & Nationalist & 2006 & - & The Central African Republic & Armed Assault & $\begin{array}{l}\text { Military, NGO, Private Citizens \& } \\
\text { Property }\end{array}$ \\
\hline $\begin{array}{l}\text { Popular Army Vanguards-Battalions of } \\
\text { Return }\end{array}$ & Religious & 2001 & 2001 & Israel, Palestinian State & Armed Assault, Bombing/Explosion & Police, Private Citizens \& Property \\
\hline Popular Democratic Movement (MPD) & Left-Wing & 1992 & 1992 & Ecuador & Unknown & Government (General) \\
\hline Popular Fighters Group & Left-Wing & 2013 & - & Greece & Armed Assault, Bombing/Explosion & Businesses, Government (General) \\
\hline $\begin{array}{l}\text { Popular Front for Justice in the Congo } \\
\text { (FPJC) }\end{array}$ & Left-Wing & 2008 & - & Congo, Democratic Republic & Hostage Taking & $\begin{array}{l}\text { Government (General), Private Citizens } \\
\text { \& Property }\end{array}$ \\
\hline Popular Front for Recovery (FPR) & Nationalist & 2008 & & Central African Republic, Chad & Armed Assault, Hostage Taking & $\begin{array}{l}\text { Government (General), Military, Private } \\
\text { Citizens \& Property }\end{array}$ \\
\hline $\begin{array}{l}\text { Popular Front for the Liberation of the } \\
\text { Sahara }\end{array}$ & Nationalist & 1994 & 1994 & Niger & Armed Assault & Military \\
\hline Popular Justice - Greece & Left-Wing & 2003 & 2003 & Greece & Bombing/Explosion & Government (General) \\
\hline $\begin{array}{l}\text { Popular Resistance - Greece/Laiki } \\
\text { Antistasi }\end{array}$ & Left-Wing & 2000 & 2002 & Greece & Assassination, Bombing/Explosion & Businesses, Government (General) \\
\hline Popular Resistance Committees & Nationalist & 2000 & - & Israel, Palestinian State & $\begin{array}{l}\text { Armed Assault, Bombing/Explosion, } \\
\text { Hostage Taking }\end{array}$ & $\begin{array}{l}\text { Businesses, Educational Institutes, } \\
\text { Government (Diplomatic), Military, } \\
\text { Police, Private Citizens \& Property, } \\
\text { Religious Figures/Institutions, } \\
\text { Transportation }\end{array}$ \\
\hline
\end{tabular}




\begin{tabular}{|c|c|c|c|c|c|c|}
\hline VEO Name & Type & Emerged & Ended & Countries & Type of Attack & Target Type \\
\hline Popular Resistance Committees & Nationalist & 2015 & - & Saudi Arabia, Yemen & $\begin{array}{l}\text { Armed Assault, Assassination, } \\
\text { Bombing/Explosion, Hostage Taking }\end{array}$ & $\begin{array}{l}\text { Military, Police, Private Citizens \& } \\
\text { Property, Violent Political Party }\end{array}$ \\
\hline Popular Resistance Movement & Nationalist & 2014 & - & Egypt & $\begin{array}{l}\text { Armed Assault, Assassination, } \\
\text { Bombing/Explosion, } \\
\text { Facility/Infrastructure Attack }\end{array}$ & $\begin{array}{l}\text { Airports \& Aircraft, Businesses, } \\
\text { Government (General), Police }\end{array}$ \\
\hline Popular Revolutionary Action & Left-Wing & 2003 & 2005 & Greece & Bombing/Explosion & Businesses \\
\hline $\begin{array}{l}\text { Popular Revolutionary Army - Mexico } \\
\text { (EPR) }\end{array}$ & Left-Wing & 1996 & 2007 & Mexico & $\begin{array}{l}\text { Armed Assault, Bombing/Explosion, } \\
\text { Facility/Infrastructure Attack, Hostage } \\
\text { Taking }\end{array}$ & $\begin{array}{l}\text { Businesses, Journalists \& Media, } \\
\text { Military, Police, Private Citizens \& } \\
\text { Property, Utilities }\end{array}$ \\
\hline $\begin{array}{l}\text { Popular Revolutionary Front - Greece } \\
\text { (LEM) }\end{array}$ & Left-Wing & 1998 & 1998 & Greece & Bombing/Explosion & Government (Diplomatic) \\
\hline Popular Self-Defense Forces & Nationalist & 1993 & - & Congo, Democratic Republic & Armed Assault & $\begin{array}{l}\text { Businesses, Government (General), } \\
\text { Military, Private Citizens \& Property }\end{array}$ \\
\hline Popular Will & Left-Wing & 2004 & - & Greece & Bombing/Explosion & Businesses, Government (General) \\
\hline Porattom & Left-Wing & 2002 & 2002 & India & Facility/Infrastructure Attack & Businesses \\
\hline $\begin{array}{l}\text { Poso Terrorist Operations/Bandung } \\
\text { Terrorists }\end{array}$ & Religious & 2013 & - & Indonesia, Philippines & Armed Assault, Bombing/Explosion & $\begin{array}{l}\text { Government (Diplomatic), Police, } \\
\text { Tourists }\end{array}$ \\
\hline Praveen Dalam & Left-Wing & 2003 & 2003 & India & Assassination & Government (General) \\
\hline Proletarian Assault Group & Left-Wing & 2014 & - & Greece & Facility/Infrastructure Attack & Government (General) \\
\hline Proletarian Nuclei for Communism & Left-Wing & 2003 & 2004 & Italy & Bombing/Explosion & $\begin{array}{l}\text { Government (General), Private Citizens } \\
\text { \& Property }\end{array}$ \\
\hline Proletarian Reprisals - Greece & Left-Wing & 2002 & 2002 & Greece & Bombing/Explosion & Government (General) \\
\hline Pro-Russia Militia & Nationalist & 2014 & - & Ukraine & $\begin{array}{l}\text { Armed Assault, Assassination, } \\
\text { Bombing/Explosion, } \\
\text { Facility/Infrastructure Attack, Hostage } \\
\text { Taking, Unarmed Assault }\end{array}$ & $\begin{array}{l}\text { Government (General), Military, Police, } \\
\text { Private Citizens \& Property }\end{array}$ \\
\hline Pro-State Militia & Nationalist & 1995 & 1995 & Turkey & Armed Assault & Government (General) \\
\hline Protectors of Islam Brigade & Religious & 2005 & 2005 & Iraq & Hostage Taking & Journalists \& Media \\
\hline Pueblo Reagrupado & Left-Wing & 2002 & 2002 & Chile & Bombing/Explosion & Food or Water Supply \\
\hline Puka Inti Maoist Communist Party & Left-Wing & 1994 & 1994 & Ecuador & Bombing/Explosion, Hostage Taking & Businesses, Food or Water Supply \\
\hline $\begin{array}{l}\text { Punishment Brigade for the al-Jaafari } \\
\text { Government }\end{array}$ & Nationalist & 2005 & - & Iraq & Hostage Taking & Government (General) \\
\hline Punitive Left-Wing Front & Left-Wing & 1992 & 1992 & Nicaragua & Assassination, Bombing/Explosion & $\begin{array}{l}\text { Businesses, NGO, Private Citizens \& } \\
\text { Property, Transportation }\end{array}$ \\
\hline $\begin{array}{l}\text { Punjabi Taliban/Tehrik-i-Taliban- } \\
\text { Punjab }\end{array}$ & Religious & 2007 & - & Afghanistan, Pakistan & $\begin{array}{l}\text { Armed Assault, Assassination, Hostage } \\
\text { Taking }\end{array}$ & $\begin{array}{l}\text { Businesses, Private Citizens \& Property, } \\
\text { Religious Figures/Institutions }\end{array}$ \\
\hline
\end{tabular}




\begin{tabular}{|c|c|c|c|c|c|c|}
\hline VEO Name & Type & Emerged & Ended & Countries & Type of Attack & Target Type \\
\hline Purbo Banglar Communist Party & Left-Wing & 2002 & - & Bangladesh & $\begin{array}{l}\text { Armed Assault, Assassination, } \\
\text { Bombing/Explosion, Hostage Taking }\end{array}$ & $\begin{array}{l}\text { Businesses, Government (General), } \\
\text { Military, Private Citizens \& Property, } \\
\text { Terrorists/Non-State Militia, Violent } \\
\text { Political Party }\end{array}$ \\
\hline Pusat Persatuan Tadika Narathiwat & Religious & 1994 & - & Thailand & Armed Assault, Bombing/Explosion & $\begin{array}{l}\text { Government (General), Military, Private } \\
\text { Citizens \& Property }\end{array}$ \\
\hline Quetta Shura Taliban (QST) & Religious & 2001 & - & Pakistan & Armed Assault, Bombing/Explosion & Military \\
\hline Quwat Sahl Ninawa (QSN) & Religious & 2014 & - & Iraq & Unknown & Terrorists/Non-State Militia \\
\hline Rabha National Security Force (RNSF) & Nationalist & 2001 & - & India & Hostage Taking & Educational Institutes \\
\hline Racial Volunteer Force (RVF) & Nationalist & 2002 & - & United Kingdom & Bombing/Explosion & $\begin{array}{l}\text { Journalists \& Media, Private Citizens \& } \\
\text { Property, Violent Political Party }\end{array}$ \\
\hline Rafallah al-Sahati Brigade & Nationalist & 2012 & - & Libya & Armed Assault, Bombing/Explosion & Military, Private Citizens \& Property \\
\hline Raia Mutomboki & Nationalist & 2005 & - & Congo, Democratic Republic & Armed Assault, Hostage Taking & Military, Private Citizens \& Property \\
\hline Rajah Solaiman Islamic Movement & Religious & 2002 & - & Philippines & Bombing/Explosion & Maritime, Private Citizens \& Property \\
\hline Ramiro Ledesma Social Centre & $\begin{array}{l}\text { Right- } \\
\text { Wing }\end{array}$ & 2014 & - & Spain & Facility/Infrastructure Attack & $\begin{array}{l}\text { Government (General), Religious } \\
\text { Figures/Institutions }\end{array}$ \\
\hline Ranbir Sena & Nationalist & 1996 & - & India, Nepal & Armed Assault, Bombing/Explosion & $\begin{array}{l}\text { Airports \& Aircraft, Businesses, } \\
\text { Educational Institutes, Government } \\
\text { (General), Private Citizens \& Property, } \\
\text { Transportation, Violent Political Party }\end{array}$ \\
\hline Ras Kamboni Brigade & Religious & 2009 & - & Kenya, Somalia & Armed Assault, Assassination & $\begin{array}{l}\text { Private Citizens \& Property, } \\
\text { Terrorists/Non-State Militia }\end{array}$ \\
\hline Rashida Free Lions & Nationalist & 1999 & 2006 & Sudan & Armed Assault, Bombing/Explosion & Military \\
\hline Rawti Shax & Religious & 2009 & - & $\begin{array}{l}\text { Finland, Germany, Greece, Iran, Iraq, } \\
\text { Italy, Norway, Sweden, Switzerland, } \\
\text { Syria, United Kingdom }\end{array}$ & Bombing/Explosion, Hostage Taking & Government (Diplomatic) \\
\hline Real Irish Republican Army (RIRA) & Nationalist & 1998 & - & Ireland, United Kingdom & Armed Assault, Bombing/Explosion & $\begin{array}{l}\text { Government (General), Journalists \& } \\
\text { Media, Military, Private Citizens \& } \\
\text { Property }\end{array}$ \\
\hline Real Ulster Freedom Fighters (UFF) & $\begin{array}{l}\text { Right- } \\
\text { Wing }\end{array}$ & 2007 & - & Ireland, United Kingdom & Armed Assault, Bombing/Explosion & $\begin{array}{l}\text { Businesses, Military, Private Citizens \& } \\
\text { Property }\end{array}$ \\
\hline $\begin{array}{l}\text { Reassemblement Congolais Pour la } \\
\text { Democratic-Goma (RCD-G) }\end{array}$ & Nationalist & 1998 & 2003 & $\begin{array}{l}\text { Congo, Democratic Republic, Rwanda, } \\
\text { Uganda }\end{array}$ & Armed Assault, Hostage Taking & Government (General) \\
\hline $\begin{array}{l}\text { Reassemblement Congolais Pour la } \\
\text { Democratic-movement de Liberation } \\
\text { (RCD-ML) }\end{array}$ & Nationalist & 1998 & 2003 & Congo, Democratic Republic, Uganda & Armed Assault, Hostage Taking & Government (General) \\
\hline
\end{tabular}




\begin{tabular}{|c|c|c|c|c|c|c|}
\hline $\begin{array}{l}\text { VEO Name } \\
\end{array}$ & Type & Emerged & Ended & Countries & \begin{tabular}{|c|} 
Type of Attack \\
\end{tabular} & $\begin{array}{c}\text { Target Type } \\
\end{array}$ \\
\hline $\begin{array}{l}\text { Reassemblement Congolais Pour la } \\
\text { Democratic-Nationale (RCD-N) }\end{array}$ & Nationalist & 1998 & 2003 & $\begin{array}{l}\text { Belgium, Congo, Democratic Republic, } \\
\text { France }\end{array}$ & Armed Assault, Hostage Taking & Government (General), Military \\
\hline Rebolusyonaryong Hukbong Bayan & Left-Wing & 1998 & - & Philippines & Unknown & Government (General) \\
\hline Recontra 380 & $\begin{array}{l}\text { Right- } \\
\text { Wing } \\
\end{array}$ & 1993 & 1997 & Honduras, Nicaragua & Hostage Taking & Government (General) \\
\hline Red Commandos & Left-Wing & 1992 & 1992 & Italy, United Kingdom & Assassination & Private Citizens \& Property \\
\hline Red Daughters of Rage & Left-Wing & 1995 & 1995 & Austria & Bombing/Explosion & Businesses \\
\hline Red Egbesu Water Lions & Nationalist & 2016 & - & Nigeria & Bombing/Explosion & Utilities \\
\hline Red Guerrillas & Left-Wing & 2002 & 2002 & Russia & Bombing/Explosion & Government (General) \\
\hline Red Hand Defenders (RHD) & Nationalist & 1998 & - & Ireland, United Kingdom & $\begin{array}{l}\text { Armed Assault, Assassination, } \\
\text { Bombing/Explosion }\end{array}$ & $\begin{array}{l}\text { Businesses, Educational Institutes, } \\
\text { Government (General), Journalists \& } \\
\text { Media, Police, Private Citizens \& } \\
\text { Property, Terrorists/Non-State Militia, } \\
\text { Tourists }\end{array}$ \\
\hline Red Line & Left-Wing & 1999 & 1999 & Greece & Armed Assault & Government (Diplomatic), NGO \\
\hline Resistance Cell & Left-Wing & 2004 & 2004 & Greece & Bombing/Explosion & Businesses \\
\hline Resistenza Corsa & Nationalist & 2002 & 2003 & France & Armed Assault, Bombing/Explosion & Businesses, Private Citizens \& Property \\
\hline Revenge of the Hebrew Babies & Nationalist & 2002 & 2003 & Israel & Bombing/Explosion & Educational Institutes \\
\hline Revolutionary Action of Liberation & Left-Wing & 2006 & 2007 & Greece & $\begin{array}{l}\text { Bombing/Explosion, } \\
\text { Facility/Infrastructure Attack }\end{array}$ & Businesses, Government (General) \\
\hline $\begin{array}{l}\text { Revolutionary Armed Forces of the } \\
\text { People (FARP) }\end{array}$ & Left-Wing & 2000 & - & Mexico & $\begin{array}{l}\text { Armed Assault, Bombing/Explosion, } \\
\text { Hostage Taking }\end{array}$ & $\begin{array}{l}\text { Businesses, Government (General), } \\
\text { Military, Violent Political Party }\end{array}$ \\
\hline Revolutionary Army & Left-Wing & 2000 & - & Japan & Bombing/Explosion & Government (General) \\
\hline Revolutionary Brigades & Left-Wing & 2002 & 2002 & Greece & Bombing/Explosion & Government (General) \\
\hline $\begin{array}{l}\text { Revolutionary Communist Youth } \\
\text { League-Bolsheviks }\end{array}$ & Left-Wing & 1996 & - & Russia & Bombing/Explosion & Government (General) \\
\hline Revolutionary Force - Japan & Left-Wing & 2002 & 2002 & Japan & Bombing/Explosion & Military \\
\hline Revolutionary Front for Communism & Left-Wing & 1996 & - & Italy & Unknown & Businesses \\
\hline Revolutionary Leninist Brigades & Left-Wing & 2000 & 2000 & Italy & Bombing/Explosion & $\begin{array}{l}\text { Government (General), Violent Political } \\
\text { Party }\end{array}$ \\
\hline Revolutionary Liberation Action & Left-Wing & 2002 & - & Greece & $\begin{array}{l}\text { Bombing/Explosion, } \\
\text { Facility/Infrastructure Attack }\end{array}$ & Government (General) \\
\hline Revolutionary Memory & Left-Wing & 2003 & 2003 & Greece & Bombing/Explosion & Police \\
\hline $\begin{array}{l}\text { Revolutionary Movement for National } \\
\text { Salvation of South Sudan (REMNASA) }\end{array}$ & Nationalist & 2013 & - & Sudan & $\begin{array}{l}\text { Armed Assault, Facility/Infrastructure } \\
\text { Attack }\end{array}$ & Military, Transportation \\
\hline Revolutionary Nuclei & Left-Wing & 1996 & 2000 & Greece & Bombing/Explosion & Businesses, Government (General) \\
\hline Revolutionary Offensive Cells & Left-Wing & 2003 & - & Italy & Bombing/Explosion & Government (General) \\
\hline
\end{tabular}




\begin{tabular}{|c|c|c|c|c|c|c|}
\hline VEO Name & Type & Emerged & Ended & Countries & Type of Attack & Target Type \\
\hline Revolutionary Outburst Movement & Nationalist & 1993 & 1993 & Lebanon & Bombing/Explosion & Government (Diplomatic) \\
\hline Revolutionary Perspective & Left-Wing & 2000 & 2000 & Spain & Bombing/Explosion & Unknown \\
\hline Revolutionary Popular Left & Left-Wing & 1996 & 1996 & Greece & Armed Assault, Bombing/Explosion & $\begin{array}{l}\text { Businesses, Government (Diplomatic), } \\
\text { Government (General) }\end{array}$ \\
\hline Revolutionary Proletarian Army & Left-Wing & 1997 & 1997 & Philippines & Armed Assault & Police \\
\hline $\begin{array}{l}\text { Revolutionary Proletarian Initiative } \\
\text { Nuclei (NIPR) }\end{array}$ & Left-Wing & 2000 & - & Italy & Bombing/Explosion & NGO, Private Citizens \& Property \\
\hline Revolutionary Proletarian Nucleus & Left-Wing & 2000 & - & Italy & Bombing/Explosion & $\begin{array}{l}\text { Government (General), Private Citizens } \\
\text { \& Property }\end{array}$ \\
\hline Revolutionary Security Apparatus & Religious & 1992 & 1992 & Israel, Palestinian State & Assassination & Private Citizens \& Property \\
\hline Revolutionary Socialists & Left-Wing & 1999 & 2000 & Sweden & Unknown & Unknown \\
\hline Revolutionary Struggle & Left-Wing & 2003 & - & Greece & Bombing/Explosion & Government (General) \\
\hline Revolutionary Torch-Bearing Run & Left-Wing & 2004 & 2004 & Greece & Unknown & Unknown \\
\hline Revolutionary Violence Units (RVG) & Nationalist & 2001 & 2001 & Greece & Facility/Infrastructure Attack & Government (Diplomatic) \\
\hline $\begin{array}{l}\text { Revolutionary Workers Party - } \\
\text { Mindanao }\end{array}$ & Left-Wing & 2001 & - & Philippines & Unknown & Unknown \\
\hline Right Sector & Nationalist & 2013 & - & Ukraine & $\begin{array}{l}\text { Armed Assault, Bombing/Explosion, } \\
\text { Facility/Infrastructure Attack, Hostage } \\
\text { Taking }\end{array}$ & $\begin{array}{l}\text { Government (General), Journalists \& } \\
\text { Media, Police, Private Citizens \& } \\
\text { Property, Terrorists/Non-State Militia }\end{array}$ \\
\hline Riyad-us-Saliheyn Martyr's Brigade & Nationalist & 2002 & - & Russia & Bombing/Explosion & $\begin{array}{l}\text { Government (General), Private Citizens } \\
\text { \& Property }\end{array}$ \\
\hline Rizvon Sadirov Group & Religious & 1997 & 1997 & Tajikistan & Hostage Taking & $\begin{array}{l}\text { Government (Diplomatic), Private } \\
\text { Citizens \& Property }\end{array}$ \\
\hline Rohingya Mujahideen (RM) & Religious & 2013 & - & India, Myanmar, Burma, Sri Lanka & Bombing/Explosion & Religious Figures/Institutions \\
\hline Runda Kumpulan Kecil (RKK) & Nationalist & 2000 & - & Thailand & $\begin{array}{l}\text { Armed Assault, Bombing/Explosion, } \\
\text { Facility/Infrastructure Attack }\end{array}$ & $\begin{array}{l}\text { Businesses, Educational Institutes, } \\
\text { Government (General), Military, Police, } \\
\text { Private Citizens \& Property, } \\
\text { Transportation, Utilities }\end{array}$ \\
\hline Russian National Bolshevist Party & Left-Wing & 1993 & 2007 & $\begin{array}{l}\text { Estonia, Kyrgyzstan, Latvia, Moldova, } \\
\text { Russia }\end{array}$ & Bombing/Explosion & Religious Figures/Institutions \\
\hline Rwandan Patriotic Front (FPR) & Nationalist & 1992 & 1992 & Rwanda & $\begin{array}{l}\text { Armed Assault, Assassination, } \\
\text { Bombing/Explosion }\end{array}$ & $\begin{array}{l}\text { Businesses, Government (Diplomatic), } \\
\text { Government (General), Military, Private } \\
\text { Citizens \& Property, Religious } \\
\text { Figures/Institutions, Transportation }\end{array}$ \\
\hline Saad bin Abi Waqas Brigades & Religious & 2005 & - & Iraq & Armed Assault, Assassination & Government (General), Military \\
\hline Sabaot Land Defense Force (SLDF) & Nationalist & 2007 & - & Kenya & $\begin{array}{l}\text { Armed Assault, Assassination, } \\
\text { Bombing/Explosion }\end{array}$ & $\begin{array}{l}\text { Businesses, Private Citizens \& Property, } \\
\text { Religious Figures/Institutions }\end{array}$ \\
\hline
\end{tabular}




\begin{tabular}{|c|c|c|c|c|c|c|}
\hline VEO Name & Type & Emerged & Ended & Countries & Type of Attack & Target Type \\
\hline Sabiri Jamaat & Nationalist & 2013 & - & Russia, Syria, Tajikistan, Uzbekistan & Armed Assault, Bombing/Explosion & Military, Terrorists/Non-State Militia \\
\hline Sagrado Corazon Fanatic Group & Nationalist & 1997 & 1997 & Philippines & Unknown & Private Citizens \& Property \\
\hline $\begin{array}{l}\text { Saharan Revolutionary Armed Front } \\
\text { (FARS) }\end{array}$ & Nationalist & 1997 & 1997 & Niger & Armed Assault, Bombing/Explosion & Military, Private Citizens \& Property \\
\hline Saif-ul-Muslimeen & Religious & 2003 & - & Afghanistan & Bombing/Explosion & Military, NGO \\
\hline Sakar Group & Religious & 2010 & - & Indonesia & Unknown & $\begin{array}{l}\text { Businesses, Police, Religious } \\
\text { Figures/Institutions }\end{array}$ \\
\hline Salafia Jihadia & Religious & 1996 & - & Morocco & $\begin{array}{l}\text { Armed Assault, Bombing/Explosion, } \\
\text { Hostage Taking }\end{array}$ & $\begin{array}{l}\text { Businesses, Private Citizens \& Property, } \\
\text { Tourists }\end{array}$ \\
\hline $\begin{array}{l}\text { Salah al-Din Battalions/Salah al-Din } \\
\text { Brigades/Salah al-Din Squad }\end{array}$ & Nationalist & 2000 & - & Israel & Armed Assault, Bombing/Explosion & $\begin{array}{l}\text { Government (Diplomatic), Military, } \\
\text { Private Citizens \& Property }\end{array}$ \\
\hline Sami al-Ghul Brigades & Nationalist & 2006 & - & Israel, Palestinian State & Bombing/Explosion & $\begin{array}{l}\text { Government (General), Private Citizens } \\
\text { \& Property }\end{array}$ \\
\hline $\begin{array}{l}\text { Samyukta Janatantrik Terai Mukti } \\
\text { Morcha (SJTMM) }\end{array}$ & Nationalist & 2007 & - & Nepal & Armed Assault, Bombing/Explosion & $\begin{array}{l}\text { Businesses, Government (General), } \\
\text { Transportation }\end{array}$ \\
\hline Samyukta Jatiya Mukti Morcha (SJMM) & Nationalist & 2010 & - & Nepal & $\begin{array}{l}\text { Armed Assault, Bombing/Explosion, } \\
\text { Facility/Infrastructure Attack }\end{array}$ & $\begin{array}{l}\text { Businesses, Government (General), } \\
\text { Military, Private Citizens \& Property, } \\
\text { Telecommunication, Utilities, Violent } \\
\text { Political Party }\end{array}$ \\
\hline Sanathan Sanstha & $\begin{array}{l}\text { Right- } \\
\text { Wing }\end{array}$ & 2008 & - & India & Assassination, Bombing/Explosion & Private Citizens \& Property \\
\hline Sandval (Union) Movement/Sadval & Nationalist & 1994 & 1994 & Azerbaijan, Russia & Bombing/Explosion & Police, Private Citizens \& Property \\
\hline Sans Defaite & Nationalist & 1992 & - & Burundi & Armed Assault & $\begin{array}{l}\text { Government (General), Private Citizens } \\
\text { \& Property }\end{array}$ \\
\hline Sans Echec & Nationalist & 1995 & - & Burundi & Armed Assault & $\begin{array}{l}\text { Government (General), Private Citizens } \\
\text { \& Property }\end{array}$ \\
\hline Saraya al-Khorasani & Nationalist & 2013 & - & Iraq & Armed Assault, Bombing/Explosion & Terrorists/Non-State Militia \\
\hline Saraya al-Mukhtar (SaM) & Nationalist & 2013 & - & Bahrain, Saudi Arabia & Bombing/Explosion & Police \\
\hline Saraya al-Muqawama al-Sha'biya (SMS) & Religious & 2012 & - & Bahrain, Saudi Arabia & $\begin{array}{l}\text { Armed Assault, Bombing/Explosion, } \\
\text { Unarmed Assault }\end{array}$ & Government (General) \\
\hline Saraya al-Salam & Nationalist & 2014 & - & Iraq & Armed Assault, Bombing/Explosion & Terrorists/Non-State Militia \\
\hline $\begin{array}{l}\text { Saraya al-Shuhuada al-Jihadiyah fi al- } \\
\text { Iraq }\end{array}$ & Religious & 2004 & - & Iraq & Hostage Taking & Private Citizens \& Property \\
\hline $\begin{array}{l}\text { Saraya Defend Benghazi } \\
\text { (BDB)/Benghazi Defense Brigades }\end{array}$ & Religious & 2016 & - & Libya & Armed Assault, Bombing/Explosion & $\begin{array}{l}\text { Businesses, Military, Terrorists/Non- } \\
\text { State Militia, Utilities }\end{array}$ \\
\hline Saraya Jarabulus & Nationalist & 2014 & - & Syria & Armed Assault & Terrorists/Non-State Militia \\
\hline Saraya Usud al-Tawhid & Religious & 2004 & - & Iraq & Hostage Taking & Military \\
\hline
\end{tabular}




\begin{tabular}{|c|c|c|c|c|c|c|}
\hline VEO Name & Type & Emerged & Ended & Countries & Type of Attack & Target Type \\
\hline Sardinian Autonomy Movement (MAS) & Nationalist & 2002 & 2002 & Italy & Bombing/Explosion & $\begin{array}{l}\text { Government (General), Journalists \& } \\
\text { Media }\end{array}$ \\
\hline Sauderland Group & $\begin{array}{l}\text { Right- } \\
\text { Wing }\end{array}$ & 2006 & - & Germany & Bombing/Explosion & Military, Private Citizens \& Property \\
\hline Save Kashmir Movement & Nationalist & 2002 & - & India & Assassination, Bombing/Explosion & $\begin{array}{l}\text { Government (General), Private Citizens } \\
\text { \& Property }\end{array}$ \\
\hline Saved Sect & Religious & 2005 & - & United Kingdom & $\begin{array}{l}\text { Armed Assault, Bombing/Explosion, } \\
\text { Unarmed Assault }\end{array}$ & Businesses, Utilities \\
\hline $\begin{array}{l}\text { Secret Army for the Liberation of } \\
\text { Cabinda (ELSC) }\end{array}$ & Nationalist & 2003 & - & Angola & Armed Assault & Government (General) \\
\hline $\begin{array}{l}\text { Secret Organization to Safeguard the } \\
\text { Algerian Republic (SOSAR/OSSRA) }\end{array}$ & Nationalist & 1993 & - & Algeria, France & Armed Assault & Private Citizens \& Property \\
\hline Sect of Revolutionaries & Left-Wing & 2009 & - & Greece & $\begin{array}{l}\text { Armed Assault, Assassination, } \\
\text { Facility/Infrastructure Attack }\end{array}$ & Journalists \& Media, Police \\
\hline Seleka Coalition Central Africa Republic & Left-Wing & 2007 & - & The Central African Republic & $\begin{array}{l}\text { Armed Assault, Assassination, } \\
\text { Bombing/Explosion, Hostage Taking }\end{array}$ & $\begin{array}{l}\text { Government (Diplomatic), Government } \\
\text { (General), Military, NGO, Private } \\
\text { Citizens \& Property, Religious } \\
\text { Figures/Institutions }\end{array}$ \\
\hline $\begin{array}{l}\text { Self Defense Forces of Nagorno- } \\
\text { Karabakh/Nagorno-Karabakh Republic } \\
\text { Defense Army (SFNK) }\end{array}$ & Nationalist & 1992 & - & Armenia, Azerbaijan & Armed Assault & Terrorists/Non-State Militia \\
\hline Servants of Islam Organization & Religious & 1992 & 1992 & India & Armed Assault & Military \\
\hline Shabab al-Tawhid (ST) & Religious & 2014 & - & Libya, Tunisia & Hostage Taking & Government (General) \\
\hline Shabelle Valley State Militia (SVS) & Nationalist & 2011 & - & Ethiopia, Somalia & $\begin{array}{l}\text { Armed Assault, Assassination, } \\
\text { Bombing/Explosion }\end{array}$ & $\begin{array}{l}\text { Private Citizens \& Property, } \\
\text { Terrorists/Non-State Militia }\end{array}$ \\
\hline Shahin & Nationalist & 1992 & 1992 & Iran & Bombing/Explosion & Government (Diplomatic) \\
\hline $\begin{array}{l}\text { Shamiya Front/Levant Front/al-Jabha } \\
\text { al-Shamiya }\end{array}$ & Nationalist & 2014 & - & Syria & Armed Assault, Bombing/Explosion & $\begin{array}{l}\text { Government (General), Military, Private } \\
\text { Citizens \& Property, } \\
\text { Telecommunication, Terrorists/Non- } \\
\text { State Militia }\end{array}$ \\
\hline Shan State Army (SSA) & Nationalist & 2008 & - & Myanmar, Burma & Armed Assault, Bombing/Explosion & $\begin{array}{l}\text { Businesses, Military, Police, Private } \\
\text { Citizens \& Property, Religious } \\
\text { Figures/Institutions }\end{array}$ \\
\hline $\begin{array}{l}\text { Shan United Revolutionary Army } \\
\text { (SURA) }\end{array}$ & Nationalist & 2003 & - & Myanmar, Burma & Armed Assault, Bombing/Explosion & $\begin{array}{l}\text { Businesses, Police, Private Citizens \& } \\
\text { Property, Utilities }\end{array}$ \\
\hline Sheikh Abu al-Nur al-Maqdisi Brigade & Religious & 2014 & - & Palestinian State, Syria & Armed Assault & Terrorists/Non-State Militia \\
\hline Sheikh Omar Abdul Rahman Brigade & Religious & 2012 & - & Libya & $\begin{array}{l}\text { Armed Assault, Assassination, } \\
\text { Bombing/Explosion }\end{array}$ & Government (Diplomatic), NGO \\
\hline Sheikh Omar Hadid Brigade & Religious & 2015 & - & Israel & Bombing/Explosion & Private Citizens \& Property \\
\hline
\end{tabular}




\begin{tabular}{|c|c|c|c|c|c|c|}
\hline VEO Name & Type & Emerged & Ended & Countries & Type of Attack & Target Type \\
\hline Shield of Islam Brigade & Religious & 2010 & - & Iraq & Bombing/Explosion & Businesses, Military \\
\hline Shinwari Tribe & Nationalist & 1996 & - & Afghanistan, Pakistan & Bombing/Explosion & $\begin{array}{l}\text { Private Citizens \& Property, } \\
\text { Terrorists/Non-State Militia }\end{array}$ \\
\hline Shiromani Akali Dal (SAD (A)) & Nationalist & 1995 & - & India & Assassination & $\begin{array}{l}\text { Government (General), Private Citizens } \\
\text { \& Property }\end{array}$ \\
\hline Shiv Sena & Religious & 1992 & 1993 & India, Nepal & $\begin{array}{l}\text { Armed Assault, Facility/Infrastructure } \\
\text { Attack }\end{array}$ & $\begin{array}{l}\text { Government (General), Private Citizens } \\
\text { \& Property, Transportation }\end{array}$ \\
\hline Shora-e-Jihad & Nationalist & 1997 & 1999 & India, Pakistan & Unknown & Government (General) \\
\hline $\begin{array}{l}\text { Shura Council of Benghazi } \\
\text { Revolutionaries }\end{array}$ & Religious & 2014 & - & Libya & $\begin{array}{l}\text { Armed Assault, Bombing/Explosion, } \\
\text { Hostage Taking }\end{array}$ & $\begin{array}{l}\text { Military, Private Citizens \& Property, } \\
\text { Terrorists/Non-State Militia }\end{array}$ \\
\hline Shura Council of Mujahideen in Derna & Religious & 2014 & - & Libya & $\begin{array}{l}\text { Armed Assault, Bombing/Explosion, } \\
\text { Hostage Taking }\end{array}$ & $\begin{array}{l}\text { Government (General), Military, Private } \\
\text { Citizens \& Property, Religious } \\
\text { Figures/Institutions }\end{array}$ \\
\hline $\begin{array}{l}\text { Shura-e-Ittehadul Mujahedeen/Council } \\
\text { of United Holy Warriors }\end{array}$ & Religious & 2010 & - & Pakistan & Armed Assault & Military \\
\hline Shurafa al-Urdun & Religious & 2001 & 2002 & Jordan & Assassination & Businesses, Government (General) \\
\hline Sidama Liberation Front (SLF) & Nationalist & 1999 & - & Ethiopia & Unknown & Government (General) \\
\hline Sindhu Desh Liberation Army (SLA) & Nationalist & 2009 & - & Pakistan & Armed Assault, Bombing/Explosion & $\begin{array}{l}\text { Businesses, Educational Institutes, } \\
\text { Military, Private Citizens \& Property, } \\
\text { Religious Figures/Institutions, } \\
\text { Terrorists/Non-State Militia, } \\
\text { Transportation, Utilities }\end{array}$ \\
\hline Sipah-i-Mohammed (SMP) & Religious & 1993 & - & Pakistan & $\begin{array}{l}\text { Armed Assault, Assassination, } \\
\text { Bombing/Explosion }\end{array}$ & $\begin{array}{l}\text { Businesses, Journalists \& Media, Police, } \\
\text { Private Citizens \& Property, Religious } \\
\text { Figures/Institutions }\end{array}$ \\
\hline Sirri Powz & Religious & 2007 & - & Afghanistan & Armed Assault & Police \\
\hline Socialist Recovery Movement - Chile & Left-Wing & 1992 & 1992 & Chile & Bombing/Explosion & Government (General) \\
\hline Soldiers of the Caliphate - Kazakhstan & Religious & 2011 & - & Kazakhstan & Armed Assault, Bombing/Explosion & $\begin{array}{l}\text { Government (General), Private Citizens } \\
\text { \& Property }\end{array}$ \\
\hline Soldiers of the Caliphate in Egypt & Religious & 2014 & - & Egypt & Armed Assault, Bombing/Explosion & $\begin{array}{l}\text { Government (Diplomatic), Government } \\
\text { (General) }\end{array}$ \\
\hline $\begin{array}{l}\text { Soldiers of the Kingdom Assembly of } \\
\text { Iran/Tondar }\end{array}$ & Nationalist & 2008 & - & Iran & Assassination & Religious Figures/Institutions \\
\hline Soldiers of the Levant & Nationalist & 1999 & 2007 & Afghanistan, Syria & Armed Assault, Bombing/Explosion & $\begin{array}{l}\text { Educational Institutes, Government } \\
\text { (Diplomatic), Military }\end{array}$ \\
\hline Soldiers of the Prophet's Companions & Religious & 2005 & - & Iraq & Bombing/Explosion & Religious Figures/Institutions \\
\hline Solidarity for Political Prisoners & Left-Wing & 2002 & 2002 & Greece & Bombing/Explosion & Businesses \\
\hline
\end{tabular}




\begin{tabular}{|c|c|c|c|c|c|c|}
\hline VEO Name & Type & Emerged & Ended & Countries & Type of Attack & Target Type \\
\hline Solidarity Gas Canisters & Left-Wing & 2003 & 2003 & Greece & Bombing/Explosion & Businesses \\
\hline Solidarity with $17 \mathrm{~N}$ & Left-Wing & 2003 & 2003 & Greece & Bombing/Explosion & Private Citizens \& Property \\
\hline $\begin{array}{l}\text { Solidarity with Imprisoned Members of } \\
\text { Action Directe (AD) }\end{array}$ & Left-Wing & 2006 & 2007 & Greece & Bombing/Explosion & Businesses \\
\hline Somali Islamic Front & Religious & 2008 & - & Ethiopia, Somalia & $\begin{array}{l}\text { Armed Assault, Bombing/Explosion, } \\
\text { Hostage Taking }\end{array}$ & Government (General), NGO \\
\hline Somali National Alliance & Nationalist & 1992 & 1996 & Somalia & Armed Assault, Bombing/Explosion & $\begin{array}{l}\text { Government (Diplomatic), Government } \\
\text { (General), Military, NGO }\end{array}$ \\
\hline $\begin{array}{l}\text { Somali Reconciliation and Restoration } \\
\text { Council (SRRC) }\end{array}$ & $\begin{array}{l}\text { Right- } \\
\text { Wing }\end{array}$ & 2001 & - & Ethiopia, Somalia & Armed Assault & Military \\
\hline $\begin{array}{l}\text { South African National Defence Force } \\
\text { (SANDF) }\end{array}$ & Nationalist & 1994 & - & South Africa & Unknown & Unknown \\
\hline South Londonderry Volunteers (SLV) & Nationalist & 1998 & 2001 & Ireland, United Kingdom & Bombing/Explosion & $\begin{array}{l}\text { Private Citizens \& Property, Religious } \\
\text { Figures/Institutions }\end{array}$ \\
\hline South Maluku Republic (RMS) & Nationalist & 1998 & - & Indonesia, Netherlands & Bombing/Explosion & Government (General) \\
\hline $\begin{array}{l}\text { South Sudan Liberation } \\
\text { Movement/Army (SSLM/A) }\end{array}$ & Nationalist & 1999 & - & South Sudan & Armed Assault, Bombing/Explosion & $\begin{array}{l}\text { Businesses, Government (General), } \\
\text { Military, Transportation }\end{array}$ \\
\hline $\begin{array}{l}\text { South Sudan People Liberation } \\
\text { Movement in Opposition (SPLM/IO) }\end{array}$ & Nationalist & 2014 & - & South Sudan & $\begin{array}{l}\text { Armed Assault, Assassination, } \\
\text { Bombing/Explosion, } \\
\text { Facility/Infrastructure Attack, Hostage } \\
\text { Taking }\end{array}$ & $\begin{array}{l}\text { Businesses, Educational Institutes, } \\
\text { Government (Diplomatic), Government } \\
\text { (General), Military, Police, Private } \\
\text { Citizens \& Property, Religious } \\
\text { Figures/Institutions, Utilities }\end{array}$ \\
\hline Southern Front & Nationalist & 2014 & - & Syria & Armed Assault, Bombing/Explosion & $\begin{array}{l}\text { Businesses, Government (General), } \\
\text { Military, Private Citizens \& Property }\end{array}$ \\
\hline Southern Mobility Movement (SMM) & Nationalist & 2008 & - & Yemen & $\begin{array}{l}\text { Armed Assault, Assassination, } \\
\text { Bombing/Explosion, } \\
\text { Facility/Infrastructure Attack, Hostage } \\
\text { Taking }\end{array}$ & $\begin{array}{l}\text { Government (General), Military, Police, } \\
\text { Private Citizens \& Property, Utilities, } \\
\text { Violent Political Party }\end{array}$ \\
\hline Southern Resistance Brigades (SRB) & Nationalist & 2014 & - & Yemen & Armed Assault, Hostage Taking & Military \\
\hline $\begin{array}{l}\text { Special Purpose Islamic Regiment } \\
\text { (SIPR) }\end{array}$ & Nationalist & 1996 & 2002 & Georgia, Russia & $\begin{array}{l}\text { Armed Assault, Bombing/Explosion, } \\
\text { Hostage Taking }\end{array}$ & Businesses, Military \\
\hline $\begin{array}{l}\text { Squadrons of Terror/Dhamat Houmet } \\
\text { Daawa Salafia (DHDS) }\end{array}$ & Religious & 2000 & 2000 & Algeria & Armed Assault, Assassination & $\begin{array}{l}\text { Military, Police, Private Citizens \& } \\
\text { Property }\end{array}$ \\
\hline Sri Nakharo & Religious & 2001 & 2001 & Malaysia, Thailand & Armed Assault, Hostage Taking & Violent Political Party \\
\hline $\begin{array}{l}\text { Struggles for the Unity and Freedom of } \\
\text { Greater Syria }\end{array}$ & Nationalist & 2005 & 2005 & Lebanon & Assassination & Journalists \& Media \\
\hline
\end{tabular}




\begin{tabular}{|c|c|c|c|c|c|c|}
\hline VEO Name & Type & Emerged & Ended & Countries & Type of Attack & Target Type \\
\hline $\begin{array}{l}\text { Sudan Federal Democratic Alliance } \\
\text { (SFDA) }\end{array}$ & Nationalist & 1994 & - & Sudan & Armed Assault & Government (General), Military \\
\hline $\begin{array}{l}\text { Sudan Liberation Movement/Army } \\
\text { (SLA) }\end{array}$ & Nationalist & 2003 & - & Chad, Eritrea, Sudan & $\begin{array}{l}\text { Armed Assault, Bombing/Explosion, } \\
\text { Facility/Infrastructure Attack, } \\
\text { Hijacking, Hostage Taking }\end{array}$ & $\begin{array}{l}\text { Airports \& Aircraft, Businesses, } \\
\text { Educational Institutes, Government } \\
\text { (Diplomatic), Government (General), } \\
\text { Journalists \& Media, Maritime, Military, } \\
\text { NGO, Private Citizens \& Property }\end{array}$ \\
\hline Suf al-Haq Islamiya & Religious & 2006 & - & Israel, Palestinian State & Armed Assault, Bombing/Explosion & Businesses, Private Citizens \& Property \\
\hline $\begin{array}{l}\text { Sunni Movement for Indonesian } \\
\text { Society/Haraqah Sunni for Indonesian } \\
\text { Society (HASMI) }\end{array}$ & Religious & 2009 & - & Indonesia & Bombing/Explosion & $\begin{array}{l}\text { Government (Diplomatic), Private } \\
\text { Citizens \& Property, Religious } \\
\text { Figures/Institutions }\end{array}$ \\
\hline $\begin{array}{l}\text { Sunni Tehreek (ST)/Pakistani Sunni } \\
\text { Tehreek }\end{array}$ & Religious & 1992 & - & Pakistan & Armed Assault & $\begin{array}{l}\text { Government (General), Religious } \\
\text { Figures/Institutions, Terrorists/Non- } \\
\text { State Militia, Violent Political Party }\end{array}$ \\
\hline Supporters of Horst Ludwig Meyer & Left-Wing & 1999 & 1999 & Denmark & Bombing/Explosion & $\begin{array}{l}\text { Businesses, Government (Diplomatic), } \\
\text { Military }\end{array}$ \\
\hline $\begin{array}{l}\text { Supporters of Ocalan-The Hawks of } \\
\text { Thrace }\end{array}$ & Left-Wing & 1999 & 1999 & Greece & Bombing/Explosion & Government (Diplomatic) \\
\hline $\begin{array}{l}\text { Supporters of the Islamic League/Ansar } \\
\text { al-'Usba al-Islamiya }\end{array}$ & Nationalist & 1995 & - & Lebanon & Assassination & Government (General) \\
\hline $\begin{array}{l}\text { Supporters of the Khilafah/Supporters } \\
\text { of the Caliphate }\end{array}$ & Nationalist & 2012 & - & Syria & Armed Assault & Government (General), Military \\
\hline $\begin{array}{l}\text { Supreme Command for Jihad and } \\
\text { Liberation (SCJL) }\end{array}$ & Nationalist & 2007 & - & Iraq & $\begin{array}{l}\text { Armed Assault, Assassination, } \\
\text { Bombing/Explosion }\end{array}$ & Government (General), Military \\
\hline Supreme Military Council (SMC) & Nationalist & 2012 & - & Syria & Armed Assault & Government (General), Military \\
\hline Suqour al-Sham Brigade & Religious & 2011 & - & Belgium, Syria & Armed Assault, Bombing/Explosion & Government (General), Military \\
\hline Suqur al-Izz & Religious & 2013 & - & Syria & Armed Assault & Government (General) \\
\hline Suriname Liberation Front & Nationalist & 1994 & 1994 & Suriname & Hostage Taking & Utilities \\
\hline Swatantra Nepal Dal/Free Nepal Party & Left-Wing & 2010 & - & Nepal & Bombing/Explosion & Businesses \\
\hline Swaziland Youth Congress & Nationalist & 2003 & 2003 & Swaziland & Bombing/Explosion & Police \\
\hline Sword of Islam & Nationalist & 1998 & 2001 & Russia & Bombing/Explosion & Government (General) \\
\hline Swords of Righteousness Brigades & Religious & 2005 & - & Iraq & Armed Assault, Hostage Taking & Private Citizens \& Property \\
\hline Sympathizers of al-Qaida Organization & Religious & 2002 & 2002 & Yemen & $\begin{array}{l}\text { Armed Assault, Bombing/Explosion, } \\
\text { Facility/Infrastructure Attack }\end{array}$ & $\begin{array}{l}\text { Businesses, Government (Diplomatic), } \\
\text { Government (General), Utilities }\end{array}$ \\
\hline Syrian Islamic Front (SIF) & Religious & 2012 & - & Syria & Armed Assault, Bombing/Explosion & Government (General), Military \\
\hline $\begin{array}{l}\text { Syrian Islamic Liberation Front } \\
\text { (SLF/SILF) }\end{array}$ & Religious & 2012 & - & Syria & Armed Assault, Bombing/Explosion & Military \\
\hline
\end{tabular}




\begin{tabular}{|c|c|c|c|c|c|c|}
\hline VEO Name & Type & Emerged & Ended & Countries & Type of Attack & Target Type \\
\hline $\begin{array}{l}\text { Syrian Kurdish Democratic Union Party } \\
\text { (PYD) }\end{array}$ & $\overline{\text { Nationalist }}$ & 2003 & & Syria & Armed Assault & $\begin{array}{l}\text { Government (General), Military, } \\
\text { Terrorists/Non-State Militia }\end{array}$ \\
\hline Syrian Resistance & Left-Wing & 2013 & - & Syria, Turkey & Armed Assault & $\begin{array}{l}\text { Terrorists/Non-State Militia, Violent } \\
\text { Political Party }\end{array}$ \\
\hline Syrian Revolutionary Front & Nationalist & 2013 & - & Iraq, Syria & $\begin{array}{l}\text { Armed Assault, Assassination, } \\
\text { Bombing/Explosion }\end{array}$ & Military, Terrorists/Non-State Militia \\
\hline $\begin{array}{l}\text { Ta'ang National Liberation Army } \\
\text { (TNLA) }\end{array}$ & Nationalist & 1992 & - & Myanmar, Burma & $\begin{array}{l}\text { Armed Assault, Bombing/Explosion, } \\
\text { Hostage Taking }\end{array}$ & $\begin{array}{l}\text { Military, Police, Private Citizens \& } \\
\text { Property, Terrorists/Non-State Militia }\end{array}$ \\
\hline Tajamo Ansar al-Islam & Nationalist & 2012 & - & Syria & Bombing/Explosion & Government (General), Military \\
\hline Takfiri-Salafi Group & Religious & 2012 & - & Jordan, Syria & Armed Assault & Military \\
\hline Taliban & Religious & 1994 & - & Afghanistan, Pakistan & $\begin{array}{l}\text { Armed Assault, Assassination, } \\
\text { Bombing/Explosion, } \\
\text { Facility/Infrastructure Attack, } \\
\text { Hijacking, Hostage Taking, Unarmed } \\
\text { Assault }\end{array}$ & $\begin{array}{l}\text { Airports \& Aircraft, Businesses, } \\
\text { Educational Institutes, Food or Water } \\
\text { Supply, Government (Diplomatic), } \\
\text { Government (General), Journalists \& } \\
\text { Media, Military, NGO, Police, Private } \\
\text { Citizens \& Property, Religious } \\
\text { Figures/Institutions, } \\
\text { Telecommunication, Terrorists/Non- } \\
\text { State Militia, Tourists, Transportation, } \\
\text { Utilities, Violent Political Party }\end{array}$ \\
\hline Tamil Liberation Army & Nationalist & 1997 & 1997 & India, Sri Lanka & Bombing/Explosion & $\begin{array}{l}\text { Government (Diplomatic), Government } \\
\text { (General) }\end{array}$ \\
\hline $\begin{array}{l}\text { Tamil Makkal Viduthalai Pulikal } \\
\text { (TMVP) }\end{array}$ & Nationalist & 2004 & - & Sri Lanka & $\begin{array}{l}\text { Armed Assault, Assassination, } \\
\text { Bombing/Explosion, } \\
\text { Facility/Infrastructure Attack }\end{array}$ & $\begin{array}{l}\text { Government (General), Private Citizens } \\
\text { \& Property, Religious } \\
\text { Figures/Institutions, Terrorists/Non- } \\
\text { State Militia, Violent Political Party }\end{array}$ \\
\hline Tanzanian al-Shabaab & Religious & 2006 & - & Somalia, Tanzania & Hostage Taking & $\begin{array}{l}\text { Government (General), Military, } \\
\text { Tourists }\end{array}$ \\
\hline Tanzeem-ul-Islami-ul-Furqan (TIF) & $\begin{array}{l}\text { Right- } \\
\text { Wing }\end{array}$ & 2014 & - & Pakistan & $\begin{array}{l}\text { Armed Assault, Facility/Infrastructure } \\
\text { Attack, Unarmed Assault }\end{array}$ & Educational Institutes \\
\hline Tanzim & Nationalist & 1993 & 2005 & Israel & Bombing/Explosion, Hostage Taking & Private Citizens \& Property, Tourists \\
\hline Taong Bayan at Kawal & Left-Wing & 2006 & - & Philippines & Bombing/Explosion & Government (General), Military \\
\hline Tawhid and Jihad & Religious & 1999 & - & Iraq, Palestinian State & $\begin{array}{l}\text { Armed Assault, Bombing/Explosion, } \\
\text { Facility/Infrastructure Attack, Hostage } \\
\text { Taking }\end{array}$ & $\begin{array}{l}\text { Businesses, Government (Diplomatic), } \\
\text { Government (General), Maritime, } \\
\text { Military, Police, Private Citizens \& } \\
\text { Property, Religious Figures/Institutions, } \\
\text { Utilities }\end{array}$ \\
\hline
\end{tabular}




\begin{tabular}{|c|c|c|c|c|c|c|}
\hline $\begin{array}{l}\text { VEO Name } \\
\end{array}$ & Type & Emerged & Ended & Countries & Type of Attack & Target Type \\
\hline $\begin{array}{l}\text { Tawhid and Jihad Group in the Land of } \\
\text { the Philippines of Pride }\end{array}$ & Religious & 2012 & - & Philippines & Armed Assault & Military \\
\hline Tawhid Islamic Brigades & Religious & 2004 & 2004 & Egypt & Bombing/Explosion & Private Citizens \& Property \\
\hline Tehreek Nafaz-e-Aman & Nationalist & 2012 & - & Pakistan & $\begin{array}{l}\text { Assassination, Bombing/Explosion, } \\
\text { Hostage Taking }\end{array}$ & $\begin{array}{l}\text { Government (General), Journalists \& } \\
\text { Media, Private Citizens \& Property }\end{array}$ \\
\hline Tehreek-e-Galba-Islam (TEGI) & Religious & 2009 & - & Pakistan & Armed Assault & Police \\
\hline $\begin{array}{l}\text { Tehreek-e-Nafaz-e-Shariat-e- } \\
\text { Mohammadi (TNSM) }\end{array}$ & Religious & 1992 & - & Afghanistan, Pakistan & $\begin{array}{l}\text { Armed Assault, Bombing/Explosion, } \\
\text { Facility/Infrastructure Attack }\end{array}$ & $\begin{array}{l}\text { Businesses, Educational Institutes, } \\
\text { Military, Police, Private Citizens \& } \\
\text { Property, Religious Figures/Institutions, } \\
\text { Terrorists/Non-State Militia }\end{array}$ \\
\hline $\begin{array}{l}\text { Tehreek-e-Taliban Islami Pakistan } \\
\text { (TTIP/TTP) }\end{array}$ & Religious & 2014 & & Afghanistan, Pakistan & $\begin{array}{l}\text { Armed Assault, Arson, Assassination, } \\
\text { Bombing/Explosion, } \\
\text { Facility/Infrastructure Attack, Hostage } \\
\text { Taking }\end{array}$ & $\begin{array}{l}\text { Airports \& Aircraft, Businesses, } \\
\text { Educational Institutes, Food or Water } \\
\text { Supply, Government (Diplomatic), } \\
\text { Government (General), Journalists \& } \\
\text { Media, Military, NGO, Police, Private } \\
\text { Citizens \& Property, Religious } \\
\text { Figures/Institutions, } \\
\text { Telecommunication, Terrorists/Non- } \\
\text { State Militia, Tourists, Transportation, } \\
\text { Utilities, Violent Political Party }\end{array}$ \\
\hline $\begin{array}{l}\text { Tehreek-e-Taliban Pakistan Mehsud } \\
\text { Group }\end{array}$ & Religious & 2014 & - & Pakistan & Armed Assault & Terrorists/Non-State Militia \\
\hline Tehrik-e-Jihad (TEJ) & Nationalist & 1997 & - & India, Pakistan & Unknown & Unknown \\
\hline Tehrik-i-Taliban-Punjab & Religious & 2009 & - & India, Pakistan & Assassination, Bombing/Explosion & $\begin{array}{l}\text { Government (General), Military, Private } \\
\text { Citizens \& Property, Religious } \\
\text { Figures/Institutions }\end{array}$ \\
\hline Terai Army & Nationalist & 2008 & & Nepal & Bombing/Explosion, Hijacking & $\begin{array}{l}\text { Businesses, Government (General), } \\
\text { Military, Private Citizens \& Property, } \\
\text { Transportation }\end{array}$ \\
\hline Terai Cobra & Nationalist & 2008 & - & Nepal & Bombing/Explosion & $\begin{array}{l}\text { Government (General), NGO, Private } \\
\text { Citizens \& Property }\end{array}$ \\
\hline Terai Rastriya Mukti Sena (TRMS) & Nationalist & 2008 & - & Nepal & Bombing/Explosion, Hostage Taking & $\begin{array}{l}\text { Government (General), Journalists \& } \\
\text { Media, Private Citizens \& Property, } \\
\text { Transportation }\end{array}$ \\
\hline Territorial Anti-Imperialist Nuclei & Left-Wing & 1995 & - & Italy & Assassination, Bombing/Explosion & Government (General), Military \\
\hline The 29th Division & Nationalist & 2002 & - & Russia & Bombing/Explosion, Hostage Taking & NGO, Private Citizens \& Property \\
\hline
\end{tabular}




\begin{tabular}{|c|c|c|c|c|c|c|}
\hline VEO Name & Type & Emerged & Ended & Countries & Type of Attack & Target Type \\
\hline The Immortals & $\begin{array}{l}\text { Right- } \\
\text { Wing }\end{array}$ & 2009 & - & Germany & Unknown & Private Citizens \& Property \\
\hline $\begin{array}{l}\text { The Movement of the Patient Ones for } \\
\text { the Liberation of Palestine (HESN) }\end{array}$ & Religious & 2014 & - & Palestinian State & Unknown & Police \\
\hline Thus Far and No Further & Left-Wing & 2008 & - & Greece & Bombing/Explosion & Government (General) \\
\hline Tigers & Nationalist & 1998 & 1998 & Swaziland & Bombing/Explosion & Government (General) \\
\hline $\begin{array}{l}\text { Tigray People's Democratic Movement } \\
\text { (PTDM) }\end{array}$ & Left-Wing & 2008 & - & Ethiopia & Unknown & Military \\
\hline Tiwa National Revolutionary Front & Nationalist & 1996 & - & Bangladesh, India & Unknown & Unknown \\
\hline Tora Bora Nizami Mahaz (TBNM) & Nationalist & 2007 & - & Afghanistan, Pakistan & Armed Assault & Government (General), Military \\
\hline Torrid Winter & Left-Wing & 2003 & 2003 & Greece & Bombing/Explosion & Private Citizens \& Property \\
\hline Totally Anti-War Group (ATAG) & Left-Wing & 2001 & 2001 & France & Bombing/Explosion & Government (General) \\
\hline Toulouse Terrorist Group & Religious & 2006 & - & Belgium, France, United Kingdom & Armed Assault & Private Citizens \& Property \\
\hline $\begin{array}{l}\text { Transitional National Government - } \\
\text { Somalia (TNG) }\end{array}$ & Nationalist & 2000 & 2004 & Djibouti, Eritrea, Libya, Somalia & Armed Assault & Violent Political Party \\
\hline $\begin{array}{l}\text { Transnational Government of Tamil } \\
\text { Eelam (TGTE) }\end{array}$ & Nationalist & 2009 & - & $\begin{array}{l}\text { Australia, Canada, Denmark, Finland, } \\
\text { France, Germany, Ireland, Italy, New } \\
\text { Zealand, Norway, South Africa, Sudan, } \\
\text { Sweden, Switzerland, United Kingdom, } \\
\text { United States }\end{array}$ & Unknown & Government (General) \\
\hline Tripoli Brigades & Nationalist & 2011 & - & Ireland, Lebanon & Armed Assault & Private Citizens \& Property \\
\hline Tritiya Prastuti Committee & Left-Wing & 2002 & - & India & $\begin{array}{l}\text { Armed Assault, Facility/Infrastructure } \\
\text { Attack, Hostage Taking }\end{array}$ & $\begin{array}{l}\text { Businesses, Government (General), } \\
\text { Police, Private Citizens \& Property, } \\
\text { Terrorists/Non-State Militia, } \\
\text { Transportation }\end{array}$ \\
\hline Tuareg Brigade Mali & Nationalist & 2012 & - & Algeria, Libya & Hostage Taking & Military, NGO, Utilities \\
\hline Tulawie Clan & Nationalist & 1995 & 1995 & Philippines & Armed Assault & Military \\
\hline $\begin{array}{l}\text { Tupamaro Revolutionary Movement, } \\
\text { January } 23\end{array}$ & Left-Wing & 1998 & 2003 & Venezuela & Bombing/Explosion & $\begin{array}{l}\text { Educational Institutes, Government } \\
\text { (Diplomatic) }\end{array}$ \\
\hline $\begin{array}{l}\text { Turkish Kurdish United Revolutionary } \\
\text { Forces Platform (BDGP) }\end{array}$ & Nationalist & 1998 & - & Iraq, Turkey & Armed Assault, Bombing/Explosion & Government (General), Military \\
\hline $\begin{array}{l}\text { Turkish Revolutionary Communist } \\
\text { Union/Bolshevik (TIKB/B) }\end{array}$ & Left-Wing & 1998 & - & Germany, Turkey, United Kingdom & Bombing/Explosion & Police \\
\hline Turkistan Bhittani Group & Religious & 2007 & - & Pakistan & Armed Assault, Hostage Taking & $\begin{array}{l}\text { Terrorists/Non-State Militia, Violent } \\
\text { Political Party }\end{array}$ \\
\hline Turkistan Islamic Movement (TIM) & Nationalist & 1997 & - & Kyrgyzstan, Uzbekistan & $\begin{array}{l}\text { Armed Assault, Bombing/Explosion, } \\
\text { Hijacking }\end{array}$ & Military \\
\hline
\end{tabular}




\begin{tabular}{|c|c|c|c|c|c|c|}
\hline VEO Name & Type & Emerged & Ended & Countries & Type of Attack & Target Type \\
\hline $\begin{array}{l}\text { Turkistan Islamic Party (TIP)/East } \\
\text { Turkestan Islamic Movement (ETIM) }\end{array}$ & Religious & 2008 & - & $\begin{array}{l}\text { Afghanistan, China, Pakistan, Russia, } \\
\text { Syria }\end{array}$ & Armed Assault, Bombing/Explosion & $\begin{array}{l}\text { Private Citizens \& Property, } \\
\text { Transportation }\end{array}$ \\
\hline Turkmen's Brigade & Nationalist & 2013 & - & Syria & Armed Assault & Military \\
\hline Turks of Western Thrace & Nationalist & 1994 & 1994 & Greece & Bombing/Explosion & Government (General) \\
\hline Uamsho Group & Religious & 2001 & - & Tanzania & Armed Assault, Bombing/Explosion & $\begin{array}{l}\text { Government (General), Religious } \\
\text { Figures/Institutions }\end{array}$ \\
\hline Uighur Holy War Organization (UHWO) & Nationalist & 1997 & - & China & Assassination, Bombing/Explosion & Government (General), Police \\
\hline $\begin{array}{l}\text { Uighur Liberation Organization/East } \\
\text { Turkestan Liberation Organization } \\
\text { (ETLO) }\end{array}$ & Nationalist & 2000 & 2003 & China, Kazakhstan, Kyrgyzstan & $\begin{array}{l}\text { Armed Assault, Assassination, } \\
\text { Bombing/Explosion }\end{array}$ & $\begin{array}{l}\text { Businesses, Police, Private Citizens \& } \\
\text { Property }\end{array}$ \\
\hline Umar al-Mukhtar Martyr Forces & Nationalist & 2000 & 2000 & Palestinian State & Armed Assault, Bombing/Explosion & Military, Private Citizens \& Property \\
\hline Umbane People's Liberation Army & Nationalist & 2008 & - & Swaziland & Bombing/Explosion & Transportation, Utilities \\
\hline Ummah Tamir-e-Nau (UTN) & Religious & 2000 & - & Afghanistan, Pakistan & Hostage Taking & Unknown \\
\hline Uncontrolled Rage & Left-Wing & 2002 & 2002 & Greece & Bombing/Explosion & Businesses \\
\hline $\begin{array}{l}\text { Underground Government of the Free } \\
\text { Democratic People of Laos }\end{array}$ & Nationalist & 2000 & - & Lao, People's Democratic Republic & Bombing/Explosion & Businesses, Tourists, Transportation \\
\hline Unified Military Command of Chad & Nationalist & 2008 & - & Chad & Unknown & Government (General), Military \\
\hline Unified Unit of Jihad & Nationalist & 1993 & 1993 & Algeria & Unknown & Private Citizens \& Property \\
\hline Union of Congolese Patriots (UPC) & Nationalist & 2001 & - & Congo, Democratic Republic, Uganda & Armed Assault & $\begin{array}{l}\text { Airports \& Aircraft, NGO, Private } \\
\text { Citizens \& Property }\end{array}$ \\
\hline Union of Democratic Forces (UFD) & Nationalist & 1992 & - & Chad & Hostage Taking & Private Citizens \& Property \\
\hline $\begin{array}{l}\text { Union of Democratic Forces for Unity } \\
\text { (UFDR) }\end{array}$ & Nationalist & 2003 & - & Central African Republic, Chad, Sudan & Armed Assault & Terrorists/Non-State Militia \\
\hline $\begin{array}{l}\text { Union of Forces for Democracy and } \\
\text { Development (UFDD) }\end{array}$ & Nationalist & 2006 & - & Chad & Armed Assault & $\begin{array}{l}\text { Government (Diplomatic), Military, } \\
\text { Private Citizens \& Property }\end{array}$ \\
\hline $\begin{array}{l}\text { Union of Patriots for the Total } \\
\text { Liberation of Cote d'Ivoire (ULPTCI) }\end{array}$ & Nationalist & 2002 & & Cote D'Ivoire & Unknown & Terrorists/Non-State Militia \\
\hline Union of Peaceful Citizens of Algeria & Nationalist & 1993 & 1994 & Algeria, Spain & Hijacking & Airports \& Aircraft \\
\hline Union of Republican Forces (UFR) & Nationalist & 2003 & - & The Central African Republic & Armed Assault & Government (General) \\
\hline $\begin{array}{l}\text { Union of Revolutionary Communists in } \\
\text { Turkey (TIKB) }\end{array}$ & Left-Wing & 1999 & 1999 & Germany, Turkey, United Kingdom & Armed Assault, Bombing/Explosion & Government (General) \\
\hline Union of Volunteers Yerkrapah (UVY) & Nationalist & 1993 & - & Armenia, Azerbaijan & Armed Assault & $\begin{array}{l}\text { Private Citizens \& Property, Religious } \\
\text { Figures/Institutions }\end{array}$ \\
\hline United Achik National Front (UNAF) & Nationalist & 2004 & - & Bangladesh, India & $\begin{array}{l}\text { Armed Assault, Hostage Taking, } \\
\text { Unarmed Assault }\end{array}$ & Government (General) \\
\hline
\end{tabular}




\begin{tabular}{|c|c|c|c|c|c|c|}
\hline VEO Name & Type & Emerged & Ended & Countries & $\begin{array}{c}\text { Type of Attack } \\
\end{array}$ & $\begin{array}{c}\text { Target Type } \\
\end{array}$ \\
\hline United Action Council & Religious & 1994 & 1994 & Bangladesh & $\begin{array}{l}\text { Bombing/Explosion, } \\
\text { Facility/Infrastructure Attack, Unarmed } \\
\text { Assault }\end{array}$ & $\begin{array}{l}\text { Government (General), Police, Private } \\
\text { Citizens \& Property }\end{array}$ \\
\hline United Bengali Liberation Front (UBLF) & Nationalist & 1999 & & India & $\begin{array}{l}\text { Armed Assault, Bombing/Explosion, } \\
\text { Hostage Taking }\end{array}$ & $\begin{array}{l}\text { Businesses, Government (General), } \\
\text { Private Citizens \& Property, } \\
\text { Terrorists/Non-State Militia, } \\
\text { Transportation }\end{array}$ \\
\hline United Company of Holy War & Religious & 1993 & 1993 & Algeria & Assassination, Hostage Taking & Private Citizens \& Property \\
\hline $\begin{array}{l}\text { United Democratic Liberation Army } \\
\text { (UDLF/UDLA) }\end{array}$ & Nationalist & 2009 & - & India & Armed Assault, Hostage Taking & $\begin{array}{l}\text { Businesses, Police, Private Citizens \& } \\
\text { Property, Transportation }\end{array}$ \\
\hline $\begin{array}{l}\text { United Democratic Terai Liberation } \\
\text { Front (UDTLF) }\end{array}$ & Nationalist & 2008 & - & India, Nepal & Hostage Taking & Government (General) \\
\hline $\begin{array}{l}\text { United Front for Democracy Against } \\
\text { Dictatorship (UDD) }\end{array}$ & Nationalist & 2006 & - & Thailand & $\begin{array}{l}\text { Bombing/Explosion, } \\
\text { Facility/Infrastructure Attack }\end{array}$ & $\begin{array}{l}\text { Businesses, Government (General), } \\
\text { Journalists \& Media, Police, Private } \\
\text { Citizens \& Property, Religious } \\
\text { Figures/Institutions, Utilities }\end{array}$ \\
\hline $\begin{array}{l}\text { United Front for Democratic Change } \\
\text { (FUC) }\end{array}$ & Nationalist & 2006 & - & Chad & Armed Assault, Hostage Taking & $\begin{array}{l}\text { Government (General), NGO, Private } \\
\text { Citizens \& Property }\end{array}$ \\
\hline $\begin{array}{l}\text { United Front for Nigeria's Liberation } \\
\text { (UFNL) }\end{array}$ & Nationalist & 1996 & 1996 & Nigeria & Bombing/Explosion & Government (General) \\
\hline $\begin{array}{l}\text { United Kuki Liberation Army - Military } \\
\text { Council (UKLA-MC) }\end{array}$ & Nationalist & 2009 & - & India & Hostage Taking & Government (General) \\
\hline United Kuki Liberation Front (UKLF) & Nationalist & 1999 & - & India & $\begin{array}{l}\text { Armed Assault, Assassination, Hostage } \\
\text { Taking }\end{array}$ & $\begin{array}{l}\text { Government (General), Journalists \& } \\
\text { Media, Private Citizens \& Property }\end{array}$ \\
\hline $\begin{array}{l}\text { United Liberation Front of Barak Valley } \\
\text { (ULFBV) - Bangladesh }\end{array}$ & Nationalist & 2002 & - & Bangladesh & Armed Assault & $\begin{array}{l}\text { Businesses, Government (General), } \\
\text { Private Citizens \& Property }\end{array}$ \\
\hline $\begin{array}{l}\text { United Liberation Movement for } \\
\text { Democracy in Liberia - Alhaji G.V. } \\
\text { Kromah (ULIMO-K) }\end{array}$ & Nationalist & 1994 & 1997 & Liberia & Unknown & Private Citizens \& Property \\
\hline $\begin{array}{l}\text { United Liberation Movement for } \\
\text { Democracy in Liberia - Roosevelt } \\
\text { Johnson (ULIMO-J) }\end{array}$ & Nationalist & 1994 & 1997 & Liberia & Unknown & $\begin{array}{l}\text { Government (General), Private Citizens } \\
\text { \& Property }\end{array}$ \\
\hline $\begin{array}{l}\text { United National Liberation Front } \\
\text { (UNLF) }\end{array}$ & Nationalist & 2003 & - & India & Armed Assault, Bombing/Explosion & $\begin{array}{l}\text { Military, Police, Private Citizens \& } \\
\text { Property, Terrorists/Non-State Militia, } \\
\text { Transportation }\end{array}$ \\
\hline
\end{tabular}




\begin{tabular}{|c|c|c|c|c|c|c|}
\hline $\begin{array}{l}\text { VEO Name } \\
\end{array}$ & Type & Emerged & Ended & Countries & Type of Attack & Target Type \\
\hline $\begin{array}{l}\text { United People's Democratic Front } \\
\text { (UPDF) }\end{array}$ & Nationalist & 1998 & - & Bangladesh & $\begin{array}{l}\text { Armed Assault, Assassination, } \\
\text { Bombing/Explosion, Hostage Taking }\end{array}$ & $\begin{array}{l}\text { Businesses, Government (General), } \\
\text { Private Citizens \& Property, } \\
\text { Terrorists/Non-State Militia }\end{array}$ \\
\hline $\begin{array}{l}\text { United People's Democratic Solidarity } \\
\text { (UPDS) }\end{array}$ & Nationalist & 1999 & 2006 & India & $\begin{array}{l}\text { Armed Assault, Bombing/Explosion, } \\
\text { Hostage Taking }\end{array}$ & $\begin{array}{l}\text { Government (General), Private Citizens } \\
\text { \& Property, Transportation }\end{array}$ \\
\hline United Revolutionary Front & Left-Wing & 1997 & 1999 & Venezuela & Bombing/Explosion & $\begin{array}{l}\text { Businesses, Educational Institutes, } \\
\text { Government (General), Military, Private } \\
\text { Citizens \& Property }\end{array}$ \\
\hline $\begin{array}{l}\text { United Revolutionary Front of Bhutan } \\
\text { (URFB) }\end{array}$ & Left-Wing & 2008 & - & Bhutan & Armed Assault, Bombing/Explosion & Government (General), Utilities \\
\hline $\begin{array}{l}\text { United Self-Defense Forces of Colombia } \\
\text { (AUC) }\end{array}$ & $\begin{array}{l}\text { Right- } \\
\text { Wing }\end{array}$ & 1997 & 2006 & Colombia & $\begin{array}{l}\text { Armed Assault, Assassination, Hostage } \\
\text { Taking, Unarmed Assault }\end{array}$ & $\begin{array}{l}\text { Businesses, Government (General), } \\
\text { Journalists \& Media, Police, Private } \\
\text { Citizens \& Property }\end{array}$ \\
\hline United Self-Defense Forces of Venezuela & $\begin{array}{l}\text { Right- } \\
\text { Wing }\end{array}$ & 2002 & - & Venezuela & Unknown & Government (General) \\
\hline United Tajik Opposition & Left-Wing & 1994 & 2003 & Tajikistan & Assassination & Government (General) \\
\hline United Wa State Army & Nationalist & 1995 & - & Myanmar, Burma & Unknown & Government (General), Military \\
\hline Urhobo Revolutionary Army (URA) & Nationalist & 2003 & - & Niger, Nigeria & Bombing/Explosion & $\begin{array}{l}\text { Government (General), Private Citizens } \\
\text { \& Property, Utilities }\end{array}$ \\
\hline Usd Allah & Religious & 2004 & 2004 & Iraq & Hostage Taking & Government (Diplomatic) \\
\hline Ussud Al-Anbar & Religious & 2013 & - & Iraq, Syria & Bombing/Explosion & Private Citizens \& Property \\
\hline Uygur Holy War Organization & Nationalist & 2001 & 2001 & China & Armed Assault & $\begin{array}{l}\text { Businesses, Government (General), } \\
\text { Police, Violent Political Party }\end{array}$ \\
\hline Vanguard of Red Youth (AKM) & Left-Wing & 2003 & 2003 & Russia & Bombing/Explosion & Government (General), Transportation \\
\hline Vanguards of Conquest (VOC) & Religious & 1993 & 2001 & Egypt & Assassination, Bombing/Explosion & Government (General) \\
\hline Vanguards of the Caliphate & Religious & 2013 & - & Libya & Unknown & Religious Figures/Institutions \\
\hline $\begin{array}{l}\text { Variant Behavior for the Proliferation of } \\
\text { Revolutionary Terrorism }\end{array}$ & Left-Wing & 2012 & - & Greece & Bombing/Explosion & Businesses \\
\hline Vehabije & Religious & 2010 & - & Bosnia and Herzegovina & Bombing/Explosion & Government (Diplomatic) \\
\hline $\begin{array}{l}\text { Victorious Sect - Egypt/al-Taifa al- } \\
\text { Mansura }\end{array}$ & Religious & 2006 & - & Egypt & Bombing/Explosion & $\begin{array}{l}\text { Private Citizens \& Property, Religious } \\
\text { Figures/Institutions, Tourists, Utilities }\end{array}$ \\
\hline Vigorous Burmese Student Warriors & Left-Wing & 1999 & 2004 & Myanmar, Burma, Thailand & Bombing/Explosion, Hostage Taking & $\begin{array}{l}\text { Government (Diplomatic), Private } \\
\text { Citizens \& Property, Transportation }\end{array}$ \\
\hline Vigrid - Norway & $\begin{array}{l}\text { Right- } \\
\text { Wing }\end{array}$ & 1998 & - & Norway & Armed Assault, Unarmed Assault & $\begin{array}{l}\text { Private Citizens \& Property, Religious } \\
\text { Figures/Institutions }\end{array}$ \\
\hline Vitalunismo & Nationalist & 1999 & 2002 & France, Italy & Hijacking & Airports \& Aircraft \\
\hline Viviana Gallardo Command (VGC) & Nationalist & 1996 & 1996 & Costa Rica, Nicaragua & Hostage Taking & Government (Diplomatic) \\
\hline
\end{tabular}




\begin{tabular}{|c|c|c|c|c|c|c|}
\hline VEO Name & Type & Emerged & Ended & Countries & Type of Attack & Target Type \\
\hline Vojska Republike Srpske (VRS) & Nationalist & 1992 & 2006 & Bosnia and Herzegovina & Armed Assault & Unknown \\
\hline Vostok Battalion & Nationalist & 1999 & - & Russia, Ukraine & Armed Assault, Bombing/Explosion & Military, Terrorists/Non-State Militia \\
\hline Waffen SS & $\begin{array}{l}\text { Right- } \\
\text { Wing }\end{array}$ & 1998 & 1998 & Latvia & Bombing/Explosion & Religious Figures/Institutions \\
\hline Wahhabi Movement & Religious & 2010 & - & Bosnia and Herzegovina & Bombing/Explosion & Police \\
\hline Walla3 & Nationalist & 2014 & - & Egypt & Bombing/Explosion & Government (General) \\
\hline War that was Never Declared & Left-Wing & 2003 & 2003 & Greece & $\begin{array}{l}\text { Bombing/Explosion, } \\
\text { Facility/Infrastructure Attack }\end{array}$ & Businesses \\
\hline We Save Uganda Now & Nationalist & 2016 & - & Uganda & Armed Assault & $\begin{array}{l}\text { Government (General), Private Citizens } \\
\text { \& Property }\end{array}$ \\
\hline We Who Built Sweden & Left-Wing & $\begin{array}{l}1997 \\
\end{array}$ & $\begin{array}{l}1997 \\
\end{array}$ & Sweden & Bombing/Explosion & Private Citizens \& Property \\
\hline Weichan Auka Mapu & Nationalist & 2016 & - & Chile & $\begin{array}{l}\text { Armed Assault, Facility/Infrastructure } \\
\text { Attack }\end{array}$ & Police, Religious Figures/Institutions \\
\hline Werewolf Commando & Left-Wing & 2013 & - & Germany, Netherlands & Unknown & Government (General) \\
\hline West Indonesia Mujahideen & Religious & 2015 & - & Indonesia & Hostage Taking & Military \\
\hline West Nile Bank Front (WNBF) & Left-Wing & 1995 & 1998 & Congo, Democratic Republic, Uganda & Unknown & NGO \\
\hline Western European Bloodline & $\begin{array}{l}\text { Right- } \\
\text { Wing }\end{array}$ & 2012 & - & Canada & Armed Assault & Private Citizens \& Property \\
\hline White Army - South Sudan & Nationalist & 2011 & - & Sudan & Armed Assault & Private Citizens \& Property \\
\hline White Justice & $\begin{array}{l}\text { Right- } \\
\text { Wing }\end{array}$ & 2006 & - & Czech Republic & Armed Assault, Bombing/Explosion & Private Citizens \& Property \\
\hline White Legion & Nationalist & 1997 & 1998 & Georgia & Bombing/Explosion, Hostage Taking & $\begin{array}{l}\text { Government (Diplomatic), Police, } \\
\text { Private Citizens \& Property }\end{array}$ \\
\hline White Legion & $\begin{array}{l}\text { Right- } \\
\text { Wing }\end{array}$ & 2001 & 2003 & Ecuador & Armed Assault & Government (General) \\
\hline White Nationalist Party & Nationalist & 2002 & - & United Kingdom & Unknown & Private Citizens \& Property \\
\hline White Wolves & $\begin{array}{l}\text { Right- } \\
\text { Wing }\end{array}$ & 1999 & 1999 & United Kingdom & Bombing/Explosion & Businesses \\
\hline Wild Freedom & Left-Wing & 2013 & - & Greece & Bombing/Explosion & Businesses \\
\hline World Islamic Jihad Group & Religious & 1998 & 1998 & Yemen & Bombing/Explosion & $\begin{array}{l}\text { Government (General), } \\
\text { Telecommunication, Utilities }\end{array}$ \\
\hline World Uighur Youth Congress & Nationalist & 1996 & - & China, Germany & Unknown & Government (General) \\
\hline Yahya Ayyash Brigades & Religious & 2013 & - & Palestinian State & Bombing/Explosion & $\begin{array}{l}\text { Private Citizens \& Property, } \\
\text { Transportation }\end{array}$ \\
\hline Yakariay Bango Insurgent Group & Nationalist & 2012 & - & Thailand & Armed Assault, Bombing/Explosion & $\begin{array}{l}\text { Businesses, Military, Private Citizens \& } \\
\text { Property }\end{array}$ \\
\hline Yarmouk Martyrs Brigade & Nationalist & 2012 & - & Jordan, Syria & $\begin{array}{l}\text { Armed Assault, Assassination, } \\
\text { Bombing/Explosion, Hijacking, Hostage } \\
\text { Taking }\end{array}$ & $\begin{array}{l}\text { Government (Diplomatic), Military, } \\
\text { Terrorists/Non-State Militia }\end{array}$ \\
\hline
\end{tabular}




\begin{tabular}{|c|c|c|c|c|c|c|}
\hline VEO Name & Type & Emerged & Ended & Countries & Type of Attack & Target Type \\
\hline Yarmuk Jamaat & Religious & 2002 & - & Russia & $\begin{array}{l}\text { Armed Assault, Assassination, } \\
\text { Bombing/Explosion }\end{array}$ & Businesses, Police, Utilities \\
\hline Yemen Tribesman & Nationalist & 2011 & - & Yemen & Armed Assault & Military, Terrorists/Non-State Militia \\
\hline Yich Telga & Nationalist & 1992 & 1992 & France & Armed Assault & Journalists \& Media \\
\hline Yokoku Seiwa-Kai & $\begin{array}{l}\text { Right- } \\
\text { Wing }\end{array}$ & 1992 & 1992 & Japan & Assassination & Government (General) \\
\hline Young Communist League & Left-Wing & 2007 & - & Nepal & $\begin{array}{l}\text { Armed Assault, Bombing/Explosion, } \\
\text { Facility/Infrastructure Attack, Hostage } \\
\text { Taking }\end{array}$ & $\begin{array}{l}\text { Educational Institutes, Government } \\
\text { (General), Police, Private Citizens \& } \\
\text { Property }\end{array}$ \\
\hline Young Liberators of Pattani & Nationalist & 2002 & 2002 & Thailand & Facility/Infrastructure Attack & Educational Institutes \\
\hline $\begin{array}{l}\text { Young Officer Union of the New } \\
\text { Generation and Reformist Armed Forces } \\
\text { of the Philippines (YOU-RAFP) }\end{array}$ & Nationalist & 2006 & - & Philippines & Bombing/Explosion & Government (General) \\
\hline Young Patriots - Ivory Coast & Nationalist & 2006 & - & Cote D'Ivoire & Armed Assault & NGO \\
\hline Youth for Revolution - South Africa & Nationalist & 1992 & 1992 & South Africa & Facility/Infrastructure Attack & Government (General) \\
\hline Youth Movement for Change (YMC) & Nationalist & 2014 & - & Algeria, Morocco & Unknown & Terrorists/Non-State Militia \\
\hline Zahir Baibars Battalion & Religious & 2009 & - & Afghanistan, Kazakhstan, Pakistan & Bombing/Explosion & Government (General) \\
\hline Zarqawi Network & Religious & 1999 & - & Iraq, Jordan & Assassination, Bombing/Explosion & NGO, Private Citizens \& Property \\
\hline Zeliangrong United Front (ZUF) & Nationalist & 2011 & - & India & $\begin{array}{l}\text { Armed Assault, Bombing/Explosion, } \\
\text { Hostage Taking }\end{array}$ & $\begin{array}{l}\text { Government (General), Military, Police, } \\
\text { Private Citizens \& Property, } \\
\text { Terrorists/Non-State Militia }\end{array}$ \\
\hline Zelimkhan Akhmadov & Religious & 2002 & 2002 & Russia & Armed Assault & Military \\
\hline Zero Tolerance & Left-Wing & 2009 & - & Greece & $\begin{array}{l}\text { Bombing/Explosion, } \\
\text { Facility/Infrastructure Attack }\end{array}$ & Government (General) \\
\hline Zia-ur-Rehman Group & Religious & 2007 & 2008 & Afghanistan, Pakistan & $\begin{array}{l}\text { Armed Assault, Bombing/Explosion, } \\
\text { Hostage Taking }\end{array}$ & Military, Terrorists/Non-State Militia \\
\hline Zionist Resistance Fighters & Religious & 1999 & 1999 & Algeria & Armed Assault & Government (General) \\
\hline Zivinicke Ose & Religious & 1992 & 1995 & Bosnia and Herzegovina & Unknown & Unknown \\
\hline $\begin{array}{l}\text { Zomi Revolutionary Organization/Zomi } \\
\text { Revolutionary Army/Zomi } \\
\text { Revolutionary Volunteers }\end{array}$ & Nationalist & 1993 & - & India, Myanmar, Burma & Armed Assault & Educational Institutes, Military \\
\hline Zuwwar al-Imam Rida & Religious & 2013 & - & Lebanon, Syria & Hostage Taking & Airports \& Aircraft \\
\hline
\end{tabular}

